LUCAS MASSAROPPE

\title{
ESTIMAÇÃO DA CAUSALIDADE DE GRANGER NO CASO DE INTERAÇÃO NÃO-LINEAR
}


LUCAS MASSAROPPE

\section{ESTIMAÇÃO DA CAUSALIDADE DE GRANGER NO CASO DE INTERAÇÃO NÃO-LINEAR}

Tese apresentada à Escola Politécnica da Universidade de São Paulo para obtenção do título de Doutor em Ciências. 
LUCAS MASSAROPPE

\section{ESTIMAÇÃO DA CAUSALIDADE DE GRANGER NO CASO DE INTERAÇÃO NÃO-LINEAR}

Tese apresentada à Escola Politécnica da Universidade de São Paulo para obtenção do título de Doutor em Ciências.

Área de Concentração:

Sistemas Eletrônicos

Orientador: Prof. Dr. Luiz Antonio Baccalá 
Este exemplar foi revisado e alterado em relação à versão original, sob responsabilidade única do autor e com a anuência de seu orientador.

São Paulo, 05 de Outubro de 2016.

Assinatura do autor

Assinatura do orientador

Massaroppe, Lucas

Estimação da causalidade de Granger no caso de interação não-linear. / L. Massaroppe. - versão corr. — São Paulo, 2016.

$109 \mathrm{p}$.

Tese (Doutorado) - Escola Politécnica da Universidade de São Paulo. Departamento de Engenharia Telecomunicações e Controle, 2016.

1. Causalidade. 2. Inferência. 3. Modelos Não-Lineares (Análise de Séries Temporais). I. Universidade de São Paulo. Escola Politécnica. Departamento de Engenharia de Telecomunicações e Controle. II. t. 
Dedico este trabalho aos meus pais, Maria Amélia e José Antonio, pela paciência e compreensão, fazendo sempre o possível para que eu chegasse até aqui. Continuamente ensinaram-me a agir com gratidão, respeito e que o estudo é a maior riqueza que se pode oferecer e adquirir. 


\section{AGRADECIMENTOS}

Esta tese é resultado de um longo caminho trilhado desde o início da minha graduação, aqui na EPUSP. Considero que estar no empreendimento da busca pelo conhecimento se deve graças à minha mãe, excelente exemplo de professora.

Uma vez dentro da Universidade, devo meus agradecimentos à Escola Politécnica da Universidade de São Paulo, instituição a que sou muito grato por ter me acolhido nos últimos doze anos da minha vida acadêmica. Nesse lugar, tive oportunidade de frequentar diversas aulas e diferentes laboratórios e tenho muito orgulho de fazer parte da comunidade USPiana.

Tenho que agradecer ao Professor Luiz Antonio Baccalá, orientador deste trabalho, que conheci em 2006, em uma disciplina sobre Processos Estocásticos, na graduação e sempre esteve disposto em esclarecer minhas dúvidas, durante as muitas horas de conversas, fazendo-me crescer no aspecto científico.

Agradeço à Laura, pois, em mim, ela deposita credibilidade, confiança e por ser uma incentivadora na superação de meus limites. Através de suas pesquisas e experiencias acadêmicas, sempre me ensina a manter o espírito crítico e científico ativo, com ética e seriedade. Nossas discussões são sempre muito úteis para meu aperfeiçoamento científico.

Da mesma forma, aplico esses agradecimentos à Silvia, que sempre soube me ouvir, ensinando e orientando-me a realizar minhas tarefas com paciência. Em momentos difíceis acolheu-me, mas sempre concedendo estímulos para que eu tivesse uma visão mais completa do meu entorno e, portanto, conseguisse seguir com minha pesquisa até o fim.

Registro um agradecimento especial às minhas sobrinhas Helena e Cecília, que à data desta tese, têm, respectivamente, quatro e um anos de idade. O carinho e alegria delas sempre me incentivam a seguir em frente.

Agradeço à minha irmã Bianca e seu marido Bruno, assim como meu irmão Bruno e sua esposa Cristiane. Meu grande muito obrigado pelo seu apoio, carinho e imensa compreensão nessa minha jornada em perseguir a carreira científica e acadêmica.

Estendo meus agradecimentos aos Professores Piqueira (EPUSP) e Sameshima (FMUSP), pelas boas críticas e sugestões oferecidas ainda no exame de qualificação e que, certamente, elevaram o nível final da tese.

Agradeço, ainda, a todos os Professores do Laboratório de Comunicações e Sinais (LCS) da Escola Politécnica da USP (EPUSP), que de forma direta e/ou indireta participaram de toda a minha formação e aos colegas, também do LCS, Amanda, Diana, Flavio Caduda, Flávio Pavan, Gilson e Pedro, pela colaboração e discussão de idéias ao longo 
desses anos, tornando o cotidiano e o trabalho mais agradáveis.

Finalmente, agradeço à CAPES (Coordenação de Aperfeiçoamento de Pessoal de Nível Superior) pelo apoio financeiro total dado a este trabalho, através de Bolsa de Doutorado, do Programa de Cota Institucional (Programa de Demanda Social - PDS). 
"Bem sei que é assustador sair de si mesmo, mas tudo o que é novo assusta."

(A Hora da Estrela - Clarice Lispector) 


\section{RESUMO}

Esta tese examina o problema de detecção de conectividade entre séries temporais no sentido de Granger no caso em que a natureza não linear das interações não permite sua determinação por meio de modelos autorregressivos lineares vetoriais. Mostra-se que é possível realizar esta detecção com auxílio dos chamados métodos de Kernel, que se tornaram populares em aprendizado por máquina ('machine learning') já que tais métodos permitem definir formas generalizadas de teste de Granger, coerência parcial direcionada e função de transferência direcionada. Usando simulações, mostram-se alguns exemplos de detecção nos quais fica também evidente que resultados assintóticos deduzidos originalmente para estimadores lineares podem ser generalizados de modo análogo, mostrando-se válidos no presente contexto kernelizado.

Palavras-chave: Coerência Parcial Direcionada. Função de Transferência Direcionada. Teste de Causalidade de Granger. Métodos de Kernels. Inferência estatística. Estatística Assintótica. Modelos Não-Lineares. Análise de Séries Temporais. 


\begin{abstract}
This work examines the connectivity detection problem between time series in the Granger sense when the nonlinear nature of interactions determination is impossible via linear vector autoregressive models, but is, nonetheless, feasible with the aid of the so-called Kernel methods that are popular in machine learning. The kernelization approach allows defining generalised versions for Granger tests, partial directed coherence and directed transfer function, which the simulation of some examples shows that the asymptotic detection results originally deducted for linear estimators, can also be employed under kernelization if suitably adapted.
\end{abstract}

Keywords: Partial Directed Coherence. Directed Transfer Function. Granger Causality Test. Kernel Methods. Statistical Inference. Asymptotics Statistics. Nonlinear Models. Time Series Analysis. 


\section{LISTA DE ILUSTRAÇÕES}

Figura 1 - Mapeamento de características: a idéia básica dos métodos de kernel. O mapeamento $\phi$ transforma o domínio (chamado de espaço de entrada) em outro de maior dimensão, ou seja, no espaço de funções de características. Observa-se que esse mapeamento é capaz de linearizar os dados. $\boldsymbol{\Sigma}_{\mathbf{1}}$ e $\boldsymbol{\Sigma}_{\mathbf{2}}$ representam as estruturas de covariancias dos dados nesses dois espaços. . . . . . . . . . . . . . . . 6

Figura 2 - Padrão de conectividade do exemplo 1. . . . . . . . . . . . 37

Figura 3 - Padrão de alcançabilidade do exemplo 1. . . . . . . . . . . . 37

Figura 4 - Realização típica do processo VAR(1), descrito por (3.2). . . . . . . . . 38

Figura 5 - $i$ PDC para uma realização de (3.2), considerando um nível de significância de $\alpha=0,01$. Utilizando o critério de escolha de ordem de HQ,

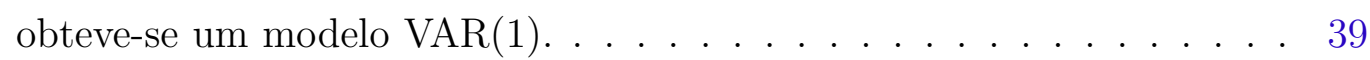

Figura 6 - $k n \mathrm{PDC}$ para uma realização de (3.2), utilizando o kernel $\kappa(\mathbf{x}, \mathbf{y})=$ $(1+\langle\mathbf{x}, \mathbf{y}\rangle)^{2}$, considerando um nível de significância de $\alpha=0,01$. Utilizando o critério de escolha de ordem de HQ, obteve-se um modelo $k \operatorname{VAR}(1) \ldots \ldots \ldots \ldots \ldots \ldots \ldots$

Figura 7 - $i$ DTF para uma realização de (3.2), considerando um nível de significância de $\alpha=0,01$. Utilizando o critério de escolha de ordem de HQ, obteve-se um modelo $\operatorname{VAR}(1) \ldots . . . . . . . .40$

Figura 8 - knDTF para uma realização de (3.2), utilizando o kernel $\kappa(\mathbf{x}, \mathbf{y})=$ $(1+\langle\mathbf{x}, \mathbf{y}\rangle)^{2}$, considerando um nível de significância de $\alpha=0,01$. Utilizando o critério de escolha de ordem de HQ, obteve-se um modelo $k \operatorname{VAR}(1) \ldots \ldots \ldots \ldots \ldots . \ldots \ldots$

Figura 9 - Padrão de conectividade e alcançabilidade do exemplo 2. . . . . . . . . 42

Figura 10 - Realização típica do processo descrito por (3.3), quando $c=1,0$ e $N=1024 \ldots \ldots \ldots \ldots \ldots \ldots \ldots$. . . . . . . . . . . . . . . . . . .

Figura 11 - knPDC para uma realização de (3.3), considerando um nível de significância de $\alpha=0,01$. Para a escolha da ordem do modelo, utilizou-se o

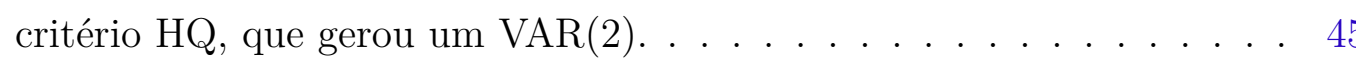

Figura 12 - knDTF para uma realização de (3.3), considerando um nível de significância de $\alpha=0,01$. Para a escolha da ordem do modelo, utilizou-se o

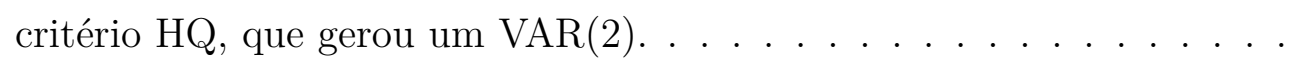

Figura 13 - knPDC para uma realização de (3.3), utilizando o kernel $\kappa(\mathbf{x}, \mathbf{y})=$ $\langle\mathbf{x}, \mathbf{y}\rangle^{2}$, considerando um nível de significância de $\alpha=0,01$. Para a escolha da ordem do modelo, utilizou-se o critério HQ, que gerou um $k \operatorname{VAR}(9) \ldots \ldots \ldots \ldots \ldots \ldots$ 
Figura 14 - knDTF para uma realização de (3.3), utilizando o kernel $\kappa(\mathbf{x}, \mathbf{y})=$ $\langle\mathbf{x}, \mathbf{y}\rangle^{2}$, considerando um nível de significância de $\alpha=0,01$. Para a escolha da ordem do modelo, utilizou-se o critério HQ, que gerou um $k \operatorname{VAR}(9) \ldots \ldots \ldots \ldots \ldots \ldots \ldots$

Figura 15 - Padrão de conectividade e alcançabilidade do exemplo 3. . . . . . . . . 48

Figura 16 - Realização típica do processo descrito por (3.5), quando $c=0,5$ e $N=1024 \ldots \ldots \ldots \ldots \ldots \ldots$

Figura 17 - Reconstrução dos atratores de $x(n)$ e $y(n)$, nas Figuras 17a e 17b, respectivamente e um gráfico de dispersão de $x(n)$ vs. $y(n)$, na Figura 17c. 51

Figura 18 - $i$ PDC para uma realização de (3.5), considerando um nível de significância de $\alpha=0,01$. Para a escolha da ordem do modelo, utilizou-se o critério HQ, que gerou um $\operatorname{VAR}(2) \ldots . . . . . .52$

Figura 19 - $i$ DTF para uma realização de (3.5), considerando um nível de significância de $\alpha=0,01$. Para a escolha da ordem do modelo, utilizou-se o

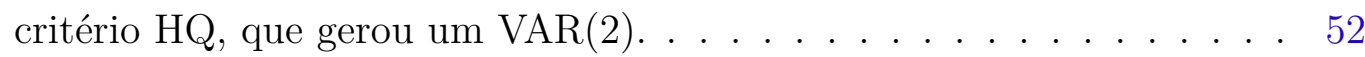

Figura 20 - knPDC para uma realização de (3.5), utilizando o kernel $\kappa(\mathbf{x}, \mathbf{y})=$ $\langle\mathbf{x}, \mathbf{y}\rangle^{4}$, considerando um nível de significância de $\alpha=0,01$. Para a escolha da ordem do modelo, utilizou-se o critério HQ, que gerou um $k \operatorname{VAR}(2)$.

Figura 21 - knDTF para uma realização de (3.5), utilizando o kernel $\kappa(\mathbf{x}, \mathbf{y})=$ $\langle\mathbf{x}, \mathbf{y}\rangle^{4}$, considerando um nível de significância de $\alpha=0,01$. Para a escolha da ordem do modelo, utilizou-se o critério HQ, que gerou um $k \operatorname{VAR}(2) \ldots \ldots \ldots \ldots \ldots \ldots \ldots$

Figura 22 - Padrão de conectividade e alcançabilidade do exemplo 4. . . . . . . . . 55

Figura 23 - Realização típica do processo descrito por (3.6), quando $N=1024$. . . 56

Figura 24 - $i$ PDC para uma realização de (3.6), considerando um nível de significância de $\alpha=0,01$. Para a escolha da ordem do modelo, utilizou-se o

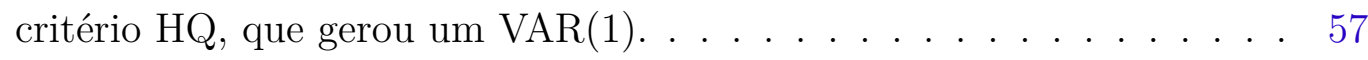

Figura 25 - $i$ DTF para uma realização de (3.6), considerando um nível de significância de $\alpha=0,01$. Para a escolha da ordem do modelo, utilizou-se o

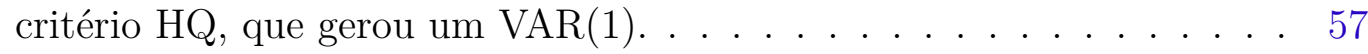

Figura 26 - knPDC para uma realização de (3.6), utilizando o kernel $\kappa(\mathbf{x}, \mathbf{y})=$ $\langle\mathbf{x}, \mathbf{y}\rangle^{2}$, considerando um nível de significância de $\alpha=0,01$. Para a escolha da ordem do modelo, utilizou-se o critério HQ, que gerou um $k \operatorname{VAR}(1) \ldots \ldots \ldots \ldots \ldots \ldots \ldots$

Figura 27 - knDTF para uma realização de (3.6), utilizando o kernel $\kappa(\mathbf{x}, \mathbf{y})=$ $\langle\mathbf{x}, \mathbf{y}\rangle^{2}$, considerando um nível de significância de $\alpha=0,01$. Para a escolha da ordem do modelo, utilizou-se o critério HQ, que gerou um $k \operatorname{VAR}(1) \ldots \ldots \ldots \ldots \ldots \ldots$ 
Figura 28 - Típico processo de filtragem. . . . . . . . . . . . . . 78

Figura 29 - Típico MOB de Hammerstein. . . . . . . . . . . . . . . . . . . . 81

Figura 30 - Típico MOB de Wiener. . . . . . . . . . . . . . . . . . . . 82

Figura 31 - Possível MOB de Wiener-Hammerstein. . . . . . . . . . . . . . . 82 


\section{LISTA DE TABELAS}

Tabela 1 - Taxas de verdadeiros positivos (VP) e falsos positivos (FP), para a GCT e $k n$ GCT, para o Exemplo 1. . . . . . . . . . . . . . . . 41

Tabela 2 - Taxas de verdadeiros positivos (VP) e falsos positivos (FP), para a PDC e $k n$ PDC, para o Exemplo 1. . . . . . . . . . . . . . . . . . . 41

Tabela 3 - Taxas de verdadeiros positivos (VP) e falsos positivos (FP), para a DTF e $k n$ DTF, para o Exemplo 1. . . . . . . . . . . . . . . . . 41

Tabela 4 - Tabela de contingência para o exemplo 2, referente ao $k n$ GCT. . . . . . 47

Tabela 5 - Tabela de contingência para o exemplo 2, referente ao $k n$ PDC. . . . . . 47

Tabela 6 - Tabela de contingência para o exemplo 2, referente ao $k n$ DTF. . . . . . 47

Tabela 7 - Tabela de contingência para o exemplo 3, referente ao $k n$ GCT. . . . . . 54

Tabela 8 - Tabela de contingência para o exemplo 3, referente ao $k n$ PDC. . . . . . 54

Tabela 9 - Tabela de contingência para o exemplo 3 , referente ao $k n$ DTF. . . . . . 54

Tabela 10 - Taxas de verdadeiros positivos (VP) e falsos positivos (FP), para o knGCT, para o Exemplo 4. . . . . . . . . . . . . . . . . 59

Tabela 11 - Taxas de verdadeiros positivos (VP) e falsos positivos (FP), para o knPDC, para o Exemplo 4. . . . . . . . . . . . . . 59

Tabela 12 - Taxas de verdadeiros positivos (VP) e falsos positivos (FP), para o knDTF, para o Exemplo 4. . . . . . . . . . . . . . . . 59 


\section{LISTA DE ABREVIATURAS E SIGLAS}

\begin{tabular}{|c|c|}
\hline EEG & Eletroencefalograma (Electroencephalogram) \\
\hline fMRI & $\begin{array}{l}\text { Ressonância magnética nuclear funcional } \\
\text { (Functional Magnetic Resonance Imaging) }\end{array}$ \\
\hline MEG & Magnetoencefalograma (Magnetoencephalogram) \\
\hline $\mathrm{CG}$ & Causalidade de Granger (Granger Causality) \\
\hline RKHS & Reproducing kernel Hilbert space \\
\hline $\operatorname{VAR}(p)$ & $\begin{array}{l}\text { Modelo vetorial autorregressivo de ordem } p \\
\text { (Vector Autoregressive Model of order } p \text { ) }\end{array}$ \\
\hline$k \operatorname{VAR}(p)$ & $\begin{array}{l}\text { Modelo vetorial autorregressivo kernelizado de ordem } p \\
\text { (kernelized Vector Autoregressive Model of order } p \text { ) }\end{array}$ \\
\hline VAR & $\begin{array}{l}\text { Processo vetorial autorregressivo } \\
\text { (Vector Autoregressive Process) }\end{array}$ \\
\hline$k \mathrm{VAR}$ & $\begin{array}{l}\text { Processo vetorial autorregressivo kernelizado } \\
\text { (kernelized Vector Autoregressive Process) }\end{array}$ \\
\hline$k n \mathrm{PDC}$ & $\begin{array}{l}\text { Coerência parcial direcionada-não-linear-kernelizada } \\
\text { (kernel-nonlinear-Partial directed coherence) }\end{array}$ \\
\hline $\mathrm{PDC}$ & Coerência parcial direcionada (Partial directed coherence) \\
\hline$k n \mathrm{DTF}$ & $\begin{array}{l}\text { Função de transferência direcionada-não-linear-kernelizada } \\
\text { (kernel-nonlinear-Directed Transfer Function) }\end{array}$ \\
\hline DTF & Função de transferência direcionada (Directed Transfer Function) \\
\hline$k n \mathrm{GCT}$ & $\begin{array}{l}\text { Teste da Causalidade de Granger-não-linear-kernelizada } \\
\text { (kernel-nonlinear-Granger Causality Test) }\end{array}$ \\
\hline GCT & Teste da Causalidade de Granger (Granger Causality Test) \\
\hline${ }_{k} \mathrm{FPE}$ & $\begin{array}{l}\text { Critério da predição final do erro kernelizado (Kernelized Final Predic- } \\
\text { tion Error) }\end{array}$ \\
\hline${ }_{k} \mathrm{AIC}$ & $\begin{array}{l}\text { Critério de informação de Akaike kernelizado (Kernelized Akaike Infor- } \\
\text { mation Criterion) }\end{array}$ \\
\hline
\end{tabular}


${ }_{k} \mathrm{HQ} \quad$ Critério de Hannan-Quinn kernelizado (Kernelized Hannan-Quinn Criterion)

${ }_{k}$ BIC Critério (Bayesiano) de Schwarz kernelizado (Kernelized Schwarz (Bayesian) Criterion)

RR Regressão ridge (Ridge Regression)

KRR Regressão ridge kernelizada (Kernel Ridge Regression)

knMVLS Mínimos quadrados multivariados kernelizados

(kernel-nonlinear-Multivariate Least Squares)

pdf Função densidade de probabilidade (Probability density function)

KDE Estimação de densidade via kernel (Kernel density estimation)

MOB Modelos Orientados à Blocos

NARMAX Modelo Não-Linear Autorregressivo de Média Móvel com Entrada Exógena

(Nonlinear Autoregressive Moving Average with Exogenous Inputs Model)

NARX Modelo Não-Linear Autorregressivo Exógena

(Nonlinear Autoregressive with Exogenous Inputs Model)

v.a. Variável aleatória

i.i.d. Independente identicamente distribuído

WN Ruído branco (White Noise) 


\section{LISTA DE SÍMBOLOS}

\begin{tabular}{|c|c|}
\hline$a$ & Escalar (minúscula) \\
\hline$a^{*}$ & Complexo conjugado de um escalar \\
\hline a & Vetor coluna (minúscula e em negrito) \\
\hline $\mathbf{a}(i)$ & Instância de vetor coluna no instante de tempo $i$ \\
\hline$a_{i}$ & $i$-ésima componente do vetor $\mathbf{a}$ \\
\hline A & Matriz (maiúscula e em negrito) \\
\hline$A_{i j}(\cdot)$ & Elemento $(i, j)$ da matriz $\mathbf{A}$ \\
\hline $\mathbf{a}_{i}(\cdot)$ & Linha $i$ ou coluna $i$ da matriz $\mathbf{A}$ (dependendo do contexto) \\
\hline $\mathbf{A}^{\top}$ & Matriz transposta \\
\hline $\mathbf{A}^{\mathrm{H}}$ & Matriz hermitiana \\
\hline $\mathbf{A}^{\dagger}$ & Pseudo-inversa de Penrose-Moore \\
\hline $\mathbf{A}^{-1}$ & Matriz inversa \\
\hline$\|\mathbf{a}\|_{p}$ & Norma $l_{p}$ de vetor \\
\hline$\widehat{\theta}$ & Estimativa da estatística $\theta$ \\
\hline$\lambda$ & Parâmetro de regularização da regressão ridge (kernelizada) \\
\hline $\mathbb{R}$ & Conjunto dos números reais \\
\hline $\mathbb{N}$ & Conjunto dos números naturais \\
\hline $\mathbb{Z}$ & Conjunto dos números inteiros \\
\hline$L^{2}(\mathbb{X})$ & Espaço das funções quadrado-integráveis \\
\hline$L^{\infty}(\mathbb{X})$ & Espaço das funções absolutamente-integráveis \\
\hline$\langle\mathbf{x}, \mathbf{y}\rangle$ & Produto interno entre $\mathbf{x}$ e $\mathbf{y}$ \\
\hline$f(\mathbf{x})$ & Vetor $\mathbf{x}$ avaliado por uma função $f(\cdot)$ \\
\hline $\mathbf{f}(\mathbf{x})$ & Vetor $\mathbf{x}$ avaliado por um vetor de funções $\mathbf{f}(\cdot)$ \\
\hline $\mathcal{F}[\cdot]$ & Operador transformada de Fourier \\
\hline
\end{tabular}




\begin{tabular}{|c|c|}
\hline $\log (\cdot)$ & Função logaritmo \\
\hline $\min _{\{\cdot\}}\{\cdot\}$ & Operador mínimo \\
\hline $\lim _{\{\cdot\}}\{\cdot\}$ & Operador limite \\
\hline & Coeficiente binomial \\
\hline$\phi(\mathbf{x})$ & Transformação de $\mathbf{x}$ para um espaço característico \\
\hline$\kappa(\cdot, \cdot)$ & Função de kernel \\
\hline $\mathbf{K}(\cdot, \cdot)$ & Matriz de kernel \\
\hline$\alpha_{i}$ & Coeficientes de expansão \\
\hline $\boldsymbol{\alpha}_{i}$ & Vetor de coeficientes de expansão \\
\hline$\alpha$ & Vetor solução do problema de mínimos quadrados \\
\hline$N$ & Número de pontos de uma série \\
\hline$D$ & Número de séries simultâneas, também chamados de canais \\
\hline $\mathbf{S}(\cdot)$ & Matriz densidade espectral \\
\hline $\mathbf{I}_{p}$ & Matriz identidade de dimensão $(p \times p)$ \\
\hline I & Matriz identidade de dimensão apropriada (dada pelo contexto) \\
\hline $\mathbf{0}_{p \times p}$ & Matriz nula de dimensão $(p \times p)$ \\
\hline 0 & Matriz nula de dimensão apropriada (dada pelo contexto) \\
\hline $\mathrm{N}\left(\mu, \sigma^{2}\right)$ & Distribuição normal de média $\mu$ e variância $\sigma^{2}$ \\
\hline $\mathrm{N}_{p}(\boldsymbol{\mu}, \Sigma)$ & $\begin{array}{l}\text { Distribuição normal multivariada, de dimensão }(p \times 1) \text { (ou } p \text {-variada), } \\
\text { com vetor de médias } \boldsymbol{\mu} \text { e estrutura de covariância } \boldsymbol{\Sigma}\end{array}$ \\
\hline$\chi_{\nu}^{2}$ & Distribuição (univariada) Chi-Quadrado, com $\nu$ graus de liberdade \\
\hline i.i.d.WN $\left(\mu, \sigma^{2}\right)$ & $\begin{array}{l}\text { Processo independente e identicamente distribuído, com estatística } \\
\text { de ruído branco }(\mathrm{WN}) \text { de média } \mu \text { e variância } \sigma^{2}\end{array}$ \\
\hline i.i.d. $\left(\mu, \sigma^{2}\right)$ & $\begin{array}{l}\text { Variável aleatória independente e identicamente distribuída de média } \\
\mu \text { e variância } \sigma^{2}\end{array}$ \\
\hline
\end{tabular}


i.i.d.p $(\boldsymbol{\mu}, \boldsymbol{\Sigma})$ Vetor de variáveis aleatórias, de dimensão $(p \times 1)$ (ou $p$-variada), independentes e identicamente distribuídas, com vetor de médias $\boldsymbol{\mu}$ e estrutura de covariância $\Sigma$

$\mathcal{H}_{0}, \mathcal{H}_{1} \quad$ Hipótese nula e alternativa, respectivamente

$\alpha \quad$ Nível de confiança

$\stackrel{d}{\rightarrow} \quad$ Convergência em distribuição

$\operatorname{plim}(\cdot) \quad$ Operador limite em probabilidade

$\mathbb{E}[\cdot] \quad$ Operador esperança matemática

$\operatorname{Var}[\cdot] \quad$ Operador variância matemática

$\operatorname{Cov}(\cdot) \quad$ Operador covariância matemática

$\mathbb{P}(\cdot) \quad$ Operador probabilidade

${ }_{\kappa} \lambda_{W} \quad$ Estatística de Wald kernelizada

$q_{i} \quad$ Quantil amostral de $\{\mathbf{x}(n)\}_{n=1}^{N}$

$q_{3}-q_{1} \quad$ Distância interquartílica amostral de $\{\mathbf{x}(n)\}_{n=1}^{N}$

$s \quad$ Desvio padrão amostral de $\{\mathbf{x}(n)\}_{n=1}^{N}$

$\mathbf{S}_{\mathbf{X}} \quad$ Matriz de covariância amostral de $\mathbf{X}=\left\{\mathbf{x}_{i}(n)\right\}_{i=1}^{D}{ }_{n=1}^{N}$

$\Phi^{-1}(\cdot) \quad$ Quantil da normal padrão.

$\operatorname{tr}(\cdot) \quad$ Operador traço de matriz

posto $(\cdot) \quad$ Operador posto de matriz

$\operatorname{vec}(\cdot) \quad$ Operador empilhamento das colunas (ou "vetorização") de matriz

$\mathbf{A} \otimes \mathbf{B} \quad$ Produto de Kronecker entre as matrizes $\mathbf{A}$ e $\mathbf{B}$

$\delta_{i j} \quad$ Delta de Kronecker

${ }_{\iota} \pi_{i j}(f) \quad$ Coerência parcial direcionada-informacional ( $i$ PDC)

${ }_{\kappa \eta} \pi_{i j}(f) \quad$ Coerência parcial direcionada-kernelizada-não-linear (knPDC)

${ }_{\iota} \gamma_{i j}(f) \quad$ Função de transferência direcionada-informacional ( $\left.i \mathrm{DTF}\right)$

${ }_{k \eta} \gamma_{i j}(f) \quad$ Função de transferência direcionada-kernelizada-não-linear (knDTF) 
$\mathrm{GCT}_{i j} \quad$ Teste de causalidade de Granger (GCT)

$k_{n} \mathrm{GCT}_{i j} \quad$ Teste de causalidade de Granger-kernelizada-não-linear (knGCT)

$H_{\alpha}(X) \quad$ Entropia de Rényi de $X$

$H(X) \quad$ Entropia de Shannon de $X$

$V_{\alpha}(X) \quad$ Potencial- $\alpha$ de informação de $X$

$H_{n}(\cdot) \quad$ Operador de Volterra de grau $n$

$h_{i}(n) \quad$ Representação de um sistema no domínio do tempo

$h^{(i)}\left(t, \tau_{1}, \cdots, \tau_{i}\right) \quad$ Kernel de Volterra, variante no tempo

$h^{(i)}\left(\tau_{1}, \cdots, \tau_{i}\right) \quad$ Kernel de Volterra, invariante no tempo

$\{w(n)\},\{x(n)\} \quad$ Entrada e saída de um mapa (sistema dinâmico), respectivamente

$g(\cdot) \quad$ Não-linearidade estática

$g_{i}(\cdot) \quad$ Função de base, não-linear

$f(\cdot) \quad$ Função não linear

$p \quad$ Ordem de um modelo

$N_{p, m} \quad$ Número de coeficiente de uma estrutura de Volterra

$k_{i} \quad$ Memória de uma variável, por exemplo, $x_{i}\left(n-k_{i}\right)$

$\tau_{i} \quad$ Memória de um núcleo de Volterra 
SUMÁRIO

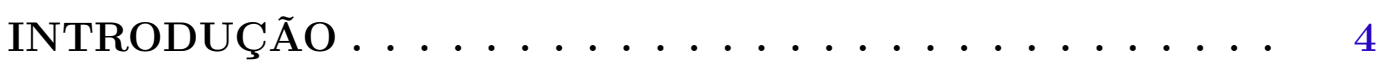

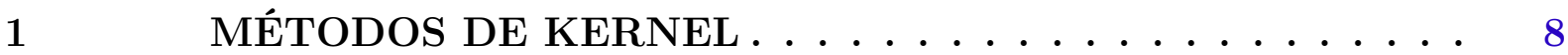

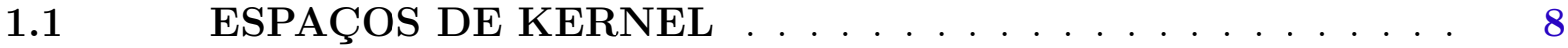

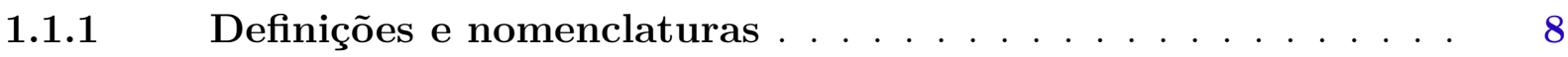

1.1.2 Reproducing kernels . . . . . . . . . . . . . . . 9

1.1.3 Reproducing kernels Hilbert spaces . . . . . . . . . . . . . . 11

1.1.4 O Teorema da Representação de Mercer . . . . . . . . . . . . 11

1.1.5 Propriedade da aproximação universal . . . . . . . . . . . . . 14

1.1.6 Reproducing kernel para funções vetoriais . . . . . . . . . 16

1.1.7 Kernels mais usados . . . . . . . . . . . . . . . . . . 17

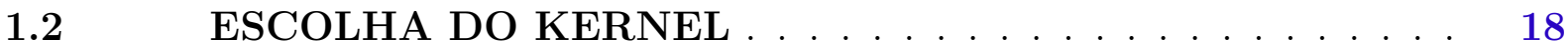

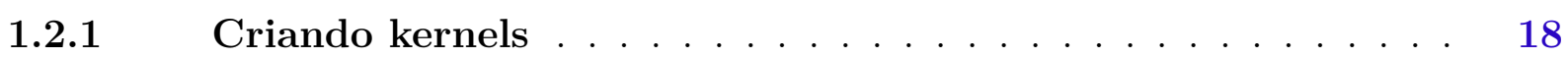

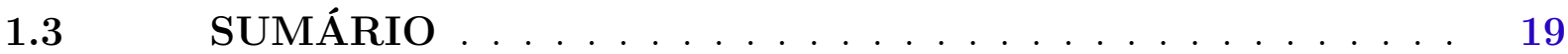

2 MÉTODOS DE KERNEL APLICADOS A MEDIDAS DE

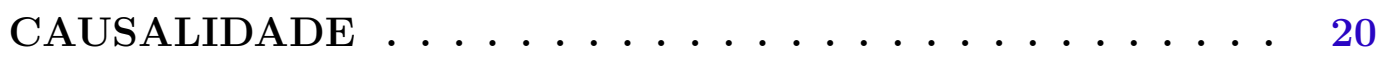

2.1 CAUSALIDADE E SUA INFERÊNICA . . . . . . . . 20

2.2 MODELO AUTORREGRESSIVO VETORIAL KERNELIZADO 21

2.2.1 Estimando o processo autorregressivo kernelizado . . . . . . 22

2.2.2 Propriedades assintóticas dos mínimos quadrados kernelizado 24

2.2.3 Seleção da ordem e adequação do modelo . . . . . . . . . . . 26

2.2.3.1 Seleção da ordem do modelo . . . . . . . . . . . . . 27

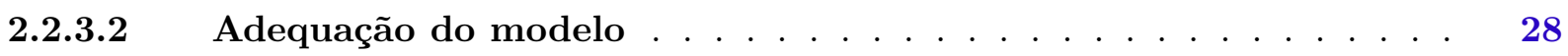

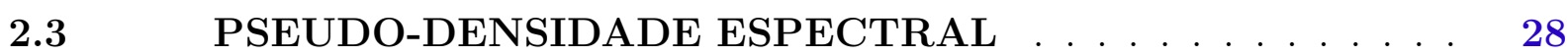

2.3.1 Matriz densidade espectral kernelizada . . . . . . . . . . . 29

2.4 DESCRITORES DE CONECTIVIDADE KERNELIZADOS . 30

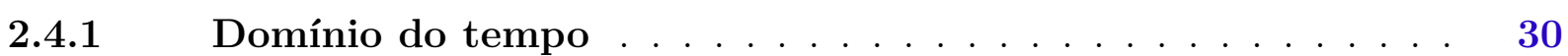

2.4 .2 Domínio da frequência . . . . . . . . . . . . . . . 31

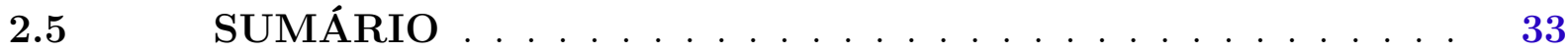

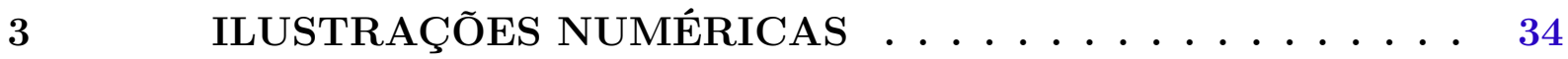

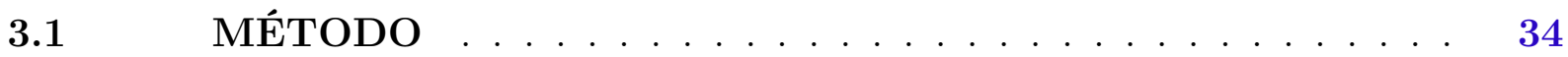

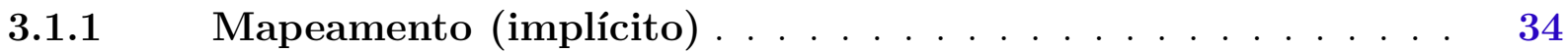

3.1.2 Análise de causalidade . . . . . . . . . . . . . . 35

3.2 CONVENÇÕES PARA APRESENTAR A $(k n)$ DTF $/(k n)$ PDC 35 
3.3 VALIDAÇÃO ESTATÍSTICA DO MÉTODO PROPOSTO . 35

3.3.1 Exemplo 1: Modelo autorregressivo de ordem um (VAR(1)) 3D 37

3.3.2 Exemplo 2: Modelo não-linear de banda limitada 2D $\ldots . .42$

3.3.3 Exemplo 3: Modelo não-linear de banda larga 2D . . . . . . 48

3.3.4 Exemplo 4: Modelo não-linear de banda larga 3D . . . . . . . 55

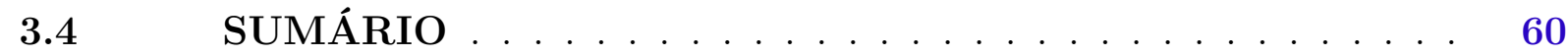

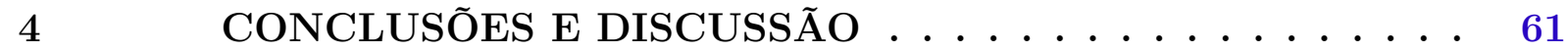

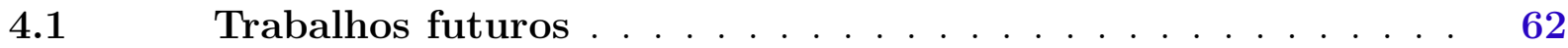

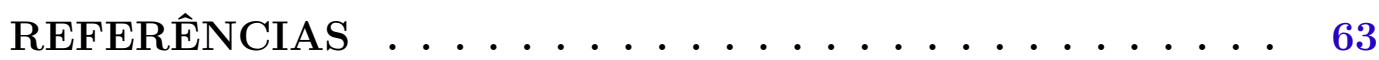

APÊNDICE A - MÍNIMOS QUADRADOS KERNELIZADOS 71

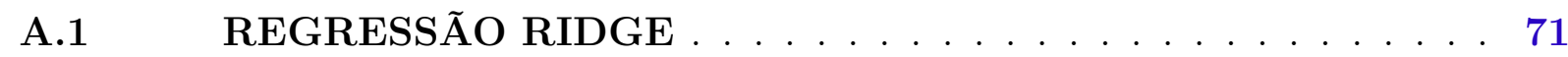

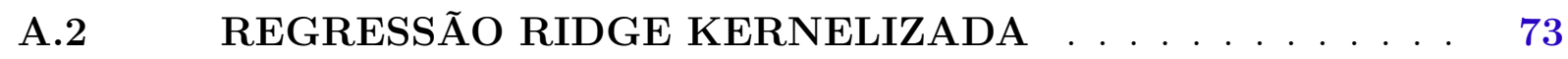

APÊNDICE B - TEORIA DA INFORMAÇÃO . . . . . . 74

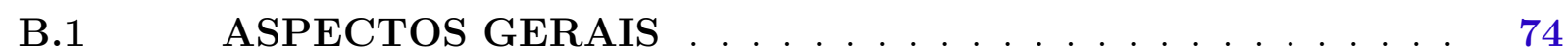

B.2 ESTIMANDO A ENTROPIA . . . . . . . . . . . 75

B.2.1 Propriedades do estimador não-paramétrico de densidade por kernel . . . . . . . . . . . . . . . . . . . . . . . 76

APÊNDICE C - SÉRIES DE VOLTERRA . . . . . 78

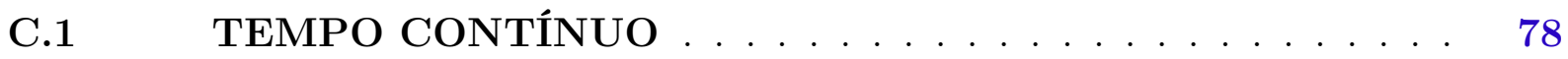

C.2 TEMPO DISCRETO (CASO ESTACIONÁRIO) . . . . . . 79

C.2.1 Sobre a convergência . . . . . . . . . . . . . . . . 80

APÊNDICE D - MODELOS ORIENTADOS À BLOCOS . . 81

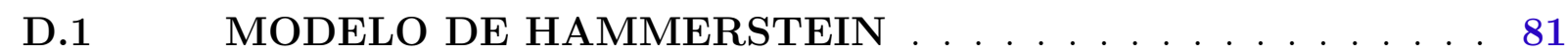

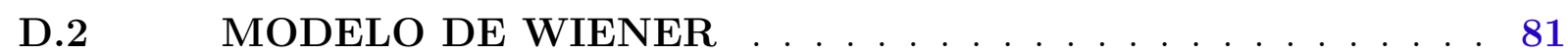

D.3 MODELO DE WIENER-HAMMERSTEIN . . . . . . . . 82

APÊNDICE E - MODELOS NARMAX . . . . . . 83

E.1 MODELOS NÃO-LINEARES DE REGRESSÃO ESTOCÁS-

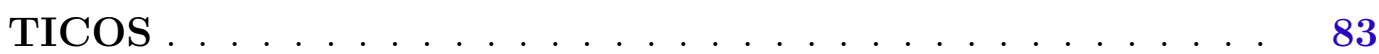

E.1.1 Relação dos modelos NARMAX/NARX com as séries de Vol-

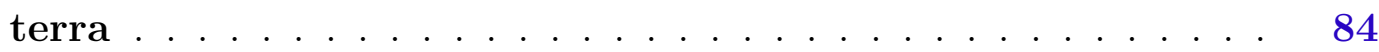

ANEXO A - LISTA DE PUBLICAÇÕES . . . . . . . . 85

A.1 SINAPE $2014 \ldots \ldots \ldots \ldots \ldots \ldots \ldots$ 
A.2 ISI $2015 \ldots \ldots \ldots \ldots \ldots$

A.3 ESTE $2015 \ldots \ldots \ldots \ldots$

A.4 EMBC $2015 \ldots \ldots \ldots \ldots$

A.5 EMBC $2016 \ldots \ldots \ldots \ldots$ 


\section{INTRODUÇÃO}

Séries temporais podem ser vistas como registros de medidas sequenciais no tempo ligadas a quantificadores mensuráveis. Por essa razão, encontram-se no centro de estudos voltados à caracterização da evolução temporal de grandezas em diversas áreas do conhecimento [1-6].

Recentemente, houve um aumento de interesse no estudo que se convencionou chamar de Conectividade Cerebral [7-9]. Segundo extensa revisão contida em [9], há diversas maneiras de se estimar a conectividade entre regiões neurais. Essa pode ser feita usando séries temporais de, por exemplo, eletroencefalograma (Electroencephalogram EEG), ressonância magnética nuclear funcional (Functional Magnetic Resonance Imaging - fMRI), ou, ainda, magnetoencefalograma (Magnetoencephalogram - MEG). Do ponto de vista metodológico, porém, a forma de análise que possui excepcional apelo é a de modelamento por Causalidade de Granger (CG), que é uma propriedade de uma conjunto de dados (com ordem temporal) $x(n)$ possui sobre outra série $y(n)$, quando o passado do primeiro é útil para prever a segunda $[7,10,11]$.

Tradicionalmente muito do esforço para compreender fenômenos físicos/biológicos tem se voltado para o estudo de sua modelagem matemática [12]. Nesse contexto, o que se faz é uma idealização simples dos mapas (sistemas dinâmicos), a fim de descrever algumas dessas características.

Em processamento de sinais neurofisiológicos, recentemente conheceram-se propostas que usam modelos lineares vetoriais autorregressivos (Vector Autoregressive - VAR) de forma intensa. Tal abordagem é interessante, pois permite representar a CG no domínio da frequência. Exemplo disso se dá por meio da Função de Transferência Direcionada [8] (Directed Transfer Function - DTF) e da Coerência Parcial Direcionada [7] (Partial Directed Coherence - PDC).

No estudo realizado em [13], o presente autor mostrou ser possível capturar a presença de interações não-lineares, por meio de uma abordagem híbrida, dita Mapeamento por Entropia Deslizante, em que a idéia principal é calcular uma medida de complexidade para uma série temporal, sobre uma janela de longa duração adequada. Isso produz outra sequência, aliada, que retrata como sua entropia evolui com o tempo. O próximo passo consiste na comparação dos resultados desses mapeamentos, utilizando métodos lineares multivariados $[14,15]$.

A dificuldade central do método citado no parágrafo anterior está no passo de mapeamento das séries originais, ao produzir suas correspondentes séries transformadas (conforme é descrito em [13-15]), pois não há garantia formal de que se preservem as 
relações pré-existentes. Dessa forma, buscam-se alternativas eventualmente mais simples para se verificar como ocorre a transferência de informação entre séries [16-21].

A maioria das medidas de conectividade práticas são lineares por natureza a DTF e PDC estão entre elas e provaram ser robustas [22,23], devido às conhecidas propriedades assintóticas dos modelos VAR, incluindo rápida convergência. Essas técnicas têm sido utilizadas com algum sucesso no contexto de modelos não-lineares [24].

Por outro lado, é bem conhecido que os métodos lineares falham quando o acoplamento é de natureza polinomial com potência par. Assim, para lidar com essa deficiência, algumas propostas foram oferecidas [25-31]. Elas compartilham problemas de convergência, necessitando um número grande de amostras, o ajuste de um excessivo número de parâmetros ou, ainda, a necessidade de otimizar funcionais não convexos. Outro problema é a falta de estatísticas assintóticas rigorosas que permitam definir um critério robusto de conectividade, quando comparadas ao caso linear [10,22,23].

Como se sabe, representar um mapa (sistema dinâmico) não-linear de maneira satisfatória, não é uma tarefa simples. Para tanto, foram desenvolvidas várias idéias. Algumas são bastante gerais e outras mais restritas (quanto às aplicações), sendo válidas somente em casos mais específicos.

A resolução de um problema de Identificação de Sistemas requer que se opte por uma dentre várias representações (ou classes de modelos). Para que a decisão seja adequada, é preciso conhecer as propriedades de cada classe, comparativamente às demais. Assim, um estudo de revisão dessas técnicas foi realizado, com o objetivo de expor as características principais dessas técnicas e por questão de completude do texto, estão contidas nos Apêndices B, C, D e E. Recomenda-se que um estudo comparativo deva ser feito, entre as novas técnicas apresentadas nessa tese e aquelas que estão nos apêndices.

Assim, a proposta central dessa investigação é usar uma forma mais geral de modelamento de mapas (sistemas dinâmicos) não-lineares, garantindo que as séries resultantes possam ser relacionadas de maneira linear.

Entre os mapeamentos, o de maior interesse está o que atende pelo nome de Métodos de Kernel, sugerido por Vapnik [32], ao tratar da teoria de classificadores. Métodos de Kernel consistem em técnicas que exibem menor complexidade e fornecem uma abordagem simples para transformar um problema não-linear em problemas de otimização convexa [33].

A escolha dos métodos de kernel reside no fato, como citado acima, de reduzir um problema não-convexo, em outro convexo. Por isso têm sido usados para modificar (que, no caso, a esse processo dá-se o sugestivo nome de kernelizar) conhecidas técnicas lineares, em suas análogas, porém não-lineares, como, por exemplo, as de separação de sinais ou seja, Análise de Componente Principal [34] e Análise de Componente Independente [35], 
além de predição autorregressiva univariada, como em [36,37].

Na Figura 1 observa-se idéia básica dos métodos de kernel, ou seja, o mapeamento (transformação) dos dados, que estão em um domínio, dito espaço de entrada, em outro de dimensão maior, o espaço de características, tende a descomplicar as relações não-lineares, existentes em sua estrutura, linearizando-as.

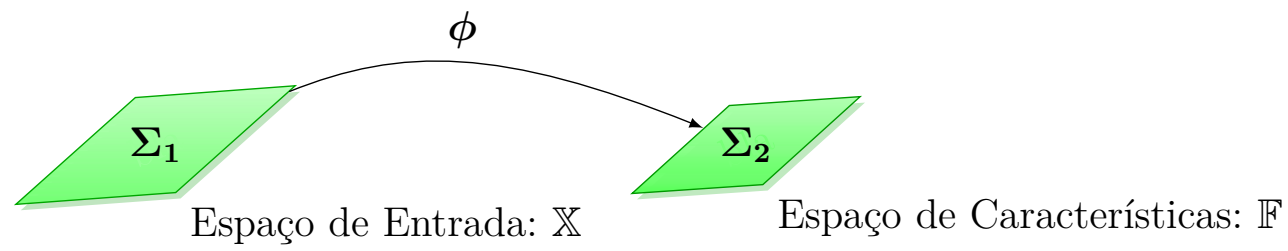

Figura 1 - Mapeamento de características: a idéia básica dos métodos de kernel. O mapeamento $\phi$ transforma o domínio (chamado de espaço de entrada) em outro de maior dimensão, ou seja, no espaço de funções de características. Observa-se que esse mapeamento é capaz de linearizar os dados. $\boldsymbol{\Sigma}_{\mathbf{1}}$ e $\boldsymbol{\Sigma}_{\mathbf{2}}$ representam as estruturas de covariancias dos dados nesses dois espaços.

Portanto, do apresentado, o objetivo desse texto é estudar e desenvolver novos ferramentais estatísticos, apresentando estimadores e seus resultados assintóticos, para que seja possível inferir e classificar sistemas biológicos, que têm, por natureza, comportamento não-linear em suas séries temporais.

A metodologia do trabalho se inicia com estudo dos métodos de kernels, no Capítulo 1, para que seja possível transformar as conhecidas técnicas lineares, de causalidade de Granger, em não-lineares, além um estudo teórico, no Capítulo 2, das estatísticas assintóticas dos novos estimadores. Segue com a validação de tal proposta, no Capítulo 3, simulando-se mapas toys simbólicos de cenários de biológicos, inferindo seu comportamento por meio dos métodos introduzidos, que estendem a representação da causalidade de Granger.

A abordagem dessa tese de doutorado é fundamentalmente numérica e experimental. As conclusões baseiam-se em simulações computacionais, com o auxílio do ambiente MATLAB.

Por uma questão de coesão e coerência, o texto divide-se nos seguintes capítulos,

$\checkmark$ Capítulo 1 Métodos de Kernel — que provê a teoria necessária para o desenvolvimento dos métodos e das técnicas propostas nesta tese;

$\checkmark$ Capítulo 2 Métodos de Kernel Aplicados à Medidas de Causalidade que envolve a generalização/estensão da causalidade de Granger para o caso nãolinear, tanto no domínio do tempo, quanto no da frequência. Para isso, consideram- 
se os métodos de kernel, pois a relação destes últimos com os métodos lineares acima é tácita, em vista do kernel trick, que é a ferramenta matemática, descrita no Capítulo 1, responsável pela kernelização de métodos lineares;

$\checkmark$ Capítulo 3 Ilustrações Numéricas - em que são realizados experimentos numéricos com quatro mapas "toys", para se testar a robustez estatística daquilo que foi proposto no Capítulo 1;

$\checkmark$ Capítulo 4 Conclusões e Discussão — no último capítulo, dá-se um panorama geral da tese, uma breve discussão e trabalhos futuros.

Seguem-se, então, Apêndices de referência. Adicionalmente, no Anexo, encontrase uma lista das publicações resultantes do trabalho de pesquisa. Um desses artigos, foi creditado com o prêmio de "Geographic Finalist: Latin America" na competição de artigos estudantis da " 37 th Annual International Conference of the IEEE Engineering in Medicine and Biology Society".

\section{CONTRIBUIÇÕES}

As principais contribuições foram a estensão, para o caso não-linear, de diversos métodos lineares, a saber,

1. modelos $\operatorname{VAR}(p)$, juntamente com o estimador de mínimos quadrados de seus parâmetros e suas propriedades assintóticas;

2. critérios para escolha de ordem de modelo e qualidade de ajuste;

3. fatoração da matriz densidade espectral;

4. testes para a causalidade de Granger no domínio do tempo e da frequência (DTF e PDC).

Ao novo modelo, dá-se o nome de Autorregressivo Vetorial kernelizado $(k \operatorname{VAR}(p))$ e às novas quantias de medida de fluxo de informação, Teste de Causalidade de Grangerkernelizada-não-linear (knGCT), Função de Transferência Direcionada-kernelizada-nãolinear (knDTF) e Coerência Parcial Direcionada-kernelizada-não-linear (knPDC). 


\section{MÉTODOS DE KERNEL}

Este Capítulo oferece uma visão do quadro teórico usado para kernelizar conhecidas técnicas de causalidade de Granger, tanto no Domínio do Tempo, quanto no da Frequência, permitindo estendê-las ao caso não-linear.

As Seções seguintes apresentam os conceitos fundamentais mais relevantes junto com sua ilustração por meio de exemplos para um melhor entendimento da teoria.

\subsection{ESPAÇOS DE KERNEL}

\subsubsection{Definições e nomenclaturas}

Aqui, introduzem-se algumas definições básicas e nomenclaturas ${ }^{1}[33,38]$. Portanto, tem-se a seguinte

Definição 1. (Kernel Positivo Semi-Definido.) Seja $X$ um subconjunto do $\mathbb{R}^{N}$. Uma função contínua e simétrica $\kappa: \mathbb{X} \times \mathbb{X} \rightarrow \mathbb{R}$ que opera sobre dados, nesse prórprio espaço $\mathbb{X}$ (denominado de espaço de entrada) é chamada de positiva semi-definida (ou, ainda, kernel de Mercer [39]), se para qualquer desses dados $\{\mathbf{x}(n)\}_{n=1}^{N}$ for satisfeita a relação

$$
\sum_{i=1}^{N} \sum_{j=1}^{N} \alpha_{i} \alpha_{j} \kappa(\mathbf{x}(i), \mathbf{x}(j)) \geq 0 \text {, para quaisquer } \alpha_{n} \text { em } \mathbb{R}
$$

e

Definição 2. (Matriz de Kernel.) Para um conjunto de dados de entrada $\{\mathbf{x}(n)\}_{n=1}^{N} a$ matriz de kernel é dada por,

$$
\mathbf{K}=\left[K_{i j}\right]=[\kappa(\mathbf{x}(i), \mathbf{x}(j))]
$$

para $i, j=1, \cdots, N$.

Agora, em vista das Definições 1 e 2, tem-se a

Definição 3. (Matriz de Kernel Positiva Semi-Definida.) Uma forma quadrática para K, dada por

$$
\sum_{i=1}^{N} \sum_{j=1}^{N} \alpha_{i} \alpha_{j} K_{i j} \geq 0, \text { para quaisquer } \alpha_{n} \text { em } \mathbb{R},
$$

é chamada de matriz de kernel positiva semi-definida.

$\overline{1}$ No presente texto usam-se alguns termos em inglês, pois, na literatura, não há um consenso sobre sua tradução. 


\subsubsection{Reproducing kernels}

Primeiramente, note que aqui é necessário começar com a definção de espaço de características. Denotado por $\mathbb{F}$, é o contradomínio dos mapeamentos usados neste texto, reresenta um espaço vetorial dotado de produto interno e completo. Portanto, trata-se de um espaço de Hilbert e sua dimensão será dada mais adiante.

Pode-se mostrar [33] que um espaço de características $\mathbb{F}$ está associado a um kernel positivo semi-definido, de tal forma que este é um produto interno em tal espaço. A fim de se construir o espaço de características, primeiramente seja o mapeamento de características, de $\mathbb{X}\left(\right.$ no $\mathbb{R}^{N}$ ) sobre um espaço de funções $\mathbb{F}$ (no $\mathbb{R}^{M}$, com $M \gg N$ ), para um dado kernel positivo semi-definido $\kappa(\cdot, \cdot)$, ou seja,

$$
\begin{aligned}
\phi \quad: \quad \mathbb{X} & \longrightarrow \mathbb{F} \\
\mathbf{x} & \longmapsto \kappa(\mathbf{x}, \cdot)
\end{aligned}
$$

Portanto, a função $\phi(\mathbf{x})(\cdot)$ atribui o valor $\kappa(\mathbf{x}, \mathbf{y})$, para um dado de entrada $\mathbf{y}$ e, assim, pode-se interpretar o kernel como uma função de similaridade, já que o mapeamento (1.4) representa cada ponto de entrada $\mathbf{x}$ por sua similaridade $\boldsymbol{\phi}(\mathbf{x})(\cdot)=\kappa(\mathbf{x}, \cdot)$, para todos os outros pontos do domínio $\mathbb{X}$.

Logo, para se construir um espaço de características associado a $\phi$, a imagem de $\phi$ deve se tornar um espaço vetorial dotado de produto interno [33]. Para tornar isso factível, sejam, então, duas funções $f(\cdot)$ e $g(\cdot)$ que obedeçam às leis de formação,

$$
f(\cdot)=\sum_{i=1}^{l} \alpha_{i} \kappa(\mathbf{x}(i), \cdot) \text {, para quaisquer } \alpha_{n} \text { em } \mathbb{R}
$$

$\mathrm{e}$

$$
g(\cdot)=\sum_{j=1}^{m} \beta_{j} \kappa(\mathbf{y}(j), \cdot), \text { para quaisquer } \beta_{n} \text { em } \mathbb{R},
$$

em que ambos $\{\mathbf{x}(i)\}_{i=1}^{l}$ e $\{\mathbf{y}(j)\}_{j=1}^{m}$ estão no domínio $\mathbb{X}$. Daí, defina o produto interno $\langle\cdot, \cdot\rangle$, como

$$
\langle f(\cdot), g(\cdot)\rangle=\sum_{i=1}^{l} \sum_{j=1}^{m} \alpha_{i} \beta_{j} \kappa(\mathbf{x}(i), \mathbf{y}(j)),
$$

que é uma forma quadrática (bilinear), satisfazendo as propriedades usuais do produto interno, ou seja,

1. Simetria: $\langle f, g\rangle=\langle g, f\rangle$;

2. Linearidade: $\langle(a f+b g), h\rangle=a\langle f, h\rangle+b\langle g, h\rangle$, para algum $a$ e $b$ em $\mathbb{R}$.

3. Norma quadrática: $\|f\|_{2}^{2}:=\langle f, f\rangle \geq 0 \Longleftrightarrow f \geq 0$. 
4. Propriedade reprodutiva (Reproducing property): $\langle f(\cdot), \kappa(\mathbf{y}, \cdot)\rangle=f(\mathbf{y})$. Observe que para se mostrar esse fato, basta fazer $g(\cdot)=\kappa(\mathbf{y}, \cdot)$ e substituir em (1.5), obtendo-se

$$
\begin{aligned}
\langle f(\cdot), g(\cdot)\rangle=\langle f(\cdot), \kappa(\mathbf{y}, \cdot)\rangle & =\sum_{i=1}^{l} \alpha_{i} \kappa(\mathbf{x}(i), \mathbf{y}) \\
& =f(\mathbf{y})
\end{aligned}
$$

Note que, pelos Itens 1,2 e 3 , agora tem-se estabelecido que $\langle\cdot, \cdot\rangle$ de fato é um produto interno.

Da propriedade do Item 4 , vê-se que $\kappa(\cdot, \cdot)$ é capaz de representar a função $f(\cdot)$ no espaço de características $\mathbb{F}$. Seguindo (1.6), é fácil ver que,

$$
\langle\kappa(\mathbf{x}, \cdot), \kappa(\mathbf{y}, \cdot)\rangle=\kappa(\mathbf{x}, \mathbf{y})
$$

o qual, segundo a Definição 1 é positivo semi-definido e, portanto, pode ter um espaço vetorial de características com produto interno associado, permitindo que $\kappa(\mathbf{x}, \mathbf{y})$ possa ser reescrito como,

$$
\kappa(\mathbf{x}, \mathbf{y})=\langle\phi(\mathbf{x}), \phi(\mathbf{y})\rangle .
$$

Um exemplo para o melhor entendimento da argumentação anterior é exposto a seguir, na Seção 1.1.4.

Na literatura, o procedimento de escrever o kernel positivo semi-definido como um produto interno, é conhecido como kernel trick $[16,33,38]^{2}$.

O kernel trick diz que a partir de uma técnica, baseada em produto interno, é possível construir outra (paralela e da mesma classe) apenas substituindo o produto interno por um kernel adequado.

Considerando a observação do parágrafo acima, a idéia principal dos procedimentos propostos nessa tese baseia-se no que é chamado de "kernelização". Como as técnicas de causalidade de Granger são baseadas em produto interno linear [10, 22, 23], pode-se transformá-las em técnicas não-lineares, pela substituição do produto interno por um kernel não-linear.

Entretanto, a interpretação do método não é tão simples, pois não há um mapeamento de forma direta do dados. Com o kernel trick, essa operação é feita de forma indireta e implicita. Fazer isso de forma direta é uma tarefa muito difícil e, às vezes, impossível, devido à alta dimensionalidade do espaço de características.

2 É possível provar que um kernel pode ser obtido de (1.8) para todo mapeamento (linear ou não), de um espaço de entrada para outro de características (para a prova, o leitor deve referir-se a [33], p. $37-40)$ 
Thomas M. Cover [40] provou que a operação de mapeamento de um espaço para outro de maior dimensão pode ser eficiente, em problemas de classificação. Em seu Teorema, Cover diz que ao aumentar a dimensão do espaço é mais provável que haja separações lineares e o número de possíveis separações é dado por $C(N, k)=2 \sum_{k=0}^{n-1}\left(\begin{array}{c}N-1 \\ k\end{array}\right)$, em que $N$ é o número de amostras do conjunto em um espaço $\mathbb{R}^{n}$. Porém, se $n>N$ então todas as $2^{N}$ separações são possíveis.

\subsubsection{Reproducing kernel Hilbert spaces}

Considere agora, espaços de Hilbert munidos de produto interno. Formalmente [2,38] um espaço de Hilbert $\mathbb{H}$ com produto interno é completo se toda sequência de vetores de Cauchy convergir para um limite em $\mathbb{H}$. Portanto, usando os conceitos apresentados na Seção 1.1.2, tem-se a

Definição 4. (Reproducing kernel Hilbert spaces) Seja $\mathbb{X}$, um espaço de entrada e o espaço de características $\mathbb{F}$ de funções $(f: \mathbb{X} \rightarrow \mathbb{R})$ de Hilbert $\mathbb{H}$. Então $\mathbb{H}$ é chamado de "Reproducing kernel Hilbert space" (RKHS), com produto interno $\langle\cdot, \cdot\rangle$ e norma $\|f(\cdot)\|_{2}^{2}=$ $\langle f(\cdot), f(\cdot)\rangle$, se existir uma função $\kappa: \mathbb{X} \times \mathbb{X} \rightarrow \mathbb{R}$ tal que,

1. $\kappa(\cdot, \cdot)$ possui a propriedade reprodutiva, i.e.,

$$
\langle f(\cdot), \kappa(\mathbf{x}, \cdot)\rangle=f(\mathbf{x}) \text {, para alguma } f \text { em } \mathbb{H} \text { e quaisquer } \mathbf{x} \text { em } \mathbb{X},
$$

$e\langle\kappa(\mathbf{x}, \cdot), \kappa(\mathbf{y}, \cdot)\rangle=\kappa(\mathbf{x}, \mathbf{y})$

2. $\kappa(\cdot, \cdot)$ gera $\mathbb{H}$, i.e.,

$$
f(\cdot)=\sum_{i=1}^{l} \alpha_{i} \kappa(\mathbf{x}(i), \cdot) \text {, para alguma } f \text { em } \mathbb{H} \text { e quaisquer } \alpha_{n} \text { em } \mathbb{R} .
$$

Na Seção seguinte, será mostrado que de um kernel obtém-se um único RKHS, mas o contrário não é verdadeiro.

\subsubsection{O Teorema da Representação de Mercer}

Aqui, expõe-se a versão do Teorema de Mercer de [41], que também pode ser encontrado em $[16,33,38,39]$ e diz que qualquer kernel em um RKHS possui uma decomposição espectral.

Note a necessidade de se assumir que $(\mathbb{X}, \mu)$ seja um espaço de medida finita ${ }^{3}$. O termo para quase todo, usado abaixo, significa exceto para conjuntos de medida zero

3 Um espaço de medida finita é um conjunto $\mathbb{X}$ com uma $\sigma$-álgebra definida neste e uma medida definida no último, satisfazendo $\mu(\mathbb{X})>0$ (tal que, através de um fator de escala, $\mu$ é uma medida de probablidade.) 
(para maiores detalhes, refira-se ao Apêndice B, seção B.3 de [33]). Utilizando a medida de Lesbegue-Borel usual, pode-se simplificar a notação adiante e escrever $\mathrm{d} \mu(\mathbf{x})$ como $\mathrm{d} \mathbf{x}$ e, também, dizer que $\mathbb{X}$ é um subconjunto compacto de $\mathbb{R}^{N}$. Para maiores detalhes, envolvendo explicações sobre os termos empregados no Teorema 1, o leitor deve referir-se ao Apêndice B, seção B.3 de [33].

Teorema 1. (Teorema de Mercer) Sejam $\mathbb{X}$ um subconjunto compacto de $\mathbb{R}$, o espaço $L^{2}(\mathbb{X})$, o conjunto das funções quadrado integráveis, i.e., $\int_{\mathbb{X}} f^{2}(\mathbf{x}) \mathrm{d} \mathbf{x}<\infty$, para toda $f(\cdot)$ em $L^{2}(\mathbb{X})$ e o espaço $L^{\infty}(\mathbb{X})$, o conjunto das funções absolutamente integráveis, ou seja, $\int_{\mathbb{X}}|f(\mathbf{x})| \mathrm{d} \mathbf{x}<\infty$, para toda $f(\cdot)$ em $L^{\infty}(\mathbb{X})$.

Agora, suponha que $\kappa(\cdot, \cdot)$, pertencente a $L^{\infty}(\mathbb{X} \times \mathbb{X})$, seja uma função simétrica a valores reais, tal que o operador integral

$$
\begin{aligned}
T_{\kappa}: L^{2}(\mathbb{X}) & \longrightarrow L^{2}(\mathbb{X}) \\
\left(T_{\kappa} f\right)(\mathbf{x}) & \longmapsto \int_{\mathbb{X}} \kappa(\mathbf{x}, \mathbf{y}) f(\mathbf{y}) \mathrm{d} \mu(\mathbf{y})
\end{aligned}
$$

é positivo semi-definido, ou seja, para toda $f(\cdot)$ em $L^{2}(\mathbb{X})$, tem-se que,

$$
\int_{\mathbb{X} \times \mathbb{X}} \kappa(\mathbf{x}, \mathbf{y}) f(\mathbf{x}) f(\mathbf{y}) \mathrm{d} \mu(\mathbf{x}) \mathrm{d} \mu(\mathbf{y}) \geq 0 .
$$

Sejam, também, as autofunções ortonormais $\varphi_{i}(\cdot)$ em $L^{2}(\mathbb{X})$ de $T_{\kappa}$, associadas aos autovalores $\lambda_{i}>0$, classificados em ordem decressente.

Então,

1. $\left(\lambda_{i}\right)_{i}$ pertence $a \ell_{1}$;

2. a decomposição espectral

$$
\kappa(\mathbf{x}, \mathbf{y})=\sum_{i=1}^{N_{\mathbb{H}}} \lambda_{i} \varphi_{i}(\mathbf{x}) \varphi_{i}(\mathbf{y})
$$

é válida para quase todo $(\mathbf{x}, \mathbf{y})$. Aqui, $N_{\mathbb{H}}$ pode pertencer a $\mathbb{N}$ ou tender a $\infty$. No último caso, a série (1.13) converge absolutamente e uniformemente para quase todo $(\mathbf{x}, \mathbf{y})$.

A prova do Teorema 1 pode ser encontrada em [42].

Logo, da afirmação 2 (do Teorema 1 ), segue que $\kappa(\mathbf{x}, \mathbf{y})$ corresponde a um produto interno em $\ell_{2}^{N_{\mathbb{H}}}$, pois $\kappa(\mathbf{x}, \mathbf{y})=\langle\phi(\mathbf{x}), \phi(\mathbf{y})\rangle$, em que

$$
\begin{aligned}
\phi: \mathbb{X} & \longrightarrow \ell_{2}^{N_{\mathbb{H}}} \\
\mathbf{x} & \longmapsto\left\{\sqrt{\lambda_{i}} \varphi_{i}(\mathbf{x})\right\}_{i=1}^{N_{\mathbb{H}}}
\end{aligned}
$$

e, por essa exposição, é claro que a dimensionalidade de $\mathbb{F}$ fica determinada pelo número de autovalores. 
Para ajudar no entendimento do Teorema 1, um exemplo simples se faz útil: considere um kernel polinomial, de grau dois, e.g.,

$$
\kappa(\mathbf{x}, \mathbf{y})=(c+\langle\mathbf{x}, \mathbf{y}\rangle)^{2},
$$

em que $\mathbf{x}=\left[x_{1}, x_{2}\right]^{\top}, \mathbf{y}=\left[y_{1}, y_{2}\right]^{\top}$ e $c$ está em $\mathbb{R}$. Fazendo a expansão do kernel,

$$
\begin{aligned}
\kappa(\mathbf{x}, \mathbf{y}) & =c^{2}+\left(x_{1} y_{1}\right)^{2}+\left(x_{2} y_{2}\right)^{2}+2 c\left(x_{1} y_{1}+x_{2} y_{2}\right)+2 x_{1} x_{2} y_{1} y_{2}, \\
& =c c+\sqrt{2 c} x_{1} \sqrt{2 c} y_{1}+\sqrt{2 c} x_{2} \sqrt{2 c} y_{2}+x_{1}^{2} y_{1}^{2}+x_{2}^{2} y_{2}^{2}+\sqrt{2} x_{1} x_{2} \sqrt{2} y_{1} y_{2} \\
& =\langle\boldsymbol{\phi}(\mathbf{x}), \boldsymbol{\phi}(\mathbf{y})\rangle=\boldsymbol{\phi}(\mathbf{x})^{\top} \boldsymbol{\phi}(\mathbf{y}),
\end{aligned}
$$

$\operatorname{com} c>0$,

$$
\phi(\mathbf{x})=\left[c, \sqrt{2 c} x_{1}, \sqrt{2 c} x_{2}, x_{1}^{2}, x_{2}^{2}, \sqrt{2} x_{1} x_{2}\right]^{\top}
$$

e

$$
\phi(\mathbf{y})=\left[c, \sqrt{2 c} y_{1}, \sqrt{2 c} y_{2}, y_{1}^{2}, y_{2}^{2}, \sqrt{2} y_{1} y_{2}\right]^{\top}
$$

Esse exemplo deixa claro que existe um RKHS $\mathbb{H}$ associado com o kernel de Mercer $\kappa(\mathbf{x}, \mathbf{y})=(c+\langle\mathbf{x}, \mathbf{y}\rangle)^{2}$ e isso é válido para qualquer kernel de Mercer. Para visualizar isso, considere um kernel positivo semi-definido e um espaço de Hilbert que contenha funções da forma

$$
f(\mathbf{x})=\sum_{i=1}^{\infty} \alpha_{i} \sum_{j=1}^{N_{\mathbb{H}}} \lambda_{j} \varphi_{j}(\mathbf{x}) \varphi_{j}(\mathbf{x}(i))
$$

Então, por linearidade,

$$
\langle f(\cdot), \kappa(\cdot, \mathbf{y})\rangle=\sum_{i=1}^{\infty} \alpha_{i} \sum_{j=1}^{N_{\mathbb{H}}} \sum_{k=1}^{N_{\mathbb{H}}} \lambda_{j} \varphi_{j}(\mathbf{x}(i))\left\langle\varphi_{j}, \varphi_{k}\right\rangle \varphi_{j}(\mathbf{y})
$$

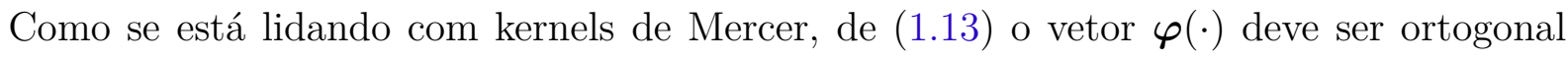
com respeito ao produto interno $\langle\cdot, \cdot\rangle$, em $L^{2}(\mathbb{X})$ e, portanto, uma escolha canônica seria $\left\langle\varphi_{j}, \varphi_{k}\right\rangle=\frac{\delta_{i j}}{\lambda_{j}}$ (com $\delta_{i j}$ o símbolo para o delta de Kronecker). Assim, substituindo na equação acima,

$$
\begin{aligned}
\langle f(\cdot), \kappa(\cdot, \mathbf{y})\rangle & =\sum_{i=1}^{\infty} \alpha_{i} \sum_{j=1}^{N_{\mathbb{H}}} \varphi_{j}(\mathbf{x}(i)) \varphi_{j}(\mathbf{y}), \\
& =\sum_{i=1}^{\infty} \kappa(\cdot, \mathbf{y}) \\
& =f(\mathbf{y}),
\end{aligned}
$$

que é a propriedade reprodutiva.

Na próxima seção será mostrada uma importante propriedade que lida com o truncamento das séries vistas acima. 


\subsubsection{Propriedade da aproximação universal}

Para falar sobre a propriedade da aproximação universal considere um problema de projetar um espaço de entrada [38]. Logo, sejam os dados desse espaço $\mathbf{x}=\left[x_{1}, x_{2}\right]^{\top}$ e a função que se deseja aproximar $f(\mathbf{x})=a_{1} x_{1}+a_{2} x_{2}+a_{3} x_{1}^{2}+a_{4} x_{2}^{2}$, com $\left\{a_{1}, a_{2}, a_{3}, a_{4}\right\}$ constantes reais. Nota-se que o uso de um sistema linear, para aproximar $f(\cdot)$ por uma combinação linear de $u_{1}$ e $u_{2}$, não é capaz de reproduzir as estruturas dos dados do modelo, como foram postas. Para contornar esse problema, considere o uso de um RKHS, mais especificamente um espaço de características de Mercer, através do kernel (1.15). Com ele, é possível linearizar o modelo, utilizando o mapa não-linear,

$$
\begin{aligned}
\phi: \mathbb{X} \subset \mathbb{R}^{2} & \longrightarrow \mathbb{F} \subset \mathbb{R}^{6} \\
{\left[x_{1}, x_{2}\right]^{\top} } & \longmapsto\left[\phi_{1}, \phi_{2}, \phi_{3}, \phi_{4}, \phi_{5}, \phi_{6}\right]^{\top}=\left[c, \sqrt{2 c} x_{1}, \sqrt{2 c} x_{2}, x_{1}^{2}, x_{2}^{2}, \sqrt{2} x_{1} x_{2}\right]^{\top}
\end{aligned}
$$

e, portanto, $f(\mathbf{x})$ fica reescrita como

$$
f(\phi(\mathbf{x}))=\left[0, \frac{a_{1}}{\sqrt{2 c}}, \frac{a_{2}}{\sqrt{2 c}}, a_{3}, a_{4}, 0\right]^{\top} \phi(\mathbf{x}) .
$$

Através desse exemplo fica claro que o espaço de características $\mathbb{F}$ contém redundância e pode, facilmente, ficar infactível de ser realizável computacionalmente, já que o $\mathbb{F}$ possui dimensão dada por [43],

$$
\operatorname{dim} \mathbb{F}=\left(\begin{array}{c}
N+d-1 \\
d
\end{array}\right),
$$

em que $N=\operatorname{dim} \mathbb{X}$ e $d$ é a ordem do kernel monomial (se o kernel for polinomial, deve-se modificar $d$ para $d+1$, devido ao termo independente).

Assim, no exemplo acima, as primeira e última componentes do vetor $\phi(\mathbf{x})$ fazem com que este seja linearmente dependente (l.d.) e, portanto, pode ter sua dimensionalidade reduzida. Logo, alguns problemas intrínsecos aos métodos de kernels surgem desse argumento, pois é possível optar por colocar quantas dimensões de características quanto se queira, a fim de se tentar refletir a estrutura dos dados do modelo, mas esse pensamento é enganoso, pois pode levar ao perigo do sobremodelamento [38], ou seja, o modelo de $f(\mathbf{x})$ no espaço de características possui dimensões redundantes, que podem ser suprimidas, através de algum critério.

Assim, surge a idéia de seleção de dimensões de características, para que a dimensionalidade seja reduzida. Para tanto, utiliza-se a técnica de regularização, detalhada adiante.

Pode-se provar [44] que existem diversos tipos de kernels, podendo aproximar arbitrariamente bem qualquer função contínua. Isso é conhecido como a propriedade da 
aproximação universal. Formalmente, para todo mapeamento contínuo $f: \mathbb{X} \rightarrow \mathbb{R}$ e qualquer $\varepsilon>0$, para um conjunto de dados $\{\mathbf{x}(i)\}_{i=1}^{l}$ em $\mathbb{X}$, existem: (a) números reais $\left\{\alpha_{i}\right\}_{i=1}^{l}$ e (b) um kernel $\kappa(\cdot, \cdot)$, tais que,

$$
\left\|f(\cdot)-\sum_{i=1}^{l} \alpha_{i} \kappa(\mathbf{x}(i), \cdot)\right\|_{2} \leq \varepsilon
$$

Agora, se for definido um vetor $\boldsymbol{\psi}$, no espaço de características $\mathbb{F}$, como $\boldsymbol{\psi}=$ $\sum_{i=1}^{l} \alpha_{i} \phi(\mathbf{x}(i))$, então pelo kernel trick $(1.8)$ e (1.22), vale a aproximação universal em $\mathbb{F}$ para o modelo de $f(\cdot)$, ou seja,

$$
\begin{aligned}
\left\|f(\cdot)-\boldsymbol{\psi}^{\top} \boldsymbol{\phi}(\cdot)\right\|_{2} & =\left\|f(\cdot)-\left[\sum_{i=1}^{l} \alpha_{i} \boldsymbol{\phi}(\mathbf{x}(i))\right]^{\top} \boldsymbol{\phi}(\cdot)\right\|_{2} \\
& =\left\|f(\cdot)-\sum_{i=1}^{l} \alpha_{i}\left[\boldsymbol{\phi}(\mathbf{x}(i))^{\top} \boldsymbol{\phi}(\cdot)\right]\right\|_{2} \\
& =\left\|f(\cdot)-\sum_{i=1}^{l} \alpha_{i} \kappa(\mathbf{x}(i), \cdot)\right\|_{2} \leq \varepsilon .
\end{aligned}
$$

Com a finalidade de se determinar o conjunto de pontos $\{f(\mathbf{x})\}$, para a aproximação universal em (1.22) ser factível, deve-se fazer uso do Teorema da Representação de Mercer $[45]^{4}$. Como o objetivo do problema é minimizar uma função quadrática regularizada, então,

$$
\min _{f(\mathbf{x}) \in \mathbb{H}}\{J(f(\mathbf{x}))\}=\min _{f(\mathbf{x}) \in \mathbb{H}}\left\{\sum_{i=1}^{N}\left(y_{i}-f\left(\mathbf{x}_{i}\right)\right)^{2}+\lambda\left\|f\left(\mathbf{x}_{i}\right)\right\|_{2}^{2}\right\} .
$$

Antes de continuar, cabem algumas observações.

Note que a escolha do erro quadrático regularizado em (1.24) foi arbitrário, sendo possível adotar outra forma de funcional. Porém, é comum o uso de uma função custo quadrática, pois isso garante que o problema seja de otimização convexa e será obtido uma solução analítica [47], como mostra o Apêndice A.

Outro fato interessante é que se os dados em $\mathbb{X}$ seguirem uma distribuição normal (multivariada), a estimação dos parâmetros pode ser interpretada como um problema de maximização de verossimilhanças, conceito fundamental em estatística $[2,10]$, que é uma das razões pela escolha de se trabalhar com modelos vetoriais autorregressivos.

Portanto, pelo Teorema da Representação de Mercer, pode-se mostrar que esse problema tem solução dada por

$$
f(\cdot)=\sum_{i=1}^{l} \alpha_{i} \kappa(\mathbf{x}(i), \cdot) \text {, para quaisquer } \alpha_{n} \text { em } \mathbb{R},
$$

4 Para uma versão mais geral do Teorema da Representação de Mercer, o leitor deve se referir a [46]. 
cujos coeficientes $^{5}$ da expansão são dados por,

$$
\boldsymbol{\alpha}=[\kappa(\mathbf{x}, \mathbf{x})+\lambda \mathbf{I}]^{-1} \mathbf{y}
$$

em que y é o vetor das variáveis dependentes do sistema em que se está trabalhando.

A próxima seção apresenta uma possível generalização dos resultados anteriores para o caso vetorial (multivariado).

\subsubsection{Reproducing kernel para funções vetoriais}

A primeira coisa a se notar é: todos os resultados vistos anteriormente têm um paralelo com o caso vetorial, de maior interesse aqui.

Como um todo, há duas diferenças:

(i) o reproducing kernel, agora, perfaz uma matriz, i.e., sendo os espaços de entrada e o de características, respectivamente, $\mathbb{X} \subset \mathbb{R}^{m}$ e $\mathbb{F} \subset \mathbb{R}^{D \times D}$, então,

$$
\begin{aligned}
\mathbf{K}: \mathbb{X} \times \mathbb{X} & \longrightarrow \mathbb{F} \\
(\mathbf{x}, \mathbf{y}) & \longmapsto \mathbf{K}(\mathbf{x}, \mathbf{y}) \succeq 0
\end{aligned}
$$

Um RKHS vetorial é um espaço de funções $\left(\mathbf{f}: \mathbb{X} \rightarrow \mathbb{R}^{D}\right)$ de Hilbert $\mathbb{F}=\mathbb{H}$, tal que para algum $\boldsymbol{\alpha}$ em $\mathbb{R}^{D}$ e $\mathbf{x}, \mathbf{K}(\mathbf{x}, \mathbf{y}) \boldsymbol{\alpha}$, como funções de $\mathbf{y}$, pertencem a $\mathbb{H}$. Além disso, se $\langle\cdot, \cdot\rangle$ é um produto interno em $\mathbb{H}$, então

$$
\langle\mathbf{f}(\cdot), \mathbf{K}(\cdot, \mathbf{x}) \boldsymbol{\alpha}\rangle=\mathbf{f}(\mathbf{x})^{\top} \boldsymbol{\alpha}
$$

e $\mathbf{K}(\cdot, \cdot)$ possui a propriedade reprodutiva. Note que (1.28) pode ser reescrito como

$$
\mathbf{f}(\mathbf{x})=\sum_{i=1}^{\infty} \mathbf{K}(\mathbf{x}(i), \mathbf{x}) \boldsymbol{\alpha}_{i}, \text { para quaisquer } \boldsymbol{\alpha}_{i} \text { em } \mathbb{R}^{D}
$$

em que, cada matriz $\mathbf{K}(\mathbf{x}(i), \mathbf{x})$ está operando sobre um vetor $\boldsymbol{\alpha}_{i}$.

(ii) A matriz de kernel agora possui estrutura bloco toeplitz, com elementos dados por $\mathbf{K}(\mathbf{X}, \mathbf{X})=[\mathbf{K}(\mathbf{x}(i), \mathbf{x}(j))]_{s, t}$, tais que, se $\mathbf{X}=\left\{\mathbf{x}_{i}(n)\right\}_{i=1}^{D}{ }_{n=1}^{N}$, a dimensão de $\mathbf{K}$ é $D N \times D N$.

Uma generalização da propriedade da aproximação universal para o caso vetorial também se aplica, ou seja, para o problema de minimização vetorial,

$$
\min _{\mathbf{f}(\mathbf{x}) \in \mathbb{H}}\{J(\mathbf{f}(\mathbf{x}))\}=\min _{\mathbf{f}(\mathbf{x}) \in \mathbb{H}}\left\{\sum_{j=1}^{D} \frac{1}{D} \sum_{i=1}^{N}\left(y_{j, i}-f_{j}\left(\mathbf{x}_{i}\right)\right)^{2}+\lambda\|\mathbf{f}(\mathbf{x})\|_{2}^{2}\right\},
$$

\footnotetext{
5 A dedução de como chegar nesse resultado é tratada no Apêndice A.
} 
$\operatorname{com} \mathbf{f}(\mathbf{x})=\left(f_{1}(\mathbf{x}), \cdots, f_{n}(\mathbf{x})\right)$, o Teorema da Representação de Mercer fornece,

$$
\mathbf{f}(\mathbf{x})=\sum_{i=1}^{\infty} \mathbf{K}(\mathbf{x}(i), \mathbf{x}) \boldsymbol{\alpha}_{i}, \text { para todo, } \boldsymbol{\alpha}_{i} \text { em } \mathbb{R}^{D}
$$

de modo que os coeficientes da expansão acima satisfazem $a^{6}$,

$$
\boldsymbol{\alpha}=[\mathbf{K}(\mathbf{X}, \mathbf{X})+\lambda N \mathbf{I}]^{-1} \mathbf{y}
$$

em que $\boldsymbol{\alpha}=\operatorname{vec} \boldsymbol{\alpha}_{i}, \boldsymbol{y}=\operatorname{vec} \boldsymbol{y}_{i}$ são vetores $(D N \times 1)$ e $\mathbf{K}(\mathbf{X}, \mathbf{X})=[\mathbf{K}(\mathbf{X}(i), \mathbf{X}(j))]_{s, t}$, para $i, j=1, \cdots, D$ e $s, t=1, \cdots, D$ é a matriz de Kernel toeplitz em blocos $(D N \times D N)$, ou seja,

$$
\mathbf{K}(\mathbf{X}, \mathbf{X})=\left[\begin{array}{ccc}
{[\mathbf{K}(\mathbf{X}(1), \mathbf{X}(1))]_{1,1}} & \cdots & {[\mathbf{K}(\mathbf{X}(1), \mathbf{X}(D))]_{1, D}} \\
\vdots & \ddots & \vdots \\
{[\mathbf{K}(\mathbf{X}(D), \mathbf{X}(1))]_{D, 1}} & \cdots & {[\mathbf{K}(\mathbf{X}(D), \mathbf{X}(D))]_{D, D}}
\end{array}\right]
$$

em que cada bloco $[\mathbf{K}(\mathbf{X}(i), \mathbf{X}(j))]_{s, t}$ é uma matriz $(N \times N)$ (note que foi feita a hipótese de que cada componente possui a mesma dimensão).

Na próxima seção são listados alguns dos kernels mais usados.

\subsubsection{Kernels mais usados}

Aqui, apresentam-se alguns dos kernels mais usados na literatura [16, 33, 36-38].

1. Kernels Projetivos

- Monomial

$$
\kappa_{\mathrm{M}}(\mathbf{x}, \mathbf{y})=\langle\mathbf{x}, \mathbf{y}\rangle^{d}
$$

- Polinomial

$$
\kappa_{\mathrm{P}}(\mathbf{x}, \mathbf{y})=(c+\langle\mathbf{x}, \mathbf{y}\rangle)^{d}
$$

- Exponencial

$$
\kappa_{\mathrm{E}}(\mathbf{x}, \mathbf{y})=\exp \left[\langle\mathbf{x}, \mathbf{y}\rangle /\left(2 \sigma^{2}\right)\right]
$$

- Sigmoidal

$$
\kappa_{\mathrm{S}}(\mathbf{x}, \mathbf{y})=\tanh [(\langle\mathbf{x}, \mathbf{y}\rangle / \sigma)+c]
$$

2. Kernels Radiais

- Laplaciano

$$
\kappa_{\mathrm{L}}(\mathbf{x}, \mathbf{y})=\exp \left[-\|\mathbf{x}-\mathbf{y}\|_{2} /\left(2 \sigma^{2}\right)\right]
$$

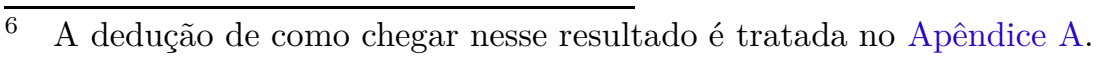


- Gaussiano

$$
\kappa_{\mathrm{G}}(\mathbf{x}, \mathbf{y})=\exp \left[-\|\mathbf{x}-\mathbf{y}\|_{2}^{2} /\left(2 \sigma^{2}\right)\right]
$$

- Multiquadratico

$$
\kappa_{\mathrm{MQ}}(\mathbf{x}, \mathbf{y})=\left(\|\mathbf{x}-\mathbf{y}\|_{2}^{2}+c\right)^{1 / 2}
$$

- Multiquadratico Inverso

$$
\kappa_{\mathrm{IMQ}}(\mathbf{x}, \mathbf{y})=\left(\|\mathbf{x}-\mathbf{y}\|_{2}^{2}+c\right)^{-1 / 2}
$$

- Racional

$$
\kappa_{\mathrm{R}}(\mathbf{x}, \mathbf{y})=1-\left[\|\mathbf{x}-\mathbf{y}\|_{2}^{2} /\left(\|\mathbf{x}-\mathbf{y}\|_{2}^{2}+c\right)\right]
$$

Os parâmetros $c$ e $\sigma$ são estritamente positivos e $p$ pertence a $\mathbb{N}^{*}$.

\subsection{ESCOLHA DO KERNEL}

Com uma escolha adequada do kernel os dados podem tornar-se separados no espaço de características, apesar de serem não-separáveis no espaço original. Cada escolha de kernel irá definir uma forma diferente de espaço de características, com seus respectivos vetores resultantes de características (também chamados de classificadores na literatura de Support Vector Machines), atuando sobre os dados de entrada.

Portanto, a escolha do kernel é um problema intrínseco e aberto nos métodos de kernel e, ainda, é necessário escolher parâmetros para os definirem matematicamente, como mostrado na Seção 1.1.7. Idealmente, o kernel deve conter o melhor "conhecimento", a priori, do problema em que se está trabalhando, para que possa refletir satisfatoriamente a dinâmica de um certo modelo. Por exemplo, se a densidade dos dados for uma questão relevante ao problema, uma boa escolha seriam os kernels radiais. Por outro lado, se o objetivo for realizar estimação de sinais, então um bom começo são os kernels projetivos.

\subsubsection{Criando kernels}

A construção de kernels é, também, um tema ativo e uma área de problema aberto em métodos de kernels. Há diversas técnicas para se elaborar um kernel positivo semidefinido e, até, aprendê-los através dos conjuntos de dados [33, 48-50].

Para exemplificar, considere dois kernels positivos semi-definidos $\kappa_{1}(\cdot, \cdot)$ e $\kappa_{2}(\cdot, \cdot)$. Pode-se provar que as seguintes regras, geram, de fato, outros kernels, a partir dos anteriores [33],

1. $\kappa(\mathbf{x}, \mathbf{y})=a \kappa_{1}(\mathbf{x}, \mathbf{y}), a \mathrm{em} \mathbb{R}^{+}$; 
2. $\kappa(\mathbf{x}, \mathbf{y})=\kappa_{1}(\mathbf{x}, \mathbf{y})+\kappa_{2}(\mathbf{x}, \mathbf{y}) ;$

3. $\kappa(\mathbf{x}, \mathbf{y})=\kappa_{1}(\mathbf{x}, \mathbf{y}) \kappa_{2}(\mathbf{x}, \mathbf{y})$;

4. $\kappa(\mathbf{x}, \mathbf{y})=\sum_{i=1}^{n} a_{i} \kappa_{1}(\mathbf{x}, \mathbf{y})+\sum_{j=1}^{m} b_{j} \kappa_{2}(\mathbf{x}, \mathbf{y}), a_{i}, b_{i}$ em $\mathbb{R}^{+} ;$

5. $\kappa(\mathbf{x}, \mathbf{y})=\exp \left(\kappa_{1}(\mathbf{x}, \mathbf{y})\right)$.

\subsection{SUMÁRIO}

Nesse capítulo, apresentou-se a teoria necessária para o desenvolver do restante desse texto. Os conceitos expostos aqui são usados como unidades elementares para as técnicas apresentadas no próximo capítulo.

Nesse texto, foca-se principalmente em aplicações de regressão para processamento de sinais. Com a ajuda do kernel trick, a regressão via kernel torna-se um procedimento natural para ajuste de modelos não-lineares e seus detalhes são apresentados no Apêndice A, no qual começa-se recordando regressão linear e, então, a regressão não-linear é discutida por meio de métodos de kernel.

Ainda, o autor desse texto reconhece que, na literatura atual, os métodos de kernels não possuem uma técnica concreta e adequada de escolha de kernels, parâmetros e também da seleção dos vetores necessários do espaço de características, tornando esses um problema aberto e necessário que se resolva, para aplicações concretas. 


\section{MÉTOdos DE KERNEL APLICADOS A MEDIDAS DE CAUSALI- DADE}

Neste capítulo são revistos conceitos ligados ao fluxo de informação, a partir da noção de causalidade, juntamente uma discussão sobre a kernelização dos métodos lineares largamente utilizados na caracterização da inferência da causalidade.

O capítulo termina com algumas considerações sobre as razões que levaram à escolha dos estimadores propostos.

\subsection{CAUSALIDADE E SUA INFERÊNICA}

Granger [51] propôs uma forma de causalidade que é relativamente simples de se operacionalizar com modelos lineares vetoriais autorregressivos (Vector Autoregressive - VAR). A idéia é a de que uma série temporal $x(n)$ Granger-causa outra $y(n)$ se o conhecimento passado da primeira melhora a previsibilidade da segunda série ${ }^{1}$. Assim,

$$
x(n) \underset{\text { Causa }}{\stackrel{\text { Granger }}{\longrightarrow}} y(n) .
$$

A causalidade de Granger possui a importante propriedade de ausência de simetria, i.e.,

$$
x(n) \underset{\text { Causa }}{\stackrel{\text { Granger }}{\longrightarrow}} y(n) \text { não implica } y(n) \underset{\text { Causa }}{\stackrel{\text { Granger }}{\longrightarrow}} x(n),
$$

fato que é distinto de outras formas de se caracterizar a relação entre séries temporais.

$\mathrm{Na}$ prática, a inferência da causalidade de Granger envolve o ajuste de modelos $\operatorname{VAR}(p)[10]$, da forma,

$$
\mathbf{x}(n)=\sum_{r=1}^{p} \mathbf{A}(r) \mathbf{x}(n-r)+\mathbf{w}(n)
$$

em que o processo $\{\mathbf{x}(n)\}_{n \in \mathbb{Z}}, D$-dimensional, é assumido de média nula, $\{\mathbf{w}(n)\}_{n \in \mathbb{Z}}$ é um processo de inovação, com estatística i.i.d.WN $\left(\mathbf{0}, \boldsymbol{\Sigma}_{\mathbf{w}}\right)$ e as matrizes $\mathbf{A}(r)$ são tais que,

$$
\mathbf{A}(r)=\left[\begin{array}{cccc}
a_{11}(r) & a_{12}(r) & \cdots & a_{1 D}(r) \\
a_{21}(r) & a_{22}(r) & \cdots & a_{2 D}(r) \\
\vdots & \vdots & \ddots & \vdots \\
a_{D 1}(r) & a_{D 2}(r) & \cdots & a_{D D}(r)
\end{array}\right]
$$

têm os coeficientes $a_{i j}(r)$ responsáveis por ditar a interação linear de $x_{j}(n-r)$ para $x_{i}(n)$. Portanto, a maneira mais simples de se inferir a causalidade da série $x_{j}(n)$ para $x_{i}(n)$ é testar estatisticamente a nulidade desses coeficientes, para todo $r$ (testes específicos, para a causalidade linear de Granger, podem ser apreciados em [10]).

\footnotetext{
1 A formalidade matemática pode ser apreciada em [10].
} 
A representação dessa idéia no domínio da frequência, possível devido ao uso intensivo dos modelos $\operatorname{VAR}(p)$, ganhou força com as proposições das Função de Transferência Direcionada [8] (Directed Transfer Function — DTF) e Coerência Parcial Direcionada [7] (Partial Directed Coherence — PDC), que são medidas complementares e duais de conectividade $[52,53]$.

Assim, quantificadores tais como a PDC recebem o nome de conectividade e a DTF, alcançablidade, pois,

1. a PDC permite a detecção de conexões diretas, já que ocorrem sem intervenção de estruturas;

2. a DTF permite a detecção de conexões indiretas, uma vez que há intervenção de estruturas.

Exemplos das afirmações 1 e 2 podem ser vistos em [52,53] e, também, serão tratados na Seção 3.3. Note que essas diferenças de interpretação permanecem válidas quando da generalização kernelizada considerada adiante.

Ainda, como há testes estatísticos para inferir a causalidade de Granger no domínio do Tempo, há formas adequadas relativas à DTF e PDC [22,23].

Na próxima seção será vista a kernelização dos modelos $\operatorname{VAR}(p)$, que objetiva obter o modelo $k \operatorname{VAR}(p)$ e, por consequência, também os descritores causais temporal e espectrais, no espaço de características.

\subsection{MODELO AUTORREGRESSIVO VETORIAL KERNELIZADO}

Nos últimos anos, os métodos de kernel ficaram em voga, motivando a busca modelo $\operatorname{VAR}(p)$ kernelizado e, assim, abrindo a possibilidade de se investigar interações não-lineares entre séries temporais. Como é explicado no Capítulo 1, se o processo de kernelização for feito com cuidado, as não-linearidades envolvidas, tornam-se (quasi-)lineares no espaço de características $\mathbb{F}$ e, então, pode-se aplicar um método linear nesse último espaço vetorial.

Dessa maneira, seja $\phi(\cdot)$ um mapeamento não-linear, induzido por um kernel de Mercer [54], do espaço de entrada $\mathbb{X}$, para o característico $\left(\phi: \mathbb{X} \subset \mathbb{R}^{N \times D} \rightarrow \mathbb{F}\right)$. A kernelização do modelo autorregressivo de ordem $p(\operatorname{VAR}(p))$ pode ser feita de maneira direta, i.e.,

$$
\boldsymbol{\phi}[\mathbf{x}(n)]=\sum_{r=1}^{p} \phi[\mathbf{x}(n-r)] \mathbf{A}^{\phi \mathbf{H}}(r)+\boldsymbol{\vartheta}^{\phi}(n),\left\{\boldsymbol{\vartheta}^{\phi}(n)\right\}_{n \in \mathbb{Z}} \sim \text { i.i.d.WN }\left(\mathbf{0}, \boldsymbol{\Sigma}_{\vartheta^{\phi}}\right) .
$$

A esse processo, aplica-se a terminologia processo Autorregressivo kernelizado Vetorial (kernel Vector Autoregressive - kVAR) [55, 56]. 
Aqui, a matriz $\mathbf{A}^{\phi}(r)$ mede a interação não-linear de $x_{j}(n-r)$ em $x_{i}(n)$, através dos coeficientes $a_{i j}^{\phi}(r)$.

Deve-se notar que o mapeamento não-linear, representado pela função $\phi(\cdot)$, necessita $\operatorname{preservar}^{2}$

$$
\mathbb{E}\left\{\boldsymbol{\phi}\left[\mathbf{x}_{i}(n)\right] \phi\left[\mathbf{x}_{i}(n-k)\right]\right\}=\mathbb{E}\left\{\kappa\left[\mathbf{x}_{i}(n), \mathbf{x}_{i}(n-k)\right]\right\},
$$

de modo que $\kappa(\cdot)$ seja kernel de Mercer. Isso garante estacionariedade fraca em ambos espaços de entrada $\mathbb{X}$ e de características $\mathbb{F}[16,54]$.

Porém, o mapeamento não-linear, realizado pela função $\phi$ não deve ser feito de forma direta, pois, como indicado anteriormente, a imagem do mapeamento $\phi$ (apontado acima por $\mathbb{F}$ ) possui dimensão muito maior do que de seu domínio $(\mathbb{X})$, dependo do kernel utilizado [33], o que torna infactível sua representação (computacionalmente).

Assim, como mostra (2.4) há uma maneira concisa e indireta se realizar o mapeamento, dando-se através do chamado 'kernel trick' [16,33], em que a estrutura de covariância canônica do sinal em estudo é substituída pelo cálculo de um kernel de Mercer (a ser escolhido), ou seja,

$$
\left[\Gamma_{i j}\right]=\left\langle\mathbf{x}_{i}(n), \mathbf{x}_{j}(n-k)\right\rangle \stackrel{\phi(\cdot)}{\Longrightarrow}\left[K_{i j}\right]=\left\langle\phi\left[\mathbf{x}_{i}(n)\right], \phi\left[\mathbf{x}_{j}(n-k)\right]\right\rangle
$$

em que os elementos da matriz de estrutura de covariância canônica são representados por $\left[\Gamma_{i j}\right]$ e os da kernelizada, $\left[K_{i j}\right]$, ou seja, após a aplicação do 'kernel trick'.

A seguir, vê-se como estimar os coeficientes de modelos $k \operatorname{VAR}(p)$.

\subsubsection{Estimando o processo autorregressivo kernelizado}

Assumindo que o vetor de séries $\{\mathbf{x}(n)\}_{n=1}^{N}$ (no espaço original $\mathbb{X}$ ) é conhecido, com todas as $N$ amostras para cada um dos $D$ processos e sempre com o mesmo período de amostragem. Além disso, seja a notação seguinte,

$$
\begin{aligned}
& \boldsymbol{\Phi}:=(\phi[\mathbf{x}(1)], \cdots, \phi[\mathbf{x}(N)]) \quad(D \times N), \quad \mathbf{A}^{\phi}:=\left(\mathbf{A}^{\phi}(1), \cdots, \mathbf{A}^{\phi}(p)\right) \quad(D \times D p), \\
& \mathbf{X}:=(\mathbf{X}(0), \cdots, \mathbf{X}(N-1)) \quad(D p \times N), \quad \boldsymbol{\Theta}^{\phi}:=\left(\boldsymbol{\vartheta}^{\phi}(1), \cdots, \boldsymbol{\vartheta}^{\phi}(N)\right) \quad(D \times N), \\
& \mathbf{X}(n):=\left[\begin{array}{c}
\phi[\mathbf{x}(n)] \\
\vdots \\
\phi[\mathbf{x}(n-p+1)]
\end{array}\right] \quad(D p \times 1), \quad \mathbf{J}_{i}:=\left[\begin{array}{c}
\mathbf{0}_{D(i-p-1) \times D p} \\
\mathbf{I}_{D p} \\
\mathbf{0}_{(N-D(i+1)) \times D p}
\end{array}\right] \quad(N \times D p), \\
& \begin{array}{rlrl}
\boldsymbol{\varphi} & :=\operatorname{vec}(\boldsymbol{\Phi}) & (D N \times 1), \quad \boldsymbol{\alpha}^{\phi}:=\operatorname{vec}\left(\mathbf{A}^{\phi}\right) & \left(D^{2} p \times 1\right), \\
\boldsymbol{\theta}^{\boldsymbol{\phi}}:=\operatorname{vec}\left(\boldsymbol{\Theta}^{\phi}\right) & (D N \times 1),
\end{array}
\end{aligned}
$$

em que o conhecido operador — vec denota empilhamento das colunas de uma matriz.

$2 \quad$ A prova de (2.4) encontra-se em [16] (Propriedade 11.7, p. 421-2). 
Portanto, usando as definições dadas em (2.6), o modelo $k \operatorname{VAR}(p)$ (2.3), para $n=1, \cdots, N$ e $i=p+1, \cdots, N$ pode ser compactamente reescrito como,

$$
\Phi=\mathbf{A}^{\phi}\left(\mathbf{J}_{i} \mathbf{X} \mathbf{J}_{i}\right)^{\top}+\Theta^{\phi} .
$$

Note que a matriz seletora $\mathbf{J}_{i}$ (uma janela deslizante), assim como em [36] (no caso univariado), ajudou na construção da forma compacta (2.7), na medida em que ela (como o próprio nome sugestiona) seleciona $p$ amostras consecutivas da matriz de dados $\mathbf{X}$, correspondente ao instante (discreto) de interesse.

Agora, pela aplicação do operador — vec a ambos os lados de (2.7), tem-se,

$$
\begin{aligned}
\operatorname{vec}(\boldsymbol{\Phi}) & =\operatorname{vec}\left(\mathbf{A}^{\phi}\left(\mathbf{J}_{i} \mathbf{X} \mathbf{J}_{i}\right)^{\top}\right)+\operatorname{vec}\left(\boldsymbol{\Theta}^{\phi}\right) \\
& =\left(\left(\mathbf{J}_{i} \mathbf{X} \mathbf{J}_{i}\right) \otimes \mathbf{I}_{D}\right) \operatorname{vec}\left(\mathbf{A}^{\phi}\right)+\operatorname{vec}\left(\boldsymbol{\Theta}^{\phi}\right)
\end{aligned}
$$

e, finalmente,

$$
\boldsymbol{\varphi}=\left(\left(\mathbf{J}_{i} \mathbf{X} \mathbf{J}_{i}\right) \otimes \mathbf{I}_{D}\right) \boldsymbol{\alpha}^{\phi}+\boldsymbol{\theta}^{\phi}
$$

que é a generalização, para o caso vetorial (ou multivariado), da abordagem realizada no caso de uma única variável em [36,37].

Observe que para o cálculo dos coeficientes $\boldsymbol{\alpha}^{\phi}$, pode-se usar o critério dos mínimos quadrados, porém no espaço de características, ou seja, com o uso da kernelização, o problema que poderia ter um critério de minimização complicado no espaço de entrada $\mathbb{X}$, passou a ser um problema de minimização convexa no espaço de características $\mathbb{F}$.

Logo, chamando o novo método de mínimos quadrados multivariado não-linearkernelizado (kernel-nonlinear-multivariate least-squares — knMVLS), a estimação de $\boldsymbol{\alpha}^{\phi}$ significa escolher o estimador que minimiza,

$$
\begin{aligned}
S\left(\boldsymbol{\alpha}^{\phi}\right) & =\sum_{i=p+1}^{N} \boldsymbol{\theta}_{i}^{\phi \top} \boldsymbol{\theta}_{i}^{\phi} \\
& =\sum_{i=p+1}^{N}\left(\boldsymbol{\varphi}_{i}-\left(\left(\mathbf{J}_{i} \mathbf{X} \mathbf{J}_{i}\right) \otimes \mathbf{I}_{D}\right) \boldsymbol{\alpha}^{\phi}\right)^{\top}\left(\boldsymbol{\varphi}_{i}-\left(\left(\mathbf{J}_{i} \mathbf{X} \mathbf{J}_{i}\right) \otimes \mathbf{I}_{D}\right) \boldsymbol{\alpha}^{\phi}\right)
\end{aligned}
$$

Assim, fazendo $\frac{\partial}{\partial \boldsymbol{\alpha}^{\phi}}\left[S\left(\boldsymbol{\alpha}^{\phi}\right)\right]=\mathbf{0}$, o estimador do $k n$ MVLS é

$$
\begin{aligned}
\widehat{\boldsymbol{\alpha}^{\phi}} & =\left(\sum_{i=p+1}^{N}\left(\mathbf{J}_{i} \mathbf{X} \mathbf{J}_{i}\right)^{\top} \mathbf{J}_{i} \mathbf{X} \mathbf{J}_{i}\right)^{-1}\left(\sum_{i=p+1}^{N}\left(\mathbf{J}_{i} \mathbf{X} \mathbf{J}_{i}\right)^{\top} \boldsymbol{\varphi}_{i}\right) \\
& \underbrace{=}\left(\sum_{i=p+1}^{N} \mathbf{J}_{i}^{\top} \mathbf{K} \mathbf{J}_{i}\right)^{-1}\left(\sum_{i=p+1}^{N}\left(\mathbf{J}_{i} \mathbf{X} \mathbf{J}_{i}\right)^{\top} \boldsymbol{\varphi}_{i}\right) \\
=\widehat{\mathbf{I}}^{-1} & \boldsymbol{\gamma}
\end{aligned}
$$

em que $\mathbf{K}=\mathbf{X}^{\top} \mathbf{X}$ é a matriz de Kernel em blocos. 
Note também, como é bem conhecido da teoria clássica de estimação [10,57], que os coeficientes ótimos são estimados via

$$
\widehat{\boldsymbol{\alpha}}=\widehat{\Gamma}^{-1} \widehat{\gamma}
$$

em que $\widehat{\Gamma}$ é a estimativa da estrutura de covariância dos dados e $\widehat{\gamma}$ é a estimativa da matriz de correlação-cruzada dos dados. Portanto, comparando (2.11) com (2.12), tem-se que, no caso kernelizado, as respectivas estimativas ficam escritas como,

$$
\widehat{\Gamma}=\sum_{i=p+1}^{N} \mathbf{J}_{i}^{\top} \mathbf{K} \mathbf{J}_{i}
$$

e

$$
\widehat{\gamma}=\sum_{i=p+1}^{N}\left(\mathbf{J}_{i} \mathbf{X} \mathbf{J}_{i}\right)^{\top} \boldsymbol{\varphi}_{i}
$$

com as definições de $\mathbf{J}_{i}$ e $\mathbf{K}$, dadas acima.

Na próxima seção discutem-se as propriedades assintóticas do estimador.

\subsubsection{Propriedades assintóticas dos mínimos quadrados kernelizado}

Lütkepohl [10] mostra que, sob certas hipóteses, as propriedades assintóticas para o estimador convencional (não-kernelizado) de mínimos quadrados multivariado (Multivariate Least Squares - MVLS) de um processo $D$-dimensional $\operatorname{VAR}(p)$ segue uma distribuição Gaussiana da forma: $\mathbf{N}_{D}(\mathbf{0}, \operatorname{Cov}[\operatorname{vec}(\widehat{\mathbf{A}})])$. Esse resultado pode ser estendido para o caso de modelos $k \operatorname{VAR}(p)$.

Hable [58] provou que métodos de kernel são assintoticamente normais com taxa $\sqrt{N}$ garantindo normalidade do estimador $k n \operatorname{MVLS}(2.11)$, que leva à

Proposição 1 (Propriedade assintótica dos mínimos quadrados kernelizado não-linear $(k n \mathrm{MVLS}))$. Seja $\{\mathbf{x}(n)\}$ um modelo $\mathrm{k} V A R(p)$ D-dimensional como em $(2.3)$ e $\widehat{\boldsymbol{\alpha}^{\phi}}=$ $\left(\sum_{i=p+1}^{N} \mathbf{J}_{i}^{\top} \mathbf{K} \mathbf{J}_{i}\right)^{-1}\left(\sum_{i=p+1}^{N} \mathbf{J}_{i}^{\top} \mathbf{K}^{i}\right)$ o estimador $\mathrm{kn} M V L S$. Então, $\operatorname{plim} \widehat{\boldsymbol{\alpha}^{\phi}}=\boldsymbol{\alpha}^{\phi}$

e

$\sqrt{N}\left(\widehat{\boldsymbol{\alpha}^{\phi}}-\boldsymbol{\alpha}^{\phi}\right) \stackrel{d}{\rightarrow} \mathrm{N}_{D}\left(\mathbf{0}, \Gamma^{-1} \boldsymbol{\Sigma}_{\vartheta^{\phi}}\right)$

em que

$\boldsymbol{\Gamma}:=\operatorname{plim}\left(\frac{1}{N} \sum_{i=p+1}^{N} \mathbf{J}_{i}^{\top} \mathbf{K} \mathbf{J}_{i}\right) \quad$ e $\quad \boldsymbol{\Sigma}_{\vartheta \phi}=\frac{1}{\sqrt{N}} \sum_{i=p+1}^{N}\left(\mathbf{J}_{i} \mathbf{X} \mathbf{J}_{i}\right)^{\top} \boldsymbol{\theta}_{i}^{\phi} \underset{N \rightarrow \infty}{\stackrel{d}{\longrightarrow}} \mathrm{N}_{D}\left(\mathbf{0}, \boldsymbol{\Gamma} \boldsymbol{\Sigma}_{\vartheta^{\phi}}\right)$. 
Para ser possível provar a proposição acima é necessário escrever (2.11) de outra forma, substituindo, ainda, $\boldsymbol{\varphi}_{i}$ por (2.9), ou seja,

$$
\begin{aligned}
\widehat{\boldsymbol{\alpha}^{\phi}} & =\left(\sum_{i=p+1}^{N} \mathbf{J}_{i}^{\top} \mathbf{K} \mathbf{J}_{i}\right)^{-1}\left(\sum_{i=p+1}^{N}\left(\mathbf{J}_{i} \mathbf{X} \mathbf{J}_{i}\right)^{\top}\left[\left(\left(\mathbf{J}_{i} \mathbf{X} \mathbf{J}_{i}\right) \otimes \mathbf{I}_{D}\right) \boldsymbol{\alpha}^{\phi}+\boldsymbol{\theta}_{i}^{\phi}\right]\right) \\
& =\boldsymbol{\alpha}^{\phi}+\left(\sum_{i=p+1}^{N} \mathbf{J}_{i}^{\top} \mathbf{K} \mathbf{J}_{i}\right)^{-1}\left(\sum_{i=p+1}^{N}\left(\mathbf{J}_{i} \mathbf{X} \mathbf{J}_{i}\right)^{\top} \boldsymbol{\theta}_{i}^{\phi}\right) \\
& =\boldsymbol{\alpha}^{\phi}+\widehat{\boldsymbol{\Gamma}}^{-1} \widehat{\boldsymbol{\Sigma}_{\vartheta^{\phi}}}
\end{aligned}
$$

Agora, tendo em vista as notações dadas em (2.6), sabe-se que (2.3) pode ser rescrito como,

$$
\boldsymbol{\phi}[\mathbf{x}(n)]=\mathbf{A}^{\phi}\left(\mathbf{J}_{i} \mathbf{X}(n-1) \mathbf{J}_{i}\right)^{\top}+\boldsymbol{\vartheta}^{\phi}(n) .
$$

Portanto, multiplicando à direita por $\left(\mathbf{J}_{i} \mathbf{X}(n-1) \mathbf{J}_{i}\right)$ e aplicando o operador esperança a ambos os lados de (2.16), chega-se à equações normais, que são similares, mas não idênticas às de Yule-Walker (como é explicado em [10], p. 72), ou seja,

$$
\mathbb{E}\left[\boldsymbol{\phi}[\mathbf{x}(n)]\left(\mathbf{J}_{i} \mathbf{X}(n-1) \mathbf{J}_{i}\right)\right]=\mathbf{A}^{\phi} \mathbb{E}\left[\left(\mathbf{J}_{i} \mathbf{X}(n-1) \mathbf{J}_{i}\right)^{\top}\left(\mathbf{J}_{i} \mathbf{X}(n-1) \mathbf{J}_{i}\right)\right]
$$

Agora, estimando $\mathbb{E}\left[\phi[\mathbf{x}(n)]\left(\mathbf{J}_{i} \mathbf{X}(n-1) \mathbf{J}_{i}\right)\right]$ por,

$$
\frac{1}{N}\left(\sum_{i=p+1}^{N} \boldsymbol{\Phi}_{i}\left(\mathbf{J}_{i} \mathbf{X} \mathbf{J}_{i}\right)\right)
$$

e $\mathbb{E}\left[\left(\mathbf{J}_{i} \mathbf{X}(n-1) \mathbf{J}_{i}\right)^{\top}\left(\mathbf{J}_{i} \mathbf{X}(n-1) \mathbf{J}_{i}\right)\right]$ como,

$$
\frac{1}{N}\left(\sum_{i=p+1}^{N} \mathbf{J}_{i}^{\top} \mathbf{K} \mathbf{J}_{i}\right),
$$

o estimador de $\mathbf{A}^{\phi}$ é dado por,

$$
\widehat{\mathbf{A}^{\phi}}=\left(\sum_{i=p+1}^{N} \boldsymbol{\Phi}_{i}\left(\mathbf{J}_{i} \mathbf{X} \mathbf{J}_{i}\right)\right)\left(\sum_{i=p+1}^{N} \mathbf{J}_{i}^{\top} \mathbf{K} \mathbf{J}_{i}\right)^{-1}
$$

Logo, substituindo (2.7) em (2.18), tem-se que,

$$
\begin{aligned}
\widehat{\mathbf{A}^{\phi}} & =\left(\sum_{i=p+1}^{N} \boldsymbol{\Phi}_{i}\left(\mathbf{J}_{i} \mathbf{X} \mathbf{J}_{i}\right)\right)\left(\sum_{i=p+1}^{N} \mathbf{J}_{i}^{\top} \mathbf{K} \mathbf{J}_{i}\right)^{-1} \\
& =\left(\sum_{i=p+1}^{N}\left(\mathbf{A}^{\phi}\left(\mathbf{J}_{i} \mathbf{X} \mathbf{J}_{i}\right)^{\top}+\boldsymbol{\Theta}_{i}^{\phi}\right)\left(\mathbf{J}_{i} \mathbf{X} \mathbf{J}_{i}\right)\right)\left(\sum_{i=p+1}^{N} \mathbf{J}_{i}^{\top} \mathbf{K} \mathbf{J}_{i}\right)^{-1} \\
& =\mathbf{A}^{\phi}+\left(\sum_{i=p+1}^{N} \boldsymbol{\Theta}_{i}^{\phi}\left(\mathbf{J}_{i} \mathbf{X} \mathbf{J}_{i}\right)\right)\left(\sum_{i=p+1}^{N} \mathbf{J}_{i}^{\top} \mathbf{K} \mathbf{J}_{i}\right)^{-1}
\end{aligned}
$$


Note que vec $\left(\widehat{\mathbf{A}^{\phi}}\right)=\widehat{\boldsymbol{\alpha}^{\phi}}$, como dado pelas notações em (2.6) e, finalmente, aplicando-se o operador convergência em probabilidade (lei fraca dos grandes números) a (2.19), vem que,

$$
\operatorname{plim}\left(\widehat{\mathbf{A}^{\phi}}-\mathbf{A}^{\phi}\right)=\operatorname{plim}\left(\frac{1}{N} \sum_{i=p+1}^{N} \boldsymbol{\Phi}_{i}\left(\mathbf{J}_{i} \mathbf{X} \mathbf{J}_{i}\right)\right) \operatorname{plim}\left(\frac{1}{N} \sum_{i=p+1}^{N} \mathbf{J}_{i}^{\top} \mathbf{K} \mathbf{J}_{i}\right)^{-1}=0
$$

pelo Lema 3.1 de [10] (p. 73, considerando-se, ainda, as devidas adaptações para o caso kernelizado) e levando em conta a última hipótese da Proposição 1, o que implica em $\operatorname{plim}\left(\frac{1}{N} \sum_{i=p+1}^{N} \boldsymbol{\Phi}_{i}\left(\mathbf{J}_{i} \mathbf{X} \mathbf{J}_{i}\right)\right)=0$. Logo, a consistência de $\widehat{\mathbf{A}^{\phi}}$ está estabelecida e, por consequência, a de $\widehat{\boldsymbol{\alpha}^{\phi}}$ também.

Agora, usando (2.15) vem que,

$$
\begin{aligned}
\sqrt{N}\left(\widehat{\boldsymbol{\alpha}^{\phi}}-\boldsymbol{\alpha}^{\phi}\right) & =\sqrt{N}\left(\sum_{i=p+1}^{N} \mathbf{J}_{i}^{\top} \mathbf{K} \mathbf{J}_{i}\right)^{-1}\left(\sum_{i=p+1}^{N}\left(\mathbf{J}_{i} \mathbf{X} \mathbf{J}_{i}\right)^{\top} \boldsymbol{\theta}_{i}^{\phi}\right) \\
& =\left(\frac{1}{N} \sum_{i=p+1}^{N} \mathbf{J}_{i}^{\top} \mathbf{K} \mathbf{J}_{i}\right)^{-1}\left(\frac{1}{\sqrt{N}} \sum_{i=p+1}^{N}\left(\mathbf{J}_{i} \mathbf{X} \mathbf{J}_{i}\right)^{\top} \boldsymbol{\theta}_{i}^{\phi}\right)
\end{aligned}
$$

que pela Proposição C.2(4), do Apêndice C de [10] (p. 683-4), $\sqrt{N}\left(\widehat{\boldsymbol{\alpha}^{\phi}}-\boldsymbol{\alpha}^{\phi}\right)$ possui a mesma distribuição assintótica de,

$$
\left[\operatorname{plim}\left(\frac{1}{N} \sum_{i=p+1}^{N} \mathbf{J}_{i}^{\top} \mathbf{K} \mathbf{J}_{i}\right)^{-1}\right]\left[\frac{1}{\sqrt{N}} \sum_{i=p+1}^{N}\left(\mathbf{J}_{i} \mathbf{X} \mathbf{J}_{i}\right)^{\top} \boldsymbol{\theta}_{i}^{\phi}\right]=\boldsymbol{\Gamma}^{-1} \boldsymbol{\Sigma}_{\boldsymbol{\vartheta}^{\phi}}
$$

Portanto, pelo Lema 3.1 de [10] (p. 73, considerando-se, ainda, as devidas adaptações para o caso kernelizado), a distribuição assintótica de $\sqrt{N}\left(\widehat{\boldsymbol{\alpha}^{\phi}}-\boldsymbol{\alpha}^{\phi}\right)$ é normal, com matriz de covariância dada por $\Gamma^{-1} \Sigma_{\vartheta^{\phi}}$.

Na próxima seção discutem-se a kernelização da seleção da ordem e a adequação do modelo.

\subsubsection{Seleção de ordem e adequação do modelo}

Como visto anteriormente, a estimativa dos coeficientes de um modelo $k \operatorname{VAR}(p)$ é não-viesada e assintoticamente normal, com estrutura de covariância dada por (2.13).

Agora, para se estimar a estrutura de covariância da inovação, do processo (kernelizado), pode-se recorrer à fórmula bem conhecida (refira-se a [10], p. 28-9, para a demonstração),

$$
\operatorname{vec} \boldsymbol{\Sigma}_{\vartheta^{\phi}}=\left(\mathbf{I}_{(D p)^{2}}-\mathcal{A}^{\phi} \otimes \mathcal{A}^{\phi}\right) \operatorname{vec} \Gamma(0)
$$


em que $\mathcal{A}^{\phi}$ é a matriz companheira controlável em blocos, da matriz de coeficientes $\mathrm{A}^{\phi}(r)$, para todo $r$, ou seja,

$$
\mathcal{A}^{\phi}=\left[\begin{array}{ccccc}
\mathbf{A}^{\phi}(1) & \mathbf{A}^{\phi}(2) & \cdots & \mathbf{A}^{\phi}(p-1) & \mathbf{A}^{\phi}(p) \\
\mathbf{I}_{D} & \mathbf{0}_{D \times D} & \cdots & \mathbf{0}_{D \times D} & \mathbf{0}_{D \times D} \\
\mathbf{0}_{D \times D} & \mathbf{I}_{D} & \cdots & \mathbf{0}_{D \times D} & \mathbf{0}_{D \times D} \\
\vdots & \vdots & \ddots & \vdots & \vdots \\
\mathbf{0}_{D \times D} & \mathbf{0}_{D \times D} & \cdots & \mathbf{I}_{D} & \mathbf{0}_{D \times D}
\end{array}\right]_{(D p \times D p)}
$$

Portanto, com essas informações em mãos, é possível definir o critérios de seleção de ordem do modelo e de checagem de sua adequação.

\subsubsection{Seleção da ordem do modelo}

Para escolha da ordem do modelo, generalizam-se os quatro critérios de informação contidos em [10], a saber,

1. Critério da Predição Final do Erro Kernelizado (Kernelized-Final Prediction Error $\left.-{ }_{k} \mathrm{FPE}\right)$

$$
{ }_{k} \mathrm{FPE}(m)=\left(\frac{N+D m+1}{N-D m-1}\right)^{D} \operatorname{det} \Sigma_{\vartheta^{\phi}} ;
$$

2. Critério de Informação de Akaike Kernelizado (Kernelized-Akaike Information Criterion $\left.-{ }_{k} \mathrm{AIC}\right)$

$$
{ }_{k} \mathrm{AIC}(m)=\ln \operatorname{det} \boldsymbol{\Sigma}_{\vartheta^{\phi}}+\frac{2 m D^{2}}{N}
$$

3. Critério de Hannan-Quinn Kernelizado (Kernelized-Hannan-Quinn Criterion $-{ }_{k} \mathrm{HQ}$ )

$$
{ }_{k} \mathrm{HQ}(m)=\ln \operatorname{det} \boldsymbol{\Sigma}_{\vartheta^{\phi}}+\frac{2 \ln \ln N}{N} 2 m D^{2} ;
$$

4. Critério (Bayesiano) de Schwarz Kernelizado (Kernelized-Schwarz (Bayesian) Criterion $\left.-{ }_{k} \mathrm{BIC}\right)$

$$
{ }_{k} \mathrm{BIC}(m)=\ln \operatorname{det} \boldsymbol{\Sigma}_{\vartheta^{\phi}}+\frac{2 \ln N}{N} 2 m D^{2}
$$

Agora, suponha que, sem perda de generalidade, $\operatorname{Cr}(m)=\ln \operatorname{det} \boldsymbol{\Sigma}_{\vartheta^{\phi}}+m c(N) / N$ represente uma das quatro funções acima. Portanto, dada uma ordem máxima $M \geq p$, a ordem estimada $\widehat{p}$ deve ser escolhida de forma a minimizar $\operatorname{Cr}(m)$, sobre $m=1, \cdots, M$ [10]. Matematicamente, tem-se que,

$$
\widehat{p}=\min _{m \in\{1, \cdots, M\}}\left\{\ln \operatorname{det} \Sigma_{\vartheta^{\phi}}+\frac{m c(N)}{N}\right\} .
$$


Como supõe-se que o estimador de $\boldsymbol{\Sigma}_{\vartheta^{\phi}}$ possui distribuição normal (vide Proposição 1), é possível transpor o resultado apresentado em [10] (Proposição 4.3, p. 151) aqui também, para se ter a

Proposição 2 (Comparação de pequena amostra do ${ }_{k} \mathrm{AIC},{ }_{k} \mathrm{HQ}$ e $\left.{ }_{k} \mathrm{BIC}\right)$. Seja $\boldsymbol{\phi}[\mathbf{x}(-M+$ $1)], \cdots, \boldsymbol{\phi}[\mathbf{x}(0)], \cdots, \boldsymbol{\phi}[\mathbf{x}(N)]]$ qualquer série temporal D-dimensional e suponha que modelos $\mathrm{k} \operatorname{VAR}(m), m=0, \cdots, M$ são ajustados a $\phi[\mathbf{x}(1), \cdots, \phi[\mathbf{x}(N)]]$. Então as seguintes relações valem, mesmo sem o pré-requisito de as séries serem estacionárias,

$$
\begin{aligned}
\widehat{p}\left({ }_{\mathrm{k}} B I C\right) & \leq \widehat{p}\left({ }_{\mathrm{k}} A I C\right) \quad \text { se } N \geq 8, \\
\widehat{p}\left({ }_{\mathrm{k}} B I C\right) & \leq \widehat{p}\left({ }_{\mathrm{k}} H Q\right) \quad \text { para todo } N, \\
\widehat{p}\left({ }_{\mathrm{k}} H Q\right) & \leq \widehat{p}\left({ }_{\mathrm{k}} A I C\right) \quad \text { se } N \geq 16,
\end{aligned}
$$

deixando estabelecido uma ordem de prioridade do uso dos critérios.

Para a prova da Proposição 2 o leitor deve referir-se a Seção 4.3.3 de [10], lembrando que a matriz de covariância do processo de inovações é para o caso kernelizado, ou seja, $\Sigma_{\vartheta^{\phi}}$, que possui um estimador consistente, como está descrito e formulado nas hipóteses da Proposição 1 (última equação).

\subsubsection{Adequação do modelo}

Para checar a adequação do modelo, realiza-se o teste dos resíduos, no espaço de características, usando o teste de Portmanteau refinado de Box-Ljung-Pierce [10], que tem por estatística,

$$
Q(h)=N^{2} \sum_{\tau=1}^{h} \frac{1}{N-\tau} \operatorname{tr}\left[\widehat{\boldsymbol{\Gamma}}(\tau)^{\top} \widehat{\boldsymbol{\Gamma}}(0)^{-1} \widehat{\boldsymbol{\Gamma}}(\tau) \widehat{\boldsymbol{\Gamma}}(0)^{-1}\right],
$$

em que $\boldsymbol{\Gamma}$ é estimado via (2.13).

Sob a hipótese nula,

$$
\mathcal{H}_{0} \text { : os resíduos do ajuste (2.3) são brancos, }
$$

a estatística (2.31) possui distribuição chi-quadrada, com $D^{2}(h-p)$ graus de liberdade, ou seja, $Q(h) \stackrel{d}{\rightarrow} \chi_{\nu}^{2}$, tal que $\nu=D^{2}(h-p)$.

A próxima seção expõe como obter a fatoração espectral, no caso do modelo $k \operatorname{VAR}(p)$.

\subsection{PSEUDO-DENSIDADE ESPECTRAL}

Como se sabe, na análise espectral vetorial, objeto dessa tese, quer-se estimar a matriz densidade espectral, a partir dos dados. Portanto, é natural querer transpor o que se tem do conhecido caso linear [59], para um caso mais geral, ou seja, kernelizado. 
Logo, nesta seção explora-se a kernelização da matriz densidade espectral.

\subsubsection{Matriz densidade espectral kernelizada}

Em analogia com o modelo linear $\operatorname{VAR}(p)$ (ver [59], cap. 15), o modelo $k \operatorname{VAR}(p)$ em (2.3) possui transformada $z$ dada por,

$$
\boldsymbol{\Phi}[\mathbf{X}(z)]=\sum_{r=1}^{p} \boldsymbol{\Phi}[\mathbf{X}(z)] \mathbf{A}^{\phi \mathrm{H}}(r) z^{-r}+\mathbf{\Theta}^{\phi}(z)
$$

e, portanto, chamando $\overline{\mathbf{A}}^{\phi}(z)=\mathbf{I}-\sum_{r=1}^{p} \mathbf{A}^{\phi}(r) z^{-r}$, tem-se,

$$
\boldsymbol{\Phi}[\mathbf{X}(z)]=\Theta^{\phi}(z)\left\{\left[\overline{\mathbf{A}}^{\phi}(z)\right]^{-1}\right\}^{\mathrm{H}}
$$

$\mathrm{e}$

$$
\boldsymbol{\Phi}[\mathbf{X}(z)]^{\mathrm{H}}=\left[\overline{\mathbf{A}}^{\phi}(z)\right]^{-1} \boldsymbol{\Theta}^{\phi}(z)^{\mathrm{H}},
$$

em que $z=\mathrm{e}^{-\mathbf{i} 2 \pi f}$ e $\mathbf{i}^{2}=-1$.

Do Teorema de Wiener-Khintchine [60], sabe-se que, para modelos lineares a densidade espectral é escrita como $\mathbf{S}=\mathcal{F}\left[\mathbb{E}\left[\mathbf{x}(n) \mathbf{x}(n)^{\mathrm{H}}\right]\right]=\mathcal{F}[\boldsymbol{\Gamma}(\tau)][59]$, sendo que $\boldsymbol{\Gamma}(\tau)$ é a estrutura de covariância dos dados, $\mathbf{x}^{\mathrm{H}}$ significa a operação de transposição conjugada (Hermitiano) do vetor $\mathbf{x}$ e o operador $\mathcal{F}[\cdot]$ é a transformada de Fourier. Logo,

$$
\begin{aligned}
\mathbf{S}_{k \operatorname{VAR}(p)}(f) & =\mathcal{F}[\boldsymbol{\Gamma}(\tau)] \\
& =\mathcal{F}[\mathbb{E}\{\kappa(\mathbf{x}(n), \mathbf{x}(n))\}] \\
& =\mathcal{F}[\mathbb{E}\{\langle\boldsymbol{\phi}[\mathbf{x}(n)], \phi[\mathbf{x}(n)]\rangle\}] \\
& =\mathcal{F}\left[\mathbb{E}\left\{\boldsymbol{\phi}[\mathbf{x}(n)]^{\mathrm{H}} \boldsymbol{\phi}[\mathbf{x}(n)]\right\}\right] \\
& =\boldsymbol{\Phi}[\mathbf{X}(f)]^{\mathrm{H}} \mathbf{\Phi}[\mathbf{X}(f)]
\end{aligned}
$$

Agora, fazendo $z=\mathrm{e}^{-\mathbf{i} 2 \pi f}$ em (2.33) e (2.34) e substituindo em (2.35), vem que,

$$
\mathbf{S}_{k \operatorname{VAR}(p)}(f)=\left[\overline{\mathbf{A}}^{\phi}(f)\right]^{-1} \boldsymbol{\Theta}^{\phi}(f)^{\mathrm{H}} \boldsymbol{\Theta}^{\phi}(f)\left\{\left[\overline{\mathbf{A}}^{\phi}(f)\right]^{-1}\right\}^{\mathrm{H}}
$$

Finalmente, como $\boldsymbol{\Theta}^{\phi}(f)^{\mathrm{H}} \boldsymbol{\Theta}^{\phi}(f)=\boldsymbol{\Sigma}_{\vartheta^{\phi}}$ (vide modelo $k \operatorname{VAR}(p)(2.3)$ ), tem-se,

$$
\mathbf{S}_{k \operatorname{VAR}(p)}(f)=\left[\overline{\mathbf{A}}^{\phi}(f)\right]^{-1} \boldsymbol{\Sigma}_{\vartheta^{\phi}}\left\{\left[\overline{\mathbf{A}}^{\phi}(f)\right]^{-1}\right\}^{\mathrm{H}},
$$

que pode ser vista como a matriz da pseudo-densidade espectral.

Portanto, com essa definição canônica e kernelizada da fatoração da pseudo-matriz densidade espectral, tem-se o ponto de partida dos descritores de conectividade, que serão vistos na próxima seção, assim como suas propriedades assintóticas. 


\subsection{DESCRITORES DE CONECTIVIDADE KERNELIZADOS}

Uma pesquisa recente sobre os testes estatísticos para caracterizar a causalidade de Granger não-linear [17, 26-31,61,62], em ambos domínios do tempo e frequência, chama a atenção de que é possível ter essas duas formas de representação da estrutura de covariância, dando lugar a quantificadores que são introduzidos aqui: o teste da causalidade de Granger-não-linear-kernelizada (kernel-nonlinear-Granger Causality Test knGCT), Função de Transferência Direcionada-não-linear-kernelizada (kernel-nonlinearDirected Transfer Function - knDTF) e Coerência Parcial Direcionada-não-linear-kernelizada (kernel-nonlinear-Partial Directed Coherence - knPDC) $[55,56]$.

Aqui, exploram-se algumas de suas características, tais como equações para seus cálculos e propriedades assintóticas aliadas.

\subsubsection{Domínio do tempo}

Com a finalidade de testar a conectividade, usando um modelo ajustado de (2.3), através de uma versão kernelizada da causalidade de Granger não-linear ( $k n \mathrm{GCT}$ ), basta examinar a nulidade $\operatorname{dos} a_{i j}^{\phi}(r)$, aplicando uma estatística de Wald modificada [10].

Pode-se formular o teste como,

$$
\mathcal{H}_{0}: \mathbf{C} \boldsymbol{\alpha}^{\phi}=\mathbf{0} \quad \text { vs. } \quad \mathcal{H}_{1}: \mathbf{C} \boldsymbol{\alpha}^{\phi} \neq \mathbf{0}
$$

em que $\mathbf{C}$ é uma matriz de seleção estrutural, tal que para a hipótese nula $\mathcal{H}_{0}$ seja possível capturar $a_{i j}^{\phi}(r)=0$ para todo $r$. Com base na Proposição 1, pode-se escrever,

$$
\sqrt{N}\left(\widehat{\mathbf{C} \boldsymbol{\alpha}^{\phi}}-\mathbf{C} \boldsymbol{\alpha}^{\phi}\right) \stackrel{d}{\rightarrow} \mathrm{N}_{D}\left(\mathbf{0}, \mathbf{C}\left(\boldsymbol{\Gamma}^{-1} \boldsymbol{\Sigma}_{\vartheta^{\phi}}\right) \mathbf{C}^{\top}\right)
$$

e a estatística kernelizada de Wald fica dada por,

$$
N{\widehat{\boldsymbol{\alpha}^{\phi}}}^{\top} \mathbf{C}^{\top}\left[\mathbf{C}\left(\boldsymbol{\Gamma}^{-1} \boldsymbol{\Sigma}_{\vartheta^{\phi}}\right) \mathbf{C}^{\top}\right]^{-1} \widehat{\mathbf{C} \boldsymbol{\alpha}^{\phi}} \stackrel{d}{\rightarrow} \chi_{\nu}^{2}, \nu=\operatorname{posto} \mathbf{C} .
$$

Usando os estimadores de kernel (2.13) e (2.23), respectivamente, para $\boldsymbol{\Gamma}$ e $\boldsymbol{\Sigma}_{\vartheta^{\phi}}$, a estatística resultante é

$$
{ }_{\kappa} \lambda_{W}={\widehat{\boldsymbol{\alpha}^{\phi}}}^{\top} \mathbf{C}^{\top}\left\{\mathbf{C}\left[\left(\sum_{i=p+1}^{N} \mathbf{J}_{i}^{\top} \mathbf{K} \mathbf{J}_{i}\right)^{-1} \widehat{\boldsymbol{\Sigma}}_{\vartheta^{\phi}}\right] \mathbf{C}^{\top}\right\}^{-1} \widehat{\mathbf{C}} \widehat{\boldsymbol{\alpha}^{\phi}}
$$

em que ${ }_{\kappa} \lambda_{W} \stackrel{d}{\rightarrow} \chi_{\nu}^{2}$ (com $\nu=$ posto $\left.\mathbf{C}\right)$, dado que $\left\{\mathbf{x}_{i}(n)\right\}_{i=1}^{D} \stackrel{N=1}{N}$ concorda com as condições da Proposição 1. Ainda, devido a essas restrições,

$$
\left\{\mathbf{C}\left[\left(\sum_{i=p+1}^{N} \mathbf{J}_{i}^{\top} \mathbf{K} \mathbf{J}_{i}\right)^{-1} \widehat{\Sigma}_{\vartheta^{\phi}}\right] \mathbf{C}^{\top}\right\}^{-1} / N
$$

é um estimador consistente de $\left[\mathbf{C}\left(\boldsymbol{\Gamma}^{-1} \widehat{\boldsymbol{\Sigma}}_{\vartheta^{\phi}}\right) \mathbf{C}^{\top}\right]^{-1} / N$. Portanto, tem-se a seguinte 
Proposição 3 (Propriedades assintóticas da estatística de Wald kernelizada). Com base na Proposição 1 e sabendo que sabendo que $\operatorname{plim}\left(\frac{1}{N} \sum_{i=p+1}^{N} \mathbf{J}_{i}^{\top} \mathbf{K} \mathbf{J}_{i}\right)=\mathbf{\Gamma} \succ 0$, plim $\left(\widehat{\boldsymbol{\Sigma}}_{\vartheta^{\phi}}\right)=$ $\boldsymbol{\Sigma}_{\vartheta^{\phi}} \succ 0$ e $\mathcal{H}_{0}: \mathbf{C} \boldsymbol{\alpha}^{\phi}=\mathbf{0}_{M \times 1}$ são verdadeiros, com $\mathbf{C}$ uma matriz $\left(M \times\left(D^{2} p+D\right)\right)$ de posto $M$, então,

$$
{ }_{\kappa} \lambda_{W}={\widehat{\boldsymbol{\alpha}^{\phi}}}^{\top} \mathbf{C}^{\top}\left\{\mathbf{C}\left[\left(\sum_{i=p+1}^{N} \mathbf{J}_{i}^{\top} \mathbf{K} \mathbf{J}_{i}\right)^{-1} \widehat{\boldsymbol{\Sigma}}_{\vartheta^{\phi}}\right] \mathbf{C}^{\top}\right\}^{-1} \widehat{\mathbf{C} \boldsymbol{\alpha}^{\phi}} \stackrel{d}{\rightarrow} \chi_{\nu}^{2}, \nu=\text { posto } \mathbf{C} .
$$

Para a prova, o leitor deve referir-se à Proposição 3.5 e Apêndice C.7 de [10] (p. 103 e 694, respectivamente), lembrando que estrutura de covariância no caso kernelizado é dada por (2.13).

\subsubsection{Domínio da frequência}

Para se conseguir uma descrição kernelizada no domínio da frequência, basta empregar $\mathbf{A}^{\phi}(r)$ nas definições da Coerência Parcial Direcionada (PDC) [63, 64], i.e.,

$$
\bar{A}_{i j}^{\phi}(f)=\delta_{i j}-\sum_{r=1}^{p} a_{i j}^{\phi}(r) \mathrm{e}^{-\mathbf{i} 2 \pi f r},\left(\mathbf{i}^{2}=-1\right)
$$

em que as colunas são indicadas por $\overline{\mathbf{a}}_{j}^{\phi}(f)$, ou seja, $\overline{\mathbf{A}}^{\phi}(f)=\left[\overline{\mathbf{a}}_{1}^{\phi}(f) \cdots \overline{\mathbf{a}}_{j}^{\phi}(f)\right]$. De (2.43) também é possível definir a matriz função de transferência, ou seja,

$$
\mathbf{H}^{\phi}(f)=\left[\overline{\mathbf{A}}^{\phi}(f)\right]^{-1}
$$

com suas entradas e linhas denotadas, respectivamente, por $H_{i j}^{\phi}(f)$ e $\mathbf{h}_{i}^{\phi}(f)\left(\mathbf{H}^{\phi}(f)=\right.$ $\left[H_{i j}^{\phi}(f)\right]$ e $\overline{\mathbf{H}}^{\phi}(f)=\left[\overline{\mathbf{h}}_{1}^{\phi}(f) \cdots \overline{\mathbf{h}}_{i}^{\phi}(f)\right]^{\top}$ ), de forma que é possível kernelizar a Função de Transferência Direcionada (DTF) [8,22]. Aqui, denotam-se, as versões kernelizadas da PDC e DTF respectivamente, por $k n \mathrm{DTF}$ e $k n \mathrm{PDC}$.

Portanto, da argumentação acima, a knDTF é dada por,

$$
\kappa \eta \gamma_{i j}(f)=\frac{H_{i j}^{\phi}(f) \sqrt{\sigma_{i i}^{\phi}}}{\sqrt{\mathbf{h}_{i}^{\phi \mathrm{H}}(f) \Sigma_{\vartheta^{\phi}} \mathbf{h}_{i}^{\phi}(f)}}
$$

e a $k n \mathrm{PDC}$,

$$
{ }_{\kappa \eta} \pi_{i j}(f)=\frac{\bar{A}_{i j}^{\phi}(f) / \sqrt{\sigma_{i i}^{\phi}}}{\sqrt{\overline{\mathbf{a}}_{j}^{\phi \mathrm{H}}(f) \Sigma_{\vartheta^{\phi}}^{-1} \overline{\mathbf{a}}_{j}^{\phi}(f)}}
$$

As quantidades (2.45) e (2.46) são complementares e duais $[52,53]$ e têm valor igual, quando $D=2(i \neq j)$. Por outro lado, Quando $D>2$ a $k n \mathrm{PDC}$ tende a expor ligações diretas entre as séries, enquanto a $k n \mathrm{DTF}$ tende a exibir, também, conexões indiretas entre as séries. 
Aqui, novamente, invocando a Proposição 1 (devido ao resultado provado por [58]), transpõem-se os resultados assintóticos derivados para a DTF [22] e PDC [23], de forma direta, para a $k n \mathrm{DTF}$ e $k n \mathrm{PDC}$, em termos do kernel trick.

Assim, deve-se ter a

Proposição 4 (Propriedades assintóticas da Função de Transferência Direcionada-não-linear-kernelizada). Estendendo os resultados apresentados em [22], as propriedades assintóticas da $\mathrm{kn} D T F$ são,

1. Para $N \rightarrow \infty$, normalidade assintótica é garantida para (2.45),

$$
\sqrt{N}\left[\left|\kappa \widehat{\gamma_{i j}(f)}\right|^{2}-\left|\kappa \eta \gamma_{i j}(f)\right|^{2}\right] \stackrel{d}{\rightarrow} \mathrm{N}\left(0, \gamma^{2}(f)\right)
$$

2. Sob a hipótese nula,

$$
\mathcal{H}_{0}:\left|{ }_{\kappa \eta} \gamma_{i j}(f)\right|^{2}=0
$$

e sob certas condições para $\gamma^{2}(f)$ (referir-se a [22], para as condições), a distribuição resultante tem a forma,

$$
N\left[\mathbf{h}_{i}^{\phi \mathrm{H}}(f) \boldsymbol{\Sigma}_{\vartheta^{\phi}} \mathbf{h}_{i}^{\phi}(f)\right]\left[\left|\widehat{\kappa \eta} \gamma_{i j}(f)\right|^{2}-\left|{ }_{\kappa \eta} \gamma_{i j}(f)\right|^{2}\right] \stackrel{d}{\rightarrow} \sum_{k=1}^{q} l_{k}(f) \chi_{1}^{2},
$$

em que detalhes sobre $l_{k}(f)$ podem ser apreciadas em [22] e em suas referências contidas.

e a

Proposição 5 (Propriedades assintóticas da Coerência Parcial Direcionada-não-linear-kernelizada). Estendendo os resultados apresentados em [23], as propriedades assintóticas da $\mathrm{kn} P D C$ são,

1. Para $N \rightarrow \infty$, normalidade assintótica é garantida para (2.46),

$$
\sqrt{N}\left[\left|\kappa \overline{\pi_{i j}(f)}\right|^{2}-\left|\kappa \eta \pi_{i j}(f)\right|^{2}\right] \stackrel{d}{\rightarrow} \mathrm{N}\left(0, \gamma^{2}(f)\right) ;
$$

2. Sob a hipótese nula,

$$
\mathcal{H}_{0}:\left|{ }_{\kappa \eta} \pi_{i j}(f)\right|^{2}=0
$$

e sob certas condições para $\gamma^{2}(f)$ (referir-se a [23], para as condições), a distribuição resultante tem a forma,

$$
N\left[\overline{\mathbf{a}}_{j}^{\phi \mathbf{H}}(f) \boldsymbol{\Sigma}_{\vartheta^{\phi}}^{-1} \overline{\mathbf{a}}_{j}^{\phi}(f)\right]\left[\left|\widehat{\kappa \eta} \widehat{\pi_{i j}(f)}\right|^{2}-\left|{ }_{\kappa \eta} \pi_{i j}(f)\right|^{2}\right] \stackrel{d}{\rightarrow} \sum_{k=1}^{q} l_{k}(f) \chi_{1}^{2},
$$

em que detalhes sobre $l_{k}(f)$ podem ser apreciadas em [23] e em suas referências contidas. 
Para a prova da Proposição 4, o leitor deve referir-se à Seção 3.2 de [22] e para a Proposição 5, à Seção 3 de [23], sempre usando o fato de que a estrutura de covariância no caso kernelizado é dada por (2.13).

\subsection{SUMÁRIO}

Nesse Capítulo foram propostos alguns novos estimadores de causalidade de Granger para ambos domínios do tempo e frequência, assim como para a matriz densidade espectral, apenas baseado na kernelização do modelo linear $\operatorname{VAR}(p)$. Note que isso só foi possível graças ao kernel trick, que dispensou o mapeamento explícito das séries temporais para outro espaço de dimensão maior.

O próximo capítulo, contem alguns exemplos ilustrativos dessas técnicas, confirmando o êxito da teoria proposta. 


\section{ILUSTRAÇÕES NUMÉRICAS}

O objetivo do presente capítulo é mostrar a concreta oportunidade de uso das técnicas kernelizadas, como as introduzidas no Capítulo 2 e, também, ilustrar como inferir o fluxo de informação entre séries temporais.

Assim, analisam-se exemplos representativos de mapas lineares e não-lineares, da literatura. Isso permite oferecer uma perspectiva da capacidade de aplicações dos métodos kernelizados de inferência de causalidade.

Por fim, verifica-se que utilizando a estrutura de covariância kernelizada, ou seja, com a aplicação do kernel trick, infere-se corretamente a direção de interação entre as séries, desde que se faça uma escolha apropriada do kernel de Mercer para o modelamento das séries no espaço de características.

\subsection{MÉTODO}

O método proposto é composto de duas fases: (a) mapeamento (implícito) pelo kernel trick das séries e, (b) fatoração espectral da matriz de covariância kernelizada, tanto no domínio do tempo, quanto no da frequência, respectivamente, para a $k n \mathrm{GCT}$ e ambas $k n \mathrm{PDC}$ e $k n \mathrm{DTF}$.

\subsubsection{Mapeamento (implícito)}

Seja $\mathbf{x}(n)$ um vetor de séries temporais $D$-dimensional, em que cada componente possui $N$ amostras e $n$ representa o indexador de tempo discreto. Como foi discutido no Capítulo 1, associada a $\mathbf{x}(n)$, tem-se $\phi[\mathbf{x}(n)]$, resultante da kernelização, via o mapeamento,

$$
\begin{aligned}
\phi: \mathbb{X} & \longrightarrow \mathbb{F} \\
\mathbf{x}(n) & \longmapsto \phi[\mathbf{x}(n)],
\end{aligned}
$$

refletindo uma medida da não-linearidade do espaço de entrada.

Assim, é possível testar as relações causais entre as séries no espaço de características, que foram, de certa forma, linearizadas, a partir de modelamento linear autorregressivo, como discutido no Capítulo 2.

Note que essa proposta é mais geral do que aquela discutida em [13], ou seja, via entropia deslizante. Isso é devido ao que neste último caso faz-se um mapeamento, de forma direta, da medida de complexidade da série original, na tentativa de se obter uma descrição causal das séries mapeadas, sem garantia de que relações lineares sejam preservadas. Porém, no primeiro caso garante-se a Gaussianidade, ainda que assintótica 
[58], dos estimadores e linearidade das séries mapeadas, tornando consistente os testes estatísticos adotados.

\subsubsection{Análise de causalidade}

A segunda etapa do método consiste em analisar as séries mapeadas através das técnicas kernelizadas de causalidade de Granger, no domínio do tempo e da frequência.

Para tanto, utilizam-se, para o domínio do tempo, a $k n$ GCT e na frequência, as $k n$ DTF e knPDC, como vistos no Capítulo 2.

\subsection{CONVENÇÕES PARA APRESENTAR A $(K N) \mathrm{DTF} /(K N) \mathrm{PDC}$}

A convenção matricial usual de se apresentar os descritores causais no domínio da frequência [7] é adotada.

Assim, no caso do domínio da frequência, os gráficos colados na diagonal principal representam o (pseudo-)espectro (kernelizado e em unidades arbitrárias normalizadas, mas na escala logarítmica) e os que estão fora da diagonal retratam o conteúdo espectral de fluxo de informação na direção $\boldsymbol{i} \leftarrow \boldsymbol{j}$, tanto no caso do $(k n) \mathrm{PDC}$, como no do $(k n) \mathrm{DTF}$.

As cores nos gráficos, são, respectivamente,

- traçado em preto: (pseudo-)densidade espectral das séries;

- traçado em vermelho: valores estatisticamente significantes da $(k n) \mathrm{DTF} /(k n) \mathrm{PDC}$;

- traçado descontínuo em preto: limiar da aproximação de Patnaik [23], com nível de significância igual a $\alpha$;

- traçado em verde: valores estatisticamente não-significantes da $(k n) \mathrm{DTF} /(k n) \mathrm{PDC}$;

- pontilhados em preto: indicam os limites superior e inferior do intervalo de confiança da $(k n) \mathrm{DTF} /(k n) \mathrm{PDC}$, a $(1-\alpha) \times 100 \%[22,23]$.

Convenções análogas são aplicadas aos demais gráficos apresentados nesse Capítulo.

\subsection{VALIDAÇÃO ESTATÍSTICA DO MÉTODO PROPOSTO}

O objetivo desta seção é ilustrar o desempenho estatístico do método proposto. Para tanto, serão mostrados alguns exemplos de simulações de Monte Carlo de dados sintéticos, confrontando o comportamento dos métodos lineares com os não-lineares, propostos nesse texto. Assim, selecionaram-se quatro modelos, a saber, 
1. modelo autorregressivo de ordem um $(\operatorname{VAR}(1)) 3 \mathrm{D}$ [7];

2. modelo não-linear de banda limitada 2D [14,15];

3. modelo não-linear de banda larga 2D [65];

4. modelo não-linear de banda larga 3D [66].

Os resultados apresentados doravante, contam com o fato de que os dados foram kernelizados com sucesso e, ainda, houve um bom ajuste do modelo $k \operatorname{VAR}(p)$.

Quanto às simulações, em todos os casos, comprimentos $N$ das realizações podem variar no conjunto $\{256,512,1024\}$. A fim de evitar efeitos do transitório, desconsideramse os 10.000 primeiros pontos de cada série. As tabelas de contingência, que serão mostradas a seguir, sempre se referem a detalhes do procedimento de detecção estatística, dos métodos explorados nesse texto, em função do comprimento $N$ das séries e, do fator de acoplamento $c$, entre as séries.

Nos exemplos que se seguem, adota-se o seguinte protocolo de exposição,

(i) figuras de uma realização típica, simulada com $N=1024$ e um fator de acoplamento $c$ entre as séries, serão apresentadas;

(ii) figuras da $i \mathrm{PDC}$ (PDC-informacional) [64] e da iDTF (DTF-informacional) [22], baseadas no modelamento linear da realização, no espaço de entrada das séries serão, também, mostradas com o intuito de esclarecer que essas técnicas lineares têm dificuldade de capturar a correta direção de causalidade;

e, finalmente,

(iii) figuras e tabelas de contingência do $k n \mathrm{GCT}, k n \mathrm{PDC}$ e do $k n \mathrm{DTF}$, expondo a habilidade desses novos descritores de causalidade em aferir o sentido do fluxo de informação.

Aqui nada se diz a respeito do comportamento da GCT ordinária, pois em [67] foi mostrado (experimentalmente) que ambas GCT e $i \mathrm{PDC}$ são estimadores equivalentes da causalidade de Granger.

Vale observar que as tabelas de contingência representam porcentagens, de verdadeiros positivos (VP) e falsos positivo (FP), obtidas a partir de 10.000 replicações de Monte Carlo. 
3.3.1 Exemplo 1: Modelo autorregressivo de ordem um (VAR(1)) 3D

A fim de se comparar o comportamento das técnicas puramente lineares, com as kernelizadas, analisou-se o seguinte modelo $\operatorname{VAR}(1)$ de três variáveis, retirado de [7], ou seja,

$$
\left[\begin{array}{l}
x_{1}(n) \\
x_{2}(n) \\
x_{3}(n)
\end{array}\right]=\left[\begin{array}{rrr}
0,5 & 0,3 & 0,4 \\
-0,5 & 0,3 & 1,0 \\
0,0 & -0,3 & -0,2
\end{array}\right]\left[\begin{array}{l}
x_{1}(n-1) \\
x_{2}(n-1) \\
x_{3}(n-1)
\end{array}\right]+\left[\begin{array}{l}
w_{1}(n) \\
w_{2}(n) \\
w_{3}(n)
\end{array}\right]
$$

em que $\left\{w_{i}(n)\right\}_{n \in \mathbb{Z}}$ são processos de inovações i.i.d. normais, padrões.

É imediato, apenas observando as equações de (3.2), que se tenha o seguinte diagrama direcionado de conectividade, dado pela Figura 2.

Figura 2 - Padrão de conectividade do exemplo 1.

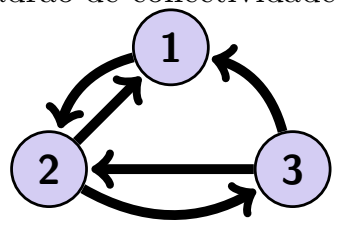

Fonte: produção do próprio autor.

Por sua vez, é fácil ver, na Figura 3, que o diagrama de alcançabilidade, a partir da Figura 2 e, também, ditado por (3.2).

Figura 3 - Padrão de alcançabilidade do exemplo 1.

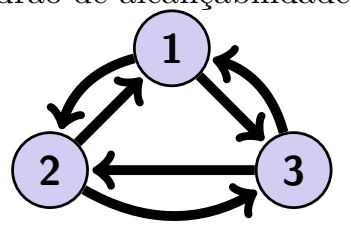

Fonte: produção do próprio autor.

Uma realização típica do processo descrito por (3.2) pode ser visto na Figura 4.

Como era de se esperar o comportamento dos descritores causais puramente linear e kernelizado são muito similares, pois, o kernel utilizado para a análise, ou seja, $\kappa(\mathbf{x}, \mathbf{y})=$ $(1+\langle\mathbf{x}, \mathbf{y}\rangle)^{2}$, contempla, além das não-lineares, a componente linear. Essa observação pode ser apreciada nas Figuras 5, 6, 7 e 8.

Nas Tabelas 1, 2 e 3 é possível observar um comparativo entre as técnicas não-kernelizadas e as técnicas kernelizadas. Utilizando-se o nível de significância de $\alpha=1 \%$, pode-se concluir, que para mapas lineares os métodos são equiparáveis e, o mais importante, vê-se que é possível capturar comportamento linear, mesmo com um kernel não-linear. 


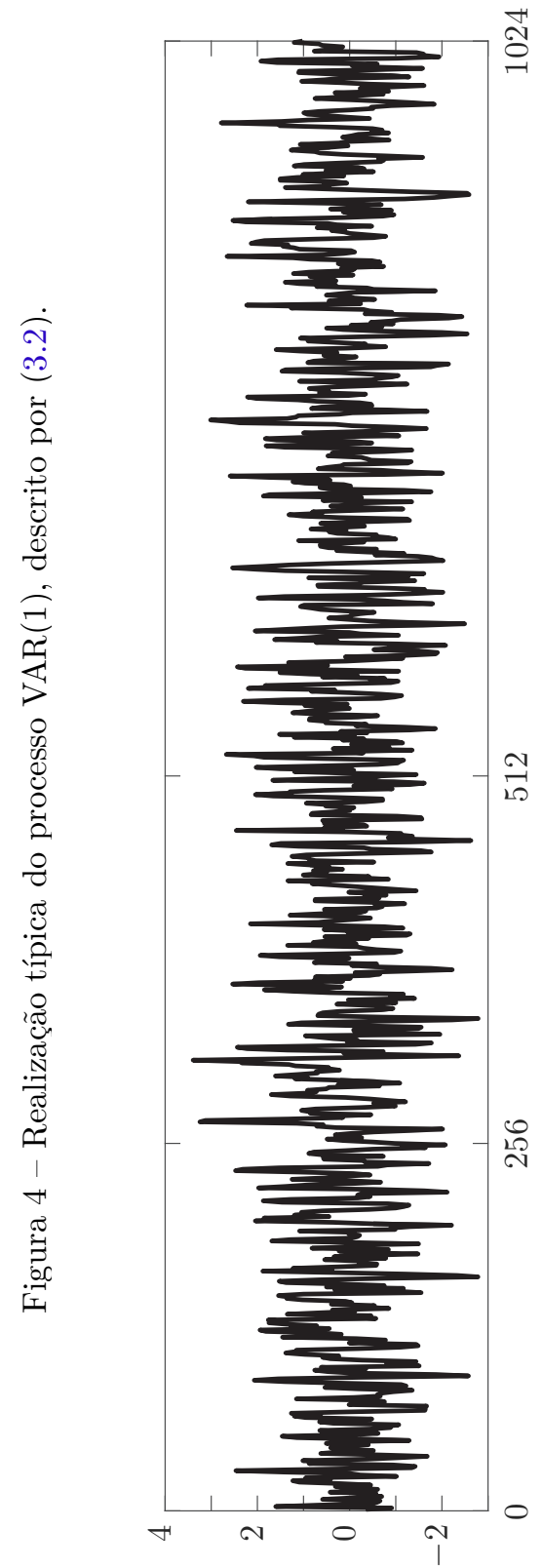

$(u)^{\operatorname{Lx} x}$

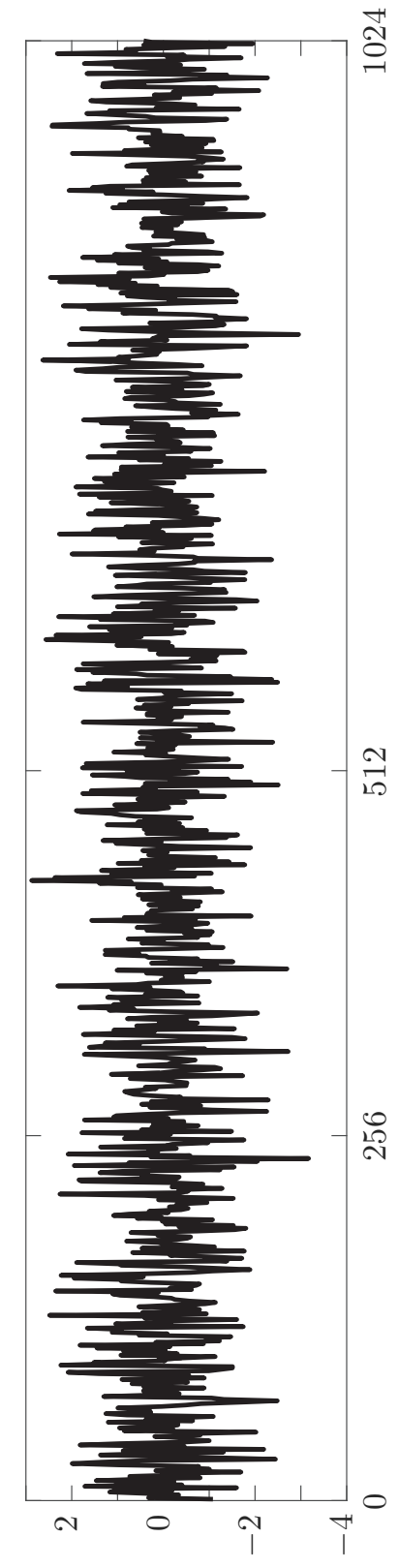

$(u)^{2} x$

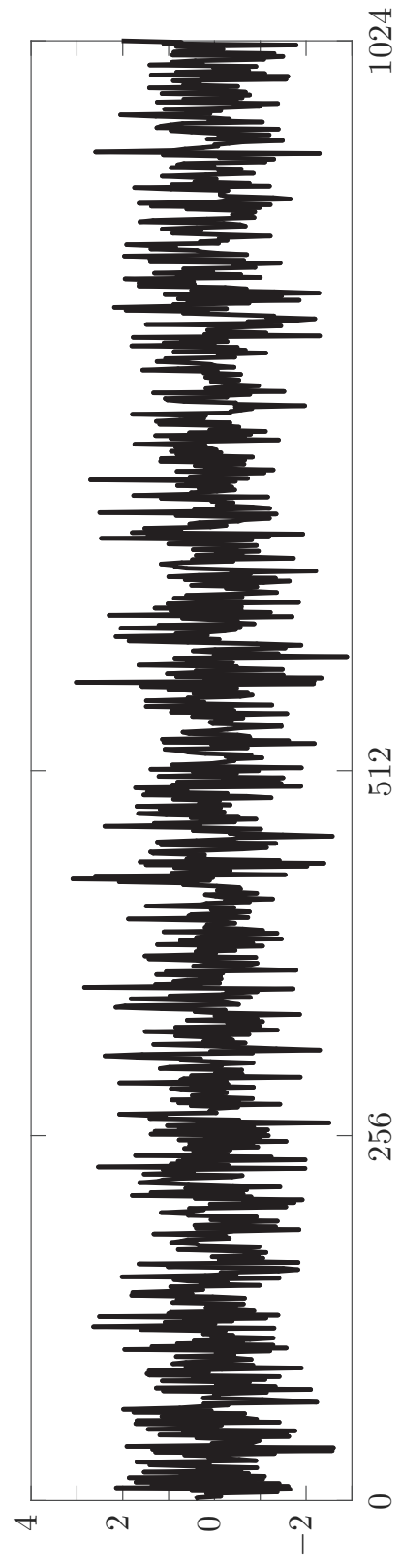

$(u)^{\varepsilon} x$ 
Figura 5 - $i$ PDC para uma realização de (3.2), considerando um nível de significância de $\alpha=0,01$. Utilizando o critério de escolha de ordem de HQ, obteve-se um modelo VAR(1).

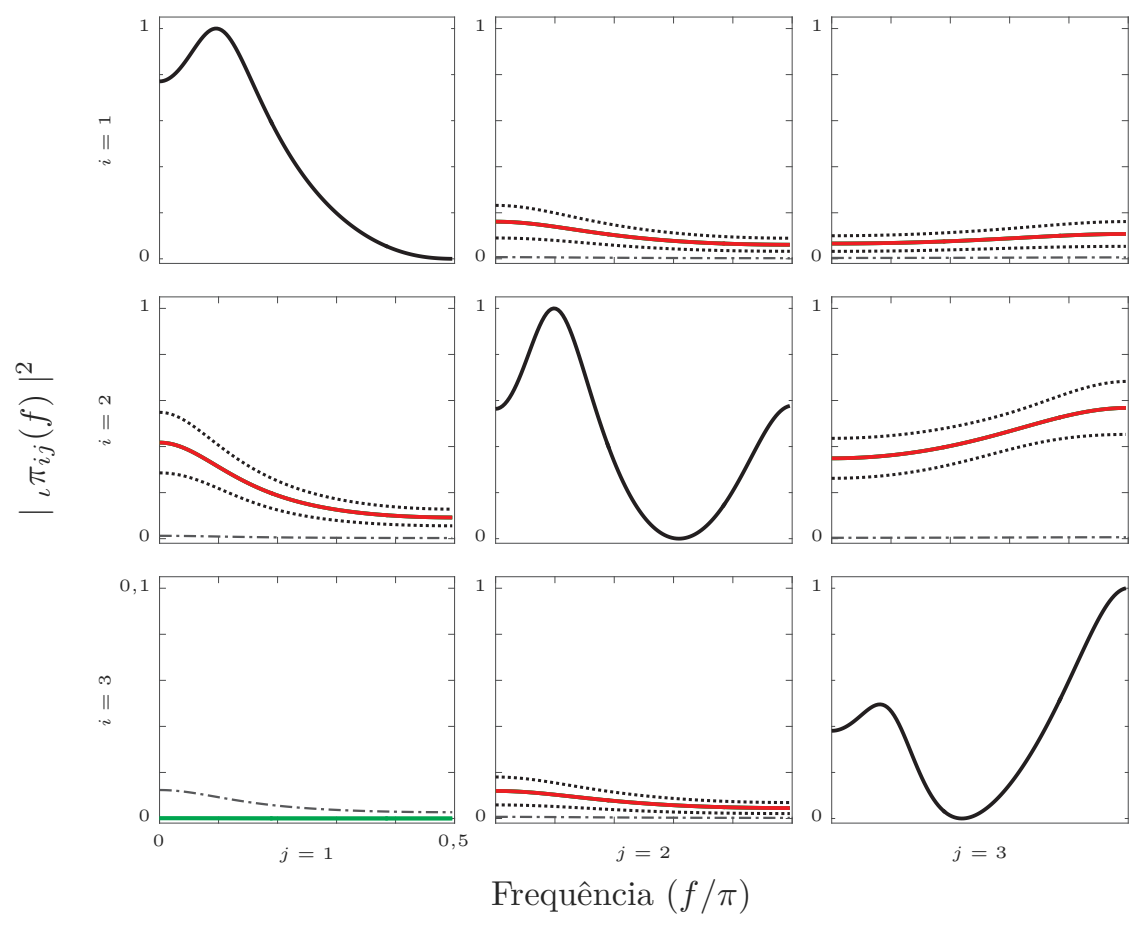

Fonte: produção do próprio autor.

Figura 6 - knPDC para uma realização de (3.2), utilizando o kernel $\kappa(\mathbf{x}, \mathbf{y})=(1+\langle\mathbf{x}, \mathbf{y}\rangle)^{2}$, considerando um nível de significância de $\alpha=0,01$. Utilizando o critério de escolha de ordem de HQ, obtevese um modelo $k \operatorname{VAR}(1)$.
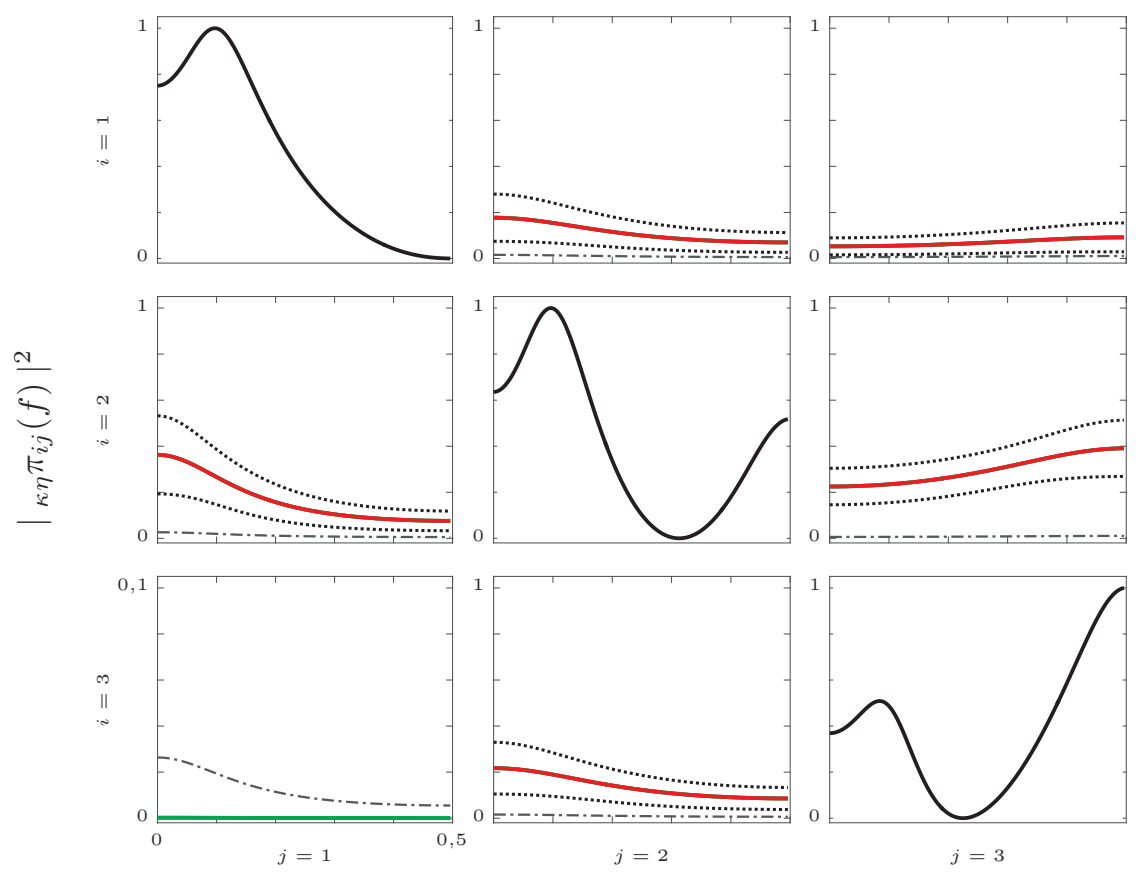

Frequência $(f / \pi)$

Fonte: produção do próprio autor. 
Figura 7 - $i$ DTF para uma realização de (3.2), considerando um nível de significância de $\alpha=0,01$. Utilizando o critério de escolha de ordem de HQ, obteve-se um modelo VAR(1).
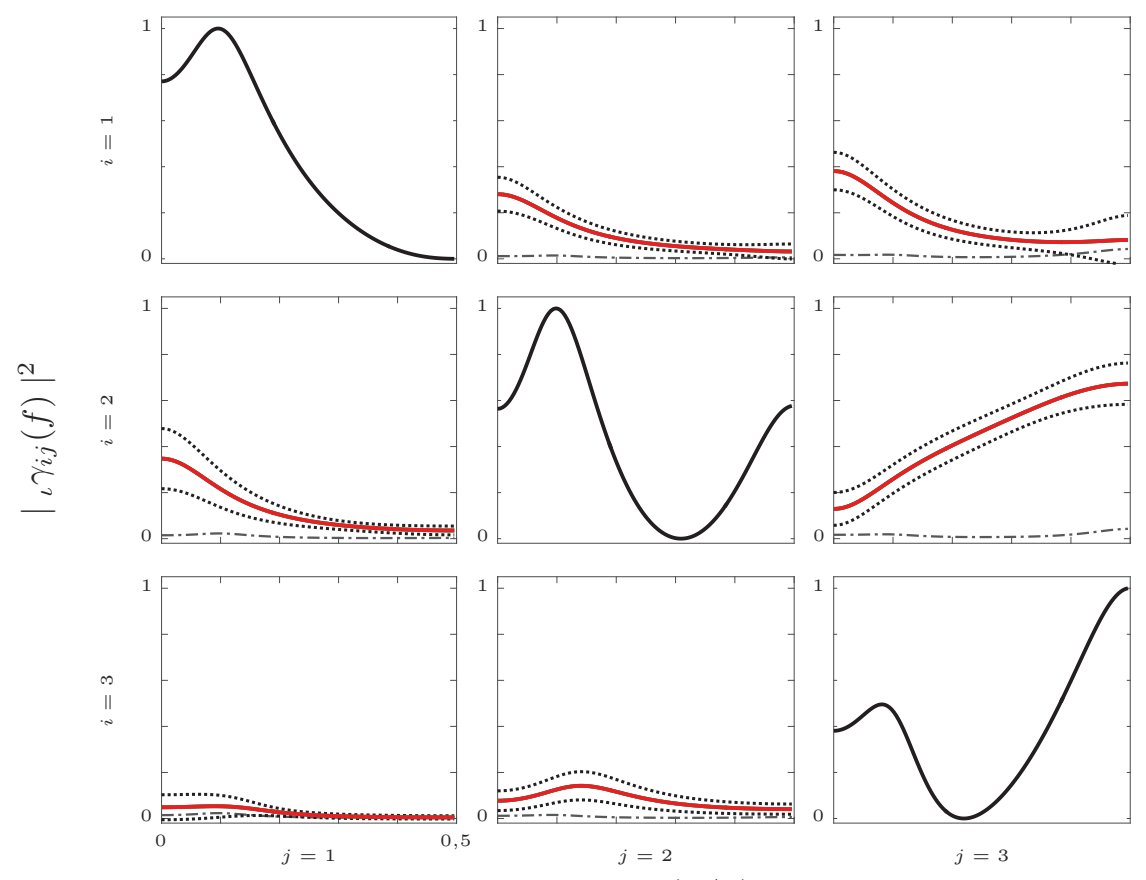

Frequência $(f / \pi)$

Fonte: produção do próprio autor.

Figura 8 - knDTF para uma realização de (3.2), utilizando o kernel $\kappa(\mathbf{x}, \mathbf{y})=(1+\langle\mathbf{x}, \mathbf{y}\rangle)^{2}$, considerando um nível de significância de $\alpha=0,01$. Utilizando o critério de escolha de ordem de HQ, obtevese um modelo $k \operatorname{VAR}(1)$.
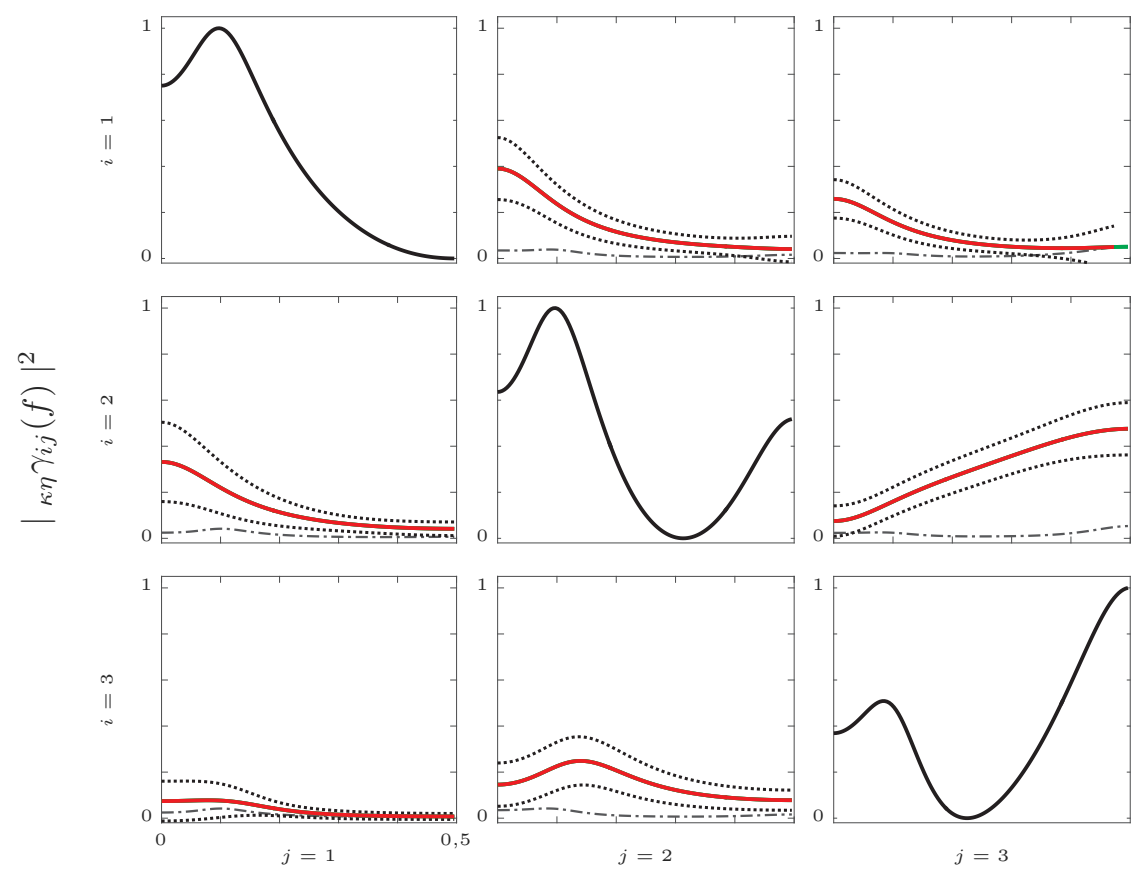

Frequência $(f / \pi)$

Fonte: produção do próprio autor. 
Tabela 1 - Comparação entre as taxas de verdadeiros positivos $(\boldsymbol{V P})$ e falsos positivos $(\boldsymbol{F} \boldsymbol{P})$, observados, de $\operatorname{GCT}\left(\mathrm{GCT}_{i j}\right)$ e $k n \mathrm{GCT}\left(k n \mathrm{GCT}_{i j}\right.$, utilizando o kernel $\left.\kappa(\mathbf{x}, \mathbf{y})=(1+\langle\mathbf{x}, \mathbf{y}\rangle)^{2}\right)$, respectivamente, para as conexões $\boldsymbol{i} \leftarrow \boldsymbol{j}$.

GCT

\begin{tabular}{|c|c|c|c|c|}
\hline & & 256 & 512 & 1024 \\
\hline \multirow{3}{*}{$V P$} & $1 \leftarrow 2$ & 100,00 & 100,00 & 100,00 \\
\hline & $2 \leftarrow 1$ & 100,00 & 100,00 & 100,00 \\
\hline & $1 \leftarrow 3$ & 100,00 & 100,00 & 100,00 \\
\hline$F P$ & $3 \leftarrow 1$ & 0,92 & 1,19 & 0,90 \\
\hline \multirow{2}{*}{$V P$} & $2 \leftarrow 3$ & 100,00 & 100,00 & 100,00 \\
\hline & $3 \leftarrow 2$ & 100,00 & 100,00 & 100,00 \\
\hline
\end{tabular}

$k n$ GCT

\begin{tabular}{|c|c|c|}
\hline 256 & 512 & 1024 \\
\hline 99,99 & 100,00 & 100,00 \\
\hline 100,00 & 100,00 & 100,00 \\
\hline 99,96 & 100,00 & 100,00 \\
\hline 0,55 & 0,53 & 0,36 \\
\hline 100,00 & 100,00 & 100,00 \\
\hline 100,00 & 100,00 & 100,00 \\
\hline
\end{tabular}

Fonte: produção do próprio autor.

Tabela 2 - Comparação entre as taxas de verdadeiros positivos $(\boldsymbol{V} \boldsymbol{P})$ e falsos positivos $(\boldsymbol{F P})$, observados, da PDC $\left(\left|\pi_{i j}(f)\right|^{2}\right)$ e $k n \operatorname{PDC}\left(\left.{ }_{\kappa \eta} \pi_{i j}(f)\right|^{2}\right.$, utilizando o kernel $\left.\kappa(\mathbf{x}, \mathbf{y})=(1+\langle\mathbf{x}, \mathbf{y}\rangle)^{2}\right)$, respectivamente, para as conexões $i \leftarrow \boldsymbol{j}$.

PDC

\begin{tabular}{|c|c|c|c|c|}
\hline & & 256 & 512 & 1024 \\
\hline \multirow{3}{*}{$V P$} & $1 \leftarrow 2$ & 100,00 & 100,00 & 100,00 \\
\hline & $2 \leftarrow 1$ & 100,00 & 100,00 & 100,00 \\
\hline & $1 \leftarrow 3$ & 100,00 & 100,00 & 100,00 \\
\hline$F P$ & $3 \leftarrow 1$ & 0,93 & 1,22 & 0,91 \\
\hline \multirow{2}{*}{$V P$} & $2 \leftarrow 3$ & 100,00 & $\overline{100,00}$ & 100,00 \\
\hline & $3 \leftarrow 2$ & $\overline{100,00}$ & 100,00 & 100,00 \\
\hline
\end{tabular}

$k n \mathrm{PDC}$

\begin{tabular}{rrr}
\multicolumn{3}{c}{$\boldsymbol{k n P D C}$} \\
\hline $\mathbf{2 5 6}$ & $\mathbf{5 1 2}$ & $\mathbf{1 0 2 4}$ \\
\hline 99,99 & 100,00 & 100,00 \\
\hline 100,00 & 100,00 & 100,00 \\
\hline 99,96 & 100,00 & 100,00 \\
\hline 0,56 & 0,57 & 0,36 \\
\hline 100,00 & 100,00 & 100,00 \\
\hline 100,00 & 100,00 & 100,00 \\
\hline
\end{tabular}

Tabela 3 - Comparação entre as taxas de verdadeiros positivos $(\boldsymbol{V} \boldsymbol{P})$ e falsos positivos $(\boldsymbol{F P})$, observados, da DTF $\left(\left|\gamma_{i j}(f)\right|^{2}\right)$ e $k n \operatorname{DTF}\left(\left.{ }_{\kappa \eta} \gamma_{i j}(f)\right|^{2}\right.$, utilizando o kernel $\kappa(\mathbf{x}, \mathbf{y})=(1+\langle\mathbf{x}, \mathbf{y}\rangle)^{2}$, respectivamente, para as conexões $\boldsymbol{i} \leftarrow \boldsymbol{j}$.

DTF

\begin{tabular}{|c|c|c|c|c|}
\hline & & 256 & 512 & 1024 \\
\hline & $1 \leftarrow 2$ & 100,00 & 100,00 & 100.00 \\
\hline & $2 \leftarrow 1$ & 100,00 & 100,00 & 100,00 \\
\hline & $1 \leftarrow 3$ & 100,00 & 100,00 & 100,00 \\
\hline$V F$ & $3 \leftarrow 1$ & 98,79 & 100,00 & 100,00 \\
\hline & $2 \leftarrow 3$ & 100,00 & $\overline{100,00}$ & 100,00 \\
\hline & $3 \leftarrow 2$ & 100,00 & 100,00 & 100,00 \\
\hline
\end{tabular}

$k n \mathrm{DTF}$

\begin{tabular}{rrr}
\hline $\mathbf{2 5 6}$ & $\mathbf{5 1 2}$ & $\mathbf{1 0 2 4}$ \\
\hline 100,00 & 100,00 & 100,00 \\
\hline 100,00 & 100,00 & 100,00 \\
\hline 100,00 & 100,00 & 100,00 \\
\hline 97,25 & 99,99 & 99,99 \\
\hline 100,00 & 100,00 & 100,00 \\
\hline 100,00 & 100,00 & 100,00 \\
\hline utor. & &
\end{tabular}


3.3.2 Exemplo 2: Modelo não-linear de banda limitada 2D

Seja o modelo não-linear, descrito pelas equações,

$$
\begin{aligned}
{\left[\begin{array}{l}
x_{1}(n) \\
x_{2}(n)
\end{array}\right]=} & {\left[\begin{array}{cc}
2 R \cos \left(2 \pi f_{0}\right) & 0 \\
0 & -R
\end{array}\right]\left[\begin{array}{l}
x_{1}(n-1) \\
x_{2}(n-1)
\end{array}\right]+\left[\begin{array}{cc}
-R^{2} & 0 \\
0 & 0
\end{array}\right]\left[\begin{array}{l}
x_{1}(n-2) \\
x_{2}(n-2)
\end{array}\right] } \\
& +\left[\begin{array}{ll}
0 & 0 \\
c & 0
\end{array}\right]\left[\begin{array}{l}
x_{1}^{2}(n-1) \\
x_{2}^{2}(n-1)
\end{array}\right]+\left[\begin{array}{l}
w_{1}(n) \\
w_{2}(n)
\end{array}\right]
\end{aligned}
$$

em que $\left\{w_{i}(n)\right\}_{n \in \mathbb{Z}}$ são processos de inovações i.i.d. normais, padrões.

Observe que a equação de $\left\{x_{1}(n)\right\}_{n \in \mathbb{Z}}$ descreve um oscilador de alto índice de mérito $\left(R=0,99, f_{0}=0,1\right)$, ou seja, possui uma frequência de ressonância bem definida e está acoplada de maneira quadrática a $\left\{x_{2}(n)\right\}_{n \in \mathbb{Z}}$, que é um filtro passa-baixas, no qual a conectividade pode ser aferida através do parâmetro $c$.

Um grafo, que resume o sentido de fluxo de informação entre as séries, imposto por (3.3) pode ser apreciado na Figura 9.

Figura 9 - Padrão de conectividade e alcançabilidade do exemplo 2.

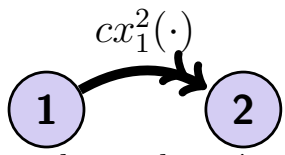

Fonte: produção do próprio autor.

No que diz respeito às simulações, permitiu-se que o fator de acoplamento $c$ variasse no conjunto $\{0,1 ; 0,5 ; 1,0\}$. Na Figura 10 é possível observar uma realização do processo de banda estreita dado por (3.3), simulado com fator de acoplamento $c=1,0$ e um número de amostras de $N=1024$. Observe que a PDC e a DTF, apesar de iguais (devido a ser um caso bivariado), são muito difíceis de serem capturas corretamente, segundo a Figura 9. Isso se deve ao acoplamento entre as séries ser não-linear e polinomial de ordem (especialmente) par [14]. Esse fato pode ser observado nas Figuras 11 e 12.

Assim, no intuito de se determinar a correta direção do fluxo de informação, faz-se o mapeamento indireto, como discutido no Capítulo 2 e se calcula as $k n \mathrm{GCT}, k n \mathrm{PDC}$, $k n$ DTF. Os dois últimos podem ser apreciados nas Figuras 13 e 14, sempre utilizando o kernel monomial quadrático, ou seja,

$$
\kappa(\mathbf{x}, \mathbf{y})=\langle\mathbf{x}, \mathbf{y}\rangle^{2}
$$

Agora, escolhendo um $\alpha=1 \%$, mostram-se as Tabelas 4, 5 e 6, resumindo o comportamento quanto à detecção estatística. Nestas, indicam-se as porcentagens de verdadeiro positivo (VP) e falso positivo (FP), mostrando as respectivas taxas em que ocorrem nas replicações de Monte Carlo, fazendo-se uma comparação entre os kerneis $\kappa(\mathbf{x}, \mathbf{y})=\langle\mathbf{x}, \mathbf{y}\rangle^{2}$ e $\kappa(\mathbf{x}, \mathbf{y})=\langle\mathbf{x}, \mathbf{y}\rangle^{4}$. Uma comparação análoga pode ser apreciada em [55], feita pelo próprio autor. 
Assim, observa-se que (3.3) é reconstruído corretamente, na maior parte do tempo, utilizando-se (preferencialmente) (3.4), para os métodos kernelizados. Antes, isso era quase impossível de se fazer com a GCT, PDC e DTF ordinárias. 


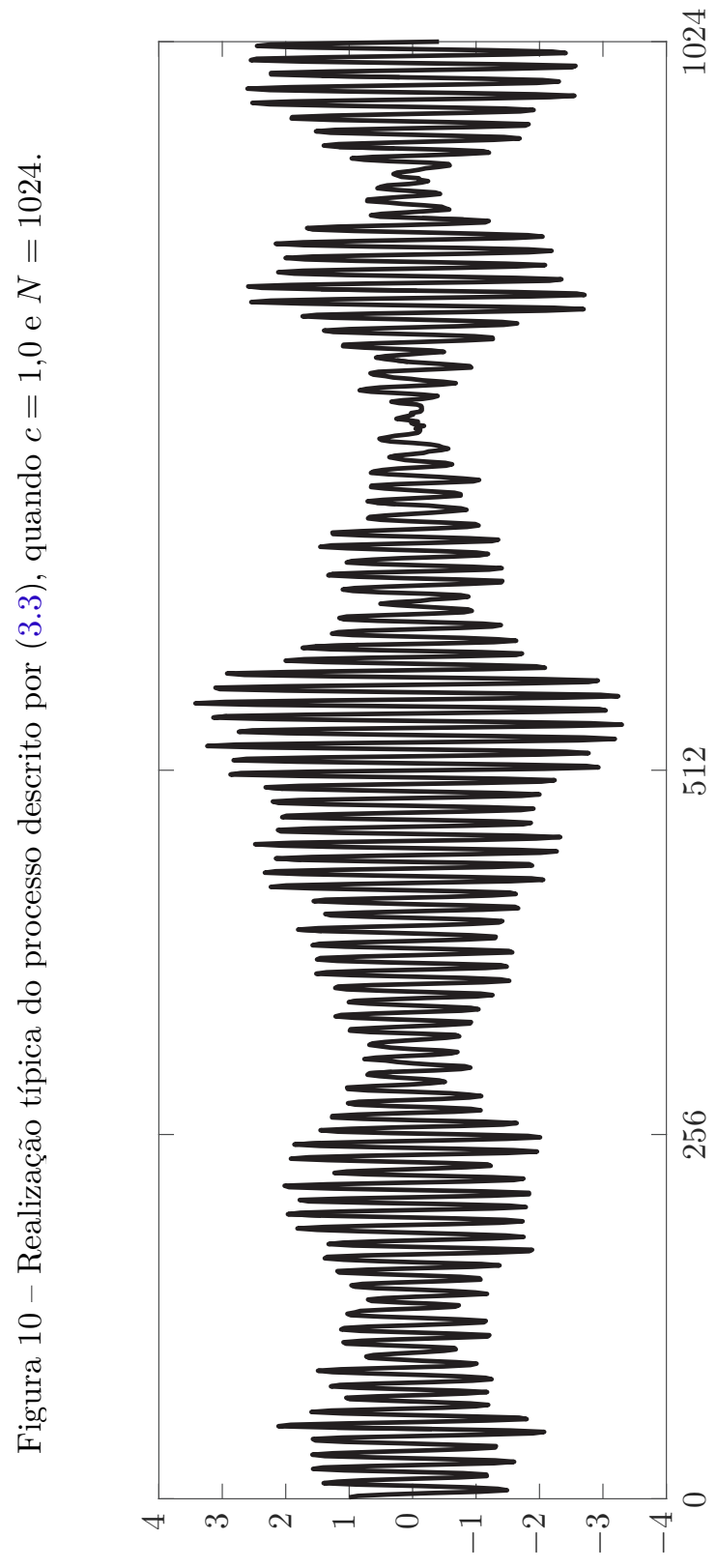

$(u)^{\mathrm{\perp} x}$

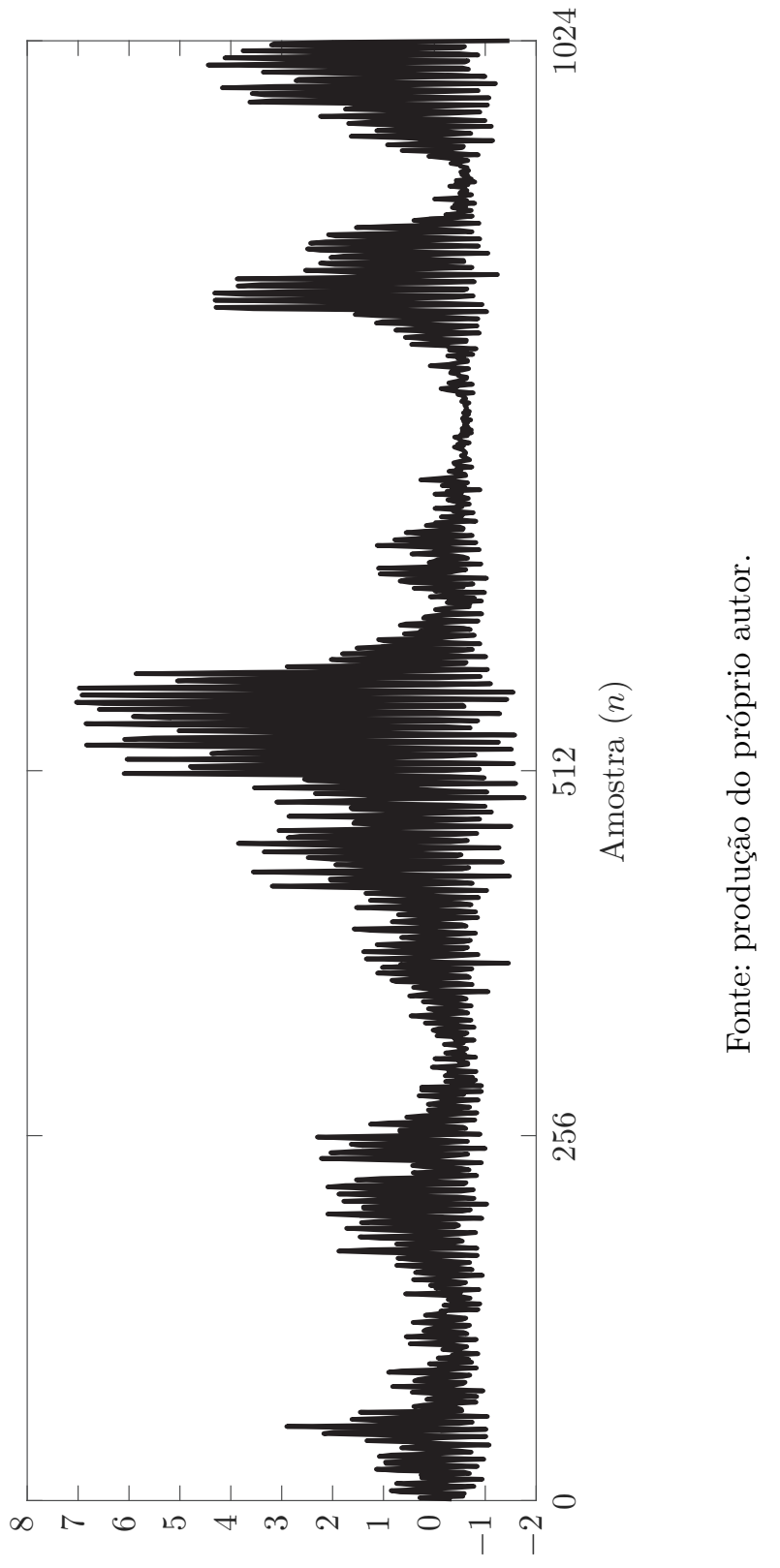

$(u)^{2} x$ 
Figura 11 - knPDC para uma realização de (3.3), considerando um nível de significância de $\alpha=0,01$. Para a escolha da ordem do modelo, utilizou-se o critério HQ, que gerou um VAR(2).

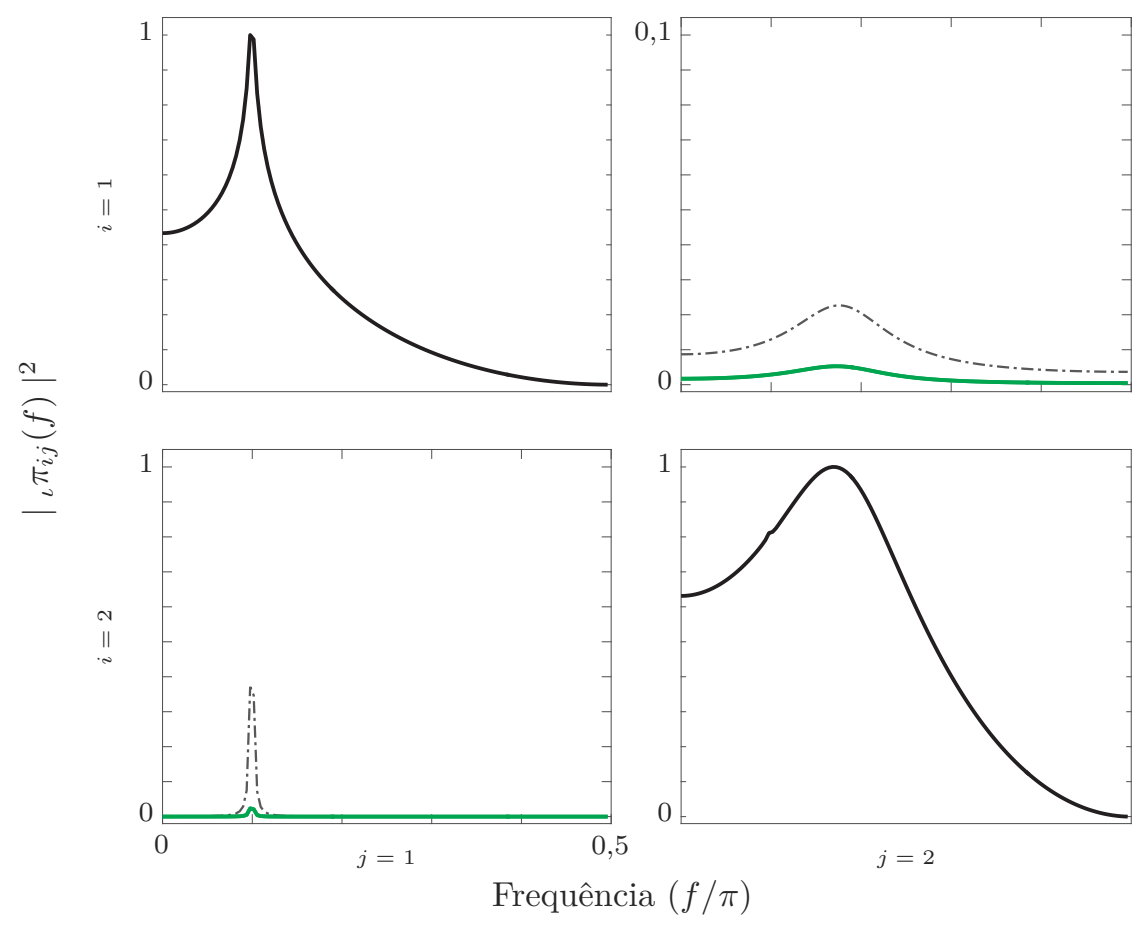

Fonte: produção do próprio autor.

Figura 12 - knDTF para uma realização de (3.3), considerando um nível de significância de $\alpha=0,01$. Para a escolha da ordem do modelo, utilizou-se o critério HQ, que gerou um $\operatorname{VAR}(2)$.

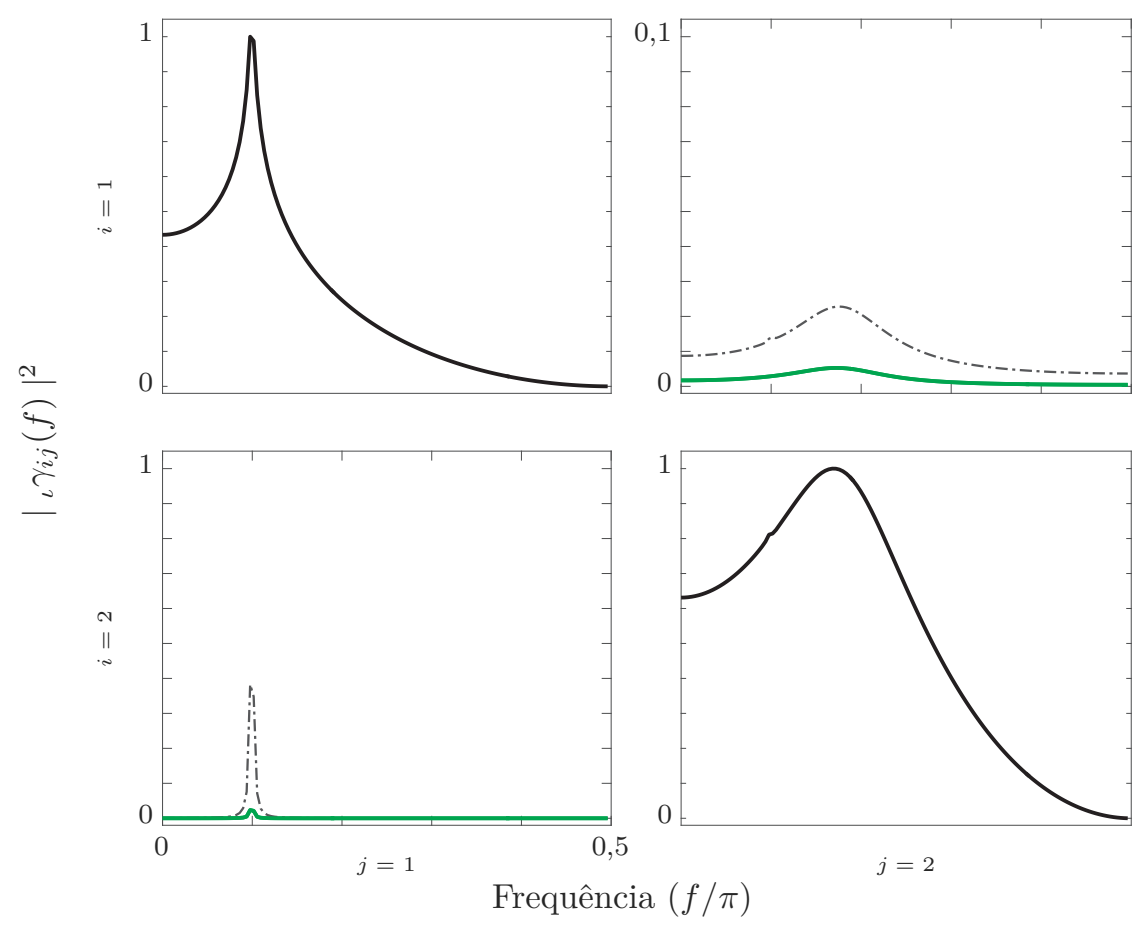

Fonte: produção do próprio autor. 
Figura 13 - knPDC para uma realização de (3.3), utilizando o kernel $\kappa(\mathbf{x}, \mathbf{y})=\langle\mathbf{x}, \mathbf{y}\rangle^{2}$, considerando um nível de significância de $\alpha=0,01$. Para a escolha da ordem do modelo, utilizou-se o critério $\mathrm{HQ}$, que gerou um $k \operatorname{VAR}(9)$.

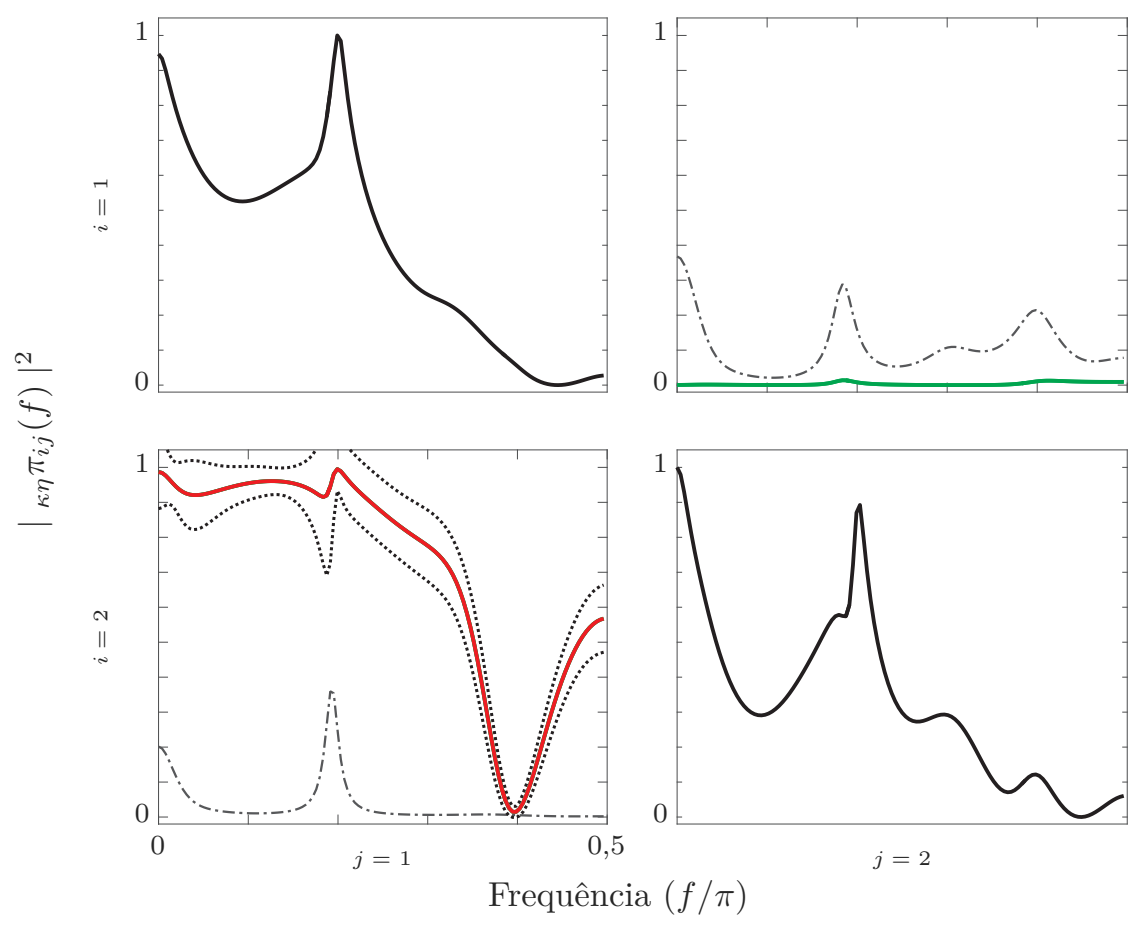

Fonte: produção do próprio autor.

Figura $14-k n$ DTF para uma realização de (3.3), utilizando o kernel $\kappa(\mathbf{x}, \mathbf{y})=\langle\mathbf{x}, \mathbf{y}\rangle^{2}$, considerando um nível de significância de $\alpha=0,01$. Para a escolha da ordem do modelo, utilizou-se o critério $\mathrm{HQ}$, que gerou um $k \mathrm{VAR}(9)$.

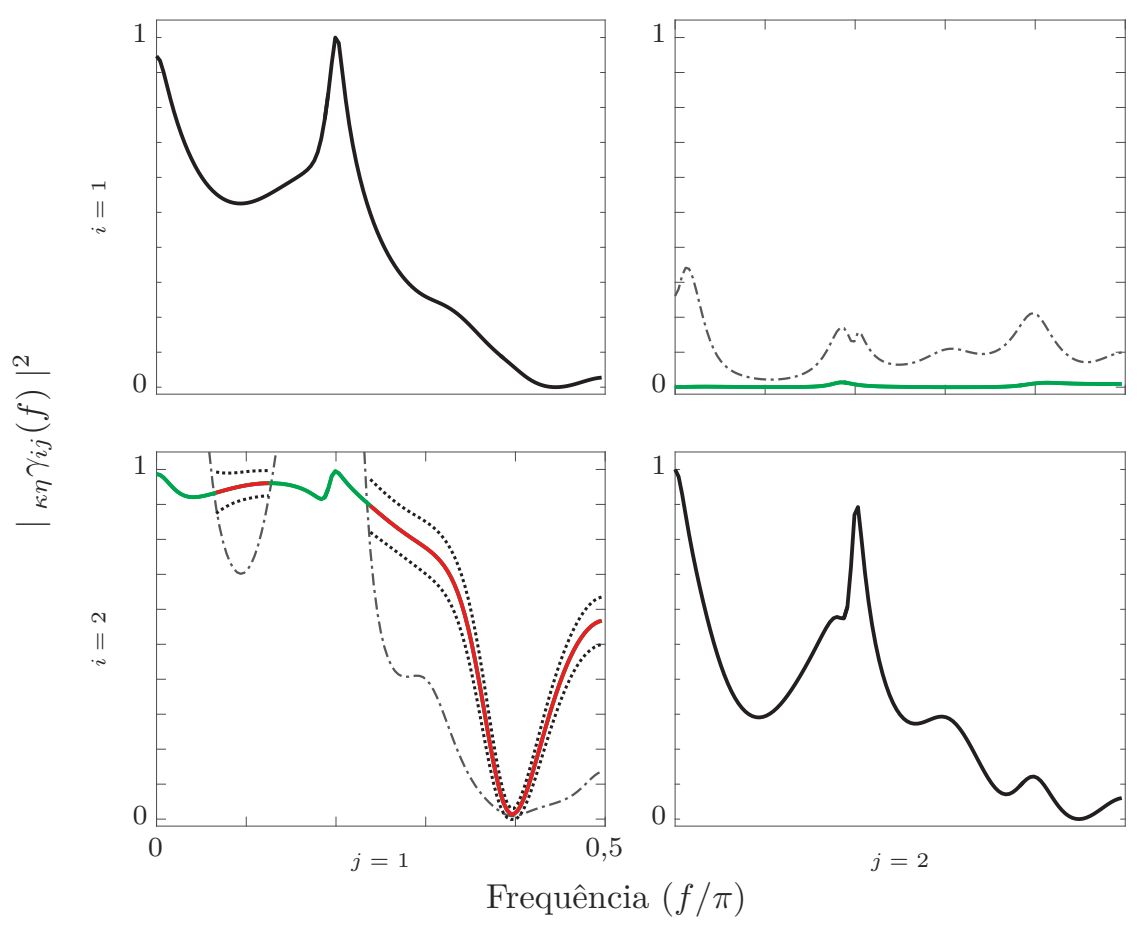

Fonte: produção do próprio autor. 
Tabela 4 - Tabela de contingência para o exemplo 2, referente ao $k n$ GCT.

\begin{tabular}{|c|c|c|c|c|c|c|c|}
\hline & & \multicolumn{3}{|c|}{$V P-100 \times(1-\widehat{\beta})$} & \multicolumn{3}{|c|}{$F P-100 \times(\widehat{\alpha})$} \\
\hline Kernel & & 256 & 512 & 1024 & 256 & 512 & 1024 \\
\hline \multirow{3}{*}{$\kappa(\mathbf{x}, \mathbf{y})=\langle\mathbf{x}, \mathbf{y}\rangle^{2}$} & 0,10 & 99,98 & 100,00 & 100,00 & 0,06 & 0,01 & 0,00 \\
\hline & 0,50 & 100,00 & 100,00 & 100,00 & 0,14 & 0,01 & 0,00 \\
\hline & 1,00 & 100,00 & 100,00 & 100,00 & 0,15 & 0,01 & 0,00 \\
\hline \multirow{3}{*}{$\kappa(\mathbf{x}, \mathbf{y})=\langle\mathbf{x}, \mathbf{y}\rangle^{4}$} & 0,10 & 99,97 & 100,00 & 100,00 & 2,12 & 0,04 & 0,08 \\
\hline & 0,50 & 100,00 & 100,00 & 100,00 & 1,27 & 0,31 & 0,04 \\
\hline & 1,00 & 100,00 & 100,00 & 100,00 & 1,24 & 0,33 & 0,04 \\
\hline
\end{tabular}

Fonte: produção do próprio autor.

Tabela 5 - Tabela de contingência para o exemplo 2, referente ao $k n$ PDC.

\begin{tabular}{|c|c|c|c|c|c|c|c|}
\hline & & \multicolumn{3}{|c|}{$V P-100 \times(1-\widehat{\beta})$} & \multicolumn{3}{|c|}{$F P-100 \times(\widehat{\alpha})$} \\
\hline Kernel & & 256 & 512 & 1024 & 256 & 512 & 1024 \\
\hline \multirow{3}{*}{$\kappa(\mathbf{x}, \mathbf{y})=\langle\mathbf{x}, \mathbf{y}\rangle^{2}$} & 0,10 & 99,94 & 100,00 & 100,00 & 0,08 & 0,02 & 0,00 \\
\hline & 0,50 & 100,00 & 100,00 & 100,00 & 0,19 & 0,02 & 0,00 \\
\hline & 1,00 & 100,00 & 100,00 & 100,00 & 0,18 & 0,02 & 0,00 \\
\hline \multirow{3}{*}{$\kappa(\mathbf{x}, \mathbf{y})=\langle\mathbf{x}, \mathbf{y}\rangle^{4}$} & 0,10 & 99,97 & 100,00 & 100,00 & 3,35 & 0,82 & 0,12 \\
\hline & 0,50 & 100,00 & 100,00 & 100,00 & 1,84 & 0,42 & 0,05 \\
\hline & 1,00 & 100,00 & 100,00 & 100,00 & 1,84 & 0,46 & 0,04 \\
\hline
\end{tabular}

Fonte: produção do próprio autor.

Tabela 6 - Tabela de contingência para o exemplo 2, referente ao $k n$ DTF.

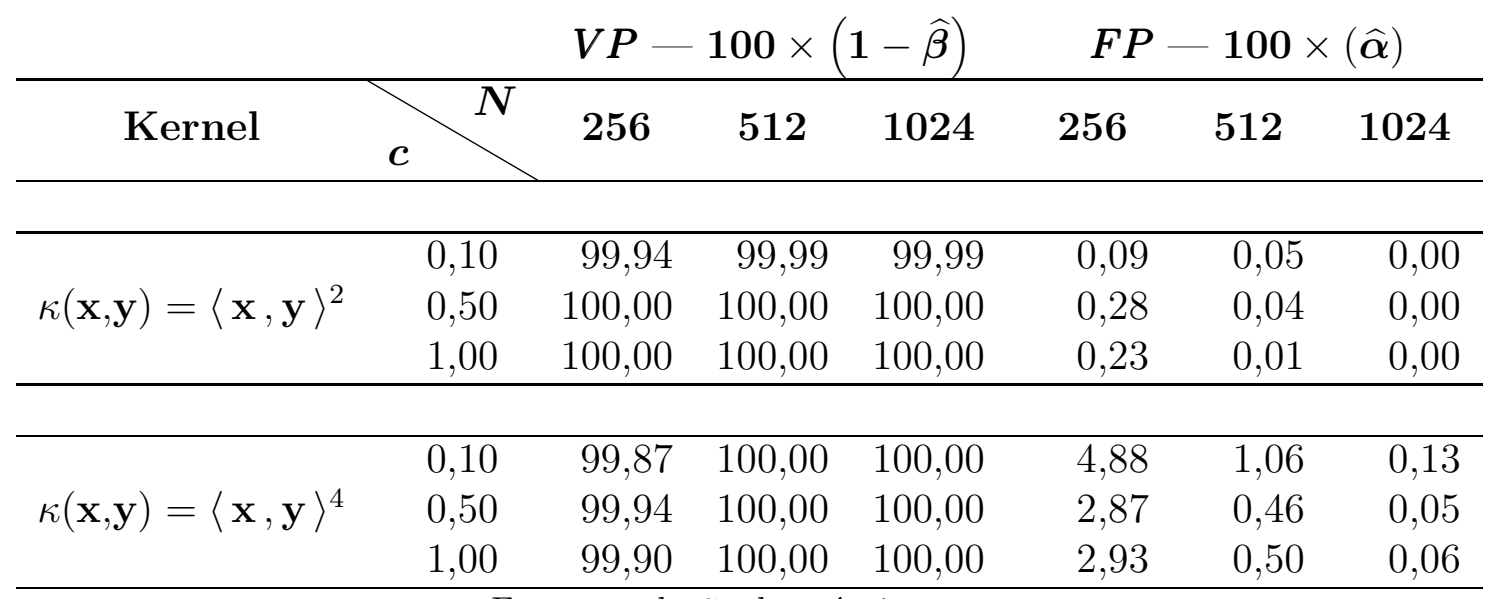




\subsubsection{Exemplo 3: Modelo não-linear de banda larga 2D}

Seja o mapa, retirado de $[17,65]$,

$$
\left\{\begin{array}{l}
x_{1}(n)=3,4 x_{1}(n-1)\left[1-x_{1}^{2}(n-1)\right] \mathrm{e}^{-x_{1}^{2}(n-1)}+0,8 x_{1}(n-2) \\
x_{2}(n)=3,4 x_{2}(n-1)\left[1-x_{2}^{2}(n-1)\right] \mathrm{e}^{-x_{2}^{2}(n-1)}+0,5 x_{2}(n-2)+c x_{1}^{2}(n-2)
\end{array}\right.
$$

que produz sinais caóticos de banda larga, com bacia de atração no intervalo [0,1]. As simulações foram feitas considerando que o fator de acoplamento, $c$, variando no conjunto $\{0,50 ; 0,75 ; 1,00\}$.

Um exame mais apurado das realizações de (3.5), por meio do teste de memória longa Rescaled Range (R/S) de Lo [68], mostra que as séries exibem um comportamento com dependencia de longa duração e, portanto, é necessário que se realize diferenciação fracionária dos sinais, para que atinjam estacionariedade, de maneira satisfatória, atendendo a condição (2.4).

Para tanto, faz-se necessário o cálculo do expoente de Hurst das séries, que está diretamente relacionado com o parâmetro de diferenciação fracionária (ou de memória longa). Aqui, para tais estimativas, usam-se métodos baseados em wavelets, que foram implementados pelo próprio autor $[69,70]$, baseado em trabalhos de Percival, Walden e Whitcher $[71,72]$.

Uma amostra representativa de (3.5) é traçada na Figura 16. Repare que o sinal $x_{1}(n)$ possui duas regiões de operação distintas, alternando entre elas, assemelhando-se ao atrator de Lorenz [17]. Os atratores de ambos sinais, reconstruídos, são ilustrados, respectivamente, nas Figuras 17a e 17b. Ainda, na Figura 17c, mostra-se o gráfico de dispersão entre os sinais, para ilustrar que há uma correlação entre os sinais, embora pareça ser fraca.

As Figuras 18 e 19 retratam, respectivamente, a $i$ PDC e a $i$ DTF, baseadas no modelamento linear da realização, no espaço de entrada ( $c=1,0$ e $N=1024)$, após a diferenciação fracionária, sob o critério assintótico (com $\alpha=1 \%$ ) de [23], mostrando a inabilidade de se capturar a correta direção do acoplamento dado pelo seguinte diagrama de conectividade e alcançabilidade da Figura 15.

Figura 15 - Padrão de conectividade e alcançabilidade do exemplo 3.

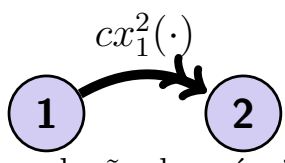

Fonte: produção do próprio autor.

As Figuras 20 e 21 mostram, respectivamente, a $k n$ PDC e $k n$ DTF utilizando o kernel $\kappa(\mathbf{x}, \mathbf{y})=\langle\mathbf{x}, \mathbf{y}\rangle^{4}$, para os mesmo dados da Figura 16. É imediato observar que a detecção correta da conectividade é obtida como para ambos $k n$ PDC e $k n$ DTF, pois, no 
gráfico, o traçado vermelho, indicando $\phi\left[x_{1}(n)\right] \rightarrow \phi\left[x_{2}(n)\right]$, está sobre o limiar, em preto e tracejado. Note que a ausência do fluxo de informação é confirmada pelo traçado verde, abaixo do limiar.

Nas Tabelas 7, 8 e 9 mostram-se detalhes do procedimento de simulação, em função do fator de acoplamento $c$ e do número de $\operatorname{amostras} N$, para as 10.000 replicações de Monte Carlo. 


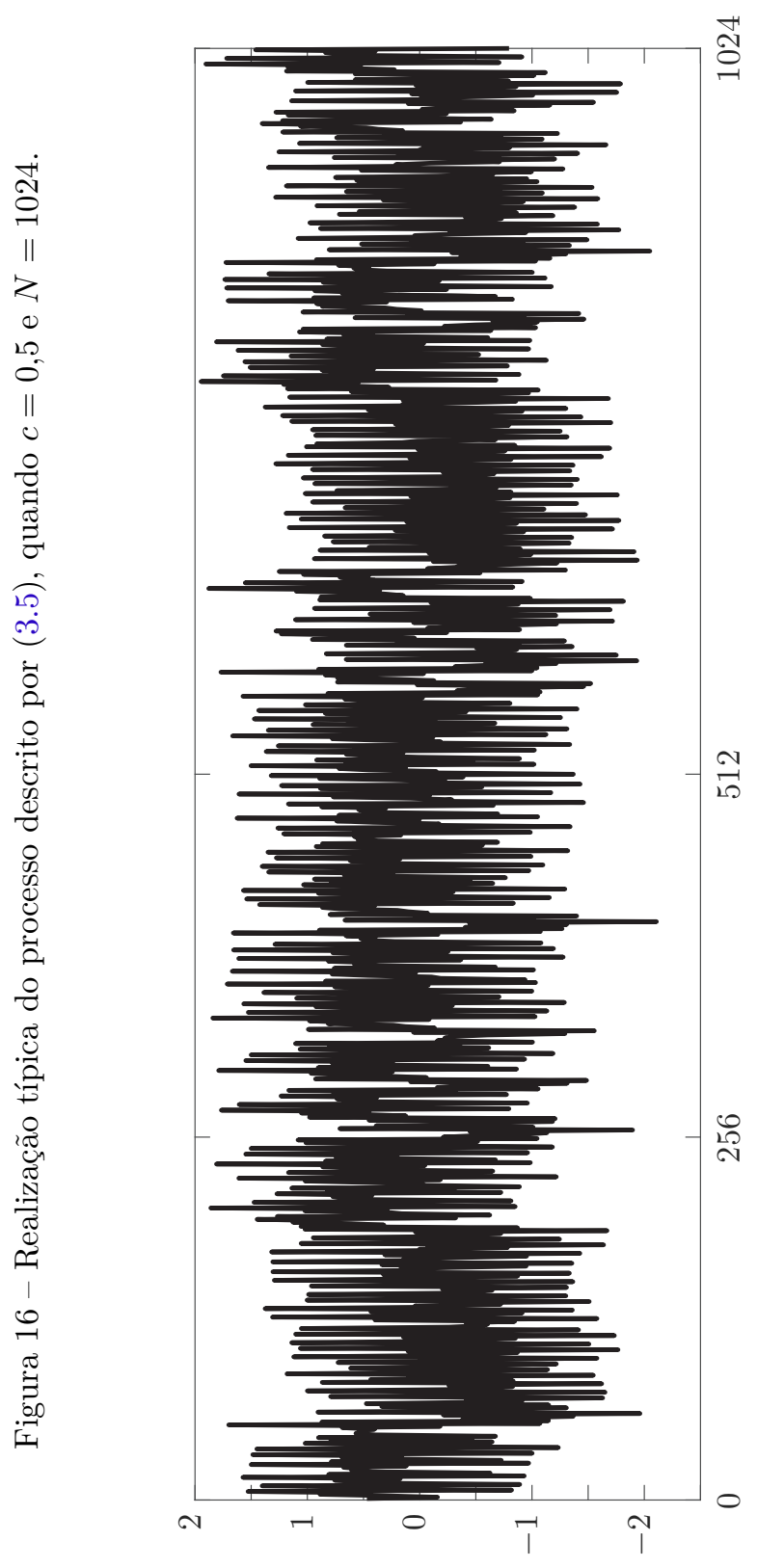

$(u)^{\complement} \mathrm{x}$

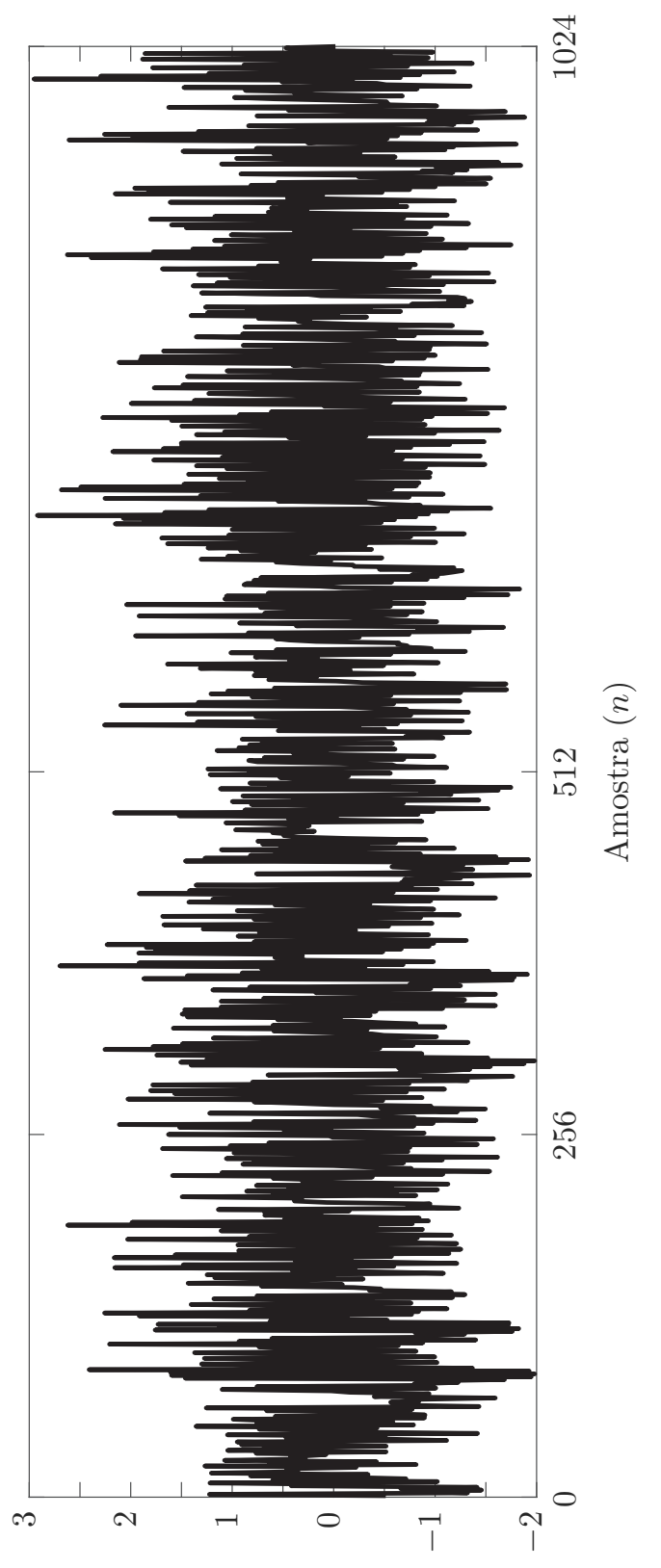

$(u)^{3} x$ 
Figura 17 - Reconstrução dos atratores de $x(n)$ e $y(n)$, nas Figuras 17a e 17b, respectivamente e um gráfico de dispersão de $x(n)$ vs. $y(n)$, na Figura 17 c.

(a) Mapa de primeiro retorno de $x(n)$.

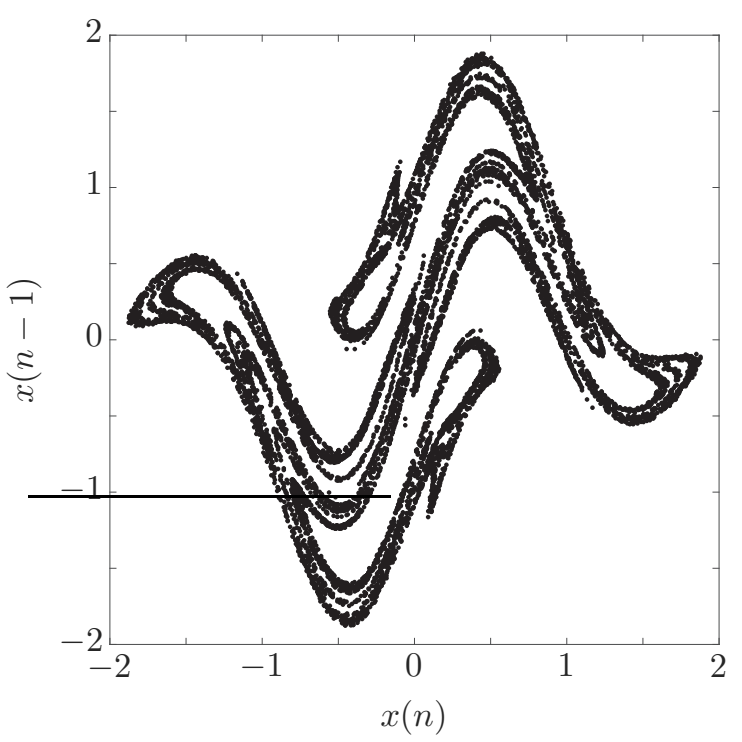

(b) Mapa de primeiro retorno de $y(n)$.

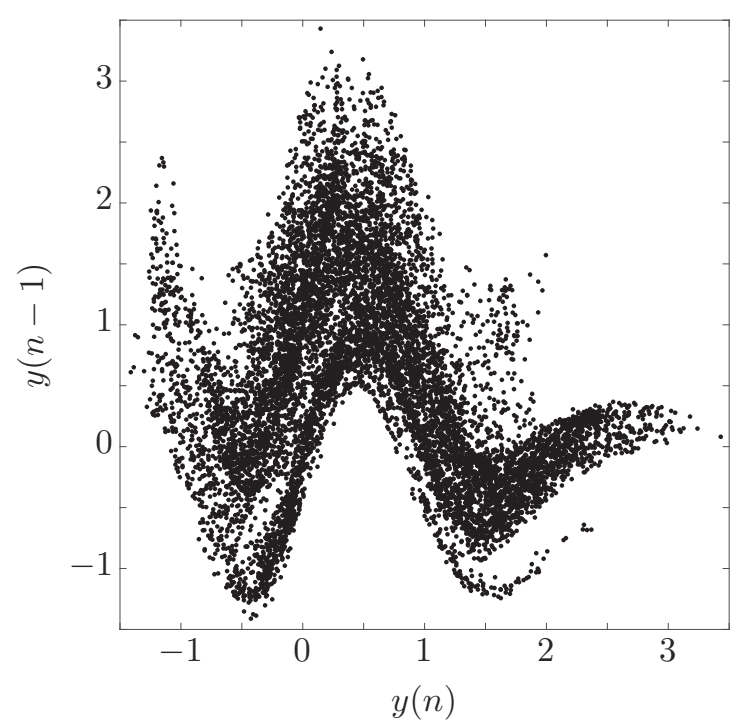

(c) Gráfico de dispersão de $x(n)$ vs. $y(n)$.

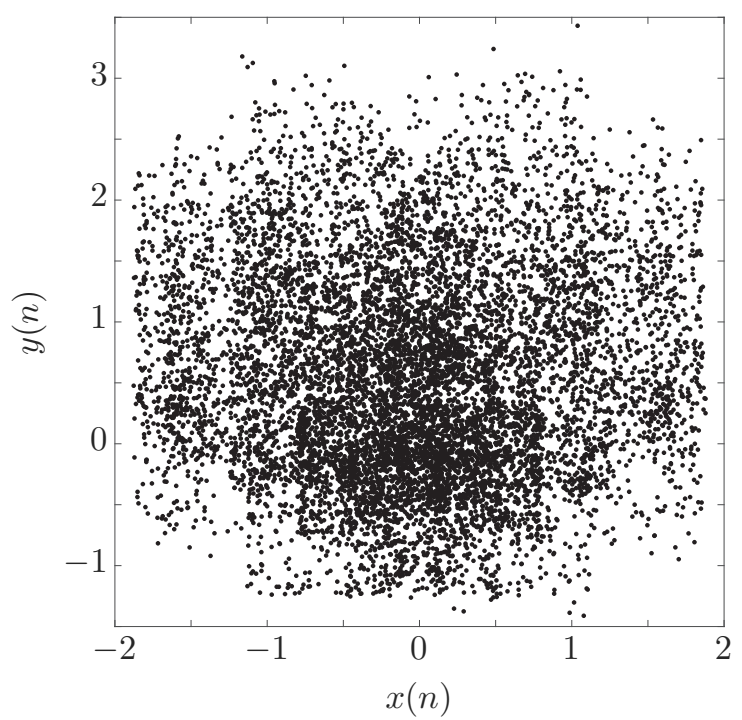

Fonte: produção do próprio autor. 
Figura 18 - $i$ PDC para uma realização de (3.5), considerando um nível de significância de $\alpha=0,01$. Para a escolha da ordem do modelo, utilizou-se o critério HQ, que gerou um $\operatorname{VAR}(2)$.

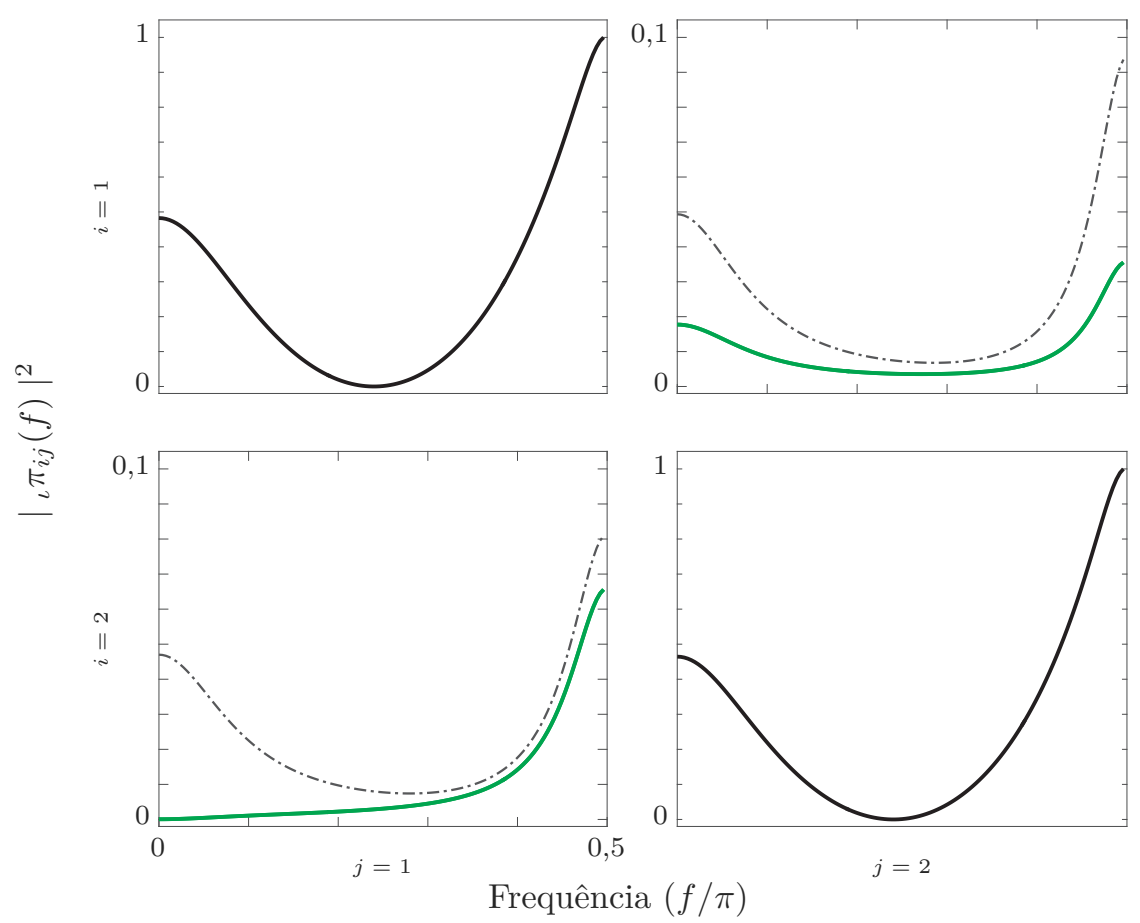

Fonte: produção do próprio autor.

Figura 19 - $i$ DTF para uma realização de (3.5), considerando um nível de significância de $\alpha=0,01$. Para a escolha da ordem do modelo, utilizou-se o critério HQ, que gerou um $\operatorname{VAR}(2)$.

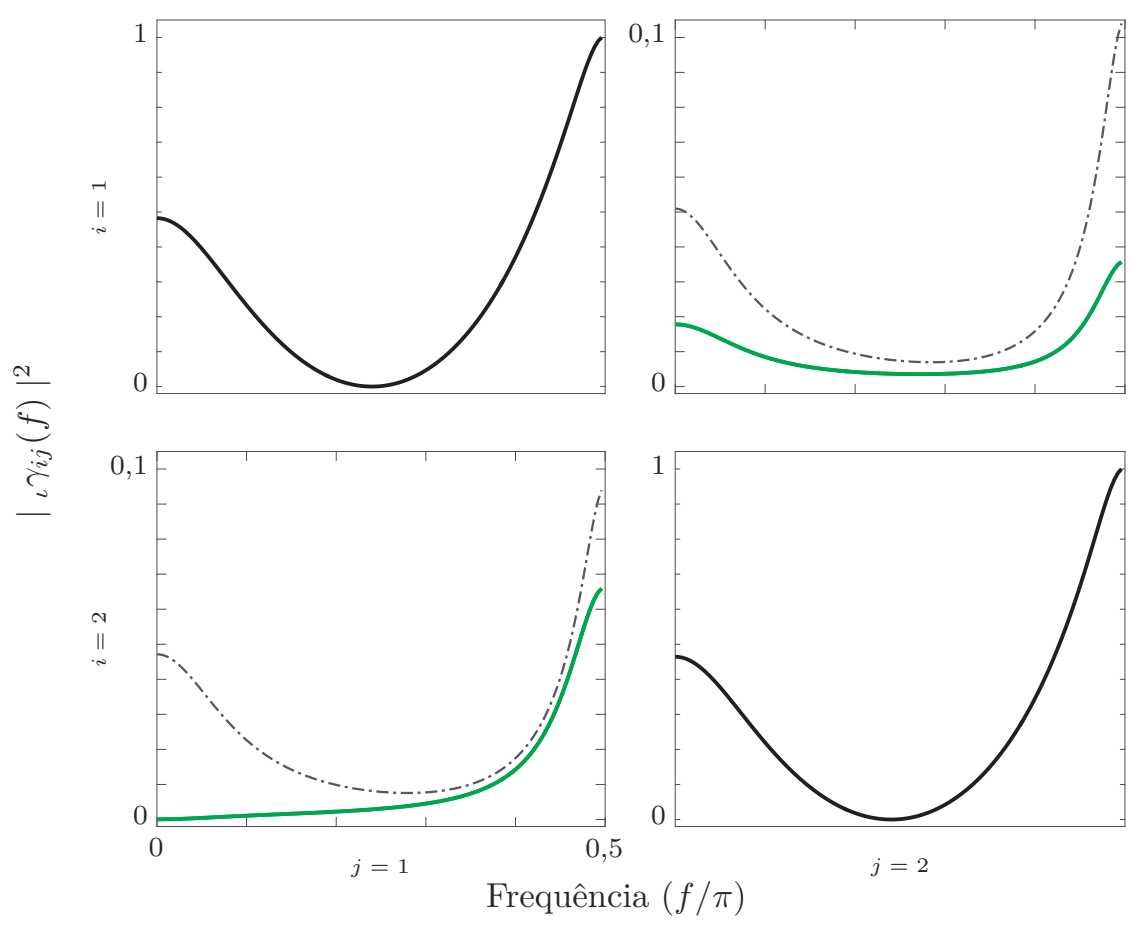

Fonte: produção do próprio autor. 
Figura 20 - knPDC para uma realização de (3.5), utilizando o kernel $\kappa(\mathbf{x}, \mathbf{y})=\langle\mathbf{x}, \mathbf{y}\rangle^{4}$, considerando um nível de significância de $\alpha=0,01$. Para a escolha da ordem do modelo, utilizou-se o critério HQ, que gerou um $k \operatorname{VAR}(2)$.
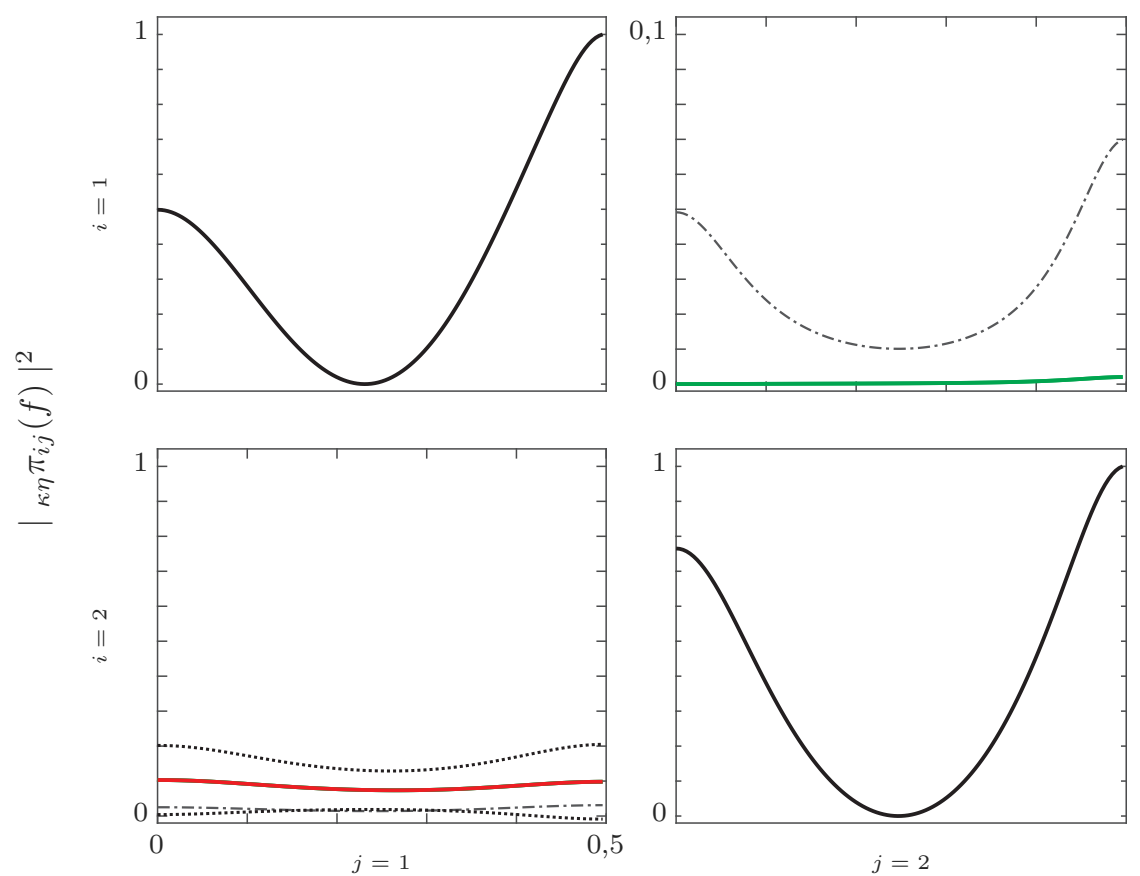

Frequência $(f / \pi)$

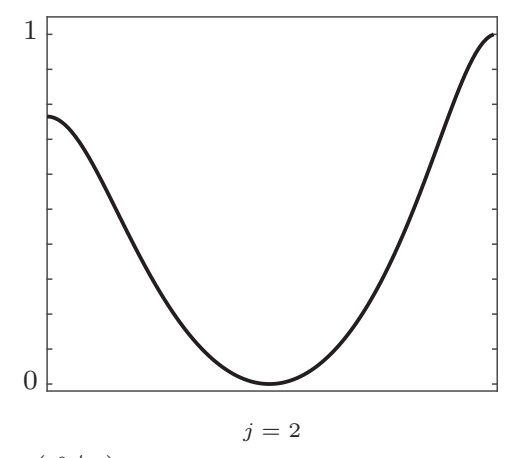

Fonte: produção do próprio autor.

Figura 21 - knDTF para uma realização de (3.5), utilizando o kernel $\kappa(\mathbf{x}, \mathbf{y})=\langle\mathbf{x}, \mathbf{y}\rangle^{4}$, considerando um nível de significância de $\alpha=0,01$. Para a escolha da ordem do modelo, utilizou-se o critério $\mathrm{HQ}$, que gerou um $k \operatorname{VAR}(2)$.
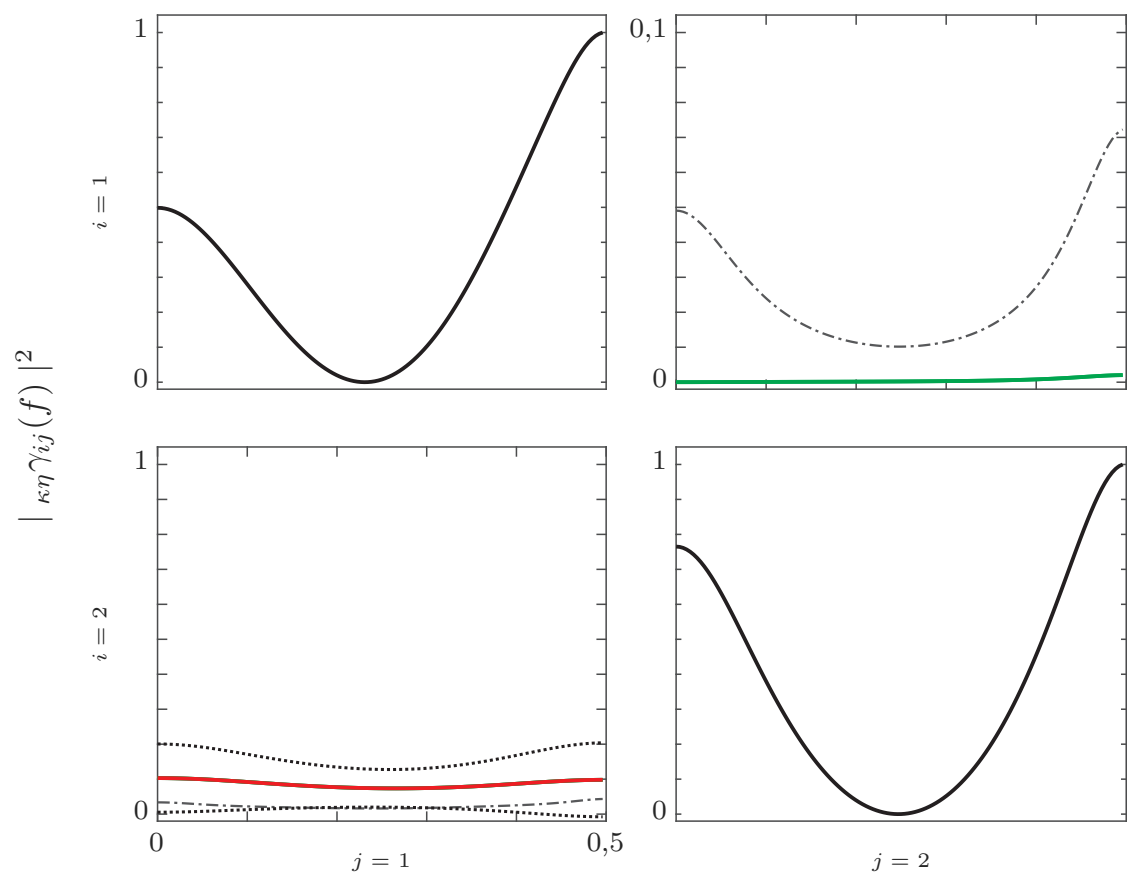

Frequência $(f / \pi)$

Fonte: produção do próprio autor. 
Tabela 7 - Tabela de contingência para o exemplo 3, referente ao $k n$ GCT.

\begin{tabular}{|c|c|c|c|c|c|c|c|}
\hline & & $V P$ & $100 \times$ & $-\widehat{\boldsymbol{\beta}})$ & $F P$ & 100 & $(\widehat{\boldsymbol{\alpha}})$ \\
\hline Kernel & & 256 & 512 & 1024 & 256 & 512 & 1024 \\
\hline & 0,50 & 95,49 & 94,40 & 100,00 & 4,11 & 5,48 & 5,67 \\
\hline$\kappa(\mathbf{x}, \mathbf{y})=\langle\mathbf{x}, \mathbf{y}\rangle^{4}$ & 0,75 & 94,65 & 100,00 & 98,12 & 0,79 & 3,21 & 4,86 \\
\hline & 1,00 & 94,87 & 95,43 & 98,17 & 0,63 & 1,53 & 1,00 \\
\hline & 0,50 & 93,78 & 95,01 & 99,99 & 3,89 & 8,41 & 4,63 \\
\hline$\kappa(\mathbf{x}, \mathbf{y})=\langle\mathbf{x}, \mathbf{y}\rangle^{8}$ & 0,75 & 95,02 & 98,25 & 96,76 & 0,73 & 0,61 & 0,42 \\
\hline & 1,00 & 90,02 & 96,60 & 96,98 & 0,02 & 2,95 & 1,04 \\
\hline
\end{tabular}

Fonte: produção do próprio autor.

Tabela 8 - Tabela de contingência para o exemplo 3 , referente ao $k n$ PDC.

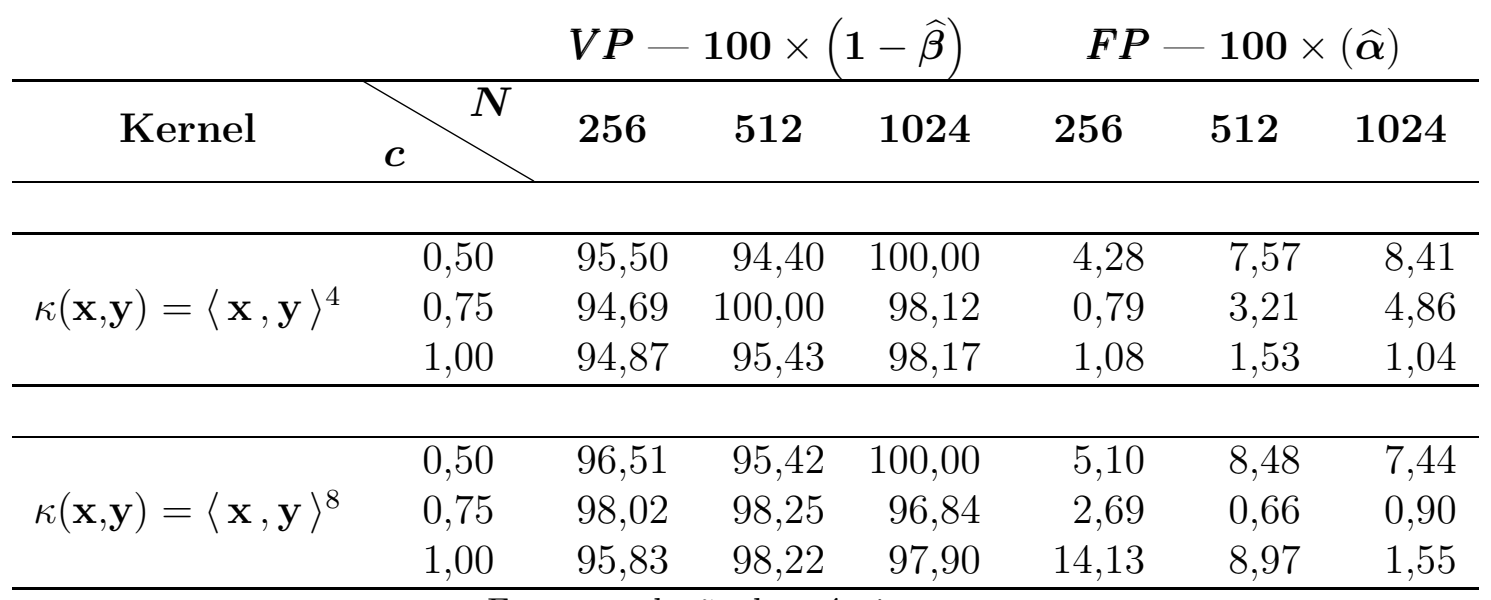

Fonte: produção do próprio autor.

Tabela 9 - Tabela de contingência para o exemplo 3 , referente ao $k n$ DTF.

\begin{tabular}{|c|c|c|c|c|c|c|c|}
\hline & & $V P$ & $100 \times$ & $-\widehat{\boldsymbol{\beta}})$ & $F P$ & 100 & $(\widehat{\alpha})$ \\
\hline Kernel & & 256 & 512 & 1024 & 256 & 512 & 1024 \\
\hline & 0,50 & 95,50 & 94,40 & 100,00 & 4,44 & 7,68 & 8,41 \\
\hline$\kappa(\mathbf{x}, \mathbf{y})=\langle\mathbf{x}, \mathbf{y}\rangle^{4}$ & 0,75 & 94,71 & 100,00 & 98,12 & 0,81 & 3,21 & 4,86 \\
\hline & 1,00 & 94,87 & 95,43 & 95,36 & 1,16 & 1,53 & 1,04 \\
\hline & 0,50 & 93,79 & 95,42 & 100,00 & 3,91 & 8,41 & 7,37 \\
\hline$\kappa(\mathbf{x}, \mathbf{y})=\langle\mathbf{x}, \mathbf{y}\rangle^{8}$ & 0,75 & 95,41 & 98,25 & 96,84 & 0,74 & 0,61 & 0,83 \\
\hline & 1,00 & 93,13 & 98,21 & 97,29 & 0,02 & 3,30 & 0,00 \\
\hline
\end{tabular}




\subsubsection{Exemplo 4: Modelo não-linear de banda larga 3D}

Considere o modelo composto por três variáveis, associado com as equações seguintes, ou seja,

$$
\left\{\begin{array}{l}
x_{1}(n)=3,4 x_{1}(n-1)\left[1-x_{1}^{2}(n-1)\right] \mathrm{e}^{-x_{1}^{2}(n-1)}+w_{1}(n) \\
x_{2}(n)=3,4 x_{2}(n-1)\left[1-x_{2}^{2}(n-1)\right] \mathrm{e}^{-x_{2}^{2}(n-1)}+0,35 x_{1}(n-1) x_{2}(n-1)+w_{2}(n) \\
x_{3}(n)=3,4 x_{3}(n-1)\left[1-x_{3}^{2}(n-1)\right] \mathrm{e}^{-x_{3}^{2}(n-1)}+x_{2}(n-1)+0,75 x_{1}^{2}(n-1)+w_{3}(n)
\end{array}\right.
$$

em que os processos $\left\{w_{i}(n)\right\}_{n \in \mathbb{Z}}$, novamente, são as inovações, com estatística $\mathbf{N}(0,1)$. Uma realização típica está presente na Figura 23, para $N=1024$ pontos.

Note que esse mapa é uma modificação de (3.5) e também está presente em [66] $]^{1}$.

Repare que, devido ao termo exponencial, há um espalhamento em frequência, gerando sinais em banda larga e também não-linearidades, o que é desejável para a análise. O diagrama de conectividade e de alcançabilidade está presente na Figura 22.

Figura 22 - Padrão de conectividade e alcançabilidade do exemplo 4.

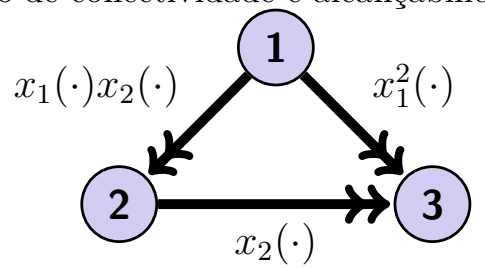

Fonte: produção do próprio autor.

Assim como nos exemplos anteriores, este apresenta acoplamentos não-lineares, da forma polinomial par, ou seja, forma quadrática $\left(x_{1}^{2}(\cdot)\right)$, além de bilinearidade $\left(x_{1}(\cdot) x_{2}(\cdot)\right)$. Estas, como já foram ditas, a PDC e a DTF não conseguem capturar, mas a conexão linear $2 \rightarrow 3$ não é problema para esses dois descritores, como confirmado adiante.

Portanto, observando as Figuras 24 e 25 referente aos quantificadores causais lineares, confirma-se que são capazes de somente identificar a conexão com acoplamento linear, como esperado.

Por outro lado, as Figuras 26 e 27 revelam que os descritores de conectividade kernelizados, para o kernel $\kappa(\mathbf{x}, \mathbf{y})=\langle\mathbf{x}, \mathbf{y}\rangle^{2}$ são capazes de desvendar todas as verdadeiras e corretas conexões. Isso não é surpresa, uma vez que, na Seção 3.3.1 foi vista a capacidade dos kernelizados de revelar conexões para kernels diferentes dos lineares.

Essas afirmações são confirmadas em mais detalhes nas Tabelas 10, 11 e 12, em que se faz um quadro comparativo entre dois kernels monomiais de ordens par (dois e quatro).

$\overline{1}$ Em [66], os autores consideram que as inovações são Gaussianas, de média nula e variância 0,015, aproximadamente e os coeficientes de acoplamento entre as séries também diferem. Aqui, considera-se que a variância das inovações são unitárias, sem perda de generalidade. 


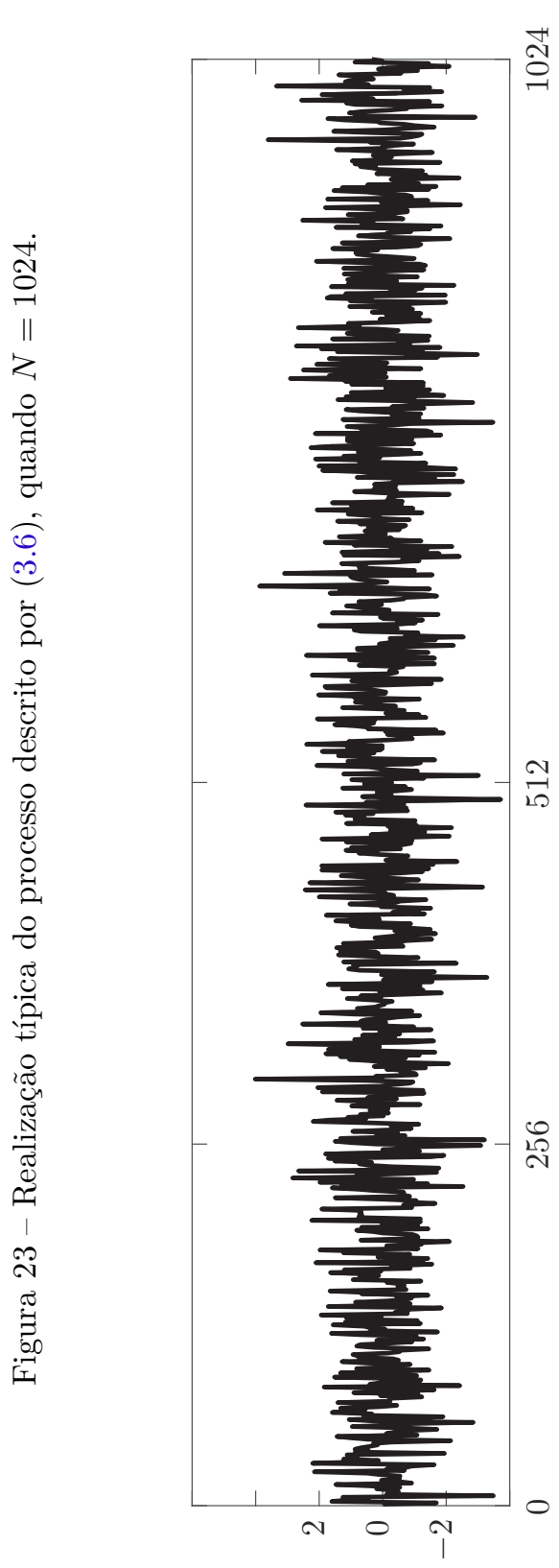

$(u) \operatorname{I} x$

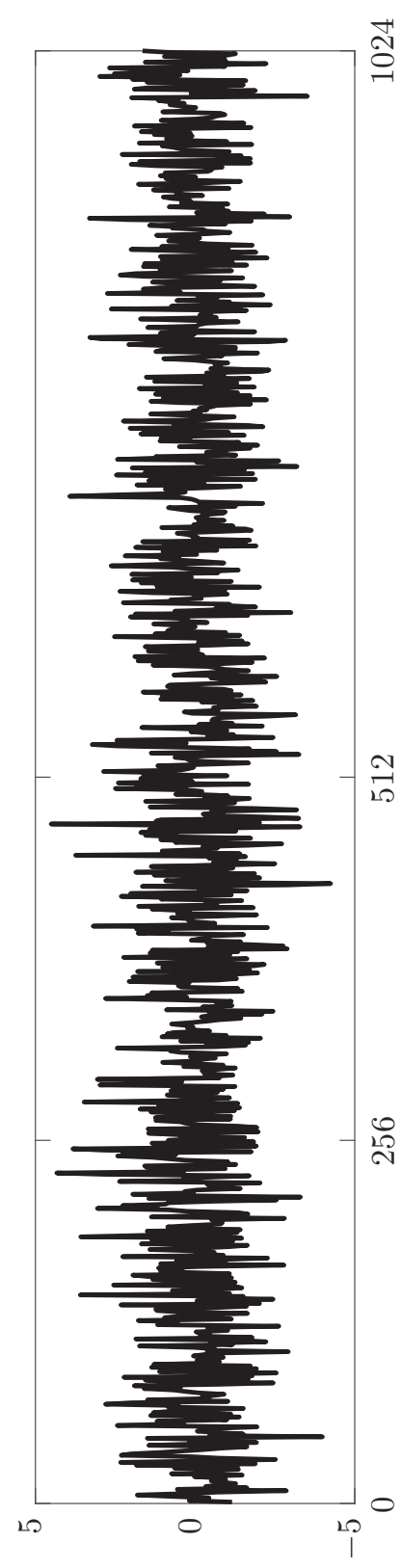

$(u)^{2} x$

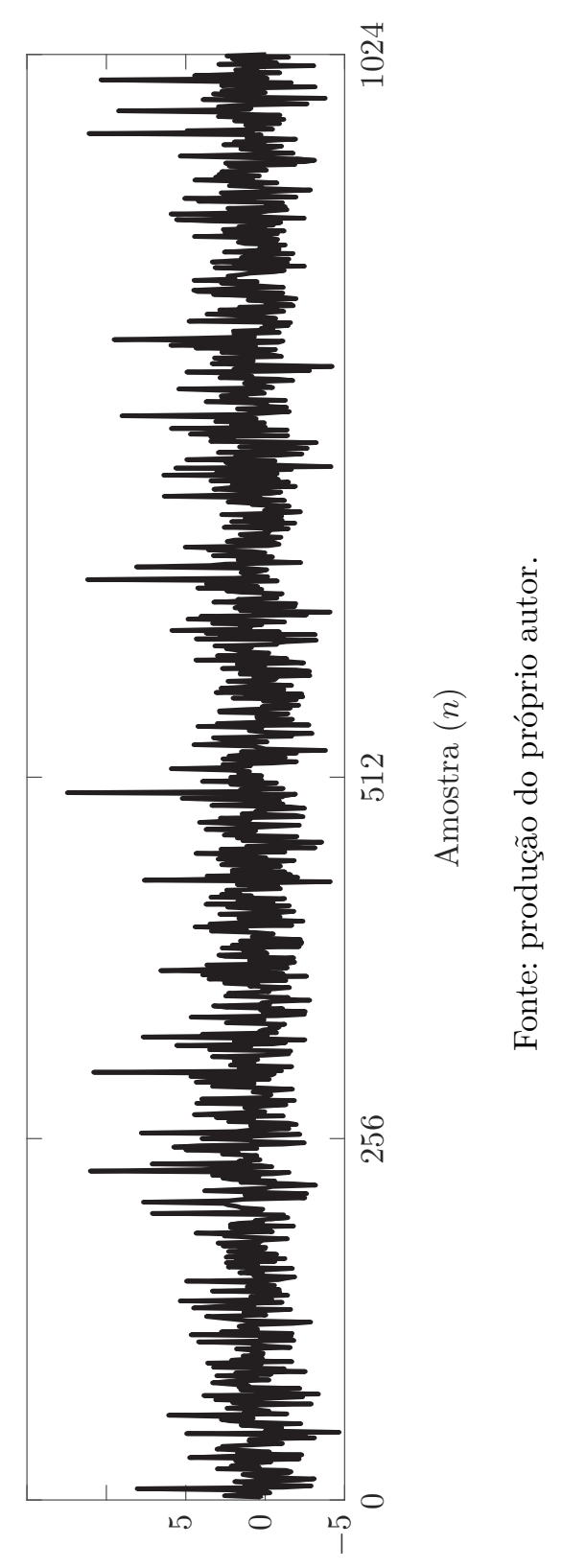

$(u)^{\varepsilon x}$ 
Figura 24 - $i$ PDC para uma realização de (3.6), considerando um nível de significância de $\alpha=0,01$. Para a escolha da ordem do modelo, utilizou-se o critério HQ, que gerou um VAR(1).
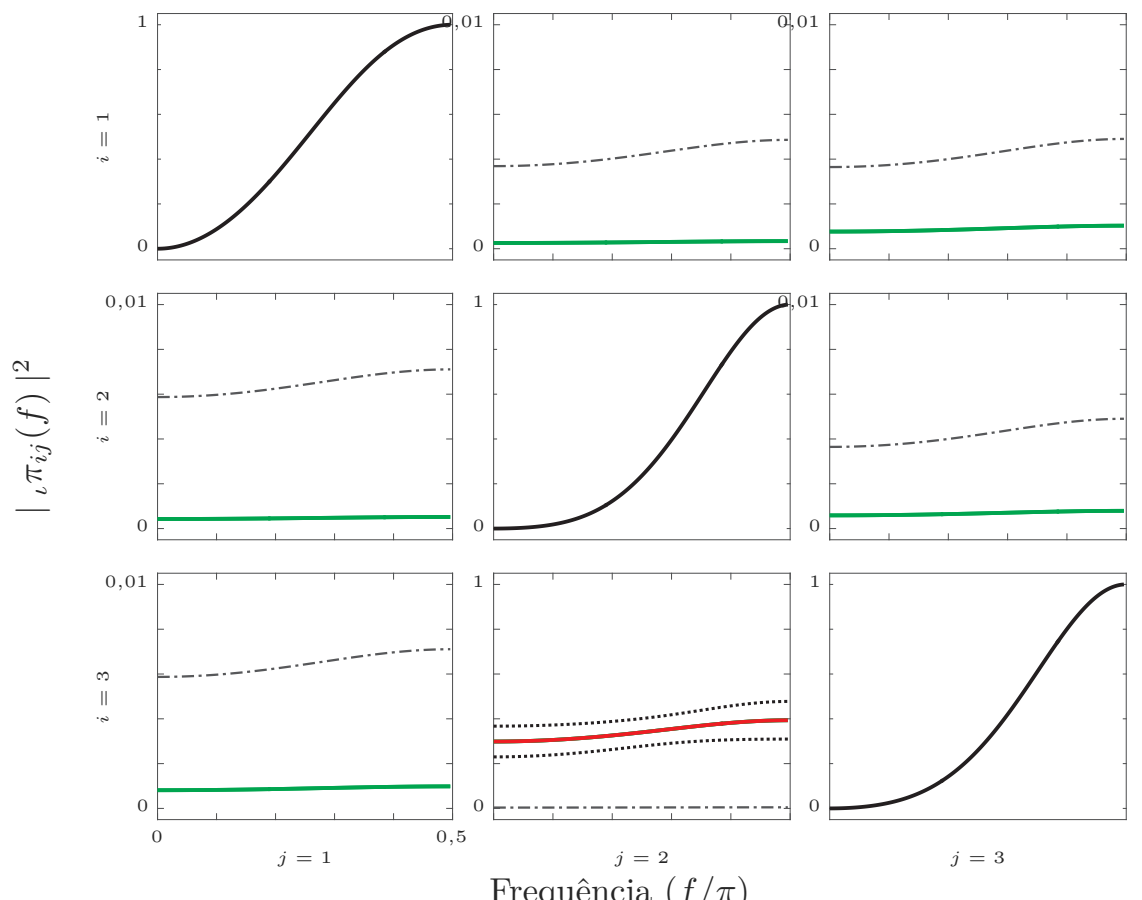

Fonte: produção do próprio autor.

Figura 25 - $i$ DTF para uma realização de (3.6), considerando um nível de significância de $\alpha=0,01$. Para a escolha da ordem do modelo, utilizou-se o critério HQ, que gerou um VAR(1).
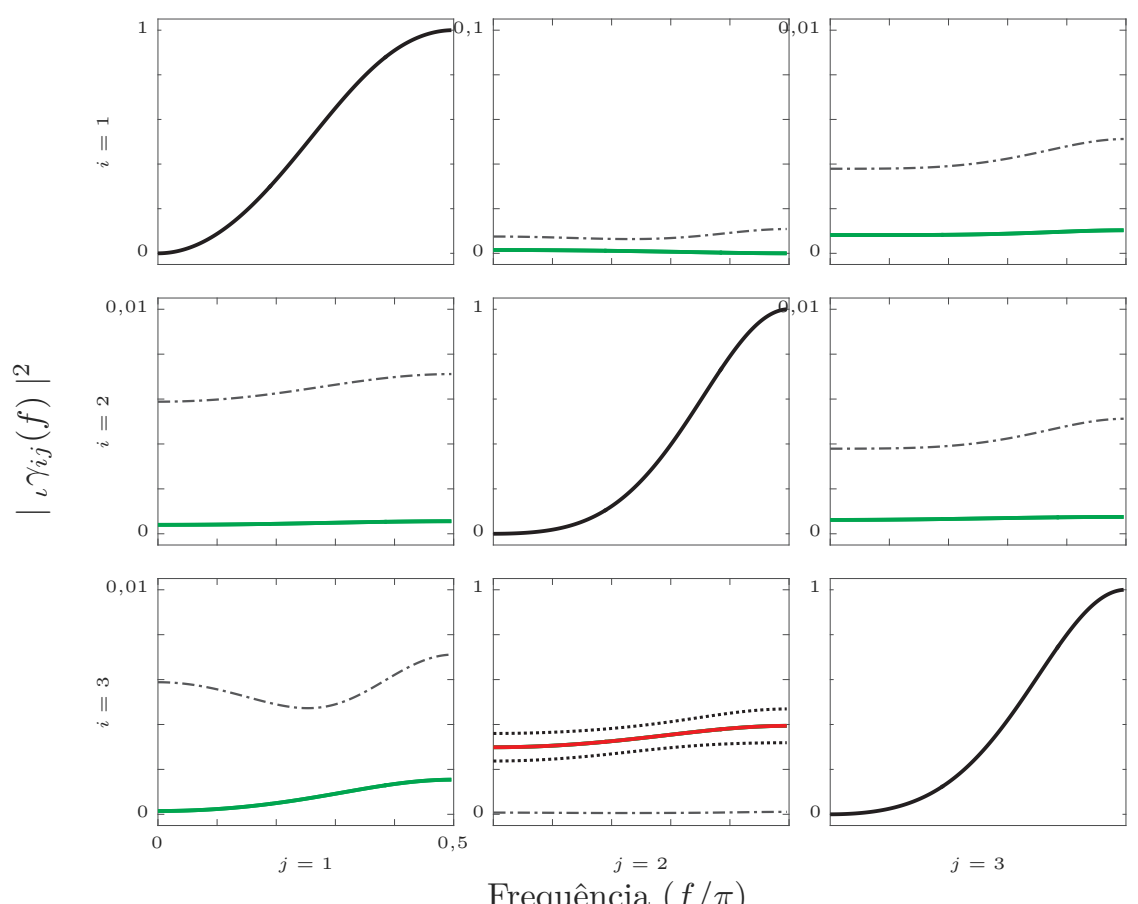

Frequência $(f / \pi)$

Fonte: produção do próprio autor. 
Figura 26 - knPDC para uma realização de (3.6), utilizando o kernel $\kappa(\mathbf{x}, \mathbf{y})=\langle\mathbf{x}, \mathbf{y}\rangle^{2}$, considerando um nível de significância de $\alpha=0,01$. Para a escolha da ordem do modelo, utilizou-se o critério $\mathrm{HQ}$, que gerou um $k \mathrm{VAR}(1)$.
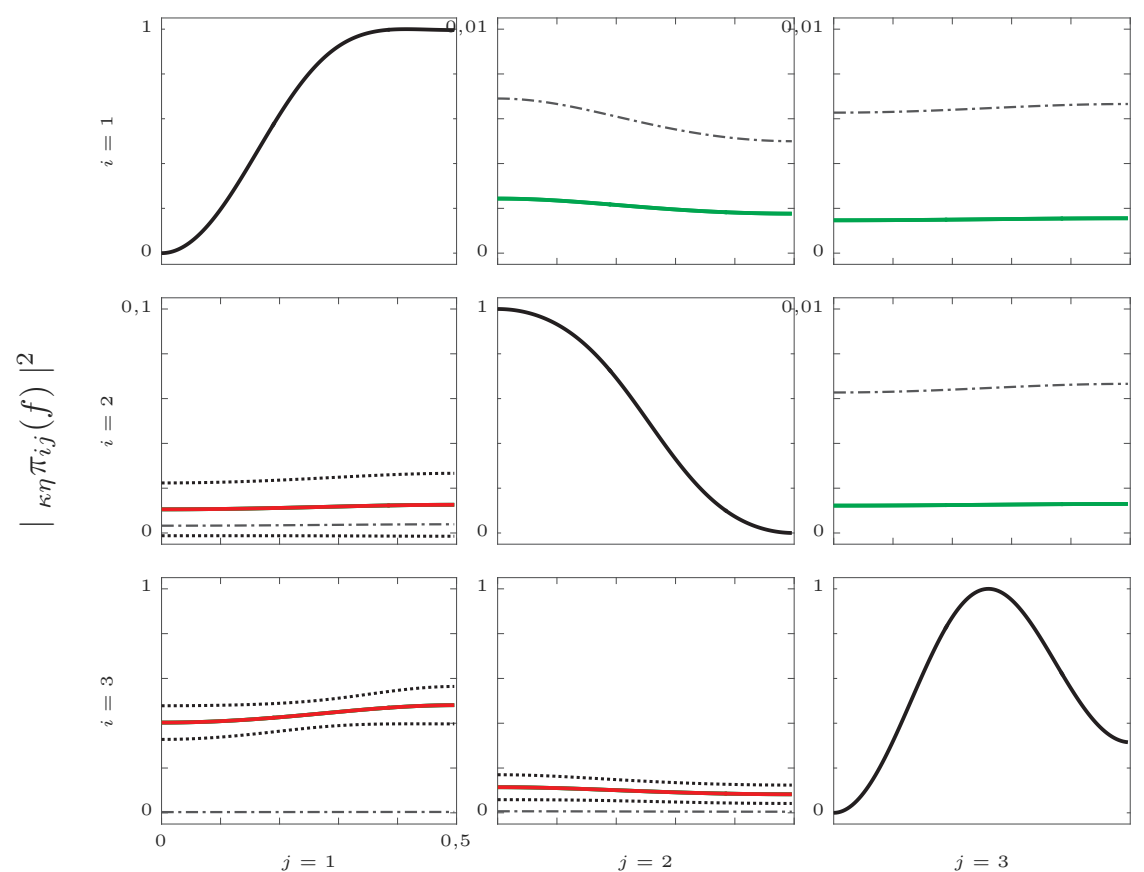

Frequência $(f / \pi)$

Fonte: produção do próprio autor.

Figura 27 - knDTF para uma realização de (3.6), utilizando o kernel $\kappa(\mathbf{x}, \mathbf{y})=\langle\mathbf{x}, \mathbf{y}\rangle^{2}$, considerando um nível de significância de $\alpha=0,01$. Para a escolha da ordem do modelo, utilizou-se o critério $\mathrm{HQ}$, que gerou um $k \operatorname{VAR}(1)$.
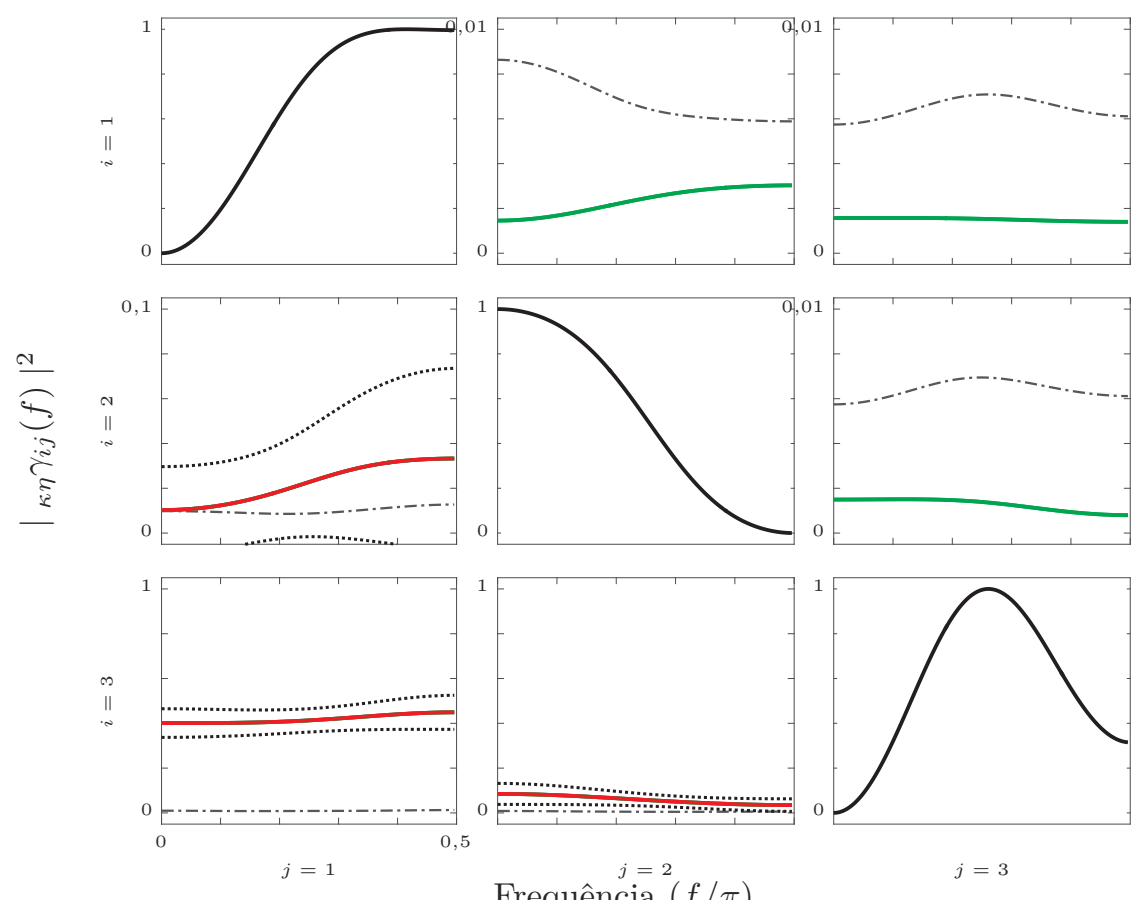

Fonte: produção do próprio autor. 
Tabela 10 - Comparação entre as taxas de verdadeiros positivos $(\boldsymbol{V P})$ e falsos positivos $(\boldsymbol{F P})$, observados, para o $k n \mathrm{GCT}\left(k n \mathrm{GCT}_{i j}\right.$, utilizando-se o kernels $\kappa_{1}(\mathbf{x}, \mathbf{y})=\langle\mathbf{x}, \mathbf{y}\rangle^{2}$ e $\left.\kappa_{2}(\mathbf{x}, \mathbf{y})=\langle\mathbf{x}, \mathbf{y}\rangle^{4}\right)$, para as conexões $i \leftarrow j$.

\begin{tabular}{|c|c|c|c|c|c|c|c|}
\hline & \multicolumn{3}{|c|}{$\kappa_{1}(\mathbf{x}, \mathbf{y})=\langle\mathbf{x}, \mathbf{y}\rangle^{2}$} & \multicolumn{3}{|c|}{$\kappa_{2}(\mathbf{x}, \mathbf{y})=\langle\mathbf{x}, \mathbf{y}\rangle^{4}$} \\
\hline & & 256 & 512 & 1024 & 256 & 512 & 1024 \\
\hline$\overline{F P}$ & $1 \leftarrow 2$ & 1,56 & 1,25 & 1,00 & 4,07 & 3,58 & 3,27 \\
\hline$V P$ & $2 \leftarrow 1$ & 44,83 & 75,21 & 96,64 & 36,85 & 57,46 & 81,77 \\
\hline$F P$ & $1 \leftarrow 3$ & 0,48 & 0,36 & 0,22 & 0,55 & 0,27 & 0,17 \\
\hline$\overline{V P}$ & $3 \leftarrow 1$ & 99,98 & 100,00 & 100,00 & 99,98 & 100,00 & 100,00 \\
\hline$\overline{F P}$ & $2 \leftarrow 3$ & 0,60 & 0,51 & 0,44 & 1,75 & 1,34 & 1,02 \\
\hline$\overline{V P}$ & $3 \leftarrow 2$ & $\overline{96,82}$ & 99,94 & 100,00 & 67,96 & 84,57 & $\overline{94,66}$ \\
\hline
\end{tabular}

Tabela 11 - Comparação entre as taxas de verdadeiros positivos $(\boldsymbol{V P})$ e falsos positivos $(\boldsymbol{F P})$, observados, para o $k n \operatorname{PDC}\left|{ }_{\kappa \eta} \pi_{i j}(f)\right|^{2}$, utilizando-se o kernels $\kappa_{1}(\mathbf{x}, \mathbf{y})=\langle\mathbf{x}, \mathbf{y}\rangle^{2}$ e $\left.\kappa_{2}(\mathbf{x}, \mathbf{y})=\langle\mathbf{x}, \mathbf{y}\rangle^{4}\right)$, para as conexões $i \leftarrow \boldsymbol{j}$.

\begin{tabular}{|c|c|c|c|c|c|c|c|}
\hline & \multicolumn{3}{|c|}{$\kappa_{1}(\mathbf{x}, \mathbf{y})=\langle\mathbf{x}, \mathbf{y}\rangle^{2}$} & \multicolumn{3}{|c|}{$\kappa_{2}(\mathbf{x}, \mathbf{y})=\langle\mathbf{x}, \mathbf{y}\rangle^{4}$} \\
\hline & & 256 & 512 & 1024 & 256 & 512 & 1024 \\
\hline$\overline{F P}$ & $1 \leftarrow 2$ & 1,61 & 1,34 & 1,12 & 4,17 & 3,81 & 4,44 \\
\hline$\overline{V P}$ & $2 \leftarrow 1$ & 45,05 & 75,36 & 96,65 & 37,18 & 57,77 & 89,07 \\
\hline$\overline{F P}$ & $1 \leftarrow 3$ & 0,50 & 0,37 & 0,25 & 0,57 & 0,31 & 0,27 \\
\hline$\overline{V P}$ & $3 \leftarrow 1$ & 99,98 & 100,00 & 100,00 & 99,98 & 100,00 & 100,00 \\
\hline$\overline{F P}$ & $2 \leftarrow 3$ & 0,65 & 0,56 & 0,46 & 1,87 & 1,36 & 1,61 \\
\hline$\overline{V P}$ & $3 \leftarrow 2$ & 96,88 & $\overline{99,94}$ & 100,00 & 68,22 & 84,67 & 94,74 \\
\hline
\end{tabular}

Tabela 12 - Comparação entre as taxas de verdadeiros positivos $(\boldsymbol{V P})$ e falsos positivos $(\boldsymbol{F} \boldsymbol{P})$, observados, para o $k n \operatorname{DTF}\left(\left.{ }_{\kappa \eta} \gamma_{i j}(f)\right|^{2}\right.$, utilizando-se o kernels $\kappa_{1}(\mathbf{x}, \mathbf{y})=\langle\mathbf{x}, \mathbf{y}\rangle^{2}$ e $\left.\kappa_{2}(\mathbf{x}, \mathbf{y})=\langle\mathbf{x}, \mathbf{y}\rangle^{4}\right)$, para as conexões $i \leftarrow \boldsymbol{j}$.

\begin{tabular}{|c|c|c|c|c|c|c|c|}
\hline & \multicolumn{3}{|c|}{$\kappa_{1}(\mathbf{x}, \mathbf{y})=\langle\mathbf{x}, \mathbf{y}\rangle^{2}$} & \multicolumn{3}{|c|}{$\kappa_{2}(\mathbf{x}, \mathbf{y})=\langle\mathbf{x}, \mathbf{y}\rangle^{4}$} \\
\hline & & 256 & 512 & 1024 & 256 & 512 & 1024 \\
\hline$\overline{F P}$ & $1 \leftarrow 2$ & 1,80 & 1,64 & 1,37 & 4,16 & 3,78 & 3,39 \\
\hline$\overline{V P}$ & $2 \leftarrow 1$ & 43,74 & 74,32 & 96,28 & 34,34 & 54,87 & 80,51 \\
\hline$F P$ & $1 \leftarrow 3$ & 0,78 & 0,62 & 0,34 & 0,85 & 0,49 & 0,29 \\
\hline$V P$ & $3 \leftarrow 1$ & 99,99 & 100,00 & 100,00 & 99,95 & 100,00 & 100,00 \\
\hline$F P$ & $2 \leftarrow 3$ & 0,77 & 0,65 & 0,49 & 1,85 & 1,39 & 1,04 \\
\hline$\overline{V P}$ & $3 \leftarrow 2$ & 96,32 & $\overline{99,89}$ & 100,00 & 64,54 & 82,94 & $\overline{94,27}$ \\
\hline
\end{tabular}




\subsection{SUMÁRIO}

Nesse capítulo analisaram-se estatisticamente os métodos propostos no Capítulo 2, através de extensivas simulações de Monte Carlo de três modelos não-lineares e um lineares. A utilidade do último encontra-se no fato de que se mostrou (empiricamente) que os métodos, aqui kernelizados, são equivalentes aos lineares, mas não recomenda-se a troca de um pelo outro - neste caso - devido ao alto custo computacional. Já nos últimos três casos, fica claro que os métodos (novos) kernelizados são capazes de identificar a correta direção de causalidade, mas ficou claro ser necessário ter um bom conhecimento prévio da estrutura do mapa (sistema) que se trabalha, a fim de se obter sucesso na escolha do kernel e na análise.

No próximo capítulo, será dado um fechamento à tese, assim como um panorama geral com sugestões de trabalhos futuros. 


\section{CONCLUSÕES E DISCUSSÃO}

Esse trabalho considerou a inferência exploratória do efeito de não-linearidades na interação entre em séries temporais vetoriais. Durante o estudo foram examinadas diversas técnicas, porém optou-se por fazer mapeamento não-linear das séries utilizandose conceitos como a Regressão Kernelizada, os chamados Métodos de Kernel.

Revisou-se o tema quanto às formas de modelos que pudessem ser utilizados. Em fase posterior, fez-se um detalhamento da teoria de kernels o que permitiu aplicar modelos lineares $\operatorname{VAR}(p)$ para sistemas não-lineares, representando-os de forma kernelizada com o auxílio do kernel trick. Isso exigiu que as medidas de fluxo de informação também sofressem a transformação de kernel, ou seja, foram propostos os estimadores $k n \mathrm{GCT}$, $k n \mathrm{DTF}$ e $k n \mathrm{PDC}$. Outro resultado importante obtido foram as estatísticas assintóticas dos novos estimadores citados anteriormente, que, novamente, foram possíveis graças ao kernel trick.

Em seguida, descreveu-se o uso dos métodos propostos, aplicados à detecção de causalidade em quatro exemplos. Em uma primeira análise, as técnicas mostram-se atraentes, pois são capazes de capturar a correta direção de causalidade no caso de interação não-linear. Porém investigações sugerem que uma escolha criteriosa do kernel é crucial (como pode ser vista nas tabelas de contingência das Seções 3.3.1, 3.3.2, 3.3.3 e 3.3.4) e cuja determinação requer técnicas poli-espectrais padrão [73]. Outras duas observações importantes são: (i) dados discrepantes (outliers) exigem o uso de algoritmos robustos no espaço de características, sendo uma consideração prática penosa e (ii) a detectabilidade diminui conforme o modelo aumenta em ordem e número de séries simultâneas analisadas.

Dadas as informações acima, através de análise de sensibilidade e tabelas de contingência, mostra-se por simulações de Monte Carlo que a nova metodologia é capaz de inferir corretamente a direção de causalidade.

Note-se também, que em alguns casos são necessários pré-processamentos cuidadosos para retirada de uma certa frequência de interesse [74], ou mesmo, se existir, a dependência de longa duração das autocorrelações [69,70], pois esses fenômenos podem mascarar a correta captura da causalidade, mesmo em casos lineares.

Como uma questão de simulação, vale a pena mencionar que os resultados relatados no texto foram obtidos com MATLAB, na versão R2013a. Notou-se que em versões diferentes (às anteriores), houve um comprometimento importante na análise dos resultados, alterando o comportamento estatístico observado. Isso se deve a melhorias que a empresa MathWorks [75] está incorporando ao tratamento numérico das matrizes, como, por exemplo, questões relativas aos diversos algoritmos de Álgebra Linear numérica, referentes a 
matrizes, incluindo os de inversão, multiplicação, decomposições espectrais, normas etc, fazendo com que, nas versões mais atuais, haja uma maior estabilidade numérica.

As observações anteriores sugerem uma continuidade da pesquisa na área antes que a metodologia possa ser posta em prática, já que uma metodologia de escolha sistemática de kernel, associada a um bom ajuste dos dados no espaço de características, necessita ser desenvolvida. Entretanto, teoricamente, é necessário esmiuçar uma interpretação quantitativa e qualitativa correta dos resultados, uma vez que quantificadores, como $k n \mathrm{PDC}$ e $k n \mathrm{DTF}$, são essencialmente decomposições espectrais de processos no espaço de características.

Finalmente, tendo por base os estudos de caso apresentados quando os métodos lineares se mostram incapazes de reconstruir corretamente a conectividade original, é possível concluir que as técnicas propostas aqui abrem uma abordagem viável para detectar Causalidade de Granger nos domínios do tempo e da frequência.

\subsection{TRABALHOS FUTUROS}

Como foi sugerido no Capítulo 1, esse trabalho poderia gerar o estudo do problema de obtenção ótima de kernel, assim como dos seus parâmetros, com base nos dados disponíveis. Outra possibilidade de estudo é explorar a metodologia em dimensões maiores, já que aqui são contemplados apenas casos de duas e três dimensões, de banda-estreita (com alta ressonância) e banda-larga, com realimentações polinomiais quadráticas e, em um caso, bilinear. Em aplicações de neurociências e outras da biomedicina seria necessário considerar o efeito para mapas mais gerais e um número maior de sinais simultâneos $(D \ggg 2)$.

Ainda, quando se trata de problemas de grandes dimensões, observa-se que a matriz de kernel aumenta excessivamente, lembrando que a dimensão desta é $D N \times D N$, com $D$ sendo o número de séries (ou canais) simultâneos analisados e $N$ o número de amostras temporais. Portanto é necessário o estudo de considerações práticas, tais como: (i) redução de dimensionalidade (como a Fatoração Incompleta de Cholesky [16,76]) para tratamento numérico da matriz de kernel ou, ainda, (ii) regularização da matriz de kernel. Os dois estudos são necessários, pois a matriz obtida no contexto do método mal-condicionada, tornando-a quase singular. Isso exige que se estude mais a fundo o problema da regularização para uso em Métodos de Kernel. 


\section{REFERÊNCIAS}

1 BROCKWELL, P. J.; DAVIS, R. A. Introduction to time series and forecasting. 2nd. ed. New York: Springer, 2002. (Springer texts in statistics).

2 BROCKWELL, P. J.; DAVIS, R. A. Time series: Theory and methods. 2nd. ed. New York: Springer-Verlag, 1991. (Springer series in statistics).

3 HAMILTON, J. D. Time series analysis. Princeton, N.J.: Princeton University Press, 1994.

4 PRIESTLEY, M. B. Spectral analysis and time series. London: Academic Press, 1981.

5 SHUMWAY, R. H.; STOFFER, D. S. Time series analysis and its applications: With $R$ examples. 3rd. ed. New York: Springer, 2010. 596 p. (Springer texts in statistics).

6 TSAY, R. S. Analysis of financial time series. 3rd. ed. Hoboken, N.J.: Wiley, 2010.

7 BACCALÁ, L. A.; SAMESHIMA, K. Partial directed coherence: A new concept in neural structure determination. Biological Cybernetics, v. 84, n. 6, p. 463-474, mar. 2001.

8 KAMIŃSKI, M. J.; CIESLAK-BLINOWSKA, K. J. A new method of the description of the information flow in the brain structures. Biological Cybernetics, Springer Berlin / Heidelberg, v. 65, n. 3, p. 203-210, fev. 1991. ISSN 0340-1200.

9 VALDES-SOSA, P. A. et al. Effective connectivity: Influence, causality and biophysical modeling. NeuroImage, v. 58, n. 2, p. 339-361, set. 2011. ISSN 1053-8119.

10 LÜTKEPOHL, H. New introduction to multiple time series analysis. Berlin: Springer, 2005 .

11 BACCALÁ, L. A.; SAMESHIMA, K. Overcoming the limitations of correlation analysis for many simultaneously processed neural structures. Progress in Brain Research, v. 130, n. Advances in Neural Population Coding, p. 33-47, jan. 2001.

12 MARMARELIS, V. Z. Nonlinear dynamic modeling of physiological systems. Piscataway, NJ: Wiley-IEEE Press, 2004. Hardcover. (IEEE Press Series on Biomedical Engineering). ISBN 0471469602.

13 MASSAROPPE, L. Caracterização da conectividade entre regiões cerebrais via entropia aproximada e causalidade de Granger. Dissertação (Dissertação de Mestrado) Escola Politécnica da Universidade de São Paulo, São Paulo, ago. 2011.

14 MASSAROPPE, L.; BACCALÁ, L. A.; SAMESHIMA, K. Semiparametric detection of nonlinear causal coupling using partial directed coherence. In: 2011 Annual International Conference of the IEEE Engineering in Medicine and Biology Society. Boston: IEEE, 2011. p. 5927-5930. ISSN 1557-170X.

15 MASSAROPPE, L.; BACCALÁ, L. A. Método semi-paramétrico para inferência de conectividade não-linear entre séries temporais. In: Anais do I Congresso de Matemática e Computacional da Região Sudeste, I CMAC Sudeste. Uberlândia: [s.n.], 2011. p. 293-296. ISSN 2237-7166. 
16 PRÍNCIPE, J. C. Information theoretic learning: Rényi's entropy and kernel perspectives. 1st. ed. New York: Springer Publishing Company, Incorporated, 2010. 448 p. (Information Science and Statistics, XIV).

17 PARK, I.; PRÍNCIPE, J. C. Correntropy based Granger causality. In: IEEE International Conference on Acoustics, Speech and Signal Processing, 2008. ICASSP 2008. Las Vegas: IEEE, 2008. p. 3605-3608. ISSN 1520-6149.

18 GENÇAY, R.; SELÇUK, F.; WHITCHER, B. An introduction to wavelets and other filtering methods in finance and economics. San Diego, California: Academic Press, 2002.

19 SETH, S.; PRÍNCIPE, J. C. A conditional distribution function based approach to design nonparametric tests of independence and conditional independence. In: Proceedings of the IEEE International Conference on Acoustics, Speech and Signal Processing (ICASSP). Dallas: IEEE, 2010. p. 2066-2069.

20 SETH, S.; PRÍNCIPE, J. C. A test of Granger non-causality based on nonparametric conditional independence. In: Proceedings of the International Conference on Pattern Recognition (ICPR). Istanbul, Turkey: IEEE, 2010. p. 2620-2623.

21 SETH, S.; PRÍNCIPE, J. C. Assessing Granger non-causality using nonparametric measure of conditional independence. IEEE Transactions on Neural Networks and Learning Systems, v. 23, n. 1, p. 47-59, jan. 2012. ISSN 2162-237X.

22 BACCALÁ, L. A.; TAKAHASHI, D. Y.; SAMESHIMA, K. Consolidating a link centered neural connectivity framework with Directed Transfer Function asymptotics. arXiv - Quantitative Biology, v. 1, p. 1-12, jan. 2015.

23 BACCALÁ, L. A. et al. Unified asymptotic theory for all partial directed coherence forms. Philosophical Transactions of the Royal Society A: Mathematical, Physical and Engineering Sciences, v. 371, n. 1997, p. 20120158, jul. 2013.

24 SCHELTER, B. et al. Testing for directed influences among neural signals using partial directed coherence. Journal of Neuroscience Methods, v. 152, n. 1-2, p. 210-219, nov. 2006. ISSN 0165-0270.

25 HLAVÁČKOVÁ-SCHINDLER, K. et al. Causality detection based on informationtheoretic approaches in time series analysis. Physics Reports - Review Section of Physics Letters, v. 441, n. 1, p. 1-46, mar. 2007. ISSN 03701573.

26 MARINAZZO, D.; PELliCORO, M.; STRAMAGLIA, S. Kernel method for nonlinear Granger causality. Physical Review Letters, American Physical Society, v. 100, n. 14 , p. 144103 , abr. 2008.

27 PALUŠ, M. et al. Synchronization as adjustment of information rates: Detection from bivariate time series. Physical Review E, American Physical Society, v. 63, n. 4, p. 046211, mar. 2001.

28 FAES, L.; NOLLO, G.; CHON, K. H. Linear and nonlinear parametric model identification to assess Granger causality in short-term cardiovascular interactions. Computers in Cardiology, v. 35, p. 793-796, jan. 2008. 
29 PEREDA, E.; QUIROGA, R. Q.; BHATTACHARYA, J. Nonlinear multivariate analysis of neurophysiological signals. Progress in Neurobiology, v. 77, n. 1-2, p. 1-37, nov. 2005. ISSN 0301-0082.

30 KANNAN, R.; TANGIRALA, A. K. Correntropy-based partial directed coherence for testing multivariate Granger causality in nonlinear processes. Physical Review E, American Physical Society, v. 89, n. 6, p. 062144, jun. 2014.

31 HE, F. et al. A nonlinear causality measure in the frequency domain: Nonlinear partial directed coherence with applications to EEG. Journal of Neuroscience Methods, n. 225, p. 71-80, jan. 2014. ISSN 0165-0270.

32 VAPNIK, V. N. Statistical learning theory. In: . 1st. ed. Hoboken, NJ: John Wiley and Sons, 1998. p. 736. ISBN 0471030031.

33 SCHÖLKOPF, B.; SMOLA, A. J. Learning with kernels: Support vector machines, regularization, optimization, and beyond. Cambridge, MA, USA: MIT Press, 2001. ISBN 0262194759 .

34 SCHÖLKOPF, B.; SMOLA, A. J.; MÜLLER, K.-R. Advances in kernel methods. In: SCHÖLKOPF, B.; BURGES, C. J. C.; SMOLA, A. J. (Ed.). Cambridge, MA, USA: MIT Press, 1999. cap. Kernel Principal Component Analysis, p. 327-352. ISBN 0-262-19416-3.

35 BACH, F. R.; JORDAN, M. I. Kernel independent component analysis. Journal of Machine Learning Research, JMLR.org, v. 3, p. 1-48, mar. 2003. ISSN 1532-4435.

36 KUMAR, R.; JAWAHAR, C. V. Kernel approach to autoregressive modeling. In: INDIAN INSTITUTE OF TECHNOLOGY, KANPUR. Thirteenth National Conference on Communications (NCC 2007). Kanpur, India, 2007. Disponível em: < http://cvit.iiit. ac.in/papers/ranjeeth07Kernel.pdf $>$. Acesso em: 06/10/2014.

37 KALLAS, M. et al. Kernel autoregressive models using Yule-Walker equations. Signal Processing, v. 93, n. 11, p. 3053-3061, abr. 2013. ISSN 0165-1684.

38 LIU, W.; PRÍNCIPE, J. C.; HAYKIN, S. Kernel adaptive filtering: A comprehensive introduction. Hoboken, NJ, USA: Wiley, 2010. (Wiley Series in Adaptive and Learning Systems for Signal Processing, Communications, and Control). ISBN 9780470447536.

39 ARONSZAJN, N. Theory of reproducing kernels. Transactions of the American Mathematical Society, v. 68, n. 3, p. 337-404, maio. 1950.

40 COVER, T. M. Geometrical and statistical properties of systems of linear inequalities with applications in pattern recognition. IEEE Transactions on Electronic Computers, EC-14, n. 3, p. 326-334, jun 1965. ISSN 0367-7508.

41 WILliAMSON, R. C.; SMOLA, A. J.; SCHÖLKOPF, B. Generalization performance of regularization networks and support vector machines via entropy numbers of compact operators. IEEE Transactions on Information Theory, v. 47, n. 6, p. 2516-2532, set. 2001. ISSN 0018-9448.

42 MERCER, J. Functions of positive and negative type, and their connection with the theory of integral equations. Philosophical Transactions of the Royal Society A: Mathematical, Physical and Engineering Sciences, A, n. 209, p. 415-446, jan. 1909. 
43 SMOLA, A. J.; SCHöLKOPF, B. A tutorial on support vector regression. Statistics and Computing, Kluwer Academic Publishers, Hingham, MA, USA, v. 14, n. 3, p. 199-222, ago. 2004. ISSN 0960-3174.

44 STEINWART, I. On the influence of the kernel on the consistency of support vector machines. Journal of Machine Learning Research, v. 2, p. 67-93, nov. 2001.

45 KIMELDORF, G. Some applications on Tchebycheffian spline functions. Journal of Mathematical Analysis and Applications, v. 33, n. 1, p. 82-95, jan. 1971.

46 SCHÖLKOPF, B.; HERBRICH, R.; SMOLA, A. J. A generalized representer theorem. In: Proceedings of the 14th Annual Conference on Computational Learning Theory and 5th European Conference on Computational Learning Theory (COLT '01/EuroCOLT '01). London, UK: Springer-Verlag, 2001. p. 416-426.

47 BOYD, S.; VANDENBERGHE, L. Convex Optimization. New York, NY, USA: Cambridge University Press, 2004. ISBN 0521833787.

48 TSANG, I. W.-H.; KWOK, J. T.-Y. Efficient hyperkernel learning using second-order cone programming. In: Machine Learning: ECML 2004. Pisa, Italy: Springer Berlin Heidelberg, 2004. (Lecture Notes in Computer Science, v. 3201), p. 453-464.

49 TSANG, I. W.-H.; KWOK, J. T.-Y. Efficient hyperkernel learning using second-order cone programming. IEEE Transactions on Neural Networks, v. 17, n. 1, p. 48-58, jan. 2006. ISSN 1045-9227.

50 ONG, C. S.; SMOLA, A. J.; WILliAMSON, R. C. Learning the kernel with hyperkernels. Journal of Machine Learning Research, n. 6, p. 1043-1071, maio. 2005.

51 GRANGER, C. W. J. Investigating causal relations by econometric models and cross-spectral methods. Econometrica, The Econometric Society, v. 37, n. 3, p. 424-438, jul. 1969. ISSN 00129682.

52 BACCALÁ, L. A.; SAMESHIMA, K. Methods in brain connectivity inference through multivariate time series analysis. In: [S.l.]: CRC Press, Boca Raton, 2014. (Frontiers in Neuroengineering Series), cap. Multivariate time series brain connectivity: A sum up, p. 245-251.

53 BACCALÁ, L. A.; SAMESHIMA, K. Causality and influentiability: The need for distinct neural connectivity concepts. In: Brain Informatics and Health. Warsaw, Poland: Springer International Publishing, 2014. (Lecture Notes in Computer Science, v. 8609), p. $424-435$.

54 PARZEN, E. Statistical inference on time series by Hilbert space method, I. Stanford, 1959.

55 MASSAROPPE, L.; BACCALÁ, L. A. Detecting nonlinear Granger causality via the kernelization of partial directed coherence. In: Proceedings of the 60th World Statistics Congress of the International Statistical Institute, ISI2015. Rio de Janeiro, RJ: International Statistical Institute, The Hague, The Netherlands, 2015. p. 2036-2041. 
56 MASSAROPPE, L.; BACCALÁ, L. A. Kernel-nonlinear-PDC extends Partial Directed Coherence to detecting nonlinear causal coupling. In: 2015 37th Annual International Conference of the IEEE Engineering in Medicine and Biology Society (EMBC). Milan: IEEE, 2015. p. 2864-2867.

57 MARDIA, K. V.; KENT, J. T.; BIBBY, J. M. Multivariate analysis. London: Academic Press, 1980. (Probability and Mathematical Statistics).

58 HABLE, R. Asymptotic normality of support vector machine variants and other regularized kernel methods. Journal of Multivariate Analysis, v. 106, p. 92-117, abr. 2012. ISSN 0047-259X.

59 MARPLE, J. S. L. Digital spectral analysis: With aplications. Upper Saddle River, NJ: Prentice Hall, 1987. 492 p. (Prentice Hall Signal Processing Series).

60 PERCIVAL, D. B.; WALDEN, A. T. Spectral analysis for physical applications: Multitaper and conventional univariate techniques. New York: Cambridge University Press, 1993. 611 p.

61 ASIMAKOPOUlOS, I.; AYLING, D.; MAHMOOD, W. M. Non-linear Granger causality in the currency futures returns. Economics Letters, v. 68, n. 1, p. 25-30, jul. 2000. ISSN 0165-1765.

62 LESAGE, J. P.; PACE, R. K. Introduction to spatial econometrics. Boca Raton, London, New York: CRC Press/Taylor \& Francis Group, 2009. 374 p. (Statistics: A Series of Textbooks and Monographs).

63 BACCALÁ, L. A.; SAMESHIMA, K. Methods in brain connectivity inference through multivariate time series analysis. In: [S.l.]: CRC Press, Boca Raton, 2014. (Frontiers in Neuroengineering Series), cap. Partial Directed Coherence, p. 57-73.

64 TAKAHASHI, D. Y.; BACCALÁ, L. A.; SAMESHIMA, K. Information theoretic interpretation of frequency domain connectivity measures. Biological Cybernetics, Springer-Verlag, v. 103, n. 6, p. 463-469, dec. 2010. ISSN 0340-1200.

65 CHEN, Y. et al. Analyzing multiple nonlinear time series with extended Granger causality. Physics Letters A, v. 324, n. 1, p. 26-35, abr. 2004. ISSN 0375-9601.

66 GOURÉVITCH, B.; BOUQUIN-JEANNÈS, R. L.; FAUCON, G. Linear and nonlinear causality between signals: Methods, examples and neurophysiological applications. Biological Cybernetics, v. 95, n. 4, p. 349-369, out. 2006. ISSN 1432-0770.

67 SAMESHIMA, K.; TAKAHASHI, D. Y.; BACCALÁ, L. A. On the statistical performance of Granger-causal connectivity estimators. Brain Informatics, v. 2, n. 2, p. 119-133, abr. 2015. ISSN 2198-4018, 2198-4026.

68 LO, A. W. Long-term memory in stock market prices. Econometrica, v. 59, n. 5, p. 1279-1313, set. 1991.

69 MASSAROPPE, L.; CADUdA, F.; BACCALÁ, L. A. On the performance of wavelet-based long-memory parameter estimation. In: ASSOCIAÇÃO BRASILEIRA DE ESTATÍSTICA (ABE). 21o. SINAPE - Simpósio Nacional de Probabilidade e Estatística. Natal, RN: ABE, 2014. 
70 MASSAROPPE, L.; BACCALÁ, L. A. A simulation comparison between fractional differencing exponent estimators. In: ASSOCIAÇÃO BRASILEIRA DE ESTATÍSTICA (ABE). XVI Escola de Séries Temporais e Econometria. Campos do Jordão, SP: ABE, 2015.

71 PERCIVAL, D. B.; WALDEN, A. T. Wavelet methods for time series analysis. Cambridge: Cambridge University Press, 2000. 594 p. (Cambridge Series in Statistical and Probabilistic Mathematics).

72 WHITCHER, B. Wavelet-based estimation for seasonal long-memory processes. Technometrics, v. 46, n. 2, p. 225-238, jan. 2004.

73 NIKIAS, C.; PETROPULU, A. P. Higher order spectra analysis: A non-linear signal processing framework. Upper Saddle River, NJ: Prentice Hall, 1993. 528 p. (Prentice Hall Signal Processing Series).

74 BACCALÁ, L. A.; SAMESHIMA, K. On neural connectivity estimation problems. In: 2015 37th Annual International Conference of the IEEE Engineering in Medicine and Biology Society (EMBC). Milan, Italy: [s.n.], 2015. p. 5400-5403. ISSN 1557-170X.

75 MATHWORKS. What's New: Learn about new product capabilities. 2016. Website. Disponível em: <http://www.mathworks.com/products/matlab/whatsnew.html>. Acesso em: 15/04/2016.

76 SETH, S.; PRÍNCIPE, J. C. On speeding up computation in information theoretic learning. In: 2009 International Joint Conference on Neural Networks. [S.l.: s.n.], 2009. p. 2883-2887. ISSN 2161-4393.

77 TIKHONOV, A. N. Solution of incorrectly formulated problems and the regularization method. In: Soviet Mathematics Doklady. [S.l.: s.n.], 1963. v. 4, p. $1035-1038$.

78 HANSEN, P. C. Rank-deficient and discrete ill-posed problems: Numerical aspects of linear inversion. Philadelphia: Society for Industrial an Applied Mathematics (SIAM), 1998. (Monographs on Mathematical Modeling an Computation).

79 SAUNDERS, C.; GAMMERMAN, A.; VOVK, V. Ridge regression learning algorithm in dual variables. In: Proceedings of the Fifteenth International Conference on Machine Learning. San Francisco, CA: Morgan Kaufmann Publishers Inc., 1998. (ICML'98), p. 515-521. ISBN 1-55860-556-8.

80 EVGENIOU, T.; PONTIL, M.; POGGIO, T. Regularization networks and support vector machines. Advances in Computational Mathematics, v. 1, n. 13, p. 1-50, abr. 2000.

81 SHANNON, C. E. A mathematical theory of communication. Bell System Technical Journal, v. 27, p. 379-423/623-656, jul./out. 1948.

82 APPLEBAUM, D. Probability and information: An integrated approach. 2nd. ed. Cambridge; New York: Cambridge University Press, 2008. 273 p.

83 COVER, T. M.; THOMAS, J. A. Elements of information theory. 2nd. ed. New York: Wiley, 2006. 774 p. (Wiley series in telecommunications). 
84 RÉNYI, A. On measures of entropy and information. In: Proceedings of the 4 th Berkeley Symposium on Mathematics, Statistics and Probability. [S.l.: s.n.], 1960. p. 547-561.

85 GRASSBERGER, P.; PROCACCIA, I. Estimation of the Kolmogorov entropy from a chaotic signals. Physical Review A, American Physical Society, v. 28, n. 4, p. 2591-2593, out. 1983.

86 GREENSIDE, H. S. et al. Impracticality of a box-counting algorithm for calculating the dimensionality of strange attractors. Physical Review A, American Physical Society, v. 25 , n. 6 , p. 3453-3456, jun. 1982.

87 PARZEN, E. On estimation of a probability density function and mode. Annals of Mathematical Sattistics, v. 33, n. 3, p. 1065-1076, set. 1962.

88 PARZEN, E. Time series analysis papers. Holden-Day: [s.n.], 1967.

89 SHEATHER, S. J. Density estimation. Statistical Science, v. 19, n. 4, p. 588-597, 2004.

90 SILVERMAN, B. W. Density estimation for statistics and data analysis. New York: Chapman and Hall/CRC, 1986. Hardcover. (Chapman \& Hall/CRC Monographs on Statistics \& Applied Probability). ISBN 0412246201.

91 SHIMAZAKI, H.; SHINOMOTO, S. Kernel bandwidth optimization in spike rate estimation. Journal of Computational Neuroscience, v. 29, n. 1-2, p. 171-182, ago. 2010.

92 SANTAMARÍA, I.; POKHAREL, P. P.; PRÍNCIPE, J. C. Generalized correlation function: Definition, properties, and application to blind equalization. IEEE Transactions on Signal Processing, v. 54, n. 6, p. 2187-2197, jun. 2006. ISSN 1053-587X.

93 SCHETZEN, M. The Volterra and Wiener theories of nonlinear systems. New York: John Wiley and Sons, 1980.

94 RUGH, W. J. Nonlinear system theory: The Volterra / Wiener approach. Baltimore: The Johns Hopkins University Press, 1981.

95 ALPER, P. A consideration of the discrete Volterra series. IEEE Transactions on Automatic Control, v. 10, n. 3, p. 322-327, jul. 1965.

96 BOYD, S.; CHUA, L. O.; DESOER, C. A. Analytical foundations of Volterra series. Journal of Mathematical Control and Information, v. 1, n. 3, p. 243-282, maio. 1984.

97 HÉLIE, T.; LAROCHE, B. Computation of convergence bounds for Volterra series of linear-analytic single-input systems. IEEE Transactions on Automatic Control, v. 56, n. 9, p. 2062-2072, set. 2011. ISSN 0018-9286.

98 GOULART, J. H. de M.; BURT, P. M. S. Efficient kernel computation for Volterra filter structure evaluation. IEEE Signal Processing Letters, v. 19, n. 3, p. 135-138, mar. 2012. ISSN 1070-9908.

99 BURT, P. M. S.; GOULART, J. H. de M. Evaluating the potential of VolterraParafac IIR models. In: 2013 IEEE International Conference onAcoustics, Speech and Signal Processing (ICASSP). [S.l.: s.n.], 2013. p. 5745-5749. ISSN 1520-6149. 
100 GIRI, F.; BAI, E.-W. (Ed.). Block-oriented nonlinear system identification. Berlin: Springer-Verlag, 2010. v. 404. 431 p. (Lecture Notes in Control and Information Sciences, v. 404).

101 AGUIRRE, L. A. Introdução à indentificação de sistemas: Técnicas lineares e não-lineares aplicadas a sistemas reais. 4a. edição revista e ampliada. ed. Belo Horizonte: Editora UFMG, 2015.

102 BIAGIOLA, S.; FIGUEROA, J. Wiener and Hammerstein uncertain models identification. Mathematics and Computers in Simulation, v. 79, n. 11, p. 3296-3313, jul. 2009. ISSN 0378-4754.

103 GÓMEZ, J. C.; BAEYENS, E. Identification of block-oriented nonlinear systems using orthonormal bases. Journal of Process Control, v. 14, n. 6, p. 685-697, set. 2004. ISSN 0959-1524.

104 VANDERSTEEN, G.; ROLAIN, Y.; SCHOUKENS, J. Non-parametric estimation of the frequency-response functions of the linear blocks of a Wiener-Hammerstein model. Automatica, v. 33, n. 7, p. 1351-1355, jul. 1997. ISSN 0005-1098.

105 CAMPello, R. J.; AMARAL, W. C. do; FAVIER, G. A note on the optimal expansion of Volterra models using Laguerre functions. Automatica, v. 42, n. 4, p. 689-693, abr. 2006. ISSN 0005-1098.

106 AGUIRRE, L. A.; CORRÉA, M. V.; CASSINI, C. C. S. Nonlinearities in NARX polynomial models: Representation and estimation. IEE Proceedings - Control Theory and Applications, v. 149, n. 4, p. 343-348, jul. 2002. ISSN 1350-2379.

107 CHEN, S.; BILLINGS, S. A. Representation of non-linear systems: The NARMAX model. International Journal of Control, Taylor \& Francis, v. 49, n. 3, p. 1012-1032, mar. 1989. Address: London.

108 HARRISON, L.; PENNY, W. D.; FRISTON, K. Multivariate autoregressive modeling of fMRI time series. NeuroImage, v. 19, n. 4, p. 1477-1491, ago. 2003. ISSN 1053-8119.

109 BILLINGS, S. A. Nonlinear system identification: NARMAX methods in the time, frequency, and spatio-temporal domains. United Kingdom: Wiley, 2013. 


\section{APÊNDICE A - MÍNIMOS QUADRADOS KERNELIZADOS}

Neste Apêndice são apresentados os métodos de regressão Ridge (Ridge regression - RR) e sua versão kernelizada, regressão Ridge kernelezida (kernel Ridge regression — $\mathrm{KRR}$ ).

Assume-se conhecida a série de entrada do sistema, para que seja possível identificála em uma dada parametrização, por exemplo, autorregressiva (AR), ou não-linear autorregressiva, com entrada exógena (NARX).

\section{A.1 REGRESSÃO RIDGE}

Seja o vetor de dados do problema $\mathbf{b}$, em $\mathbf{R}^{m}$, o vetor de variáveis $\mathbf{x}$, em $\mathbb{R}^{n}$ e uma matriz $\mathbf{A}$, em $\mathbb{R}^{m \times n}$, que os relaciona linearmente, ou seja, $\mathbf{A x}=\mathbf{b}$. Assim, o problema aqui abordado, conhecido como regressão linear, visa modelar um mapeamento $y=f(\mathbf{x})$, associado a esses dados com o objetivo de se encontrar uma solução para a melhor aproximação $\mathbf{A x} \approx \mathbf{b}$, no sentindo de uma norma $\|\cdot\|_{p}$ adequada. Para isso, considera-se o vetor

$$
\mathbf{r}=\mathbf{A x}-\mathbf{b}
$$

que recebe o nome de resíduo e suas componentes são chamadas de resíduos associados a $\mathbf{x}$.

Logo, para o referido ajuste (modelamento), se for escolhido minimizar o quadrado do erro da norma-2, então recai-se no conhecido problema de minimização de mínimos quadrados, ou seja,

$$
\begin{aligned}
\min _{\mathbf{x}}\{J(\mathbf{x})\} & =\min _{\mathbf{x}}\left\{\|\mathbf{r}\|_{2}^{2}\right\} \\
& =\min _{\mathbf{x}}\left\{\|\mathbf{A} \mathbf{x}-\mathbf{b}\|_{2}^{2}\right\} .
\end{aligned}
$$

Tipicamente, tem-se que o número de observações $m$ é maior do que o de variáveis $n$, fazendo com que o sistema acima receba o nome de sobredeterminado. Como o referido problema é convexo, pode-se mostrar [47] que sempre há uma solução. Assim, basta derivar (A.2) e igualar ao vetor nulo, para se obter,

$$
\mathbf{x}=\mathbf{A}^{\dagger} \mathbf{b}=\left(\mathbf{A}^{\top} \mathbf{A}\right)^{-1} \mathbf{A}^{\top} \mathbf{b}
$$

se $\left(\mathbf{A}^{\top} \mathbf{A}\right)^{-1}$ não for singular. 
Para se contornar esse último fato, Tikhonov [77] considera o problema de minimizar, sobre os coeficientes $\mathbf{x}, J(\mathbf{x})=\|\mathbf{A} \mathbf{x}-\mathbf{b}\|_{2}^{2}+\lambda\|\mathbf{x}\|_{2}^{2}$. Portanto, deve-se ter

$$
\begin{aligned}
\min _{\mathbf{x}}\{J(\mathbf{x})\} & =\min _{\mathbf{x}}\left\{\|\mathbf{A} \mathbf{x}-\mathbf{b}\|_{2}^{2}+\lambda\|\mathbf{x}\|_{2}^{2}\right\} \\
& =\min _{\mathbf{x}}\left\{\|\widetilde{\mathbf{A}} \mathbf{x}-\widetilde{\mathbf{b}}\|_{2}^{2}\right\},
\end{aligned}
$$

sendo $\widetilde{\mathbf{A}}:=\left[\begin{array}{c}\mathbf{A} \\ \sqrt{\lambda} \mathbf{I}_{n}\end{array}\right], \widetilde{\mathbf{b}}:=\left[\begin{array}{l}\mathbf{b} \\ \mathbf{0}\end{array}\right]$ e $\lambda>0$ recebe o nome de parâmetro de regularização da regressão Ridge ${ }^{1}$. A presença da matriz identidade, na matriz $\widetilde{\mathbf{A}}$, de dimensão $((m+n) \times n)$, garante que esta tenha posto (de colunas) completo.

Logo, procedendo da mesma maneira como foi descrito para se obter (A.3), tem-se

$$
\nabla_{\mathbf{x}} J(\mathbf{x})=\mathbf{A}^{\top} \mathbf{A} \mathbf{x}-\mathbf{A}^{\top} \mathbf{b}+\lambda \mathbf{x}=\mathbf{0}
$$

e, portanto, a solução da regressão Ridge é,

$$
\mathbf{x}=\left(\widetilde{\mathbf{A}}^{\top} \widetilde{\mathbf{A}}\right)^{-1} \widetilde{\mathbf{A}}^{\top} \mathbf{b}=\left(\mathbf{A}^{\top} \mathbf{A}+\lambda \mathbf{I}_{n}\right)^{-1} \mathbf{A}^{\top} \mathbf{b}
$$

Agora, como foi posto no Capítulo 1, em vista do Teorema da Representação [45] a solução do problema (A.4) pode ser escrita como a combinação linear de parâmetros $\boldsymbol{\alpha}$ (a serem obtidos adiante), ou seja,

$$
\mathbf{x}=\mathbf{A}^{\top} \boldsymbol{\alpha}=\sum_{i=1}^{n} \alpha_{i} \mathbf{a}_{i}
$$

sendo $\boldsymbol{\alpha}$ um vetor do $\mathbb{R}^{m}$ e $\mathbf{a}_{i}$ as colunas de $\mathbf{A}$.

Agora, rescrevendo (A.5) como,

$$
\mathbf{x}=\frac{1}{\lambda} \mathbf{A}^{\top}(\mathbf{b}-\mathbf{A} \mathbf{x})
$$

e substituindo (A.7) em (A.8), tem-se que,

$$
\boldsymbol{\alpha}=\frac{1}{\lambda}(\mathbf{b}-\mathbf{A x}) .
$$

De (A.7), sabe-se que $\mathbf{x}=\mathbf{A}^{\top} \boldsymbol{\alpha}$ e, portanto, substitui-se em (A.9) para ter-se,

$$
\boldsymbol{\alpha}=\frac{1}{\lambda}\left(\mathbf{b}-\mathbf{A} \mathbf{A}^{\top} \boldsymbol{\alpha}\right)
$$

ou seja, os coeficientes $\boldsymbol{\alpha}$ da expansão do Teorema da Representação são dados por,

$$
\boldsymbol{\alpha}=\left(\mathbf{A A}^{\top}+\lambda \mathbf{I}_{m}\right)^{-1} \mathbf{b}
$$

que é a solução dual de (A.6). Note que (A.11) é baseada na matriz de Gram $\mathbf{A A}^{\top}$, que pertence a $\mathbb{R}^{m \times m}$. Essa, carrega, em suas respectivas posições, os produtos internos $\left\langle\mathbf{a}_{i}, \mathbf{a}_{j}\right\rangle$, permitindo aplicar o kernel trick e, portanto, kernelizar essa forma de regressão. Como será visto, resolver o problema dual é mais viável devido a dimensão do espaço de características ser muito maior do que o de entrada, ou seja, $m \gg n$.

\footnotetext{
1 Detalhes em como se obter o parâmetro de regularização podem ser apreciados em [78].
} 


\section{A.2 REGRESSÃO RIDGE KERNELIZADA}

Se os dados possuem relações não lineares, a técnica anterior não será capaz obter um bom modelamento. Há diferentes técnicas para se ajustar um conjunto de dados nãolinear, mas aqui será focado nos métodos de kerneis, a que se dá o nome de regressão ridge kernelizada (KRR) [79,80]. Essa é uma técnica que visa minimizar um mapeamento não linear $\mathbf{f}(\mathbf{x})$, representado por uma expansão em kerneis, através da função custo,

$$
\min _{\mathbf{f}(\mathbf{x}) \in \mathbb{H}}\{J(\mathbf{f}(\mathbf{x}))\}=\min _{\mathbf{f}(\mathbf{x}) \in \mathbb{H}}\left\{\sum_{i=1}^{m}\left(y_{i}-f\left(a_{i}\right)\right)^{2}+\lambda\left\|\mathbf{f}\left(\mathbf{x}_{i}\right)\right\|_{\mathbb{H}}^{2}\right\},
$$

em que $\mathbb{H}$ é um RKHS, associado com um certo kernel de Mercer e $\lambda$ é a constante de regularização. Logo, a KRR explora a capacidade da propriedade da aproximação universal dos métodos de kerneis, dada uma precisão qualquer.

O Teorema da Representação mostra que a função minimizadora de (A.12) é dada por,

$$
f(\cdot)=\sum_{i=1}^{m} \alpha_{i} \kappa\left(\mathbf{a}_{i}, \cdot\right)
$$

em que, como antes, $\mathbf{a}_{i}$ são as colunas de $\mathbf{A}$ e $\alpha_{i}$ são os coeficientes da expansão. Assim, substituindo (A.13) em (A.12), tem-se que,

$$
\begin{aligned}
\min _{\boldsymbol{\alpha}}\{J(\boldsymbol{\alpha})\} & =\min _{\boldsymbol{\alpha}}\left\{\sum_{i=1}^{m}\left(y_{i}-\sum_{j=1}^{m} \alpha_{i} \kappa\left(\mathbf{a}_{j}, \mathbf{a}_{i}\right)\right)^{2}+\lambda \sum_{i=1}^{m} \sum_{j=1}^{m} \alpha_{i} \alpha_{j} \kappa\left(\mathbf{a}_{i}, \mathbf{a}_{j}\right)\right\} \\
& =\min _{\boldsymbol{\alpha}}\left\{\|\mathbf{y}-\mathbf{K} \boldsymbol{\alpha}\|_{2}^{2}+\lambda \boldsymbol{\alpha}^{\top} \mathbf{K} \boldsymbol{\alpha}\right\}
\end{aligned}
$$

sendo $\boldsymbol{\alpha}=\left[\alpha_{1}, \cdots, \alpha_{m}\right]^{\top}$ e $\mathbf{K}(\mathbf{a}, \mathbf{a})$ a matriz de kernel.

Logo, a solução de (A.14) é dada por,

$$
\boldsymbol{\alpha}=\left(\mathbf{K}(\mathbf{a}, \mathbf{a})+\lambda \mathbf{I}_{m}\right)^{-1} \mathbf{y}
$$

A dedução da solução da KRR para o caso vetorial (multivariado) é analoga e é dada por,

$$
\boldsymbol{\alpha}=\left(\mathbf{K}(\mathbf{A}, \mathbf{A})+m \lambda \mathbf{I}_{m d}\right)^{-1} \mathbf{y},
$$

em que, no caso, há $d$ conjuntos de dados sendo analisados simultaneamente. Portanto, agora, $\boldsymbol{\alpha}=\operatorname{vec} \boldsymbol{\alpha}_{i}$ e $\mathbf{y}=\operatorname{vec} \mathbf{y}_{i}$ são vetores de dimensão $(m d \times 1)$ e $\mathbf{K}(\mathbf{A}, \mathbf{A})$ é uma matriz de kernel com estrutura toeplitz em blocos, de dimensão $(m d \times m d)$. 


\section{APÊNDICE B - TEORIA DA INFORMAÇÃO}

Métodos que envolvem direta ou indiretamente apenas a descrição de segunda ordem de processos estocásticos são somente capazes de representar fielmente aspectos relativos ao acoplamento linear entre séries temporais observadas [16].

Dessa limitação, surge a necessidade de buscar outras abordagens. Como, intuitivamente, o acoplamento de grandezas dinâmicas têm fundamento na idéia de que interações são essencialmente processos de troca de informação, segue a idéia de buscar elementos para generalizar tal descrição pelo uso da Teoria da Informação, cuja origem é dada pelo trabalho de Shannon [81] e ligada à Teoria de Probabilidades [82].

\section{B.1 ASPECTOS GERAIS}

Ao invés de prover uma descrição rigorosa, como a de [83], discorre-se brevemente sobre alguns aspectos gerais, ainda que qualitativos.

A idéia central da teoria da informação é associar, a eventos aleatórios, algum conceito de posse de conhecimento. Embora a definição varie conforme o objeto aleatório envolvido $O=X$ (evento, variável aleatória discreta ou continua ou, ainda, processo) ${ }^{1}$, associa-se uma quantia chamada de informação $I(E)=-\log (\mathbb{P}\{E\})$, ao valor $E$ obtido em um experimento envolvendo $O$, no sentido de que quanto maior sua probabilidade, $\mathbb{P}\{E\}$, menor a surpresa na obtenção deste valor e, portanto, maior a informação disponível sobre sua obtenção.

Mais importante do que tipificar um dado valor $E$, é básico representar as características de $O$, como um todo, com auxílio da noção de entropia que representa o valor médio da informação associada às probabilidades dos objetos $O$. Nessas condições a entropia corresponde à esperança e, portanto,

$$
H(X)=\mathbb{E}[\mathbb{P}\{X\}]
$$

definida sobre a medida de probabilidade $\mathbb{P}$ associada aos elementos que compõem $X$. Note que (B.1) é conhecida como Entropia de Shannon.

Rényi [84] buscou uma definição geral para medidas de informação, que preservassem a aditividade de eventos independentes e fossem compatíveis com os axiomas (de Kolmogorov) da probabilidade. A partir do funcional de Cauchy $f(x y)=f(x)+f(y)$, a

$\overline{1}$ Aspectos como a eventual nulidade da probabilidade ou a natureza continua dos valores de $O$ são os repensáveis por se ter que se adotar definições diferentes para a informação, em cada caso. 
entropia de Rényi foi definida como sendo,

$$
H_{\alpha}(X)= \begin{cases}\frac{1}{1-\alpha} \log \sum_{x_{i} \in X} p_{X}^{\alpha}\left(x_{i}\right) & \text { se } X \text { é discreta; } \\ \frac{1}{1-\alpha} \log \int_{\mathbb{R}} f_{X}^{\alpha}(x) \mathrm{d} x & \text { se } X \text { é contínua; }\end{cases}
$$

com $\alpha$ em $\mathbb{R}_{+}, \alpha \neq 1$ e $\left(x_{i}, p_{i}\right)$ e $f_{X}(x)$ as funções densidade de probabilidade (probability density function - pdf), nos casos discreto e contínuo, respectivamente. Note que se for tomado o limite com $\alpha \rightarrow 1$, em (B.2), através do auxílio da regra de L'Hospital, a entropia de Rényi torna-se a de Shannon.

Agora, definindo o potencial- $\alpha$ de informação como

$$
V_{\alpha}(X)=\|p\|_{\alpha}^{\alpha}= \begin{cases}\sum_{x_{i} \in X} p_{X}^{\alpha}\left(x_{i}\right), & \text { se } X \text { é discreta; } \\ \int_{\mathbb{R}} f_{X}^{\alpha}(x) \mathrm{d} x, & \text { se } X \text { é contínua }\end{cases}
$$

tem-se

$$
H_{\alpha}(X)=\frac{1}{1-\alpha} \log \left[V_{\alpha}(X)\right]=\log \left\{\left[V_{\alpha}(X)\right]^{1 /(1-\alpha)}\right\}
$$

em que $V_{\alpha}(X)$ é a norma- $\alpha$ da pdf.

Fazendo-se $\alpha=2$, em (B.2), tem-se a entropia quadrática de Rényi, ou seja,

$$
H_{2}(X)= \begin{cases}-\log \sum_{x_{i} \in X} p_{X}^{2}(x) & \text { se } X \text { é discreta; } \\ -\log \int_{\mathbb{R}} f_{X}^{2}(x) \mathrm{d} x & \text { se } X \text { é contínua. }\end{cases}
$$

Note que essa escolha faz o argumento do logaritmo, em (B.5), ter um sentido próprio, pois aquele pode ser identificada como $\mathbb{E}[\mathbb{P}(X)]$. De maneira alternativa, ao se realizar uma substituição de variável, tal como $u_{i}=\mathbb{P}\left(x_{i}\right)$, o argumento é a média da variável transformada, enquanto a função probabilidade é a transformação. A entropia quadrática de Rényi é particularmente interessante por ser facilmente estimada a partir de dados amostrais.

A próxima Seção dá um vislumbre sobre como estimar a entropia.

\section{B.2 ESTIMANDO A ENTROPIA}

Do exposto na Seção anterior, surge o problema de como se estimar essas medidas. Embora haja diversas formas $[85,86]$, não há, na prática, muita clareza quanto ao comportamento das estimativas. Isso é especialmente verdade em termos do número de amostras temporais necessárias (duração do trecho observado da dinâmica). Note-se a quantidade de omissão quanto a aspectos práticos em [25]. 
De um modo geral, o problema comum para a estimativa dessas diversas formas de entropias reisde no fato da necessidade de um número muito grande de observações para convergência, mesmo em circunstâncias favoráveis (e pouco práticas) na ausência de ruído aditivo.

Uma proposta voltada para superar essas dificuldades foi introduzida por Parzen [54,87,88], através do chamado Método de Estimação de Densidade por Janelas (Kerneis) de Parzen (kernel density estimation - KDE). A proposta de Parzen tem sido largamente empregada em problemas de estimação de densidade de maneira não-paramétrica [89,90] e em estimação de taxa de disparo de neurônios [91]. A técnica do KDE se propõe a produzir um estimador consistente e uma quantia sensível a ruídos, baseada em séries empíricas de curta duração e, por isso, é usada nos métodos de kernel [16, 92].

Na Seção a seguir, exploram-se algumas importantes propriedades do KDE, introduzido anteriormente.

\section{B.2.1 Propriedades do estimador não-paramétrico de densidade por kernel}

Para o caso de uma única amostra, formalmente, tem-se a

Definição 5. Dada uma variável aleatória (v.a.) X, tal que suas amostras tenham a característica $\{\mathbf{x}(n)\}_{n=1}^{N} \sim$ i.i.d. $\left(\mu, \sigma^{2}\right)$, com uma pdf $f_{X}(x)$ desconhecida, essa pode ser estimada da seguinte maneira

$$
f_{X}(x)=\frac{1}{N \sigma} \sum_{n=1}^{N} \kappa\left(\frac{x-x(n)}{\sigma}\right),
$$

em que $\kappa(\cdot)$ é um kernel (radial) de Mercer, a ser escolhido, $\sigma$ é a banda de passagem/parâmetro de alisamento e $N$ é o número de amostras.

e, para sua versão vetorial, tem-se a

Definição 6. Dado um vetor aleatório $\mathbf{X}=\left\{\mathbf{x}_{i}(n)\right\}_{i=1}^{D}{ }_{n=1}^{N}$, tal que suas amostras tenham a característica $\{\mathbf{x}(n)\}_{i=1}^{D} \underset{n=1}{N} \sim$ i.i.d. ${ }_{D}(\boldsymbol{\mu}, \mathbf{\Sigma})$, com uma $p d f \mathbf{f}_{\mathbf{X}}(\mathbf{x})$ desconhecida, essa pode ser estimada da seguinte maneira

$$
\mathbf{f}_{\mathbf{X}}(\mathbf{x})=\frac{1}{N \sigma^{D}} \sum_{n=1}^{N} \mathbf{K}\left(\frac{\mathbf{x}-\mathbf{x}_{i}(n)}{\sigma}\right),
$$

em que $\mathbf{K}(\cdot)$ é uma matriz de kernel (radial) de Mercer, a ser escolhida e, novamente, $\sigma$ é a banda de passagem/parâmetro de alisamento e $N$ é o número de amostras.

Um ponto positivo para os estimadores acima é que se mostram estatisticamente consistentes, como Parzen prova em [87,90], ou seja,

$$
\begin{aligned}
\lim _{N \rightarrow \infty} \mathbb{E}\left[\widehat{f_{X}}(x)\right] & =f_{X}(x), \quad \lim _{N \rightarrow \infty} \mathbb{E}\left[\widehat{\mathbf{f}_{\mathbf{X}}}(\mathbf{x})\right]=\mathbf{f}_{\mathbf{X}}(\mathbf{x}), \\
\lim _{N \rightarrow \infty} \operatorname{Var}\left[\widehat{f_{X}}(x)\right] & =0, \quad \lim _{N \rightarrow \infty} \operatorname{Var}\left[\widehat{\mathbf{f}_{\mathbf{X}}}(\mathbf{x})\right]=\mathbf{0},
\end{aligned}
$$


fazendo com que o estimador da entropia quadrática de Rényi também seja.

Alguns métodos para escolha de parâmetro $\sigma$ foram propostos na literatura [16,90]. Silverman [90] criou uma regra (comumente chamada de "Regra de Silverman") quando o kernel Gaussiano é escolhido. Assim a banda de passagem, $\sigma$, para o caso univariado, ou seja, quando se trata de apenas um conjunto de dados é

$$
\sigma=0,9 A N^{-1 / 5}
$$

com

$$
A=\min \left\{s, \frac{q_{3}-q_{1}}{\Phi^{-1}(0,75)-\Phi^{-1}(0,25)}\right\},
$$

em que se o conjunto de dados é dado por $\{\mathbf{x}(n)\}_{n=1}^{N}$, então $s$ é o desvio padrão amostral, a diferença $\left(q_{3}-q_{1}\right)$ é a distância interquartílica amostral e a função $\Phi^{-1}(\cdot)$ é o quantil da normal padrão.

Entretanto, para o caso vetorial (multivariado), em que $\mathbf{X}=\left\{\mathbf{x}_{i}(n)\right\}_{i=1}^{D} \underset{n=1}{N}$, a banda de passagem, $\sigma$, em (B.10) é modificada para [90],

$$
\sigma=\operatorname{tr}\left(\mathbf{S}_{\mathbf{X}}\right)\left(\frac{4}{D+2}\right)^{1 /(D+4)} N^{-1 /(D+4)},
$$

em que $\mathbf{S}_{\mathbf{X}}$ é a matriz de covariância amostral de $\mathbf{X}$.

Há outras possibilidades de uso de kerneis, que podem ser apreciados na lista colocada na Seção 1.1 .7 e em [16,90]. 


\section{APÊNDICE C - SÉRIES DE VOLTERRA}

Para caracterizar um sistema linear, recorre-se à sua resposta impulsiva. Quando se trata de sistemas não-lineares, não existe uma forma canônica de representação assim tão simples. Em 1887, o matemático italiano Vito Volterra extendeu o conceito de convolução para o caso não-linear, por uma série de operadores integro-polinomiais com não-linearidade crescente. É possível dizer que esse conceito é uma generalização da série de Taylor $[12,93,94]$ (mapeamento entre dois espaços de funções, através de uma expansão em séries de potências).

\section{C.1 TEMPO CONTÍNUO}

A representação em série de Volterra de um sistema não-linear é feita conforme o diagrama de blocos da Figura 28, sendo sua formalização matemática dada por,

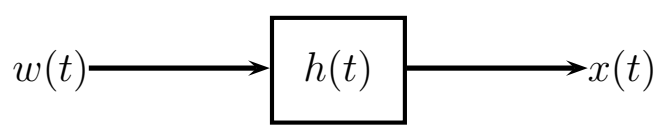

Figura 28 - Um típico processo de filtragem.

$$
x(t)=h^{(0)}(t)+\sum_{n=1}^{\infty} \int_{\mathbb{R}} h^{(n)}\left(t, \tau_{1}, \cdots, \tau_{n}\right) w\left(t-\tau_{1}\right) \cdots w\left(t-\tau_{n}\right) \mathrm{d} \tau_{1} \cdots \mathrm{d} \tau_{n},
$$

em que cada função $h^{(n)}(\cdot)$ é denominada kernel (do inglês, kernel) de Volterra de ordem $n$. Note que, $H_{0}[w(t)]=h^{(0)}(t)$ nada mais é do que o operador convolução, de sorte que para $n=1,2, \cdots$,

$$
H_{n}[w(t)]=\int_{\mathbb{R}} h^{(n)}\left(t, \tau_{1}, \cdots, \tau_{n}\right) w\left(t-\tau_{1}\right) \cdots w\left(t-\tau_{n}\right) \mathrm{d} \tau_{1} \cdots \mathrm{d} \tau_{n},
$$

ou seja, houve a generalização do operador convolução.

Rugh [94] batiza sistemas dinâmicos reproduzidos por (C.1) de sistemas de Volterra.

Os kernels de Volterra obedecem algumas propriedades:

\section{Causalidade dos kernels}

$$
h^{(n)}\left(t, \tau_{1}, \cdots, \tau_{n}\right)=0 \text {, para todo } \tau_{j}<0, j=1, \cdots, n \text {. }
$$

\section{Estabilidade dos kernels}


Pode-se mostrar que um sistema de Volterra é estável se e somente se seus kernels forem absolutamente somáveis, ou seja,

$$
\left\|h^{(n)}\left(t, \tau_{1}, \cdots, \tau_{n}\right)\right\|_{1}<\infty \text {, para todo } t \text { em } \mathbb{R}
$$

\section{Simetria dos kernels}

Como através de trocas de posições dos termos $x\left(t-\tau_{i}\right)$, na Equação C.1, podem levar a diferentes kernels de Volterra, representando sistemas de Volterra equivalentes, adota-se, sem perda de generalidade, a forma triangular de representá-los. Outras formas podem ser apreciadas em [94]. Assim, diz-se que um kernel $h^{(n)}\left(t, \tau_{1}, \cdots, \tau_{n}\right)$ está na forma triangular, quando,

$$
\tau_{i+j}<\tau_{j} \Rightarrow h^{(n)}\left(t, \tau_{1}, \cdots, \tau_{j}, \cdots, \tau_{i+j}, \cdots, \tau_{n}\right)=0,
$$

para todo $i, j$ em $\{\cdots,-1,1, \cdots\}$, tais que $i+j \leq n$.

\section{Multilinearidade do operador de Volterra}

$$
H_{n}[c w(t)]=c^{n} H_{n}[w(t)]
$$

em que $c$ é um número real e constante.

Já, em relação à variabilidade do sinal de entrada do sistema, sabe-se que é possível classificá-lo como: (A) fracamente estacionário ou (B) não-estacionário. Em (A), é necessário que sua média seja constante e sua função de autocorrelação dependa apenas da diferença entre instantes de tempos. Logo, quando se trata do caso (A), a dependência da variável do tempo $t$, que ocorre em (C.1) e (C.2) pode ser omitida.

Portanto, em vista das condições acima e de (C.1), tem-se que um sistema nãolinear variante no tempo, causal, é escrito como,

$$
x(t)=h^{(0)}(t)+\sum_{n=1}^{\infty} \int_{0}^{\infty} \int_{0}^{\tau_{n}} \cdots \int_{0}^{\tau_{1}} h^{(n)}\left(t, \tau_{1}, \cdots, \tau_{n}\right) \prod_{i=1}^{n} w\left(t-\tau_{n}\right) \mathrm{d} \tau_{1} \cdots \mathrm{d} \tau_{n},
$$

enquanto a versão invariante no tempo,

$$
x(t)=h^{(0)}+\sum_{n=1}^{\infty} \int_{0}^{\infty} \int_{0}^{\tau_{n}} \cdots \int_{0}^{\tau_{1}} h^{(n)}\left(\tau_{1}, \cdots, \tau_{n}\right) \prod_{i=1}^{n} w\left(t-\tau_{n}\right) \mathrm{d} \tau_{1} \cdots \mathrm{d} \tau_{n},
$$

sempre admitindo que os kernels de Volterra estão na forma triangular.

\section{C.2 TEMPO DISCRETO (CASO ESTACIONÁRIO)}

De (C.8), Alper, em [73,95], introduziu a série de Volterra discreta:

$$
x(n)=h^{(0)}+\sum_{r=1}^{p} \sum_{k_{1}=0}^{N_{r}} \sum_{k_{2}=k_{1}}^{N_{r}} \ldots \sum_{k_{r}=k_{r-1}}^{N_{r}} h^{(r)}\left(k_{1}, \cdots, k_{r}\right) \prod_{i=1}^{r} w\left(n-k_{i}\right),
$$


em que $N_{r}$ representa a memória do kernel $h^{(r)}(\cdot)$, em amostras. Um modelo na forma (C.9) recebe o nome de sistema de tempo discreto de Volterra ou filtro de Volterra.

Sob a hipótese de triangularidade dos kernels, fazendo $m=\max _{1 \leq r \leq p}\left\{N_{r}\right\}$, observa-se que o número de coeficientes de uma estrutura expressa pela Equação C.9 é dada por [94],

$$
N_{p, m}=1+\sum_{r=1}^{p}\left(\begin{array}{c}
m+r-1 \\
r
\end{array}\right) \text {. }
$$

Como é afirmado em [94], (C.10) despreza combinações de coeficientes $\tau_{i}$ que não satisfaçam (C.5). Uma das dificuldades de aplicação dos Filtros de Volterra se deve ao crescimento quase exponencial de $N_{p, m}$.

\section{C.2.1 Sobre a convergência}

Pela sua própria característica enquanto expansão em série de potência, a convergência da série de Volterra com infinitos termos não é garantida para sinais de entrada arbitrários, requerendo restrições para que isso aconteça [94], ou seja,

1. intervalo de tempo (sinal de entrada com suporte finito);

2. sinal de entrada deve ser limitado, para produzir saída com valores limitados;

condições cujo controle não são simples, conforme discutem [94, 96, 97]. 


\section{APÊNDICE D - MODELOS ORIENTADOS À BLOCOS}

Como visto no Apêndice C, a série de Volterra, por ser de natureza não-paramétrica, leva a modelos com um número muito elevado de coeficientes, mesmo para sistemas de ordem reduzida, como descreve [98,99]. A fim de se diminuir essa alta complexidade dos Sistemas de Volterra, foram propostos os Modelos Orientados à Blocos (MOB) [12], que são estruturas compostas pela interconexão, em cascata ou paralelo, de sistemas lineares contendo não-linearidades estáticas (sem memória) ${ }^{1}$.

No restante deste Apêndice, os modelos MOB mais comuns são apresentados [12] enquanto outros podem ser apreciados com maiores detalhes em [100].

\section{D.1 MODELO DE HAMMERSTEIN}

O modelo de Hammerstein, é construído por um bloco de não-linearidade estática $g(\cdot)$, em cascata com um sistema linear, representado pela resposta impulsiva $h(n)$ [101], como se observa no típico diagrama de blocos da Figura 29.

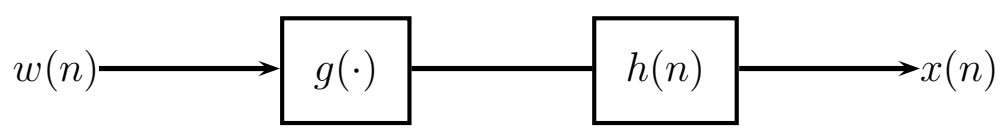

Figura 29 - Típico MOB de Hammerstein.

Assim, da Figura 29 é possível escrever,

$$
x(n)=\sum_{k=0}^{\infty} h(k) g[w(n-k)] .
$$

Expressando-se $g(x)$ através de combinação linear de funções de base $g_{i}(x)$ [101], tem-se,

$$
x(n)=\sum_{k=0}^{\infty} h(k) \sum_{r=0}^{p} \alpha_{r} g_{r}[w(n-k)]=\sum_{r=0}^{p} \alpha_{r} \sum_{k=0}^{\infty} h(k) g_{r}[w(n-k)],
$$

em que $\left\{\alpha_{r}\right\}_{r=0}^{p}$ são os coeficientes da série. É comum fazer-se,

$$
g_{r}(x)=x^{r},
$$

ou seja, utilizar uma expansão polinomial da não-linearidade.

\section{D.2 MODELO DE WIENER}

Agora, colocando-se a não-linearidade estática $g(\cdot)$ em cascata, após, o sistema linear $h(n)$, obtém-se outro MOB, denominado de modelo de Wiener, bastante utilizado [101-103]. O diagrama de blocos mais comum é dado na Figura 30.

1 Não-linearidade estática ou sem memória é aquela em que o efeito da não-linearidade seja instantâneo, no sinal de entrada. 


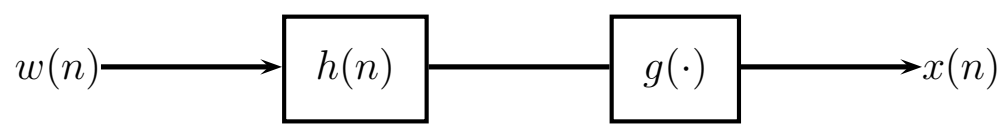

Figura 30 - Típico MOB de Wiener.

Como foi apresentado na Seção D.1, utilizando-se a mesma forma de expansão da não-linearidade, o modelo polinomial de Wiener fica escrito como,

$$
x(n)=g\left[\sum_{k=0}^{\infty} h(k) w(n-k)\right]=\sum_{r=0}^{p} \alpha_{r} g_{r}\left[\sum_{k=0}^{\infty} h(k) w(n-k)\right] .
$$

\section{D.3 MODELO DE WIENER-HAMMERSTEIN}

Uma alternativa aos MOB das Seções D.1 e D.2 é a combinação das duas técnicas anteriores, a que se dá o nome de modelo de Wiener-Hammerstein [104], para que se tenha a possibilidade de modelar comportamentos mais complexos. O diagrama de blocos da Figura 31 apresenta um possível modelo.

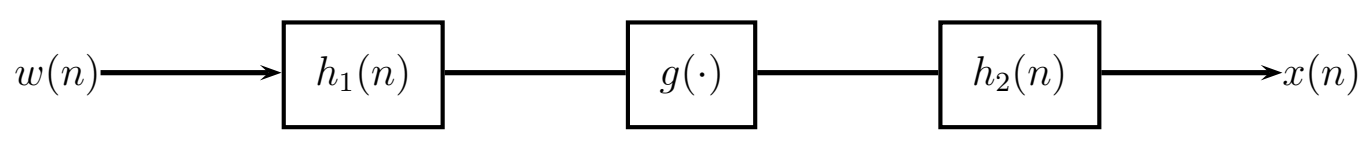

Figura 31 - Possível MOB de Wiener-Hammerstein.

Note que a não-linearidade estática é colocada entre sistemas lineares. Dessa forma, o modelo de Wiener-Hammerstein será,

$$
\begin{aligned}
x(n) & =\sum_{k=0}^{\infty} h_{2}(k) g\left[\sum_{m=0}^{\infty} h_{1}(l) w(n-k-m)\right] \\
& =\sum_{r=0}^{p} \alpha_{r} \sum_{k=0}^{\infty} h_{2}(k) g_{r}\left[\sum_{m=0}^{\infty} h_{1}(l) w(n-k-m)\right] .
\end{aligned}
$$




\section{APÊNDICE E - MODELOS NARMAX}

No Apêndice C foi visto que o sistema de Volterra é uma generalização da integral de convolução e, também, que há uma dificuldade prática [101] na identificação de sistemas, ao se utilizar séries de Volterra, pois, mesmo para ordens baixas, o número de coeficientes dos núcleos, a se determinar, é muito alto. Isso se deve pelo fato da série de Volterra tentar explicar a saída $\{x(t)\}$ somente em função da entrada $\{w(t)\}$. Na tentativa de se reduzir o número de parâmetros, utilizam-se funções de base ortonormais [105].

\section{E.1 MODELOS NÃO-LINEARES DE REGRESSÃO ESTOCÁSTICOS}

A motivação para a representação de sistemas não-lineares via modelos NARMAX, que compactam ainda mais o número de parâmetros, uma vez que a saída (em um dado instante) relaciona-se com a entrada, em instantes anteriores, podendo, também, incluir termos adicionais para o modelamento de perturbações $[101,106,107]$ ao invocar uma relação de recorrência, em tempo discreto. Sua forma matemática é dada por:

$$
\begin{aligned}
& x(n)=f[ x(n-1), \cdots, x\left(n-k_{x}\right), \cdots, w(n-1), \cdots, w\left(n-k_{w}\right), \cdots \\
&\left.\cdots, e(n), \cdots, e\left(n-k_{e}\right)\right],
\end{aligned}
$$

em que $f(\cdot)$ é uma função-linear, $k_{x}, k_{w}$ e $k_{e}$ são os maiores atrasos em $x(n)$ (saída), $w(n)$ (entrada) e $e(n)$ (variável exógena). Assim, o nome dado a essa classe de processos é NãoLinear Autorregressivo de Média Móvel com Entrada Exógena (Nonlinear Autoregressive Moving Average with Exogenous Inputs - NARMAX).

Um exemplo para a função $f(\cdot)$, na representação NARMAX (sem atraso puro de tempo), comumente usada é um polinômio, como mostra (E.2) [101],

$$
x(n)=\sum_{i} c_{i} \prod_{j=1}^{k_{x}} x(k-j) \prod_{r=1}^{k_{w}} w(k-r) \prod_{q=1}^{k_{e}} e(k-q) .
$$

O modelo (E.2) é um exemplo de regressão estocástica não-linear que evita viés estatístico nas estimativas dos parâmetros [101]. Porém, em muitas situações considera-se apenas a parte autorregressiva (AR) do modelo, pois como afirma [108], modelos AR têm a capacidade de capturar efeitos de dependência temporal entre o histórico de varáveis, dando lugar aos modelos NARX - Nonlinear Autoregressive with Exogenous Inputs,

$$
x(n)=f\left[x(n-1), \cdots, x\left(n-k_{x}\right), \cdots, w(n-1), \cdots, w\left(n-k_{w}\right)\right] .
$$

Em [107], é observado que (E.3) é bastante geral para representar um sistema de tempo discreto não-linear. De maneira simples, é comum definir a função $f(\cdot)$ através de 
polinômios (a exemplo do que foi feito para o modelo NARMAX acima) e, portanto, (E.3) fica [101],

$$
x(n)=\sum_{r=1}^{p} \sum_{p=0}^{m} \sum_{k_{1}, k_{m}}^{k_{x}, k_{w}} c_{p, m-p}\left(k_{1}, \cdots, k_{m}\right) \prod_{i=1}^{l} w\left(n-k_{i}\right) \prod_{i=l+1}^{m} x\left(n-k_{i}\right),
$$

em que

$$
\sum_{k_{1}, k_{m}}^{k_{x}, k_{w}} \equiv \sum_{k_{1}=1}^{k_{x}} \cdots \sum_{k_{m}=1}^{k_{w}}
$$

É factível que se utilizem outras escolhas para $f(\cdot)$, mas não é comum. Em [100, 101] mostra-se o uso de funções racionais e se discute alguns aspectos relativos à sua identificação.

\section{E.1.1 Relação dos modelos NARMAX/NARX com as séries de Volterra}

Acima, discutiu-se uma motivação para se obter equações de diferenças para o ajuste de mapas não-lineares. Para tanto, foi exposto que as séries de Volterra, por serem uma generalização da integral de convolução, ou seja, uma abordagem não paramétrica, utilizam um número muito grande de coeficientes mesmo para mapas de baixa ordem.

Como se sabe, uma equação de diferenças é uma representação paramétrica de um sistema e, portanto, mais compacta do que a resposta impulsiva. No caso de mapas mais gerais, a resposta impulsiva possui infinitos termos (teoricamente) e uma representação exata é muito difícil. Assim, usa-se como aproximação a resposta impulsiva com duração finita, que ainda sim possui muitos coeficientes.

Além das características acima, os métodos de identificação de modelos NARMAX/ NARX não possuem estabilidade estatística aceitável, como os que são reportados em [109]. 


\section{ANEXO A - LISTA DE PUBLICAÇÕES}

A1. MASSAROPPE, L.; CADUDA, F.; BACCALÁ, L. A. On the performance of waveletbased long-memory parameter estimation. In: ASSOCIAÇ $\widetilde{O}$ BRASILEIRA DE ESTATÍSTICA (ABE). 21o. SINAPE - Simpósio Nacional de Probabilidade e Estatística. Natal, RN: ABE, 2014.

A2. MASSAROPPE, L.; BACCALÁ, L. A. Detecting nonlinear Granger causality via the kernelization of partial directed coherence. In: Proceedings of the 60th World Statistics Congress of the International Statistical Institute, ISI2015. Rio de Janeiro, RJ: International Statistical Institute, The Hague, The Netherlands, 2015. p. 20362041.

A3. MASSAROPPE, L.; BACCALÁ, L. A. A simulation comparison between fractional differencing exponent estimators. In: ASSOCIAÇÃO BRASILEIRA DE ESTATÍSTICA (ABE). XVI Escola de Séries Temporais e Econometria. Campos do Jordão, SP: ABE, 2015.

A4. MASSAROPPE, L.; BACCALÁ, L. A. Kernel-nonlinear-PDC extends Partial Directed Coherence to detecting nonlinear causal coupling. In: 2015 37th Annual International Conference of the IEEE Engineering in Medicine and Biology Society (EMBC). Milan: [s.n.], 2015. p. 2864-2867.

A5. MASSAROPPE, L.; BACCALÁ, L. A. Causal connectivity via kernel methods: Advances and challenges. In: 2016 38th Annual International Conference of the IEEE Engineering in Medicine and Biology Society (EMBC). Florida: [s.n.], 2016. 
A.1 SINAPE 2014

\title{
On the Performance of Wavelet-Based Long-Memory Parameter Estimation
}

\author{
Lucas Massaroppe, Flavio Caduda and Luiz A. Baccalá \\ Escola Politécnica - Universidade de São Paulo \\ lucasmassaroppe@usp.br, caduda@usp.br, baccala@lcs.poli.usp.br
}

\begin{abstract}
After reviewing wavelet techniques used to estimate the long-memory parameter $d$ used in ARFIMA models, Monte Carlo simulations are used to evaluate the performance of different discrete wavelet transforms under various mother wavelet choices. By comparing computed small sample bias, standard deviations and mean-square errors from the different methods, MODWPT's (Maximum Overlap Discrete Wavelet Packet Transform) is shown to outperform all other options under a minimum mean-square error criterion using the $\mathbf{D}(4)$ wavelet filter.
\end{abstract}

Keywords: Fractionally integrated models, Long-memory, Wavelets

\section{Introduction}

Interest in long-memory (LM) processes has grown rapidly due to realization of their applicability to internet traffic and hydrological time series data $[7,11]$. The correlation for observations far apart in time for long-memory data is non-negligible because its autocorrelation function has nonexponential decay $[2,5]$. An LM model of particular interest is the Autoregressive Moving Average Fractionally Integrated Models, denoted as $\operatorname{ARFIMA}(p, d, q)$, where $d$ is the LM paramater, while $p$ and $q$ are the usual ARMA order parameters. It can be shown that for i.i.d. Gaussian innovations and $|d|<1 / 2$, the resulting ARFIMA $(0, d, 0)$ process is stationary $[1,2]$. The objective here is to compare estimates of the parameter $d$ with wavelet-based least-squares methods, as proposed by Jensen [9] and Whitcher $[12,13]$ using Monte Carlo simulations.

The rest of this paper is organized as follows: Section 2 provides a brief review long-memory processes, whose wavelet domain estimation of long-memory parameter is summarized in Section 3. A short discussion follows (Section 4) and the ensuing results (Section 5). Conclusions are collected in Section 6. 


\section{Fractionally integrated processes}

$\operatorname{An} \operatorname{ARFima}(0, d, 0)\{x(n)\}_{n \in \mathbb{Z}}$ is defined by,

$$
(1-L)^{d} x(n)=w(n),
$$

where $\{w(n)\}_{n \in \mathbb{Z}} \sim$ i.i.d. $\mathbf{N}\left(0, \sigma_{w}^{2}\right),|d|<1 / 2$ and $(1-L)^{d}$ constitutes the fractional differencing operator, whose binomial expansion is

$$
(1-L)^{d}=\sum_{j=0}^{\infty}\left(\begin{array}{l}
d \\
j
\end{array}\right)(-L)^{j}=\sum_{j=0}^{\infty} \frac{\Gamma(j-d)}{\Gamma(j+1) \Gamma(-d)} L^{j}
$$

where $L$ denotes the unit delay operator and $\Gamma(\cdot)$, the gamma function. Note that the process described by the equation $(1-L) x(n)=x(n)-x(n-1)$ is integrated of order one (denoted as $I(1))$. Therefore, Equation 1 is a generalization of integrated processes.

\section{Estimating the long-memory parameter}

There are several methods for estimating $d$. Here we concentrate on methods that employ wavelets, namely (a) the Discrete Wavelet Transform (DWT), (b) Maximal Overlap DWT (MODWT) and their wavelet packet generalizations: (c) DWPT and (d) MODWPT (as discussed in [12]). The interested reader can find a detailed description of the DWT-based estimators in $[3,9,10]$ and the MODWT-based transform in $[3,10,13]$. The packet transforms versions DWPT and MODWPT-based can be found in $[3,6,12]$

Using wavelets to decompose the signal in its respective time-scale subbands, Jensen [9] showed the existence of a log-linear relationship between wavelet coefficient variances and its scale, where the slope of the fitted line is proportional to $d$. Therefore, Jensen proposed a method to compute it, referred here as Ordinary Wavelet Least Squares - OWLS method, which he proves to be a consistent estimator for $d[9]$.

All such least squares $d$ estimators are consistent and asymptotically normal as shown by Jensen [9] and Whitcher and Jensen [13]. Furthermore, OWLS is computationally appealing [9,11].

\section{Simulations}

OWLS approach robustness for estimating $d$ was probed by simulating processes for $d$ in $\{0.1,0.2,0.3,0.4\}$ and realization lengths $N$ in $\{128,256,512,1024,2048\}$. 10000 Monte Carlo simulations were conducted for each parameter/length combination, where the first 10000 points of each series were discarded to avoid transient phenomena. Long-memory process simulation followed the guidelines in [8], with additional enhancements. These results are tabulated and available for download at: 
http://www.lcs.poli.usp.br/ baccala/longmemorywavepaper.zip.

The estimates for $d$ were guaranteed to have good statistical properties by following the recommendations contained in $[3,4,9,10,13]$. In the simulations, two different wavelet filter types were considered: the Extremal Phase (Haar and $\mathbf{D}(4))$ and Least Asymmetric $(\mathbf{L A}(8))$ filters. This illustrated the statistical performance and allowed suggesting the best wavelet filter to use.

\section{Results}

The estimated mean, bias, standard deviation (SD) and mean-square error MSE for $\widehat{d}$ were computed under the various OWLS estimation options. The simulations revealed interesting results concerning finite-sample bias. The OWLS method tends to underestime $d$, i.e., its bias is almost always negative. As $d$ increases and $N$ remains fixed, its bias decreases. For each $d$ value, the absolute bias almost always decreases as $N$ grows, confirming consistency. As for $\mathrm{SD}$, we see that at each $d$, as $N$ increases, its value decreases (Figure 1).

Analogously, the MSE of the OWLS method showed a similar pattern for all four types of wavelet transforms and filters. At each $d$, the MSE declined as $N$ grew. For fixed $N$ the OWLS method was insensitive to changes in value of $d$ despite the existence of a hierarchy of performance according to wavelet transform type and filter (Figure 2(a)).

Figure 2(b) shows the boxplots of OWLS method, when using $N=512$ data points. Note that the circle inside the boxes marks the mean value of the distribution. Theses boxplots show that OWLS used along with the MODWPT and the $\mathbf{D}(4)$ filter gives the best estimation performance for $d$ leading to the lowest variance.

\section{Conclusion}

This work briefly overviewed consistent fractional differencing parameter estimation under OWLS. Through Monte Carlo simulation (Section 5) it was possible to investigate and confirm the consistency properties for $d[9,12,13]$ whose best performance results were achieved when the OWLS method was implemented with the MODWPT for the $\mathbf{D}(4)$ wavelet filter whereby $d$ had the smallest mean-squared error.

\section{Acknowledgments}

CNPq Grant 307163/2013-0 to L.A.B. is also gratefully acknowledged. L.M. gratefully acknowledges support from an institutional Ph. D. CAPES Grant. 

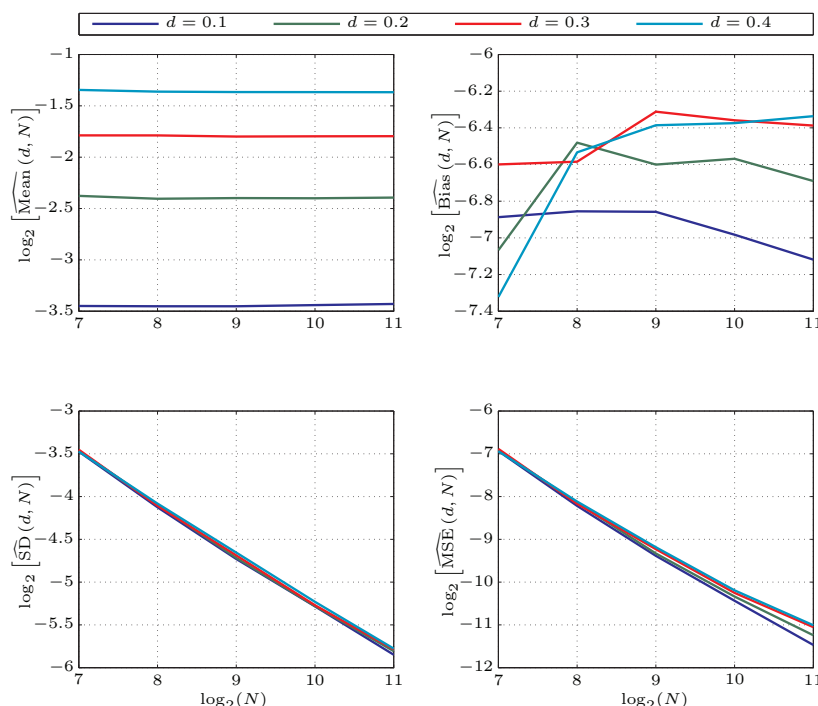

Figure 1: Beginning from top left panel, in clockwise direction, $\log _{2}-\log _{2}$ plots of estimates of the mean, bias, standard-deviation (SD) and mean-square error (MSE), in function of the segment length, $N$, for the OWLS used with the MODWPT, along with the $\mathbf{D}(4)$ filter. 


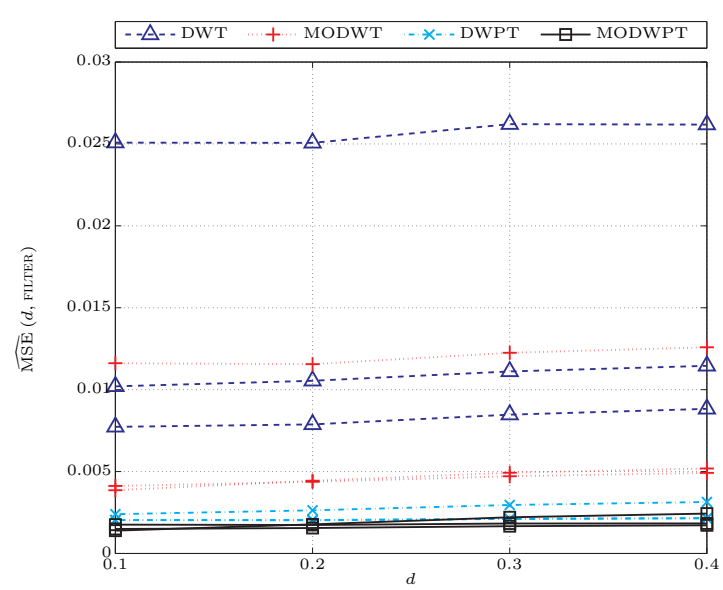

(a) Mean-square error as function of $d$, with $N=512$, knowing that: $\widehat{\operatorname{MSE}}(d, \mathbf{D}(4))<\widehat{\operatorname{MSE}}(d, \mathbf{L A}(8))<\widehat{\operatorname{MSE}}(d$, Haar $)$, for the DWT, MODWT,

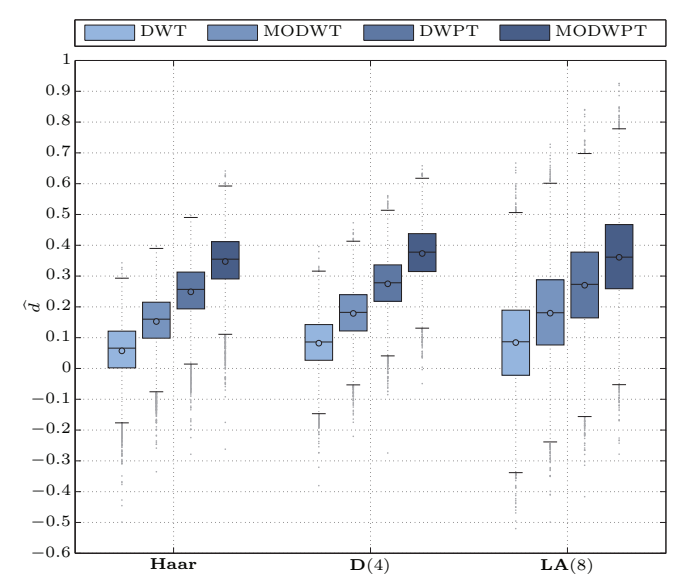

(b) OWLS method with $N=512$ data points. Comparison between different filters, Haar, D(4), LA (8), used to estimate $d$ for each wavelet transorm (DWT, MODWT, DWPT, MODWPT). Lower variance is noticed for $\mathbf{D}(4)$ using MODWPT

Figure 2: Comparison between different settings of OWLS, with $N=512$ 


\section{References}

[1] Jan Beran. Statistics for long-memory processes. Monographs on statistics and applied probability, 61. Chapman \& Hall, October 1994.

[2] Peter J. Brockwell and Richard A. Davis. Time series: Theory and methods. Springer series in statistics. Springer-Verlag, New York, 2nd edition, 1991.

[3] Ramazan Gençay, Faruk Selçuk, and Brandon Whitcher. An introduction to wavelets and other filtering methods in finance and economics. Academic Press, San Diego, California, 2002.

[4] Tilmann Gneiting, Hana Ševčíková, and Donald B. Percival. Estimators of fractal dimension: Assessing the roughness of time series and spatial data. Statistical Science, 27(2):247-277, May 2012.

[5] Clive William John Granger and Roselyne Joyeux. An introduction to long-memory time series models and fractional differencing. Journal of Time Series Analysis, 1(1):15-29, January 1980.

[6] Dominique Guegan and Zhiping Lu. Wavelet method for locally stationary seasonal long memory processes. Documents de travail du Centre d'Economie de la Sorbonne 09015, Université PanthéonSorbonne (Paris 1), Centre d'Economie de la Sorbonne, March 2009.

[7] Harold Edwin Hurst. Long term storage capacity of reservoirs. Transactions of the American Society of Civil Engineers, 116:770-799, 1951.

[8] Andreas Noack Jensen and Morten Ørregaard Nielsen. A fast fractional difference algorithm. Working Papers 1307, Queen's University, Department of Economics, April 2013.

[9] Mark J. Jensen. Using wavelets to obtain a consistent ordinary least squares estimator of the long memory parameter. Journal of Forecasting, 18:17-32, 1999.

[10] Donald B. Percival and Andrew T. Walden. Wavelet methods for time series analysis. Cambridge Series in Statistical and Probabilistic Mathematics. Cambridge University Press, Cambridge, 2000.

[11] Darryl Veitch and Patrice Abry. A wavelet-based joint estimator of the parameters of long-range dependence. IEEE Transactions on Information Theory, 45(3):878-897, April 1999.

[12] Brandon Whitcher. Wavelet-based estimation for seasonal long-memory processes. Technometrics, 46(2):225-238, 2004 .

[13] Brandon Whitcher and Mark J. Jensen. Wavelet estimation of a local long memory parameter. Exploration Geophysics, 31(2):94-103, January 2000. 


\section{A.2 ISI 2015}

1512015

Detecting Nonlinear Granger Causality via the kernelization of Partial Directed Coherence

Lucas Massaroppe*

University of São Paulo, São Paulo, Brazil — lucasmassaroppe@usp.br

Luiz A. Baccalá

University of São Paulo, São Paulo, Brazil — baccala@lcs.poli.usp.br

\section{Abstract}

Though by now classic for inferring causal relations between time series, linear vector autoregressive models fail to capture certain types of nonlinear coupling. Here we explore a new concept kernel-nonlinearPartial Directed Coherence and its ability to overcome those limitations, via a simulated example of its asymptotic behaviour.

Keywords: Nonlinear-Granger causality; Partial Directed Coherence; Inference; Detection; Kernel Processing.

\section{Introduction}

Thanks to the well understood properties of linear vector autoregressive (VAR) models, convergence and asymptotics among them (Lütkepohl, 2005), they have long been the methods of choice for inferring Granger causality (Granger, 1969) even when nonlinear time series are under investigation (Schelter et al., 2006) despite their proven inability to portray polynomial couplings of even power (Massaroppe et al., 2011). Alternatives exist, both parametric (Faes et al., 2008; He et al., 2014; Pereda et al., 2005) and nonparametric (Hlaváčková-Schindler et al., 2007; Marinazzo et al., 2008; Paluš et al., 2001), they share the weakness of requiring many data points for proper convergence or, sometimes, require explicit prior knowledge of the coupling structure. Additional shortcomings stem from having to deal with many parameters and/or the minimization of nonconvex functionals.

Here we examine a new parametric approach, termed kernel-nonlinear-Partial Directed Coherence (Kannan and Tangirala, 2014) based on computing the partial directed coherence (PDC) (Baccalá and Sameshima, 2001) of properly 'kernelized' data. The present approach differs from Kannan and Tangirala (2014) in two important ways: (a) the use of polynomial kernels instead of the gaussian kernel adopted by the latter and (b) in the use of implicit kernel mapping generalizing Kumar and Jawahar (2007) to the multivariate time series case.

After a brief recap (Section 2), simulation of a simple model borrowed from Massaroppe et al. (2011) illustrates the potential viability (Section 3) of applying our recently developed asymptotic causality detection criteria (Baccalá et al., 2013; Takahashi et al., 2010) to this new form of PDC. We end with a brief discussion (Section 4).

\section{The Method}

To extend partial directed coherence (PDC) (Baccalá and Sameshima, 2001) to capture more general couplings, we propose to represent the observed data $x_{i}(n)(1 \leq i, n \leq K \ll N)$ through a Kernel Vector Autoregressive $(k V A R)$ model

$$
\phi[\mathbf{x}(n)]=\sum_{r=1}^{p} \mathbf{A}^{\phi}(r) \phi[\mathbf{x}(n-r)]+\boldsymbol{\vartheta}^{\phi}(n),\left\{\boldsymbol{\vartheta}^{\phi}(n)\right\}_{n \in \mathbb{Z}} \sim \text { i.i.d.WN }\left(\mathbf{0}, \boldsymbol{\Sigma}_{\boldsymbol{\vartheta}^{\phi}}\right),
$$

where $\phi(\cdot)$ stands for a mapping (Parzen, 1959) taking the data from its input space $\mathcal{X}$ to a suitably defined feature space $\mathbf{F}$, by choosing $\phi(\cdot)$ so that $\mathbb{E}\left\{\phi\left[x_{i}(n)\right] \phi\left[x_{i}(n-k)\right]\right\}=\mathbb{E}\left\{\kappa\left[x_{i}(n), x_{i}(n-k)\right]\right\}$ where $\kappa(\cdot)$ is a Mercer kernel.

This leads naturally to the definition of kernel-nonlinear-PDC as

$$
{ }_{\kappa \eta} \pi_{i j}(f)=\frac{\bar{A}_{i j}^{\phi}(f) / \sqrt{\sigma_{i i}^{\phi}}}{\sqrt{\overline{\mathbf{a}}_{j}^{\phi \mathrm{H}}(f) \boldsymbol{\Sigma}_{\vartheta^{\phi}}^{-1} \overline{\mathbf{a}}_{j}^{\phi}(f)}}
$$


where

$$
\bar{A}_{i j}^{\phi}(f)=\delta_{i j}-\sum_{l=1}^{p} a_{i j}^{\phi}(l) \mathrm{e}^{-\mathbf{i} 2 \pi f l},\left(\mathbf{i}^{2}=-1\right),
$$

with $a_{i j}^{\phi}(l)$ representing adequately fit kVAR model coefficients, while $\overline{\mathbf{a}}_{j}^{\phi}(f)$ represent the columns of the $\left[\bar{A}_{i j}^{\phi}(f)\right]$ matrix.

To fit (1) we extend the univariate model fitting approach from Kumar and Jawahar (2007) to the multivariate case by rewriting it as

$$
\mathbf{\Phi}_{i}=\mathbf{A}^{\phi}\left(\mathbf{X} \mathbf{J}_{i}\right)^{\top}+\boldsymbol{\Theta}_{i}^{\phi},
$$

where

$$
\begin{aligned}
& \boldsymbol{\Phi}_{i}=(\phi[\mathbf{x}(1)], \cdots, \phi[\mathbf{x}(N)]) \quad(K \times N), \quad \mathbf{A}^{\phi}=\left(\mathbf{A}^{\phi}(1), \cdots, \mathbf{A}^{\phi}(p)\right) \quad(K \times K p), \\
& \mathbf{X}(n)=\left[\begin{array}{c}
\phi[\mathbf{x}(n)] \\
\vdots \\
\phi[\mathbf{x}(n-p+1)]
\end{array}\right] \quad(K p \times 1), \quad \mathbf{J}_{i}=\left[\begin{array}{c}
\mathbf{0}_{(i-K p-1) \times K p} \\
\mathbf{I}_{K p} \\
\mathbf{0}_{(N-i+1) \times K p}
\end{array}\right],
\end{aligned}
$$

which upon the application of the vec-operator

$$
\boldsymbol{\varphi}_{i}=\operatorname{vec}\left(\boldsymbol{\Phi}_{i}\right) \quad(K N \times 1), \quad \boldsymbol{\alpha}^{\phi}=\operatorname{vec}\left(\mathbf{A}^{\phi}\right) \quad\left(K^{2} p \times 1\right), \quad \boldsymbol{\theta}_{i}^{\phi}=\operatorname{vec}\left(\boldsymbol{\Theta}_{i}^{\phi}\right) \quad(K N \times 1),
$$

leads to

whose least squares solution

$$
\boldsymbol{\varphi}_{i}=\left(\mathbf{X J}_{i} \otimes \mathbf{I}_{K}\right) \boldsymbol{\alpha}^{\phi}+\boldsymbol{\theta}_{i}^{\phi},
$$

$$
\widehat{\boldsymbol{\alpha}}^{\phi}=\left(\sum_{i=p+1}^{N} \mathbf{J}_{i}^{\top} \mathbf{X}^{\top} \mathbf{X} \mathbf{J}_{i}\right)^{-1}\left(\sum_{i=p+1}^{N} \mathbf{J}_{i}^{\top} \mathbf{X}^{\top} \boldsymbol{\varphi}_{i}\right)=\left(\sum_{i=p+1}^{N} \mathbf{J}_{i}^{\top} \mathbf{K} \mathbf{J}_{i}\right)^{-1}\left(\sum_{i=p+1}^{N} \mathbf{J}_{i}^{\top} \mathbf{K}^{i}\right),
$$

where $\mathbf{K}=\mathbf{X}^{\top} \mathbf{X}$ is the block-Kernel matrix and the matrix $\mathbf{X}^{\top} \boldsymbol{\varphi}_{i}$ equals $\mathbf{K}$ 's $i$-th column $-\mathbf{K}^{i}$. In computing $\mathbf{K}$, the usual kernel centering procedure (Kumar and Jawahar, 2007; Shawe-Taylor and Cristianini, 2004) was adopted together with proper ridge regression stabilization procedures, with $\lambda=$ $10^{-6}$. Model orders were estimated using a suitably adapted Hannan-Quinn criterion.

Note that $(4,7)$ generalize the univariate time series formulations of Kallas et al. (2013); Kumar and Jawahar (2007), while (2) employs the information theoretical formulation of PDC (Takahashi et al., 2010), which more closely portrays the notion of information flow between series (Baccalá and Sameshima, 2014a,b).

\section{Numerical Illustration}

To gauge approach performance we simulated the system

$$
\left[\begin{array}{l}
x_{1}(n) \\
x_{2}(n)
\end{array}\right]=\left[\begin{array}{cc}
2 R \cos \left(2 \pi f_{0}\right) & 0 \\
0 & \xi
\end{array}\right]\left[\begin{array}{l}
x_{1}(n-1) \\
x_{2}(n-1)
\end{array}\right]+\left[\begin{array}{cc}
-R^{2} & 0 \\
0 & 0
\end{array}\right]\left[\begin{array}{l}
x_{1}(n-2) \\
x_{2}(n-2)
\end{array}\right]+\left[\begin{array}{ll}
0 & 0 \\
c & 0
\end{array}\right]\left[\begin{array}{l}
x_{1}^{2}(n-1) \\
x_{2}^{2}(n-1)
\end{array}\right]+\left[\begin{array}{l}
w_{1}(n) \\
w_{2}(n)
\end{array}\right],
$$

with $R=0.99$ chosen to produce a sharp resonance at $f_{0}=0.1$ and for $\xi=-0.9$, where $w_{i}(n)$ are independent zero mean and unit variance innovations. The quadratic coupling constant $c$ was in turn allowed to take different values $(\{0.10,0.50,1.00\})$ and realization lengths $N$ in $\{100,250,500,750,1000\}$ taken after sufficiently long burn-in times as in Massaroppe et al. (2011), which also shows a sample run of this example and its allied result of linear PDC's inability to capture coupling without prior kernelization.

Figure 1 shows the result of applying the detection criteria described in Baccalá et al. (2013) to the computed $k n P D C$ of a typical realization under a bi-quadratic kernel, showing that it is possible detect the effect $\phi\left[x_{1}(n)\right] \rightarrow \phi\left[x_{2}(n)\right]$ at $f=f_{0}=0.1$. Threshold computations were made based on the covariance structure from the data before kernelization.

To investigate Baccalá et al. (2013)'s detection capabilities we performed a Monte Carlo simulation comprising 10,000 repetitions whose distribution behaviour is exemplified in Figure 3 for $c=1.00$ and $N=750$. More details for other parameters are shown on Table 1 for both quadratic and bi-quadratic kernels. 

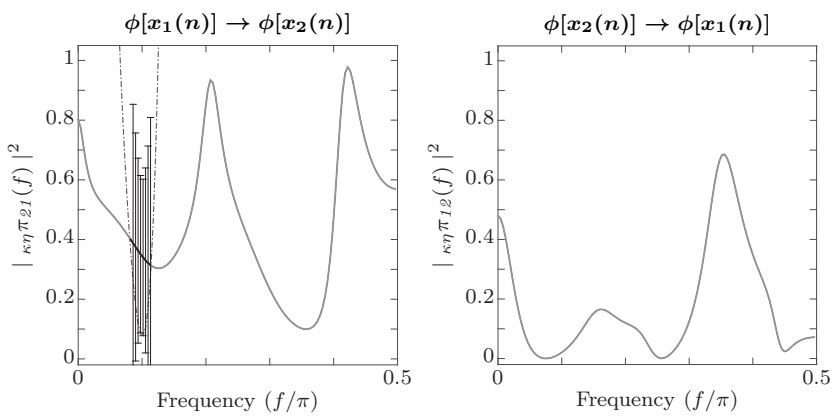

Figure 1: $\mathrm{knPDC}$ for a sample run of (9) under $\kappa(\mathbf{x}, \mathbf{y})=\left(\mathbf{x}^{\top} \mathbf{y}\right)^{4}$ capable of capturing the right direction $\left(\phi\left[x_{1}(n)\right] \rightarrow \phi\left[x_{2}(n)\right]\right)$ of connectivity in the feature space $(c=1.00$ and $N=750)$ at confidence level of $\alpha=0.01$.
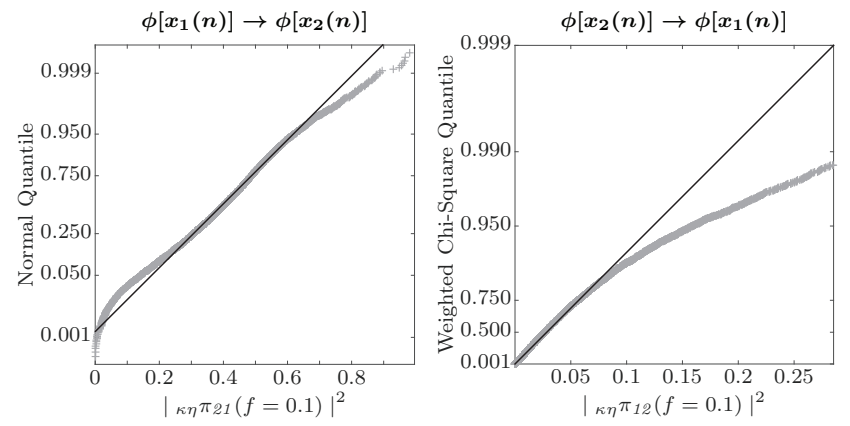

Figure 2: Comparison between the actually observed knPDC with that inferred based on the sample run knPDC of Figure 1. 
Whereas Figure 2 contrasts knPDC ensemble behaviour with that predicted, based on the sample run result of Figure 1 at $f=f_{0}=0.1$, Figure 3 portrays the actual ensemble data distribution against the best fits to a normal distribution $\left(\phi\left[x_{1}(n)\right] \rightarrow \phi\left[x_{2}(n)\right]\right)$ and an $a \chi_{\nu}^{2}$ distribution $(a=0.05, \nu=1.08)$ for $\phi\left[x_{2}(n)\right] \rightarrow \phi\left[x_{1}(n)\right]$. Both Figures 2 and 3 point to a slightly high predicted FP rate at $N=750$ which is confirmed by Table 1 results where one immediately sees the FP convergence to $1 \%$ as $N$ increases.
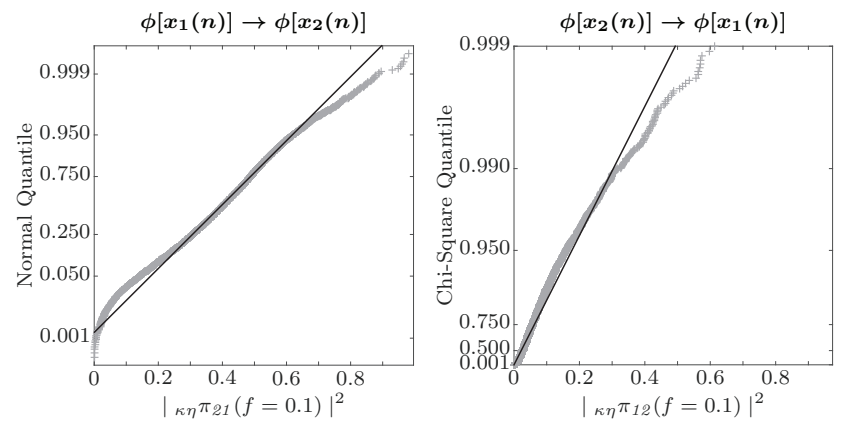

Figure 3: Ensemble asymptotic behaviour of applying the criteria from Baccalá et al. (2013), at $f=f_{0}=$ 0.1 using $\kappa(\mathbf{x}, \mathbf{y})=\left(\mathbf{x}^{\top} \mathbf{y}\right)^{4}(N=750$ and $c=1.00)$, showing the approximate normal behaviour for the existing connection $\phi\left[x_{1}(n)\right] \rightarrow \phi\left[x_{2}(n)\right]$, against the distribution of the non-existing $\phi\left[x_{2}(n)\right] \rightarrow \phi\left[x_{1}(n)\right]$, compared to the expected $a \chi_{\nu}^{2}$ distribution that best fits the data $(a=0.05, \nu=1.08)$.

\begin{tabular}{|c|c|c|c|c|c|c|c|c|c|c|c|}
\hline & & \multicolumn{5}{|c|}{$\mathbf{T P}-100 \times(1-\widehat{\boldsymbol{\beta}})$} & \multicolumn{5}{|c|}{$\mathbf{F P}-\mathbf{1 0 0} \times(\widehat{\alpha})$} \\
\hline Kernel & $c^{N}$ & 100 & 250 & 500 & 750 & 1000 & 100 & 250 & 500 & 750 & 1000 \\
\hline \multirow{3}{*}{$\kappa(\mathbf{x}, \mathbf{y})=\langle\mathbf{x}, \mathbf{y}\rangle^{2}$} & $0 \cdot 10$ & $98 \cdot 18$ & \begin{tabular}{|l|}
99.97 \\
\end{tabular} & $100 \cdot 00$ & $100 \cdot 00$ & $100 \cdot 00$ & 46.50 & 34.92 & 28.57 & 24.98 & $22 \cdot 75$ \\
\hline & 0.50 & 99.98 & $100 \cdot 00$ & $100 \cdot 00$ & $100 \cdot 00$ & $100 \cdot 00$ & $57 \cdot 01$ & $43 \cdot 59$ & 34.56 & $29 \cdot 22$ & $26 \cdot 32$ \\
\hline & $1 \cdot 00$ & $99 \cdot 98$ & $100 \cdot 00$ & $100 \cdot 00$ & $100 \cdot 00$ & $100 \cdot 00$ & $57 \cdot 24$ & $43 \cdot 57$ & 34.55 & $29 \cdot 23$ & $26 \cdot 47$ \\
\hline \multirow{3}{*}{$\kappa(\mathbf{x}, \mathbf{y})=\langle\mathbf{x}, \mathbf{y}\rangle^{4}$} & $0 \cdot 10$ & $72 \cdot 38$ & $90 \cdot 60$ & 96.05 & $97 \cdot 71$ & 99.03 & $34 \cdot 60$ & $15 \cdot 07$ & $5 \cdot 24$ & 1.94 & 0.97 \\
\hline & 0.50 & 82.93 & 94.92 & 97.95 & 98.81 & 98.71 & $43 \cdot 05$ & $19 \cdot 16$ & $6 \cdot 70$ & 2.56 & 1.29 \\
\hline & 1.00 & $83 \cdot 21$ & 95.02 & 97.92 & 98.81 & 98.68 & $43 \cdot 26$ & $19 \cdot 16$ & 6.75 & $2 \cdot 52$ & 1.32 \\
\hline
\end{tabular}

Table 1: Observed knPDC true positive (TP) and false positive (FP) detection rates under quadratic and bi-quadratic homogenous polynomial kernels of orders $d=2$ and 4 , respectively, for the connections $\phi\left[x_{1}(n)\right] \rightarrow \phi\left[x_{2}(n)\right]$ and $\phi\left[x_{2}(n)\right] \rightarrow \phi\left[x_{1}(n)\right]\left(f=f_{0}=0.1\right)$.

\section{Discussion}

After introducing a generalized form of PDC and how to compute the required time series coupling model (8), we used an example to investigate the possibility that connectivity detection criteria developed for treating linear VAR models (Baccalá et al., 2013) could still be nonetheless be useful, even if only as an approximate guideline. Our simulations in the example point to reasonably good performance, approaching the expected $\mathbf{F P}$ rate as $N \rightarrow \infty$.

The choice of (9) was in part dictated by the possibility of making ready comparisons with results in Massaroppe et al. (2011) whose approach was based on preprocessing the data into time varying estimates of the time series approximate entropy (Pincus, 2008) and whose relationships were then investigated using linear models. Whereas that methodology managed to capture quadratic connections, in all counts, the present one outperforms the latter by dispensing with the need for approximate entropy reconstruction parameters whose values proved responsible for the large degrees of sensitivity inherent to the latter method's results.

Our current efforts are geared towards expanding this investigation to systems whose dynamics goes beyond that of the narrow bandwidth represented by (9) and towards rigorously establishing connectivity criteria based for connectivity detection including time domain criteria as in Baccalá et al. (1998), which 
applied Wald type likelihood tests.

An important open issue regards kernel choice. Our preliminary evidence suggests that polyspectral analysis can be useful in this regard (Nikias and Petropulu, 1993).

Finally it is interesting to mention that bivariate causality representations involving nonlinear systems have appeared before (Marinazzo et al., 2014, 2008) though they are more akin to what we called influentiability in Baccalá and Sameshima (2014a,b) rather than to causality, because of the data partialization capability of vector autoregressive modeling.

For the present case, the optimal kernel is the (bi-)quadratic one, due to the conditions of the coupling, as (perhaps) expected. Therefore, this points to studying the present technique specially in cases of models in larger dimensions. Exploratory data analysis of further examples is under way, as is the use of kernels other than homogenous polynomials.

\section{Acknowledgments}

L.M. gratefully acknowledges support from an institutional Ph.D. CAPES Grant. L.A.B. is also gratefully acknowledged to CNPq Grant 307163/2013 - 0 .

\section{References}

Baccalá, L. A., de Brito, C. S. N., Takahashi, D. Y., and Sameshima, K. (2013). Unified asymptotic theory for all partial directed coherence forms. Philos. T. Roy. Soc. A, 371(1997):20120158.

Baccalá, L. A. and Sameshima, K. (2001). Partial directed coherence: A new concept in neural structure determination. Biol. Cybern., 84(6):463-474.

Baccalá, L. A. and Sameshima, K. (2014a). Causality and influentiability: The need for distinct neural connectivity concepts. In Brain Informatics and Health, volume 8609, pages 424-435.

Baccalá, L. A. and Sameshima, K. (2014b). Methods in Brain Connectivity Inference through Multivariate Time Series Analysis, chapter Multivariate time series brain connectivity: A sum up, pages 245-251. CRC Press, Boca Raton.

Baccalá, L. A., Sameshima, K., Ballester, G., do Valle, A. C., and Timo-Iara, C. (1998). Studying the interaction between brain structures via directed coherence and Granger causality. Applied Sig. Process., (5):40-48.

Faes, L., Nollo, G., and Chon, K. H. (2008). Linear and nonlinear parametric model identification to assess Granger causality in short-term cardiovascular interactions. Comput. Cardiol., 35:793-796.

Granger, C. W. J. (1969). Investigating causal relations by econometric models and cross-spectral methods. Econometrica, 37(3):424-438.

He, F., Billings, S. A., Wei, H.-L., and Sarrigiannis, P. G. (2014). A nonlinear causality measure in the frequency domain: Nonlinear partial directed coherence with applications to EEG. J. Neurosci. Meth., (225):71-80.

Hlaváčková-Schindler, K., Paluš, M., Vejmelka, M., and Bhattacharya, J. (2007). Causality detection based on information-theoretic approaches in time series analysis. Phys. Rep., 441(1):1-46.

Kallas, M., Honeine, P., Francis, C., and Amoud, H. (2013). Kernel autoregressive models using YuleWalker equations. Signal Process., 93(11):3053-3061.

Kannan, R. and Tangirala, A. K. (2014). Correntropy-based partial directed coherence for testing multivariate Granger causality in nonlinear processes. Physical Review E, 89(6):062144.

Kumar, R. and Jawahar, C. V. (2007). Kernel approach to autoregressive modeling. In Thirteenth National Conference on Communications (NCC 2007), Kanpur, India. Available in: <http://cvit.iiit.ac.in/papers/ranjeeth07Kernel.pdf>. Accessed in: October/06/2014.

Lütkepohl, H. (2005). New introduction to multiple time series analysis. Springer. 
Marinazzo, D., Liao, W., Pellicoro, M., and Stramaglia, S. (2014). Methods in Brain Connectivity Inference through Multivariate Time Series Analysis, chapter Nonlinear parametric granger causality in dynamical networks, pages 135-159. CRC Press, Boca Raton.

Marinazzo, D., Pellicoro, M., and Stramaglia, S. (2008). Kernel method for nonlinear Granger causality. Phys. Rev. Lett., 100(14):144103.

Massaroppe, L., Baccalá, L. A., and Sameshima, K. (2011). Semiparametric detection of nonlinear causal coupling using partial directed coherence. In Eng. Med. Biol. Soc. Ann., pages 5927-5930.

Nikias, C. and Petropulu, A. P. (1993). Higher order spectra analysis: A non-linear signal processing framework. Prentice Hall.

Paluš, M., Komárek, V., Hrnčíŕ, Z., and Štěrbová, K. (2001). Synchronization as adjustment of information rates: Detection from bivariate time series. Phys. Rev. E, 63(4):046211.

Parzen, E. (1959). Statistical inference on time series by Hilbert space method, I. Technical Report 23, Applied Mathematics and Statistics Laboratory, Stanford University.

Pereda, E., Quiroga, R. Q., and Bhattacharya, J. (2005). Nonlinear multivariate analysis of neurophysiological signals. Prog. Neurobiol., 77(1-2):1-37.

Pincus, S. M. (2008). Approximate entropy as an irregularity measure for financial data. Econometric Reviews, 27(4-6):329-362.

Schelter, B., Winterhalder, M., Eichler, M., Peifer, M., Hellwig, B., Guschlbauer, B., Lücking, C. H., Dahlhaus, R., and Timmer, J. (2006). Testing for directed influences among neural signals using partial directed coherence. J. Neurosci. Meth., 152(1-2):210-219.

Shawe-Taylor, J. and Cristianini, N. (2004). Kernel methods for pattern analysis. Cambridge University Press.

Takahashi, D. Y., Baccalá, L. A., and Sameshima, K. (2010). Information theoretic interpretation of frequency domain connectivity measures. Biol. Cybern., 103(6):463-469. 
A.3 ESTE 2015

\title{
A Simulation Comparison between Fractional Differencing Exponent Estimators
}

\author{
Lucas Massaroppe and Luiz A. Baccalá \\ Universidade de São Paulo \\ lucasmassaroppe@usp.br, baccala@lcs.poli.usp.br
}

\begin{abstract}
Through Monte Carlo simulations we compared the performance of wavelet-based and spectrum-based estimators for the long-memory parameter by computing small sample bias, standard deviations and mean-square errors. The MODWPT (Maximum Overlap Discrete Wavelet Packet Transform) using LA(8) wavelet filter method was shown to outperform all other options under a minimum mean-square error criterion.
\end{abstract}

Keywords: Fractionally integrated models, Long-memory, Wavelets, Whittle likelihood.

\section{Introduction}

Over the past decade, estimation of long-memory parameter $(d)$ in fractionally integrated processes has become important for many data driven fields like astronomy (Percival and Walden, 2000), hydrology (Hurst, 1951), econometrics (Gençay et al., 2002) and internet traffic (Veitch and Abry, 1999). The most popular methods (a) use log-periodogram regression or (b) maximize the local Whittle likelihood, though the latter demands numerical maximization to obtain $\widehat{d}$ (Phillips and Shimotsu, 2004; Shimotsu and Phillips, 2005). An alternative proposed in Jensen (1999); Whitcher (2004) involves wavelet-based semiparametric log-scale regression. Here we investigate and compare the latter estimators.

The rest of this paper is organized as follows: Section 2 briefly review the concepts of fractionally integrated processes. In Section 3 we summarize the estimation methods, which is followed by brief discussion (Section 4) and results (Section 5) with conclusions in Section 6. 


\section{Fractionally Integrated Processes}

A process $\{x(n)\}_{n \in \mathbb{Z}}$ is fractionally integrated with long memory parameter $d$ if

$$
(1-L)^{d} x(n)=w(n),
$$

holds, where $\{w(n)\}_{n \in \mathbb{Z}} \sim$ i.i.d.N $\left(0, \sigma_{w}^{2}\right)$ for not necessarily integer valued $d$.

Equation (1) may be rewritten with help of the binomial expansion

$$
\sum_{j=0}^{n} \frac{(-d)_{j}}{j !} x(n-j)=w(n),
$$

where

$$
(d)_{j}=\frac{\Gamma(d+j)}{\Gamma(d)}=d(d+1) \cdots(d+j-1)
$$

is Pochhammer's symbol, for the forward factorial function and $\Gamma(\cdot)$, the gamma function. Note that $(1-L) x(n)=x(n)-x(n-1)$ is an integrated process of order one (denoted as $I(1)$ ). Therefore, (1) is a generalization of integrated processes.

Consequently, one may obtain $\{x(n)\}_{n \in \mathbb{Z}}$ by inverting (1),

$$
x(n)=(1-L)^{-d} w(n)=\sum_{j=0}^{n-1} \frac{(d)_{j}}{j !} w(n-j),
$$

which holds for all $d$.

\section{Fractional Parameter Estimation Methods}

The methods we consider are the approximate local Whittle method (LW) (Phillips and Shimotsu, 2004) including its variant - the exact local Whittle estimator (ELW) (Shimotsu and Phillips, 2005) which we contrast to the maximal overlap discrete wavelet packet transform (MODWPT)-based method, put forward by Whitcher (2004) through our own implementation (Massaroppe et al., 2014).

From Geweke and Porter-Hudak (1983); Jensen (1999); Phillips and Shimotsu (2004); Shimotsu and Phillips (2005); Whitcher (2004), the above estimators obey a log-linear relationships in their computed magnitudes against the independent variable of interest whose slope allows computing $d$. Also the latter estimators have been shown to be consistent as well as asymptotically normal (Jensen, 1999; Phillips and Shimotsu, 2004; Shimotsu and Phillips, 2005).

\section{Simulations}

To compare method performance, long-memory processes were obtained for $d$ in $\{0.1,0.2,0.3,0.4,0.75,1\}$ and realization lengths $N$ in $\{128,256,512,1024,2048\}$, using (4). Prior to analysis, a burn-in period of 10,000 points was used to avoid 
transient phenomena. Long-memory process simulations followed the guidelines in Jensen and Nielsen (2014). The choice of realization lengths as powers of 2 prevented dyadic wavelet computation boundary effects and dispensed with zeropadding (Jensen, 1999). Estimator performance was computed based on using 10,000 Monte Carlo replicates.

Full result disclosure is available for download at

$$
\text { http://www.lcs.poli.usp.br/ baccala/longmemorywavepaper2.zip. }
$$

Good statistical properties for $d$ were ensured by following the recommendations in Gençay et al. (2002); Gneiting et al. (2012); Jensen (1999); Percival and Walden (2000); Phillips and Shimotsu (2004); Shimotsu and Phillips (2005); Whitcher and Jensen (2000). In the case of the Whittle estimators, $m=\left\lfloor N^{0.65}\right\rfloor$ Fourier frequencies were used in the computations whereas for the MODWPT approach, two different wavelet filter classes were examined: the Extremal Phase (Haar and D(4)) and Least Asymmetric (LA(8)) filters.

\section{Results}

Simulation results are analogous to those in Massaroppe et al. (2014) for both spectral and wavelet methods. The related $d$-value is most often underestimated. As $d$ increases and $N$ remains fixed, there is no consistent pattern for the bias in the wavelet-based case (Figure 3). Under the same conditions, bias almost always decreases for the local Whittle case. When it comes to the standard deviation (SD), it decreases regardless of estimator type (Figures 1, 2 and 3 shows the average mean, bias, standard-deviation (SD) and mean-square error (MSE) against length $\left.\left(\log _{2} N\right)\right)$.

The mean square error (MSE) showed a similar decrease pattern for all methods as $N$ grows. Moreover, for all methods MSE values were insensitive to changes in $d$, for fixed $N$ (Figure 4).

The box-plots of Figure 5 sum up the present comparison for $N=512$ data points.

\section{Conclusion and Comments}

This work enlightened some of the differences between consistent fractional differencing estimators. Consistency was confirmed (Section 5). It was possible to observe that the $\mathbf{L A}(8)$ wavelet-based method was consistently better in least mean-square error terms whilst providing acceptable bias. This is especially interesting given the tendency of frequency domain methods to misbehave above $d=0.5$ (Shimotsu and Phillips, 2005), something that is easy to appreciate in Figure 5. 

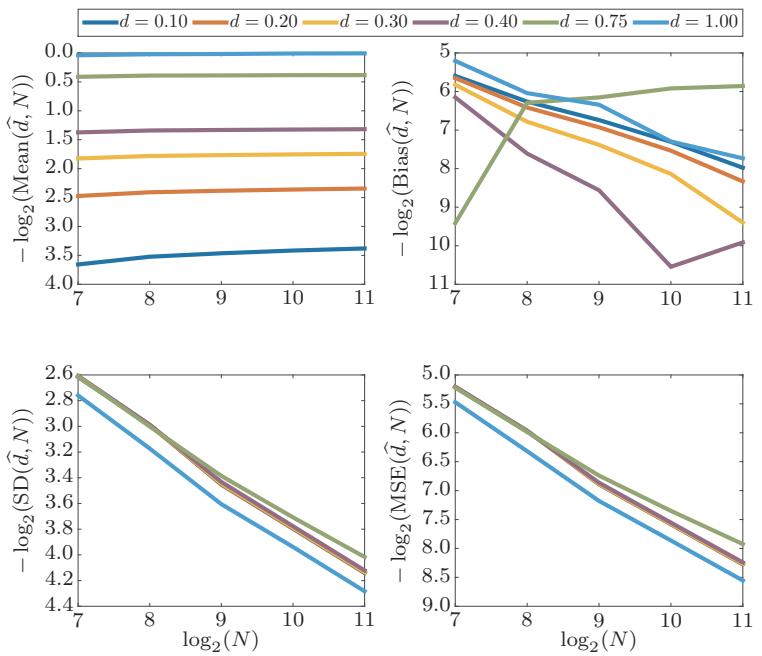

Figure 1: Average Monte-Carlo results for the approximate local Whittle maximum likelihood estimator.
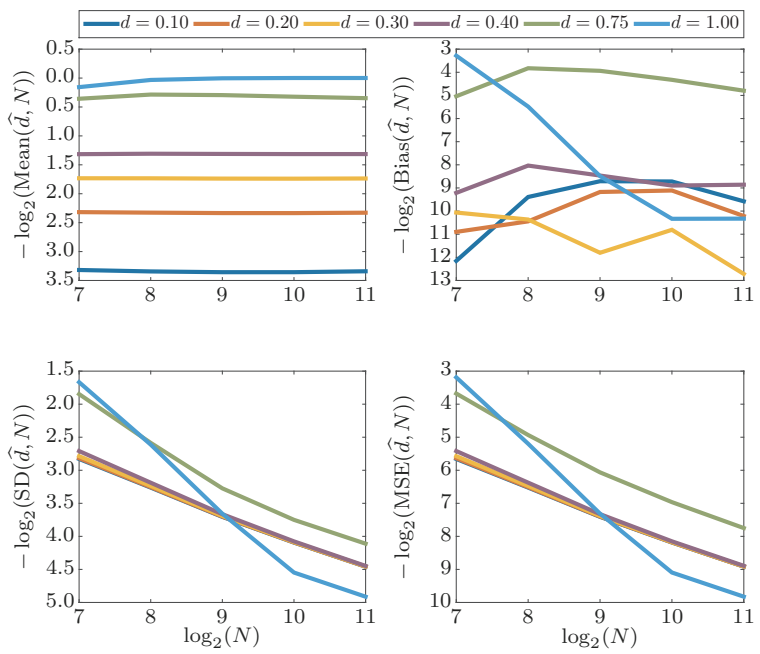

Figure 2: Average Monte-Carlo results for the exact local Whittle maximum likelihood estimator. 

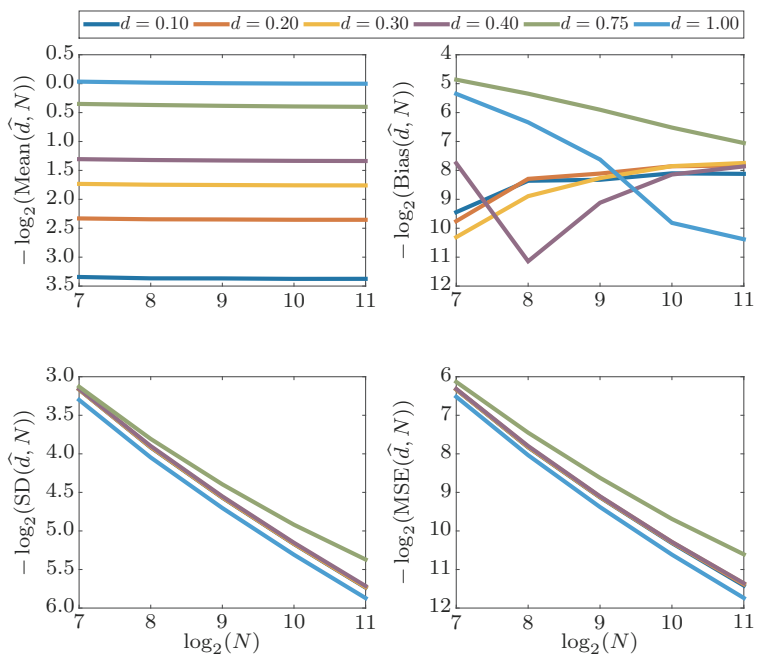

Figure 3: Average Monte-Carlo results for the wavelet-based estimator, using LA(8) filter.

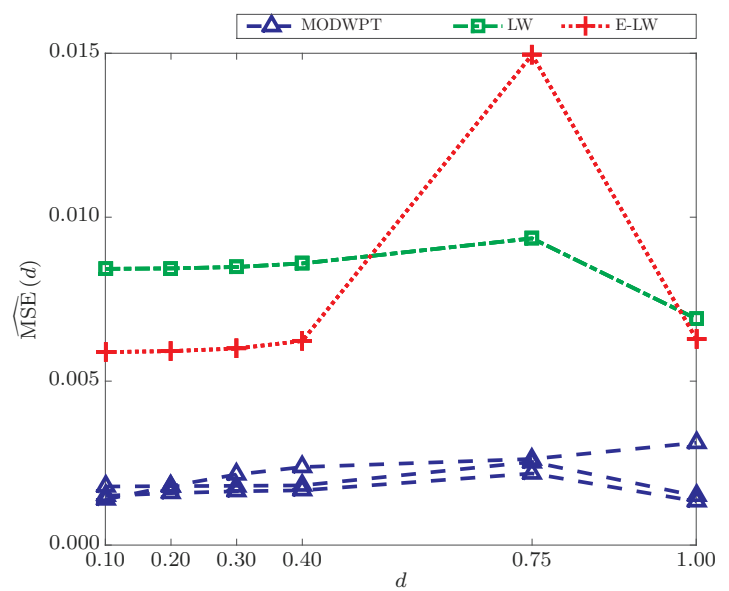

Figure 4: Mean-squared error as function of $d(N=512)$. For the MODWPT-based estimators, the following holds $\widehat{\operatorname{MSE}}(d)_{\mathbf{L A}(8)}<\widehat{\operatorname{MSE}}(d)_{\mathbf{D}(4)}<\widehat{\operatorname{MSE}}(d)_{\mathbf{H a a r}}$. 


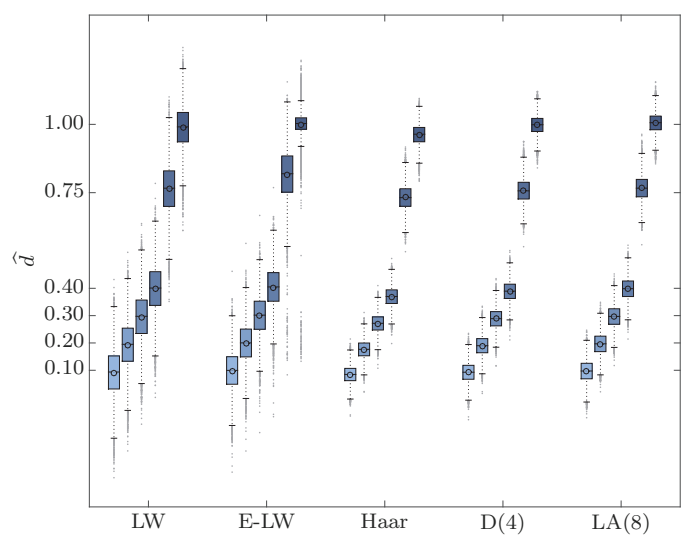

Figure 5: The MODWPT-based and periodogram-based methods, with $N=512$ data points. Comparison between different filters, Haar, $\mathbf{D}(4), \mathbf{L A}(8)$, used to estimate $d$, the approximate $(\mathbf{L W})$ and the exact $(\mathbf{E}-\mathbf{L W})$ local Whittle maximum likelihood estimators for $d$.

\section{Acknowledgments}

L.M. gratefully acknowledges support from an institutional Ph.D. CAPES Grant. L.A.B. also gratefully acknowledges CNPq Grant 307163/2013 - 0.

\section{References}

Gençay, R., Selçuk, F., and Whitcher, B. (2002). An introduction to wavelets and other filtering methods in finance and economics. Academic Press, San Diego, California.

Geweke, J. and Porter-Hudak, S. (1983). The estimation and application of long memory time series models. Journal of Time Series Analysis, 4(4):221-238.

Gneiting, T., Ševčíková, H., and Percival, D. B. (2012). Estimators of fractal dimension: Assessing the roughness of time series and spatial data. Statistical Science, $27(2): 247-277$.

Hurst, H. E. (1951). Long term storage capacity of reservoirs. Transactions of the American Society of Civil Engineers, 116:770-799.

Jensen, A. N. and Nielsen, M. Ø. (2014). A fast fractional difference algorithm. Working Papers 1307, Queen's University, Department of Economics. 
Jensen, M. J. (1999). Using wavelets to obtain a consistent ordinary least squares estimator of the long memory parameter. Journal of Forecasting, 18:17-32.

Massaroppe, L., Caduda, F., and Baccalá, L. A. (2014). On the performance of wavelet-based long-memory parameter estimation. Natal, RN. Associação Brasileira de Estatística (ABE). Available in: <http://www.ime.usp.br/ abe/sinape2014/trabalhos/anais\#187>. Accessed in: March/16/2015.

Percival, D. B. and Walden, A. T. (2000). Wavelet methods for time series analysis. Cambridge Series in Statistical and Probabilistic Mathematics. Cambridge University Press, Cambridge.

Phillips, P. C. B. and Shimotsu, K. (2004). Local Whittle estimation in nonstationary and unit root cases. The Annals of Statistics, 32(2):656-692.

Shimotsu, K. and Phillips, P. C. B. (2005). Exact local Whittle estimation of fractional integration. The Annals of Statistics, 33(4):1890-1933.

Veitch, D. and Abry, P. (1999). A wavelet-based joint estimator of the parameters of long-range dependence. IEEE Transactions on Information Theory, 45(3):878897.

Whitcher, B. (2004). Wavelet-based estimation for seasonal long-memory processes. Technometrics, 46(2):225-238.

Whitcher, B. and Jensen, M. J. (2000). Wavelet estimation of a local long memory parameter. Exploration Geophysics, 31(2):94-103. 


\title{
A.4 EMBC 2015
}

\section{Kernel-nonlinear-PDC extends Partial Directed Coherence to Detecting Nonlinear Causal Coupling}

\author{
Lucas Massaroppe and Luiz A. Baccalá
}

\begin{abstract}
Here we investigate a new concept, kernelnonlinear-Partial Directed Coherence, whereby a kernel feature nonlinear-Part detecting nonlinear causal links that are otherwise undetectable through linear modeling. We show that adequate connectivity detection is achievable by applying asymptotic decision criteria similar to the ones developed for linear models.

Keywords: Nonlinear-Granger causality; Kernel-NonlinearPartial Directed Coherence; Inference
\end{abstract}

\section{INTRODUCTION}

Because of its ability to expose potential data-driven causal explanations for dynamic observations the subject of causality between time series has seen increased interest over the last decade or so [1]-[9] specially in neuroscience applications, because of its potential in precluding invasive procedures.

The extensive review [10] examines many estimation methods geared towards exposing the connectivity between neural regions via time series observations, e.g., from EEG and fMRI. One popular means towards that goal is via Granger Causality (GC) [11] estimation, due to its ability to provide a measure of information flow between series [12], [13]

Most currently practical measures are linear in nature, such as directed transfer function (DTF) [1] and partial directed coherence (PDC) [3] among them which have repeatedly proven to be robust estimators of connectivity in the frequency domain, due to well known properties of linear vector autoregressive (VAR) models that include fast convergence and known asymptotics [14]. These techniques have even been used with some success for nonlinear systems [15], yet it is possible to show they fail to capture connectivity when coupling is mediated by even powered polynomial contributions [16].

Several proposals have been put forward to deal with this shortcoming [17]-[22]. In common they share convergence problems by often needing a large number of data points to fit a large number of descriptive parameters or require optimization of non convex functionals. Another issue is the lack of rigorously defined statistical connectivity criteria as opposed to the linear case [14], [23].

To overcome the latter hindrances, we investigate a recently proposed method, termed kernel-nonlinear-Partial Directed Coherence [24] that applies PDC to properly 'kernelized' data.

The authors are with Escola Politécnica, Department of Telecommunications and Control Engineering, University of São Paulo, Brazil, 05508 - 900. lucasmassaroppe@usp.br, baccala@lcs.poli.usp.br
Here we use kernels other than the Gaussian (adopted by [24]) and estimate a multivariate kernel vector autoregressive model via implicit kernel mapping [25], [26]. Through the 'kernelized' covariance structure, we compute asymptotic statistics, by analogy with [23], in the feature space and show that this correctly allows inferring the direction of interaction between channels.

The rest of this paper is organized as follows: Section II briefly recaps kernel nonlinear partial directed coherence (knPDC) [3] and how it is estimated. In Section III we illustrate method performance using the chaotic map (10), under the application of the asymptotic frequency domain criteria from [23]. A brief discussion with conclusions appears in Section IV.

\section{THE METHOD}

To capture general couplings, we represent the input series $\left\{x_{i}(n)\right\}_{n=1}^{N}$ (input space) through a Kernel Vector Autoregressive (kVAR) model, such as

$$
\phi[\mathbf{x}(n)]=\sum_{r=1}^{p} \mathbf{A}^{\phi}(r) \phi[\mathbf{x}(n-r)]+\boldsymbol{\vartheta}^{\phi}(n),
$$

with $\left\{\boldsymbol{\vartheta}^{\phi}(n)\right\}_{n \in \mathbb{Z}}$ i.i.d. zero mean and covariance matrix $\Sigma_{\vartheta^{\phi}}$.

The $\phi(\cdot)$ function leading to the kernel generated feature space represents a nonlinear mapping [27] $(\phi: \mathcal{X} \rightarrow \mathbf{F})$, such that

$\mathbb{E}\left\{\phi\left[x_{i}(n)\right] \phi\left[x_{i}(n-k)\right]\right\}=\mathbb{E}\left\{\kappa\left[x_{i}(n), x_{i}(n-k)\right]\right\}$,

where $\kappa(\cdot)$ is a Mercer kernel.

\section{A. Kernel-nonlinear-Partial Directed Coherence}

Model (1) allows defining of kernel-nonlinear-PDC (kn$P D C)$ as

$$
{ }_{\kappa \eta} \pi_{i j}(f)=\frac{\bar{A}_{i j}^{\phi}(f) / \sqrt{\sigma_{i i}^{\phi}}}{\sqrt{\overline{\mathbf{a}}_{j}^{\phi \mathrm{H}}(f) \boldsymbol{\Sigma}_{\vartheta^{\phi}}^{-1} \overline{\mathbf{a}}_{j}^{\phi}(f)}},
$$

with

$$
\bar{A}_{i j}^{\phi}(f)=\delta_{i j}-\sum_{l=1}^{p} a_{i j}^{\phi}(l) \mathrm{e}^{-\mathbf{i} 2 \pi f l}, \quad\left(\mathbf{i}^{2}=-1\right),
$$

where $a_{i j}^{\phi}(l)$ are the coefficients of an adequately fit kVAR model, while $\overline{\mathbf{a}}_{j}^{\phi}(f)$ represent the columns of the $\left[\bar{A}_{i j}^{\phi}(f)\right]$ matrix. 
To fit (1) we generalize the approach in [25], [26], to the multivariate case so that

$$
\boldsymbol{\Phi}_{i}=\mathbf{A}^{\phi}\left(\mathbf{X} \mathbf{J}_{i}\right)^{\top}+\boldsymbol{\Theta}_{i}^{\phi},
$$

where

$$
\begin{array}{rlr}
\boldsymbol{\Phi}_{i} & =(\phi[\mathbf{x}(1)], \cdots, \phi[\mathbf{x}(N)]) & (K \times N), \\
\mathbf{A}^{\phi} & =\left(\mathbf{A}^{\phi}(1), \cdots, \mathbf{A}^{\phi}(p)\right) & (K \times K p), \\
\mathbf{X}(n) & =\left[\begin{array}{c}
\phi[\mathbf{x}(n)] \\
\vdots \\
\phi[\mathbf{x}(n-p+1)]
\end{array}\right] & (K p \times 1), \\
\mathbf{J}_{i} & =\left[\begin{array}{c}
\mathbf{0}_{(i-K p-1) \times K p} \\
\mathbf{I}_{K p} \\
\mathbf{0}_{(N-i+1) \times K p}
\end{array}\right],
\end{array}
$$
Kernel Condition (2). The necessary long-memory (LM) parameter for each series was obtained via [32]'s procedure as implemented in [33].

Figure 1 depicts $i$ PDC (information PDC) [12] based on linear modeling for a typical input space input realization ( $c=1.0$ and $N=1024$ ) after fractional differencing, under the asymptotic detection criteria (at $\alpha=1 \%$ ) from [23] showing its inability to capture the existing $x_{1}(n) \rightarrow x_{2}(n)$ coupling.

that through the application of the vec-operator

$$
\begin{aligned}
\boldsymbol{\varphi}_{i}=\operatorname{vec}\left(\boldsymbol{\Phi}_{i}\right) & (K N \times 1), \\
\boldsymbol{\alpha}^{\phi}=\operatorname{vec}\left(\mathbf{A}^{\phi}\right) & \left(K^{2} p \times 1\right), \\
\boldsymbol{\theta}_{i}^{\phi}=\operatorname{vec}\left(\boldsymbol{\Theta}_{i}^{\phi}\right) & (K N \times 1),
\end{aligned}
$$

gives

$$
\boldsymbol{\varphi}_{i}=\left(\mathbf{X} \mathbf{J}_{i} \otimes \mathbf{I}_{K}\right) \boldsymbol{\alpha}^{\phi}+\boldsymbol{\theta}_{i}^{\phi} .
$$

Now, the least squares estimate for $\boldsymbol{\alpha}^{\phi}$ is given by

$$
\begin{aligned}
\widehat{\boldsymbol{\alpha}}^{\boldsymbol{\phi}} & =\left(\sum_{i=p+1}^{N} \mathbf{J}_{i}^{\top} \mathbf{X}^{\top} \mathbf{X} \mathbf{J}_{i}\right)^{-1}\left(\sum_{i=p+1}^{N} \mathbf{J}_{i}^{\top} \mathbf{X}^{\top} \boldsymbol{\varphi}_{i}\right) \\
& =\left(\sum_{i=p+1}^{N} \mathbf{J}_{i}^{\top} \mathbf{K} \mathbf{J}_{i}\right)^{-1}\left(\sum_{i=p+1}^{N} \mathbf{J}_{i}^{\top} \mathbf{K}^{i}\right)
\end{aligned}
$$

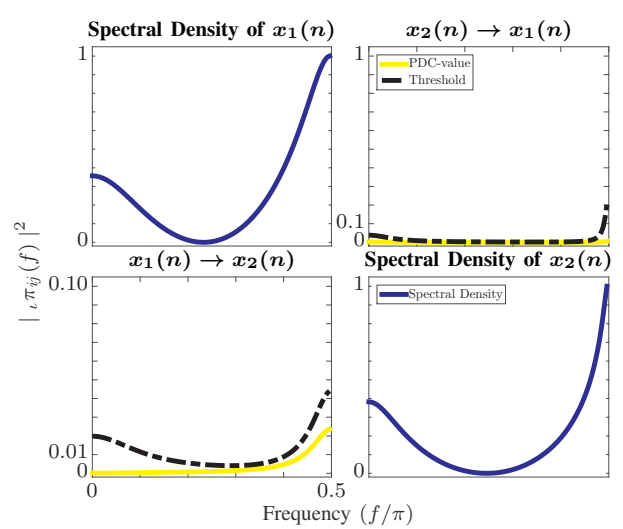

(9) Fig. 1: $i$ PDC for a sample run of (10) incapable of capturing the connection in the right direction $\left(x_{1}(n) \rightarrow x_{2}(n)\right)$ using input connection in the right direction $\left(x_{1}(n) \rightarrow x_{2}(n)\right)$ using input
space data $(c=1.0$ and $N=1024)$ at confidence level of $\alpha=$ space data $(c=1.0$ and $N=1024)$ at confidence level of $\alpha=$
0.01 . The graphs along the main diagonal portray the series logspectra in arbitrary units.

Figure 2 shows knPDC, for $\kappa(\mathbf{x}, \mathbf{y})=\langle\mathbf{x}, \mathbf{y}\rangle+|\langle\mathbf{x}, \mathbf{y}\rangle|^{8}$, using the same data as Figure 1, but assuming that the asymptotic criteria in [23] also hold for feature space descriptions. It is immediately apparent that correct connectivity detection is attained as for the $\operatorname{knPDC}$ for $\phi\left[x_{1}(n)\right] \rightarrow \phi\left[x_{2}(n)\right]$ is above the dashed threshold line. Connection absence in the opposite direction is also confirmed for knPDC is below threshold.
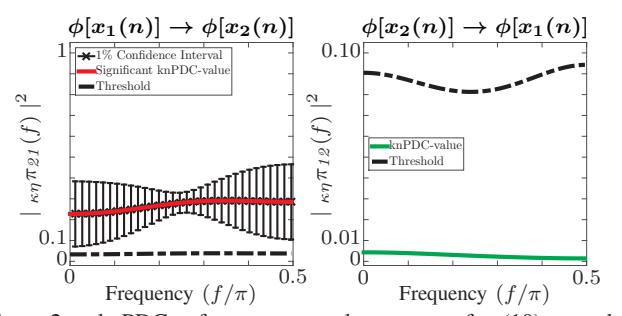

Fig. 2: knPDC for a sample run of (10), under $\kappa(\mathbf{x}, \mathbf{y})=\langle\mathbf{x}, \mathbf{y}\rangle+|\langle\mathbf{x}, \mathbf{y}\rangle|^{8}$, capable of capturing the right direction $\left(\phi\left[x_{1}(n)\right] \rightarrow \phi\left[x_{2}(n)\right]\right)$ of connectivity in the feature space $(c=1.0$ and $N=1024)$ at the confidence level of $\alpha=0.01$.

Table I shows further details about the procedure's de- 
tection capabilities as a function of $c$ and $N$ using 10,000 Monte Carlo replicates. Additional ensemble behaviour can be appreciated in Figure $3(c=1.0)$ showing a good fit to the distributions one would infer from [23] based on the data of Figure 2. Reasonably good fit to a normal for the existing connection is observed and up to the $99 \%$ percentile the non existing connection approaches the weighted sum of $\chi_{1}^{2}$ predicted by [23]. In the latter case, large tails only happen above $99 \%$. Fit improves with $N$ as can be seen from Figures 3 and 4 .

\begin{tabular}{c|c|c|c||c|c|c|} 
& \multicolumn{2}{|c|}{$\mathbf{2}-\mathbf{1 0 0} \times(\mathbf{1}-\widehat{\boldsymbol{\beta}})$} & \multicolumn{3}{c|}{$\mathbf{F P}-\mathbf{1 0 0} \times(\widehat{\boldsymbol{\alpha}})$} \\
\hline \multicolumn{1}{c|}{} & $\mathbf{2 5 6}$ & $\mathbf{5 1 2}$ & $\mathbf{1 0 2 4}$ & $\mathbf{2 5 6}$ & $\mathbf{5 1 2}$ & $\mathbf{1 0 2 4}$ \\
\hline 0.5 & 23.80 & 40.52 & $72 \cdot 17$ & 0.28 & 0.00 & 0.00 \\
1.0 & 58.54 & 88.40 & 99.56 & 0.51 & 0.04 & 0.00 \\
$2 \cdot 0$ & 46.77 & 82.13 & 98.86 & 0.45 & 0.00 & 0.00 \\
\hline
\end{tabular}

TABLE I: Observed knPDC true positive (TP) and false positive (FP) detection rates under the kernel $\kappa(\mathbf{x}, \mathbf{y})=\langle\mathbf{x}, \mathbf{y}\rangle+|\langle\mathbf{x}, \mathbf{y}\rangle|^{8}$, respectively, for the connections $\phi\left[x_{1}(n)\right] \rightarrow \phi\left[x_{2}(n)\right]$ and $\phi\left[x_{2}(n)\right] \rightarrow \phi\left[x_{1}(n)\right]$.

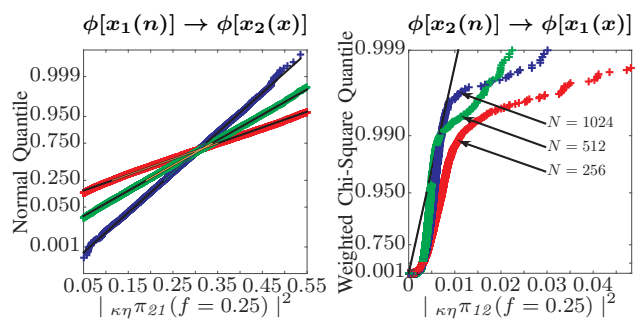

Fig. 3: Comparison between the actually observed knPDC with that inferred based on the sample run knPDC of Figure $2(c=1.0)$. Note the distribution convergence as $N$ increases.

This scenario is confirmed in Figure 4 showing the distributions against their best fits respectively to a normal for $\phi\left[x_{1}(n)\right] \rightarrow \phi\left[x_{2}(n)\right]$ and to an $a \chi_{\nu}^{2}$ distribution $\left(a=4.3 \times 10^{-4}, \nu=7.4\right)$ for $\phi\left[x_{2}(n)\right] \rightarrow \phi\left[x_{1}(n)\right]$ that approximates the weighted sum of $\chi_{1}^{2}$ distributions from [23]. Both scenarios are confirmed by Table I results.

\section{DISCUSSION AND FUTURE WORK}

Here we have empirically shown a kernelized technique for estimating nonlinear connectivity in cases where ordinary linear techniques fail. The present results extend those presented in [34] for a band limited oscillator that is quadratically coupled to another system which achieves superior performance than the method discussed in [16] where we proposed a technique based on windowed sample entropy flow measurements.

Whereas investigation to rigorously justify the observed behaviour is under way, heuristically it is possible to argue that kernelization unwraps the frequency domain mappings that are induced by nonlinear connectivity terms thereby linearizing the problem into an asymptotic behaviour like that
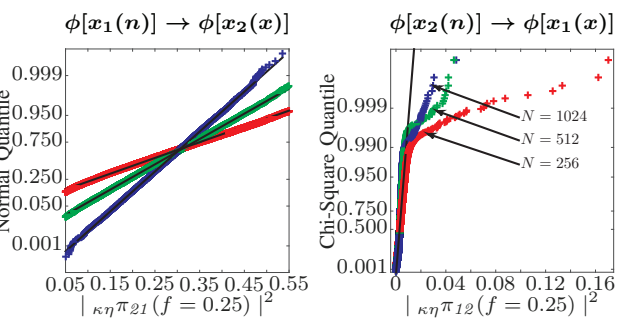

Fig. 4: Ensemble asymptotic behaviour of applying the criteria from [23], at $f=0.25$ using $\kappa(\mathbf{x}, \mathbf{y})=\langle\mathbf{x}, \mathbf{y}\rangle+|\langle\mathbf{x}, \mathbf{y}\rangle|^{8}(N=1024$ and $c=1.0$ ), showing the approximate normal behaviour for the existing connection $\phi\left[x_{1}(n)\right] \rightarrow \phi\left[x_{2}(n)\right]$, against the distribution of the non-existing $\phi\left[x_{2}(n)\right] \rightarrow \phi\left[x_{1}(n)\right]$, compared to the expected of the non-existing $\phi\left[x_{2}(n)\right] \rightarrow \phi\left[x_{1}(n)\right]$, compared to the expected
$a \chi_{\nu}^{2}$ distribution that best fits the data $\left(a=4.3 \times 10^{-4}, \nu=7.4\right.$, $a \chi_{\nu}^{2}$ distribution
$N=1024)$

of the linear case. This unwrapping ensures that frequencies that are mapped outband by the nonlinearity and hence elude linear capture, once again have the opportunity of residing in the same frequency bands of the feature space series thereby enabling their capture. Consequently, it is possible that one can produce nonlinear kernel Wald type Granger Causality tests analogous to those in [14] and a kernel nonlinear Directed Transfer Function as a natural generalization of linear DTF, both of which are in progress with promising preliminary results.

So far, our investigations suggest that a judicious kernel choice is crucial and should be done to appropriately cover the existing nonlinearities and whose determination may be done with help of standard polyspectral techniques [35].

Finally note that the present results are not immediately comparable to those in [29], [30], even though the latter also employs a kernel approach. This impossibility stems from the fractional difference pre-processing of the data whose use proved essential in the present case.

\section{ACKNOWLEDGMENT}

L.M. gratefully acknowledges support from an institutional Ph.D. CAPES Grant. L.A.B. is also gratefully acknowledges to CNPq Grant 307163/2013 - 0 .

\section{References}

[1] M. J. Kamiński and K. J. Cieslak-Blinowska, "A new method of the description of the information flow in the brain structures," Biological Cybernetics, vol. 65, pp. 203-210, 1991.

2] L. A. Baccalá, K. Sameshima, G. Ballester, A. C. do Valle, and C. Timo-Iara, "Studying the interaction between brain structures via directed coherence and Granger causality," Applied Signal Processing, no. 5 , pp. $40-48,1998$.

[3] L. A. Baccalá and K. Sameshima, "Partial directed coherence: A new concept in neural structure determination," Biological Cybernetics, vol. 84 , no. 6 , pp. 463-474, 2001

[4] M. Ding, S. L. Bressler, W. Yang, and H. Liang, "Short-window spectral analysis of cortical event-related potentials by adaptive multispectral analysis of cortical event-related potentials by adaptive multiand variability assessment," Biological Cybernetics, vol. 83, no. 1, pp. and 45,2000 - 
[5] L. Astolfi, F. Cincotti, D. Mattia, M. G. Marciani, L. A. Baccalá, F. de Vico Fallani, S. Salinari, M. Ursino, M. Zavaglia, L. Ding, J. C. Edgar, G. A. Miller, B. He, and F. Babiloni, "Comparison of different cortical connectivity estimators for high-resolution EEG recordings," Human Brain Mapping, vol. 28, no. 2, pp. 143-157, 2007.

[6] L. Astolfi, F. Cincotti, D. Mattia, M. G. Marciani, L. A. Baccalá, F. de Vico Fallani, S. Salinari, M. Ursino, M. Zavaglia, and F. Babiloni, "Assessing cortical functional connectivity by partial directed coherence: Simulations and application to real data" IEEE Transactions on Biomedical Engineering, vol. 53, no. 9, pp. 1802-1812, 2006.

[7] K. J. Cieslak-Blinowska, R. Kuś, M. J. Kamiński, and J. JędrzejewskaK. J. Cieslak-Blinowska, R. Kus, M. J. Kaminski, and J. Jędrzejewska-
Szmek, "Transmission of brain activity during cognitive tasks," Brain Szmek, "Transmission of brain activity duin
Topography, vol. 23, pp. 205-213, 2010.

[8] J. R. Sato, D. Y. Takahashi, S. M. Arcuri, K. Sameshima, P. A. Morettin, and L. A. Baccalá, "Frequency domain connectivity identification: An application of partial directed coherence in fMRI," Human Brain Mapping, vol. 30, no. 2, pp. 452-461, 2009

[9] A. Fujita, P. Severino, J. R. Sato, and S. Miyano, "Granger causality in systems biology: Modeling gene networks in time series microarray data using vector autoregressive models," in Advances in Bioinformatics and Computational Biology, ser. Lecture Notes in Computer Science, C. Ferreira, S. Miyano, and P. Stadler, Eds. Springer Berlin / Heidelberg, 2010, vol. 6268, pp. 13-24.

[10] P. A. Valdes-Sosa, A. Roebroeck, J. Daunizeau, and K. Friston, "Effective connectivity: Influence, causality and biophysical modeling," Neurolmage, vol. 58, no. 2, pp. 339-361, September 2011.

[11] C. W. J. Granger, "Investigating causal relations by econometric models and cross-spectral methods," Econometrica, vol. 37, no. 3, pp. $424-438,1969$.

[12] D. Y. Takahashi, L. A. Baccalá, and K. Sameshima, "Information theoretic interpretation of frequency domain connectivity measures," Biological Cybernetics, vol. 103, no. 6, pp. 463-469, 2010.

[13] — - "Frequency domain connectivity: An information theoretic perspectives," in 2010 Annual International Conference of the IEEE Engineering in Medicine and Biology Society $(E M B C)$, Buenos Aires, September 2010, pp. 1726-1729.

[14] H. Lütkepohl, New introduction to multiple time series analysis. Berlin: Springer, 2005

[15] B. Schelter, M. Winterhalder, M. Eichler, M. Peifer, B. Hellwig, B. Guschlbauer, C. H. Lücking, R. Dahlhaus, and J. Timmer, "Testing B. Guschlbauer, C. H. Lücking, R. Dahlhaus, and J. Timmer, "Testing
for directed influences among neural signals using partial directed for directed influences among neural signals using partial directed
coherence," Journal of Neuroscience Methods, vol. 152, no. 1-2, pp. coherence," Journa

[16] L. Massaroppe, L. A. Baccalá, and K. Sameshima, "Semiparametric detection of nonlinear causal coupling using partial directed coherence," in 2011 Annual International Conference of the IEEE Engineering in Medicine and Biology Society, EMBC, Boston, August 30 2011-Sepember 32011 2011, pp. 5927-5930.

[17] K. Hlaváčková-Schindler, M. Paluš, M. Vejmelka, and J. Bhattacharya, "Causality detection based on information-theoretic approaches in time series analysis," Physics Reports - Review Section of Physics Letters, vol. 441, no. 1, pp. 1-46, March 2007

[18] D. Marinazzo, M. Pellicoro, and S. Stramaglia, "Kernel method for nonlinear Granger causality," Physical Review Letters, vol. 100, no. 14, p. 144103, April 2008 .

[19] M. Paluš, V. Komárek, Z. Hrnčîr, and K. Štěrbová, "Synchronization as adjustment of information rates: Detection from bivariate time series," Physical Review E, vol. 63, no. 4, p. 046211, March 2001.

[20] L. Faes, G. Nollo, and K. H. Chon, "Linear and nonlinear parametric model identification to assess Granger causality in short-term cardiovascular interactions," Computers in Cardiology, vol. 35, pp. 793-796, 2008 .

[21] E. Pereda, R. Q. Quiroga, and J. Bhattacharya, "Nonlinear multivariate analysis of neurophysiological signals," Progress in Neurobiology, vol. 77 , no. 1-2, pp. 1-37, 2005.

[22] F. He, S. A. Billings, H.-L. Wei, and P. G. Sarrigiannis, "A nonlinear causality measure in the frequency domain: Nonlinear partial directed coherence with applications to EEG," Journal of Neuroscience Methods, no. 225, pp. 71-80, 2014

[23] L. A. Baccalá, C. S. N. de Brito, D. Y. Takahashi, and K. Sameshima, "Unified asymptotic theory for all partial directed coherence forms," Philosophical Transactions of the Royal Society A: Mathematical, Physical and Engineering Sciences, vol. 371, no. 1997, p. 20120158, July 2013.
[24] R. Kannan and A. K. Tangirala, "Correntropy-based partial directed coherence for testing multivariate Granger causality in nonlinear processes," Physical Review E, vol. 89, no. 6, p. 062144, June 2014.

[25] R. Kumar and C.V. Jawahar, "Kernel approach to autoregressive modeling," in Thirteenth National Conference on Communications (NCC 2007). Kanpur, India: Indian Institute of Technology, Kanpur, January 2007, available in: <http://cvit.iiit.ac.in/papers/ranjeeth07Kernel.pdf>. Accessed in: October/06/2014

[26] M. Kallas, P. Honeine, C. Francis, and H. Amoud, "Kernel autoregressive models using Yule-Walker equations," Signal Processing, vol. 93, no. 11, pp. 3053-3061, 2013

[27] E. Parzen, "Statistical inference on time series by Hilbert space method, I," Applied Mathematics and Statistics Laboratory, Stanford University, Stanford, Tech. Rep. 23, January 1959.

[28] J. Shawe-Taylor and N. Cristianini, Kernel methods for pattern analysis. Cambridge University Press, 2004.

[29] Y. Chen, G. Rangarajan, J. Feng, and M. Ding, "Analyzing multiple Y. Chen, G. Rangarajan, J. Feng, and M. Ding, "Analyzing multiple
nonlinear time series with extended Granger causality," Physics Letters nonlinear time series with extended Grange

[30] I. Park and J. C. Príncipe, "Correntropy based Granger causality," in IEEE International Conference on Acoustics, Speech and Signal Processing, 2008. ICASSP 2008., April 2008, pp. 3605-3608.

[31] A. W. Lo, "Long-term memory in stock market prices," Econometrica, vol. 59, no. 5, pp. 1279-1313, September 1991.

[32] B. Whitcher, "Wavelet-based estimation for seasonal long-memory processes," Technometrics, vol. 46, no. 2, pp. 225-238, 2004.

[33] L. Massaroppe, F. Caduda, and L. A. Baccalá, "On the performance of wavelet-based long-memory parameter estimation." Natal, RN: Associação Brasileira de Estatística (ABE), July 2014, available in: <http://www.ime.usp.br/ abe/sinape2014/trabalhos/anais\#187>. Accessed in: March/16/2015.

[34] L. Massaroppe and L. A. Baccalá, "Detecting nonlinear Granger causality via the kernelization of partial directed coherence." Rio de Janeiro, RJ: ISI - International Statistical Institute, July 2015, accepted.

[35] C. Nikias and A. P. Petropulu, Higher order spectra analysis: A non-linear signal processing framework, ser. Prentice Hall Signal Processing Series. Prentice Hall, April 1993. 


\title{
A.5 EMBC 2016
}

\section{Causal Connectivity via Kernel Methods: Advances and Challenges}

\author{
Lucas Massaroppe and Luiz A. Baccalá
}

\begin{abstract}
Here we argue that kernel methods applied to common linear connectivity determination methods are able to reveal the presence of nonlinear causal links between time series via asymptotic decision criteria similar to the ones developed for multivariate linear time series connectivity modeling.
\end{abstract}

Keywords: Nonlinear-Granger causality; Kernel-NonlinearPartial Directed Coherence; Inference

\section{INTRODUCTION}

Following an initial large proliferation in time series based methods for appraising neural connectivity, rigorous asymptotic results have appeared in connection to linear models (see [1] and [2] for a comparison between some proposed methods). Building on the work of [3], [4] and our own asymptotic results for connectivity detection using partial directed coherence (PDC) [1], we recently showed that it is also possible to reliably detect nonlinear directional interactions through a kernelized version of partial directed coherence [5], [6] $(\kappa \eta P D C)$ whose theoretical basis can be shown to follow from Hable's results [7] regarding asymptotic gaussianity resulting from kernelization.

We were able to show that both canonical multivariate Wald-type time domain Granger causality tests and Directed Transfer Function (DTF) [8] can also be generalized to their kernel versions: $\kappa \eta G C T$ and $\kappa \eta D T F$ respectively, see details in [9].

Borrowing from a structure similar to that from Model 7 in [10], under mutually uncorrelated $N(0,1)$ innovations, kernel methods asymptotically approach correct connectivity detection within the prescribed confidence levels [9]. A glimpse of this result is contained in Table I where predictably quadratic kernels outperform fourth power polynomial ones.

TABLE I: Detection percent rates for existing $1 \rightarrow 2$ and absent $2 \rightarrow 1$ connections under quadratic $\left(K_{1}\right)$ and fourth degree $\left(K_{2}\right)$ polynomial kernels using 1024 data points and 10,000 Monte Carlo replications

$\begin{array}{ccccc}\text { Method } & 1 \rightarrow 2\left(K_{1}\right) & 1 \rightarrow 2\left(K_{2}\right) & 2 \rightarrow 1\left(K_{1}\right) & 2 \rightarrow 1\left(K_{2}\right) \\ \kappa \eta G C T & 1.0 & 3.3 & 96.6 & 81.8 \\ \kappa \eta P D C & 1.1 & 4.4 & 96.7 & 89.0 \\ \kappa \eta D T F & 1.37 & 3.4 & 96.3 & 80.0\end{array}$

\section{DISCUSSION}

Our results show that kernelization can rigorously help expose nonlinear intereactions, yet our extensive simulations

The authors are with Escola Politécnica, Department of Telecommunications and Control Engineering, University of São Paulo, Brazil, 05508 - 900. lucasmassaroppe@usp.br, baccala@lcs.poli.usp.br display some patterns: (a) kernel choice is critical (as seen on Table I), (b) data outliers make the use of robust feature space estimation algorithms mandatory, which is a complicating practical issue; (c) connection detectability diminishes as models increase in order and in the number of simultaneously processed signals.

These observations imply a long road ahead before this methodology can be made practical as practical kernel model goodness-of-fit associated with proper kernel choice need to be systematically developed. On the theoretical side one must develop proper quantitative and qualitative interpretation of the results since quantifiers like $\kappa \eta P D C / \kappa \eta D T F$ essentially amount to forms of feature space spectral decompositions.

\section{ACKNOWLEDGMENT}

L.M. gratefully acknowledges support from an institutional Ph.D. CAPES Grant. L.A.B. is also gratefully acknowledges to CNPq Grant 307163/2013 - 0 .

\section{References}

[1] L. A. Baccalá, C. S. N. de Brito, D. Y. Takahashi, and K. Sameshima, "Unified asymptotic theory for all partial directed coherence forms," Philosophical Transactions of the Royal Society A: Mathematical, Physical and Engineering Sciences, vol. 371, no. 1997, p. 20120158, July 2013.

[2] K. Sameshima, D. Y. Takahashi, and L. A. Baccalá, "On the statistical performance of Granger-causal connectivity estimators" Brain Informatics, April of

[3] I. Park and J. C. Príncipe, "Correntropy based Granger causality," in IEEE International Conference on Acoustics, Speech and Signal in IEEE International Conference on Acoustics, Speech and
Processing, 2008. ICASSP 2008., April 2008, pp. 3605-3608.

[4] D. Marinazzo, M. Pellicoro, and S. Stramaglia, "Kernel method for D. Marinazzo, M. Pellicoro, and S. Stramaglia, "Kernel method for
nonlinear Granger causality," Physical Review Letters, vol. 100, no. 14, nonlinear Granger caus

[5] L. Massaroppe and L. A. Baccalá, "Detecting nonlinear Granger causality via the kernelization of partial directed coherence," in Proceedings of the 60th World Statistics Congress of the International Statistical Institute, ISI2015. Rio de Janeiro, RJ: International Statistical Institute, The Hague, The Netherlands, July 2015, pp. 2036204

[6] - "Kernel-nonlinear-PDC extends Partial Directed Coherence to detecting nonlinear causal coupling," in 2015 37th Annual International Conference of the IEEE Engineering in Medicine and Biology Society (EMBC), Milan, August 2015, pp. 2864-2867.

[7] R. Hable, "Asymptotic normality of support vector machine variants and other regularized kernel methods," Journal of Multivariate Analysis, vol. 106, pp. 92-117, 2012.

[8] M. J. Kamiński and K. J. Cieslak-Blinowska, "A new method of the description of the information flow in the brain structures," Biological Cybernetics, vol. 65, pp. 203-210, 1991.

[9] L. Massaroppe, "Estimação da causalidade de Granger no caso de interação não-linear," Tese de Doutorado, Escola Politécnica da Universidade de São Paulo, São Paulo, Agosto 2016.

[10] B. Gourévitch, R. L. Bouquin-Jeannès, and G. Faucon, "Linear and nonlinear causality between signals: Methods, examples and neurophysiological applications," Biological Cybernetics, vol. 95, no. 4, pp. 349-369, 2006 . 